\title{
Targeting and Modulation of the Tumor Stroma in Pancreatic Cancer
}


Targeting and Modulation of the Tumor Stroma in Pancreatic Cancer JONAS SCHNITTERT 


\section{Graduation Committee}

$\begin{array}{lll}\text { Chairman } & \text { Prof. Dr. ir. J.W.M. Hilgenkamp } & \text { University of Twente } \\ \text { Supervisor } & \text { Prof. Dr. G. Storm } & \text { University of Twente } \\ \text { Co-supervisor } & \text { Dr. J. Prakash } & \text { University of Twente } \\ \text { Members } & \text { Prof. Dr. P. Jonkheijm } & \text { University of Twente } \\ & \text { Prof. Dr. M. van de Laar } & \text { University of Twente } \\ & \text { Prof. Dr. J. Klaase } & \text { University Medical Center Groningen } \\ & \text { Prof. Dr. R. Brock } & \text { Radboud University Medical Center } \\ & \text { Prof. Dr. Y. van Kooyk } & \text { VU University Medical Center }\end{array}$

The studies in this thesis have been carried out at the University of Twente, Department of Biomaterials Science and Technology between 2015 and 2018.

\section{UNIVERSITY OF TWENTE.}

The research was supported by the University of Twente and the Swedish Research Council (project no. 2011-5389).

\section{Y Vetenskapsrådet UNIVERSITY OF TWENTE.}

The printing of this thesis was financially supported by the Faculty of Science and Technology, University of Twente, The Netherlands.

Title: Targeting and Modulation of the Tumor Stroma in Pancreatic Cancer

Author: Jonas Schnittert

ISBN: 978-90-365-4585-3

DOI: $10.3990 / 1.9789036545853$

(C) 2018 Jonas Schnittert. All right reserved.

Cover design by Jonas Schnittert

Printed by Ipskamp printing 


\title{
TARGETING AND MODULATION OF THE TUMOR STROMA IN PANCREATIC CANCER
}

\author{
DISSERTATION
}

to obtain

the degree of doctor at the University of Twente,

on the authority of the rector magnificus,

prof.dr. T.T.M. Palstra,

on account of the decision of the graduation committee,

to be publicly defended

on Friday the $6^{\text {th }}$ of July 2018 at 12.45 hours

by

\section{JONAS SCHNITTERT}

Born on the $10^{\text {th }}$ of July 1989

in Solingen, Germany 
This thesis has been approved by:

Supervisor: Prof. Dr. G. Storm, University of Twente

Co-supervisor: Dr. J. Prakash, University of Twente 
Paranymphs: Marcel Alexander Heinrich

Dr. Praneeth Reddy Kuninty 

Dedicated to my family, friends and colleagues 



\section{TABLE OF CONTENTS}

CHAPTER 1

General Introduction, Aim and Outline

CHAPTER 2

MicroRNA targeting to modulate tumor microenvironment

(Published in Frontiers in Oncology, 2016)

CHAPTER 3

Anti-microRNA targeting using peptide- based nanocomplexes to inhibit

differentiation of human pancreatic stellate cells

(Published in Nanomedicine, 2017)

CHAPTER 4

Reprogramming tumor stroma using an endogenous lipid lipoxin A4 to treat pancreatic cancer

(Published in Cancer Letters, 2018)

CHAPTER 5

111

Integrins in wound healing, fibrosis and tumor stroma: High potential targets for therapeutics and drug delivery

(Published in Advanced Drug Delivery Reviews, 2018)

CHAPTER 6

169

Integrin $\alpha 11$ regulates differentiation of pancreatic stellate cells in pancreatic cancer

CHAPTER 7

199

A novel peptide against integrin alpha11 to target nanoparticles to cancerassociated fibroblasts

CHAPTER 8

Summary and Discussion

\section{APPENDIX}

A: Nederlandse Samenvatting

B: List of Publications and Scientific Awards

C: Acknowledgements 



\section{Chapter 1- General Introduction}

\subsection{Pancreatic ductal adenocarcinoma}

The first description of pancreatic cancer was provided by Giovanni Battista Morgagni in $1761^{1}$. The most common form of pancreatic cancer is pancreatic ductal adenocarcinoma (PDAC) ${ }^{2}$. Until today, PDAC has one of the poorest prognosis of all cancers with the lowest 5-year-relative survival rate $(8 \%)$, and presents an unsolved problem in cancer medicine ${ }^{3}$. Each year, around 100,000 people in Europe and 50,000 people in the USA get diagnosed with PDAC ${ }^{4}$. According to the American Cancer Society PDAC belongs, only in females, to the top 10 of estimated new cases for 2018, with 26,240 estimated cases in the USA. Strikingly, PDAC is ranked fourth for the number of estimated death in 2018 in males (behind lung \& bronchus, prostate and colon \& rectum) and females (behind lung \& bronchus, breast and colon \& rectum), with 23,020 and 21,310 new cases, respectively. However, PDAC is expected to become the second cause of cancer-related death by $2020^{5}$. Of the five cancers with the highest number of estimated death in males and females, identified by the American Cancer Society, PDAC has the worst prognosis in The Netherlands regarding the survival rate (Figure 1).

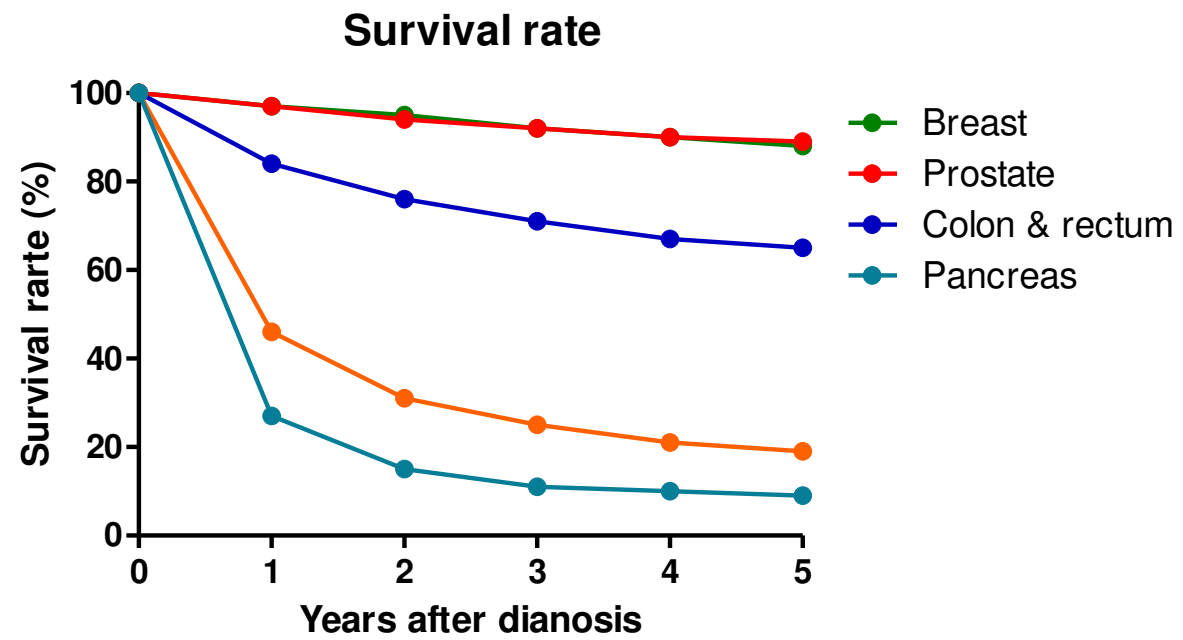

Figure 1. Survival rate in percent for prostate, breast, colon \& rectum, lung and pancreatic cancer in The Netherlands. Pancreatic cancer shows the poorest survival rate in the Netherlands for the period from $2011-2015^{6}$. 


\subsection{Diagnosis and risk factors}

The risk to develop PDAC over a lifetime is $1.49 \%{ }^{7}$. Most PDAC incidences occur between 60 and 80 years of age ${ }^{5}$. The symptoms of PDAC become stronger with the development of the disease and include mid-epigastric pain, weight loss, malaise, nausea and fatigue ${ }^{5}$.

The diagnosis of PDAC is mostly dependent on the symptoms of the disease, which only appear late during disease progression. Therefore, around $50 \%$ of patients with PDAC present with locally advanced or metastatic disease at the time of diagnosis, with the main metastatic sites including the liver, lungs and peritoneum ${ }^{7}$. Therefore, most PDACs are diagnosed when there is no longer any chance for cure. Early diagnosis might be possible in patients with an increased risk to develop PDAC or a family history of PDAC ${ }^{5}$. It is estimated that around $90 \%$ of all PDAC cases are sporadic while the other $10 \%$ are hereditary ${ }^{5}$. The main environmental risk factors for sporadic PDAC are tobacco smoking, obesity, long standing diabetes mellitus and alcohol induced pancreatitis ${ }^{8-11}$. For hereditary PDAC patients might present genetic alterations. Mutations known to be associated with PDAC include STK11 mutation recognized in Peutz-Jeghers syndrome, the cationic trypsinogen gene PRSS1 and SPINK1 mutations associated with hereditary pancreatitis, the p16 mutation linked to familial atypical multiple mole melanoma (FAMMM), the adenomatous polyposis coli (APC) mutation responsible for familial adenomatous polypois (FAP), BRCA1 and BRCA2 mutations increasing the risk for hereditary breast ovary cancer (HBOC), MLH1 and MSH2 gene mutations associated with hereditary nonpolyposis colon cancer (HNPCC), and the ataxia telangiectasia (ATM) mutation ${ }^{5}$.

Generally, the examination of pancreatic cancer is performed by computer tomography (CT) and in uncertain cases magnet resonance imaging (MRI) and endoscopic ultrasound (EUS) may be used to image ductal structures ${ }^{5}$. In accordance with the International Cancer of Pancreas (CAPS) Consortium, patients presenting genetic risk factors or a family history of pancreatic cancer should be initially screened with EUS or MRI/magnetic resonance cholangiopancreatography ${ }^{12}$. However, more cost effective and practical screening methods like blood biomarkers are required for PDAC. The Sialyl Lewis carbohydrate antigen (CA 19-9) is the only clinical serum biomarker for PDAC with a sensitivity and specificity of around $80 \%$. However, the sensitivity is lowered in early PDAC and therefore CA 19-9 is not applicable for screening and currently only used for therapeutic monitoring ${ }^{13,14}$. The search for novel 
biomarkers for PDAC is an intense field of research. The detection of circulating tumor cells ${ }^{15-17}$ or circulating tumor DNA ${ }^{18,19}$ from liquid biopsies have the potential to be effective tools in the prognosis of pancreatic cancer.

\subsection{Standard treatment of PDAC}

Whenever possible PDAC patients are treated with a surgical resection of the tumor. The preoperative evaluation of PDAC patients is based on the assessment of the relationship between the tumor and the major adjacent vessels, portal vein $(\mathrm{PV})$, superior mesenteric vein (SMV), superior mesenteric artery (SMA), celiac axis (CA), hepatic artery (HA), and the presence of metastasis ${ }^{5}$. The respectability of PCAD is dependent on the absence of distant metastasis, the lack of SMV or PV distortion and clear fat planes around the CA, HA and SMA ${ }^{20}$. Additionally, the consideration for pancreatic resection involves a risk-benefit assessment ${ }^{2}$.

The majority of PDAC cases are metastatic at the time of diagnosis and a surgical resection of these tumors is not feasible. Therefore, patients with metastatic PDAC are mostly treated with chemotherapy with the aim to reduce symptoms and prolong life. Fluorouracil (5-FU) has been the main standard of care but was replaced by the superior gemcitabine ${ }^{21}$. Next, a combination therapy of gemcitabine together with the EGFR inhibitor, erlotinib, resulted in a significant increase in survival time from 5.9 to 6.2 month but added toxicity ${ }^{22}$. In a randomized clinical trial, FOLFIRINOX was compared to gemcitabine and increased survival from 6.8 to 11.1 month ${ }^{23}$. Additionally, survival benefit compared to gemcitabine was achieved by the combination of gemcitabine with nab-paclitaxel (Abraxane), improving the survival time from 6.7 to 8.5 month ${ }^{24}$. Both FOLFIRINOX and Abraxane showed toxic side effects compared to gemcitabine ${ }^{23,24}$ but are currently golden standard treatments for PDAC in patients able to tolerate the side effects.

\subsection{Pathophysiology}

Anatomically, the pancreas is divided into the exocrine pancreas composed of acinar cells, centroacinar cells and ductal cells, responsible for food absorption and the endocrine pancreas, consisting of the Islets of Langerhans that secrete the hormones insulin and glucagon for glucose and lipid homeostasis. With 85$90 \%$ PDAC is the most common form of pancreatic cancer ${ }^{25,26}$. Within invasive PDAC, common genetic mutations include mutational activation of the oncogene KRAS and inactivation the three tumor suppressor genes: TP53, 
p16/CDKNA2A, Smad4 and BRCA2 27,28. Additional genetic abnormalities in invasive PDAC include widespread chromosomal losses, gene amplifications and telomere shortening which is believed to contribute to chromosomal instability ${ }^{26}$.

PDAC develops from histologically different precursor lesions known as pancreatic intraepithelial neoplasia (PanIN), intraductal papillary mucinous neoplasm (IPMN) and mucinous cystic neoplasm (MCN), with decreasing frequency of development, respectively ${ }^{29}$. The majority of invasive PDAC develops from PanIN lesions (Figure 2) ${ }^{26}$. These lesions are characterized by their degree of dysplasia, into PanIN-1, PanIN-2 and PanIN-3 (Figure 2) ${ }^{30}$. Early detection of PanIN lesions present the opportunity to cure pancreatic cancer before the development of an invasive carcinoma ${ }^{27}$. Genetic analysis of PanIN lesions has shown, that PanIN-1 lesions already show mutations of KRAS, p16/CDKN2A and $B R A F^{31,32}$. These findings indicate the earliest genetic events driving pancreatic neoplasia ${ }^{27}$. Next to PanIN, IPMN lesions are precursors of invasive PDAC ${ }^{21}$. Early detection of IPMN lesions could also present the opportunity to cure pancreatic cancer before the development of an invasive carcinoma. Within $95 \%$ of these lesions, genetic mutations of KRAS and/or GNAS were found ${ }^{33}$.

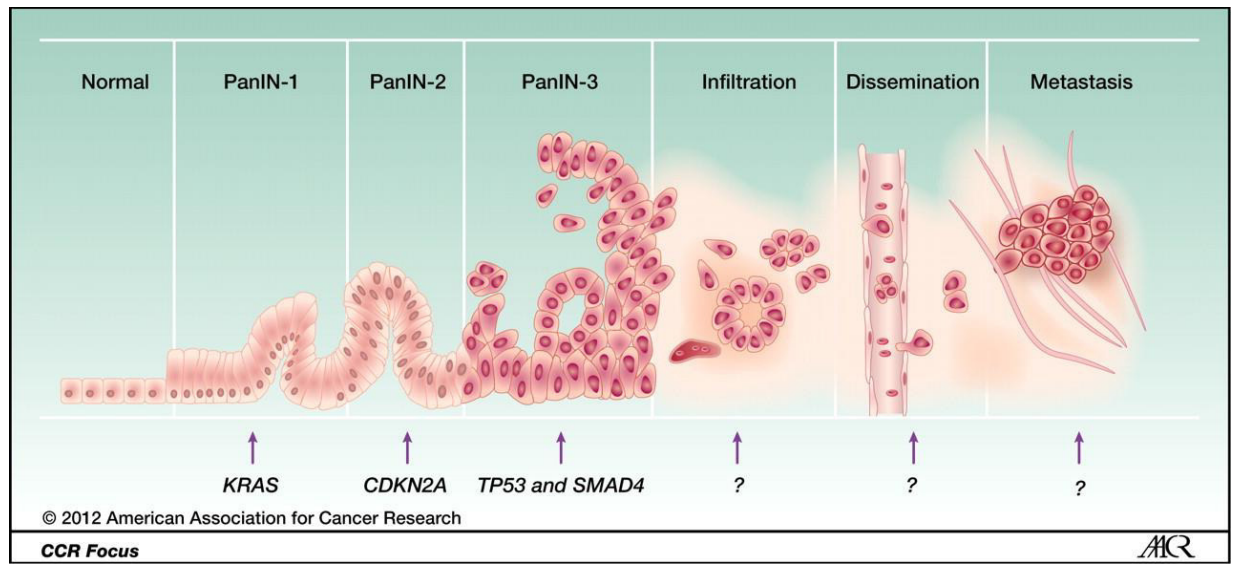

Figure 2. Graphical model for the progression of PDAC from a normal cell to metastatic PDAC, indicating genetic mutation associated with PanIN precursor lesions. The figure was adapted from Iacobuzio-Donahue et al. ${ }^{27}$. 


\subsection{The pancreatic tumor stroma}

PDAC presents with a highly abundant desmoplastic reaction which is also known as the pancreatic tumor microenvironment (TME) or pancreatic tumor stroma. The pancreatic tumor stroma can occupy up to $90 \%$ of the entire tumor volume ${ }^{34}$. The tumor stroma is composed of non-cancerous cells, including cancer-associated fibroblasts (CAFs), tumor-associated macrophages (TAMs), pericytes, endothelial cells, and infiltrating immune cells (e.g. T-cells, natural killer (NK) cells and macrophages), ECM proteins (e.g. collagen and fibronectin) and soluble factors, including cytokines and growth factors ${ }^{35}$. CAFs have been recognized as the most prominent cell type in the tumor stroma of pancreatic cancer ${ }^{36-38}$. Recently, the cellular components, and especially CAFs of the stroma were identified as drivers of tumorigenesis and their role in promoting tumor growth, angiogenesis, invasion and metastasis ${ }^{35,37}$.
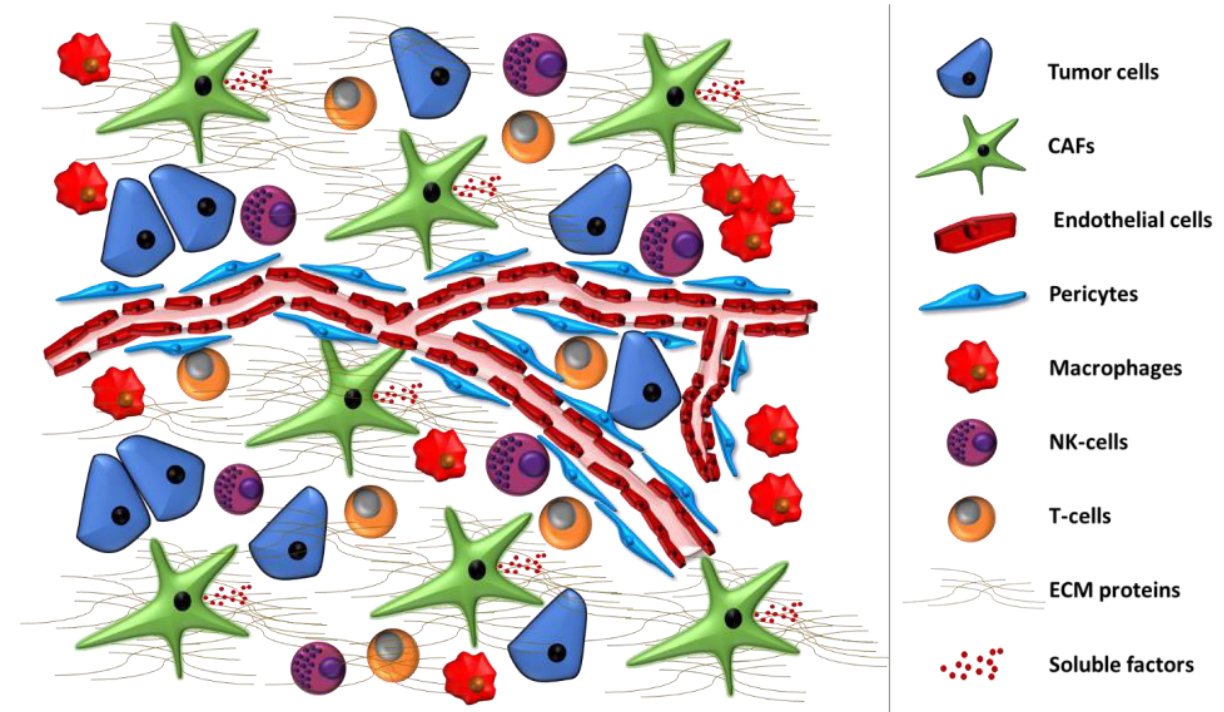

Figure 3. A graphical depiction of the dense tumor stroma in pancreatic cancer. The depiction includes the most prominent cell types, CAFs, endothelial cells, pericytes, macrophages, NK-cells, T-cells, and the non-cellular component, ECM proteins (e.g. collagen, fibronectin) and soluble factors (growth factors, cytokines).

Pancreatic stellate cells which have first been described in 1982 by Watari et al. ${ }^{39}$, have more recently been recognized as precursors of CAFs and their key role in producing the desmoplastic reaction in PDAC ${ }^{40-43}$. Recently, Öhlund et al. have made a first step in defining two different CAF populations by introducing 
myofibroblastic and inflammatory CAFs as CAFs with a distinct function and location in the stroma of PDAC ${ }^{44}$.

\subsection{Pancreatic stellate cells}

Pancreatic stellate cells (PSCs) are star shaped stromal cells, located at the basolateral aspect of acinar cells or surround perivascular and periductal regions in the healthy pancreas, and were first isolated and cultured in $1998^{45,46}$. The functions of quiescent hPSCs include the storage of lipid droplets rich in vitamin A, basic exocrine and endocrine secretion, phagocytosis and immunity ${ }^{47}$. During the development of PDAC, quiescent PSCs become activated via various mechanisms, including risk factors (ethanol and its metabolites, chronic inflammation and smoking), environmental stress (e.g. hypoperfusion, hypoxia, oxidative stress), cellular factors (e.g. IL-1, IL-6, HIF1 $\alpha$, TGF- $\beta$, CCN2), and molecular regulating pathways (e.g. Wnt/ $\beta$-catenin signaling, PI3K pathway), resulting in a transformation of quiescent PSCs into cells with a activated myofibroblast-like phenotype ${ }^{48}$. Once activated, PSCs lose their cytoplasmic vitamin A storing lipid droplets and start to express CAF markers such as $\alpha$ smooth muscle actin which serves as a marker for the identification of activated hPSCs and is also known as a negative prognostic factor in PDAC ${ }^{48,49}$. Furthermore, activated hPSCs are the most important precursors of CAFs ${ }^{48}$. When hPSCs are in the activated state, they acquire a proliferative phenotype and induce desmoplasia by synthesizing abundant extracellular matrix (ECM) components such as collagens, fibronectin, laminin and hyaluronic acid and unbalanced expression of MMPs and TIMPs (Figure 4) ${ }^{43,48}$. Additionally, activated PSCs have an increased secretion of cytokines such as interleukin-1, 6, 8 and 10 (IL-1, 6, 8 and 10) and growth factors, including insulin-like growth factor 1 (IGF1), vascular endothelial growth factor (VEGF), and platelet derived growth factor (PDGF) (Figure 4) ${ }^{43,50,51}$. These growth factors have the ability to promote angiogenesis and proliferation and migration of epithelial cancer cells, invasion and metastasis (Figure 4) 43,48,52. Moreover, cancer cells secrete cytokines as IL-1, 6 and TNF- $\alpha$, and growth factors including TGF- $\beta 1$, PDGF $^{53}$. These interactions result in an active crosstalk between cancer cells and activated hPSCs (Figure 4). 


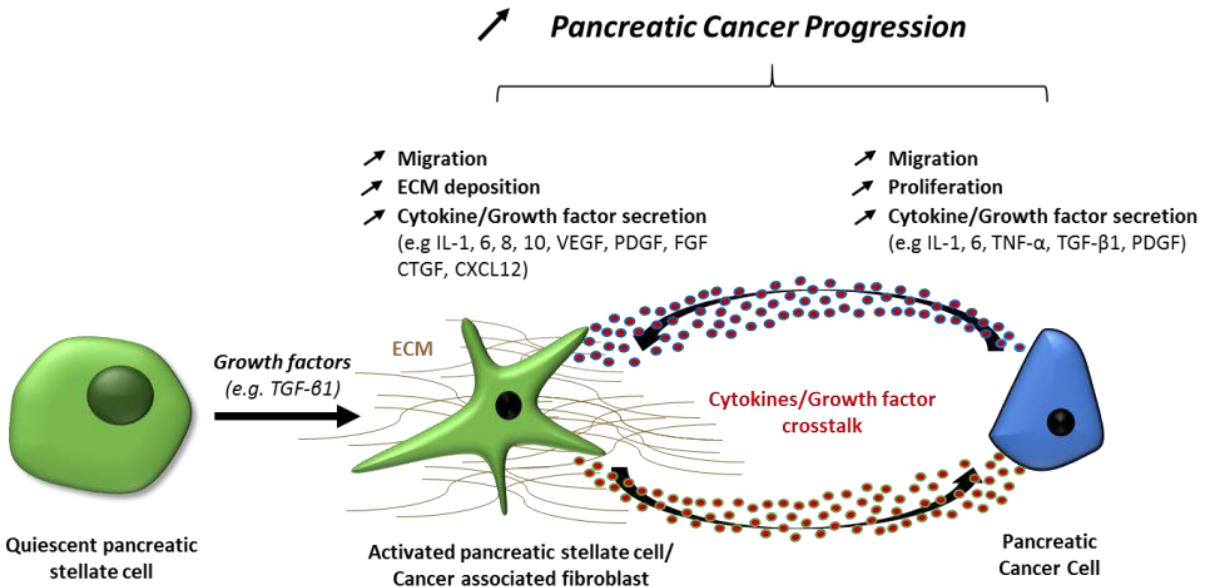

Figure 4. Schematic depiction showing hPSC activation and the interaction between hPSCs and pancreatic cancer cells, contributing to cancer progression.

\subsection{Role of hPSCs in tumor progression}

Within the tumor stroma growth factors, chemokines, cytokines, miRNAs and exosomes secreted by PSCs are known for their ability to act in an autocrine fashion, resulting in PSC activation or exert paracrine signals on epithelial tumor cells to increase the proliferation, migration, and invasion of tumor cells ${ }^{54-58}$. Additionally, paracrine factors secreted by PSCs protect tumor cells from apoptosis, radiotherapy, and chemotherapy ${ }^{59,60}$. Bachem et al. have demonstrated in an in vivo study that the increased ECM deposition in pancreatic cancers results from the paracrine stimulation of PSCs by cancer cells ${ }^{50}$. Another in vivo study showed that when PSCs and Panc-1 epithelial tumor cells are subcutaneously injected in a 1:1 mix in mice, these tumors grow significantly faster and are richer in $\alpha$-SMA and collagen than subcutaneous tumors consisting solely of Panc-1 cells ${ }^{61}$. Very recently, autophagy of pancreatic stellate cells induced by environmental stress and cell-stroma interactions, was reported to be associated with histological grading, peritoneal dissemination, perivascular invasion and lymph node metastasis ${ }^{62}$.

\subsection{Tumor stroma contributes to drug resistance and presents a barrier for therapeutics}

PDAC has a high resistance to chemotherapeutics. There are multiple reasons for this chemo resistance including: (i) reduced drug uptake, (ii) increased energy-dependent drug efflux and (iii) cellular alteration that effect drug 
cytotoxicity such as reduced apoptosis and a dysregulated drug metabolism ${ }^{63}$. For example, a novel molecular mechanism has been proposed for TGF- $\beta$ activated PSCs which express cysteine-rich angiogenic inducer61 (CYR61), a matricellular protein regulating the nucleoside transporters hENT1 and hCNT3 responsible for the cellular uptake of gemcitabine ${ }^{64}$.

Another cause of PDAC chemo resistance involving PSCs is the extensive PSCinduced desmoplastic reaction in PDAC, resulting in intra-tumoral hypoxia and a self-perpetuating fibrosis cycle ${ }^{63}$. Activated PSCs additionally contribute to the chemoresistance via the hypoxia-fibrosis cycle ${ }^{63}$. Thereby, drug delivery to cancer cells is impaired while the epithelial to mesenchymal transition (EMT) and genetic instability of these cells is induced ${ }^{65-67}$.

In general, stroma-rich tumors such as pancreatic cancer are characterized by an abundant amount of ECM proteins, produced to a high extent by PSCs, which act as a physical barrier and therefore prevent drug penetration through blood vessel constriction ${ }^{42,68-70}$. Within PDAC tumor, solid stress has been closely related to drug resistance in PDAC and therapeutic strategies decreasing solid stress show potential therapeutic benefit ${ }^{71,72}$. ECM components such as collagen and hyaluronic acid and PSCs are the main components of the stroma causing solid stress ${ }^{48}$. A few studies investigating the effect of the stroma and/or its' components on drug penetration have been performed. Inhibiting hedgehog signaling to deplete tumor stromal tissue could enhance the delivery of chemotherapy in PDAC tumor-bearing mice ${ }^{68}$. Other studies have enzymatically degraded hyaluronic acid in the tumor stroma which resulted in normalized interstitial fluid pressure, re-expansion of the vasculature, increased tumor suppression with gemcitabine and prolonged survival time ${ }^{69,70}$.

\subsection{Therapeutic strategies to modulate the tumor stroma}

Since the contribution of the tumor stroma to the progression of PDAC has been highlighted, a number of strategies have been under intense investigation to disrupt or modulate the tumor stroma. The hedgehog pathway is known to support the tumor stroma via paracrine signaling from neoplastic to stromal cells 68. Therefore, Olive et al. ${ }^{68}$ investigated the effects of a stroma depleting hedgehog inhibitor IPI-926 on the delivery and efficacy of gemcitabine. This combination therapy increased the intra-tumoral vascular density as well as the intra-tumoral concentration of gemcitabine, resulting is a transient stabilization 
of the disease ${ }^{68}$. However, a follow up phase II clinical trial with IPI-926 was discontinued due to increased mortality ${ }^{73}$. Similar to IPI-926, other stromadepleting therapeutic strategies did not improve patient survival and in some cases were associated with toxicity. Özdemir et al. performed a study in which $\alpha$-SMA expressing myofibroblasts were depleted in transgenic mice, resulting in invasive, undifferentiated tumors with enhanced hypoxia, epithelial-tomesenchymal transition, cancer stem cells and reduced survival ${ }^{74}$. Additionally, Rhim et al. ${ }^{75}$ deleted sonic hedgehog in a in a mouse model of PDAC, resulting in tumors with reduced stroma content. These tumors were more aggressive, exhibited undifferentiated histology, increased vascularity and heightened proliferation compared to controls ${ }^{75}$.

One the one hand, these findings brought up the theory that the stroma has tumor suppressive properties. On the other hand, the negative outcome of stroma depleting studies might be due to the complete removal of fibrotic barriers which hold tumor cells in place and prevents their metastasis. Therefore, modulating the tumor stroma to dampen its' tumor promoting activities rather than depleting the stroma could result in therapeutic benefits ${ }^{38}$. More recently, the benefits of this strategy have been demonstrated by us and others.

The PEGylated hyaluronidase (PEGPH20) has been assessed in combination with gemcitabine, improving survival and attenuating tumor growth in mice when compared with gemcitabine alone ${ }^{69}$. In a phase Ib study, PEGPH20 in combination with gemcitabine showed an increase in progression-free and overall survival rates of patients with metastatic PDAC, but also thromboembolic event in $29 \%$ of patients ${ }^{76}$. PEGPH20 is currently tested in advanced clinical trials.

Inhibition of MMPs is another therapeutic strategy for the treatment of PDAC. Marimastat, a multifamily MMP inhibitor, was assessed in a randomized clinical trial in which the 1-year survival rate of patients treated with marimastat was similar to those treated with gemcitabine ${ }^{77}$. However, when marimastat was tested as a combination therapy with gemcitabine, it showed no additional benefits compared to gemcitabine ${ }^{78}$. Bay 12-9566, an inhibitor of MMP-3, -9 and -13 , has also been compared to gemcitabine in a phase III clinical trial but showed less therapeutic efficacy in advanced PDAC ${ }^{79}$.

Connective tissue growth factor, which is known to induce PSC proliferation, migration and ECM production, is another potential therapeutic target ${ }^{80}$. CTGF has been blocked using the monoclonal antibody FG-3019 ${ }^{81}$ or antagonist, 
blocking the interaction between CTGF and chemokine receptors ${ }^{82}$. FG-3019 induced the effectiveness of gemcitabine while not affecting intra-tumoral accumulation of gemcitabine in a mouse model of PDAC ${ }^{81}$.

Pirfenidone, an anti-fibrotic agent, has been shown to reduce PSC proliferation, invasion, migration, secretion of collagen and periostin and decreased overall tumor growth, peritoneal disseminated nodules and liver metastasis in an othotopic mouse model induced with PSCs and cancer cells ${ }^{83}$. When pirfendione treatment was combined with gemcitabine, tumor growth was attenuated compared to gemcitabine alone ${ }^{83}$. Additionally, pirfendione has been used in combination with $\mathrm{N}$-actetyl cysteine and reduced desmoplasia in an orthotopic hamster model, induced with HapT1 pancreatic cancer cells ${ }^{84}$.

Two different inhibitors have been used against angiotensin II, which stimulates proliferation of PSCs through the protein kinase C and EGF-ERK pathway ${ }^{53}$. Olmesartan is an angiotensin II type I receptor blocker, which decreased the proliferation and collagen I synthesis of PSCs and inhibited the growth and $\alpha$ SMA expression in subcutaneous tumors consisting of AsPc-1 and PSCs ${ }^{85}$. Another angiotensin II type I receptor inhibitor, losartan, reduced stress in solid tumors, resulting in increased vascular perfusion which enhanced chemotherapy efficiency in pancreatic and breast cancer models ${ }^{86}$.

Other strategies focus on reprogramming activated PSCs into their quiescent state to diminish PSC-induced tumor promotion ${ }^{53}$. When activated, PSCs lose their cytoplasmic vitamin A (retinol) storing lipid droplets. Patients with PDAC are often deficient in vitamin A and D analogs, which supports the activation of PSCs ${ }^{45,87}$. Treatment of activated PSC in vitro with all-trans retinoic acid (ATRA) showed inhibitory effects on PSC migration and collagen synthesis ${ }^{88}$. Additionally, ATRA treatment of PSCs induced quiescence in PSCs, leading to a reduction in their proliferation and increased apoptosis of surrounding cancer cells ${ }^{89}$. Currently, a phase IB study is underway investigating ATRA along with gemcitabine and nab-paclitaxel in PDAC ${ }^{90}$. Additionally, the vitamin D receptor (VDR) has been shown to be a master transcriptional regulator to regain the quiescent state of PSCs ${ }^{91}$. Calcipotriol, a VDR ligand in combination with gemcitabine, could induce stromal reprogramming in KPC mice, increase drug accumulation in tumors, reduce tumor volume and increase survival compared to gemcitabine treatment alone ${ }^{91}$.

Another interesting class of targets to reprogram PSCs are microRNAs (miRNAs), small non protein coding single stranded RNA molecules, which 
regulate posttranscriptional gene expression ${ }^{35}$. A single miRNA sequence can regulate hundreds of target genes and can thereby miRNAs act as oncogenes or tumor suppressors ${ }^{35}$. Therefore, blocking of oncogenic miRNAs with antisense RNA strands shows therapeutic potential ${ }^{35}$. MicroRNA-21 has been observed to be upregulated in CAFs of PDAC and was associated with poor survival ${ }^{92}$. Although the function of miRNA-21 in the stroma has not been understood, it has shown to reduce the growth of MiaPaCa-2 tumors in mice ${ }^{93}$. In TGF- $\beta$ activated PSCs and PDAC biopsies, miRNA-29a and miRNA-29b were found to be decreased ${ }^{53}$. Restoration of miR-29 expression in PSCs reduced stroma accumulation and tumor growth ${ }^{66}$. In our lab, miRNA-199a and miRNA-214 have been identified to be overexpressed in CAFs and activated PSCs ${ }^{94}$. Additionally, their role in PSC differentiation, migration, tube formation by endothelial cells, PSC-induced paracrine effects on tumor cells and growth of 3D-heterospheroids composed of PSCs and cancer cells has been demonstrated 94 .

Moreover, we have recently found that the endogenous lipid lipoxin A4 (LXA4) is capable to attenuate the activation of human PSCs into CAF-like myofibroblasts in vitro and to reduce fibrosis and tumor growth of stroma-rich subcutaneous tumors in vivo ${ }^{95}$.

\subsection{Targeting strategies for drug delivery to the tumor stroma}

The targeted delivery of potential therapeutic agents into their target cells has the potential to enhance the therapeutic efficacy of drugs and at the same time reduce off-target effects. In general, targeting strategies include direct modification of a drug or modification of a drug delivery system (e.g. liposomes or polymeric nanoparticles), which minimize toxicity, increase bioavailability and/or improve the pharmacology of a drug ${ }^{96}$. There are two different strategies for drug targeting: active and passive targeting. Passive targeting is based on the accumulation of drugs at the target site based on the blood flow, pathophysiological characteristics of the organ and physicochemical characteristics of the drug ${ }^{97}$. To achieve passive targeting, drug or drug delivery systems need to have a prolonged circulation time which can only be achieved if they can escape clearance by the reticuloendothelial system (RES) ${ }^{97}$. Therefore, drug delivery systems and imaging applications are often modified with polyethylene glycol (PEG) at their surface, which results in reduced uptake by the RES, an increased circulation time and can prevent the aggregation and 
association with serum proteins ${ }^{97,98}$. These modifications allow drug delivery systems to reach their target site via fenestration in leaky and highly permeable blood vessels with poor lymphatic drainage, which are commonly present in rapidly expanding solid tumors ${ }^{97}$. The described phenomenon is commonly known as the enhanced permeability and retention (EPR) effect and applicable for nanoparticles or macromolecules with a hydrodynamic size below $200 \mathrm{~nm}^{99-}$ ${ }^{101}$. Active targeting is based on the interaction of a ligand on the drug or drug delivery system and its respective targeting receptor or biomolecule on the surface of a target cell ${ }^{97}$. By this mechanism, the ligand modified drug or drug carrier associates and is taken up into the target cell. Active targeting can additionally be achieved by the physicochemical characteristics of the drug, including size, shape and charge, which can cause increased tissue penetration and cellular uptake ${ }^{97,99}$.

The pathogenic structure of the tumor microenvironment negatively affects the major and most important routes of molecular transport, which are highly efficient in healthy organs such as vascular, trans-vascular and interstitial transport and cellular uptake, preventing drug penetration and efficacy in the tumor and hence drug delivery to CAFs or activated pancreatic stellate cells ${ }^{97}$.

The challenges to achieve drug delivery to activated PSCs in the tumor stroma include overcoming the barrier of morphologically abnormal blood vessel basement membrane in the tumor vasculature ${ }^{102}$ and infiltrated immune cells with a high phagocytic capacity in the vascular area ${ }^{103}$. To overcome these barriers and improve drug delivery to the tumor stroma, strategies as solid stress attenuation, improvement of the tumor vasculature, reduction in the interstitial fluid pressure and improvement of the physicochemical characteristics of therapeutic agents have been applied ${ }^{104,105}$. However, specific and active drug delivery to CAFs or activated PSCs in the complex and heterogeneous tumor stroma is still evolving.

For example, Bansal et al. have synthesized a PDGFR- $\beta$ specific interferon gamma (IFN $\gamma$ ) construct (PPB-HAS- IFN $\gamma$ ) which delivered IFN $\gamma$ to fibroblasts and pericytes and inhibited angiogenesis and tumor growth in melanoma model. In a very recent study, doxorubicin carrying cell penetrating peptide nanoparticles were modified with a monoclonal antibody against fibroblast activation protein- $\alpha$ (FAP- $\alpha$ ), expressed on CAFs, and increased the cellular uptake and efficacy of doxorubicin ${ }^{96}$. In a Pan02 mouse model for pancreatic cancer a conjugate of docetaxel, PEG and acetylated carboxymethylcellulose, 
accumulated in CAFs, reduced metastatic potential and increased the survival of Pan02 bearing mice ${ }^{106}$.

\subsection{Aim of the thesis}

The aim of this thesis is to identify novel therapeutic targets and potential therapeutics to dampen the tumor promoting functions of PSCs in PDAC. Additionally, we focus on the identification of targets for drug delivery and imaging and the design of targeting ligands for this purpose.

To achieve this, we aimed to:

- develop novel cell penetrating peptide-based nanoparticles for the specific delivery of miRNA to human PSCs (hPSC) to inhibit their activation

- investigate the effect of the bioactive lipid, lipoxin A4 (LXA4), on hPSC activation, tumor growth and ECM deposition

- investigate the role of integrin alpha 11 (ITGA11) as a therapeutic target in hPSCs

- design a novel peptide to target ITGA11 (AXI) and study its binding affinity and specificity to ITGA11 on hPSCs and potential for targeted drug delivery to ITGA11 expressing hPSCs

\subsection{Outline of the thesis}

Chapter 2 highlights dysregulated miRNAs in the cell types present in the tumor stroma and their functions in the regulation of the multifaceted tumor stroma. We introduce how the expression of miRNAs can be controlled via the administration of miRNA inhibitors (antagomiR) or miRNA agonists (miR mimics). Additionally, we discuss various miRNA delivery approaches to deliver miRNA inhibitors or agonists, with therapeutic potential, into stromal cells.

In chapter 3, we describe how we targeted anti-miRNA-oligonucleotides (AMOs) to hPSCs to block their tumor promoting effects using a novel cell penetrating peptide (CPP)-based targeting approach, in which CPPs form nanocomplexes (NCs) with anti-miRNA-oligonucleotides. When we compared the transfection efficiency of these NCs in hPSCs, normal human fibroblasts and different human pancreatic tumor cells (Panc-1, MiaPaCa-2 and AsPc-1), we found that our NCs preferentially internalized into hPSCs. Next, we investigated the uptake mechanisms responsible for the preferential uptake of our NCs by 
hPSCs. Finally, the inhibitory effects of anti-miR-199a on hPSCs and hPSC paracrine effect on tumor cells was demonstrated in 2D and 3D cell cultures.

Chapter 4 shows how we investigated the ability of LXA4 to reprogram the tumor stroma. We demonstrated that the LXA4 specific receptor FPR2/ALX is overexpressed in activated hPSCs. Next, hPSCs were treated with LXA4, which inhibited their activation, migration and PSC-induced paracrine effect on pancreatic cancer cells. The inhibitory effects of LXA4 on growth and extracellular matrix (ECM) deposition were shown in tumor-mimicking stromarich 3D-heterospheroids. Eventually, LXA4 demonstrated therapeutic efficacy in vivo, in a stroma-rich co-injection tumor model, in which LXA4 treatment reduced tumor growth and intra-tumoral ECM deposition.

Chapter 5 provides a general introduction of integrins and integrin-mediated signaling, an overview of integrin expression in fibrosis-related cell types and the interaction between integrins and growth factor receptors. Next, the role of integrins in wound healing, fibrosis-driven tumors, tumor metastasis and fibrosis is described. Finally, we focus on novel therapies based on integrin inhibition, including clinical developments, and drug delivery strategies to target integrins in the context of wound healing, fibrosis and tumor stroma.

In chapter 6, we describe the very first examination of the global and cellular expression of ITGA11 in human samples of PDAC. We found that ITGA11 is mostly expressed on $\alpha$-SMA positive cells (CAFs and activated hPSC) in human PDAC. In vitro, we have shown the expression of integrin $\alpha 11$ on activated hPSCs and demonstrated the significance of ITGA11 in TGF- $\beta$ and Panc-1 conditioned medium mediated hPSC activation, cell growth, migration and hPSC-induced paracrine effects on Panc-1 tumor cell migration and invasion.

In chapter 7, we depict the staining of human PDAC and various other human organs for ITGA11 and introduce ITGA11 as a drug delivery target, based on its specific expression in the tumor stroma of PDAC. Next, we designed a novel targeting peptide (AXI) specifically binding to recombinant ITGA11 or ITGA11 on hPSCs in vitro. We then use AXI for the surface-modification of liposomes which facilitates their delivery into ITGA11 expressing hPSCs in vitro. In stroma-rich subcutaneous tumors in mice, AXI-modified liposomes showed increased tumor accumulation, penetration depth and co-localization with ITGA11 expressing cells. 
In chapter 7, we summarize the finding of the research presented in this dissertation and discuss how these results can contribute to the development of targeted strategies to reprogram or modulate the tumor stroma of PDAC.

\subsection{References}

1 Morgagni, G. B. Founders of Modern Medicine: Giovanni Battista Morgagni. (1682-1771). Medical library and historical journal 1, 270277 (1903).

2 Chiaravalli, M., Reni, M. \& O'Reilly, E. M. Pancreatic ductal adenocarcinoma: State-of-the-art 2017 and new therapeutic strategies. Cancer treatment reviews 60, 32-43, doi:10.1016/j.ctrv.2017.08.007 (2017).

3 Siegel, R. L., Miller, K. D. \& Jemal, A. Cancer statistics, 2018. CA: $a$ cancer journal for clinicians 68, 7-30, doi:10.3322/caac.21442 (2018). http://pancreatica.org/pancreatic-cancer/pancreatic-cancerprognosis/, 2018).

5 Ansari, D. et al. Pancreatic cancer: yesterday, today and tomorrow. Future oncology 12, 1929-1946, doi:10.2217/fon-2016-0010 (2016).

6 https://www.cijfersoverkanker.nl/. 2018).

7 Becker, A. E., Hernandez, Y. G., Frucht, H. \& Lucas, A. L. Pancreatic ductal adenocarcinoma: risk factors, screening, and early detection. World journal of gastroenterology 20, 11182-11198, doi:10.3748/wjg.v20.i32.11182 (2014).

8 Nitsche, C. et al. Environmental risk factors for chronic pancreatitis and pancreatic cancer. Digestive diseases 29, 235-242, doi:10.1159/000323933 (2011).

9 Bracci, P. M. Obesity and pancreatic cancer: overview of epidemiologic evidence and biologic mechanisms. Molecular carcinogenesis 51, 5363, doi:10.1002/mc.20778 (2012).

10 Raimondi, S., Lowenfels, A. B., Morselli-Labate, A. M., Maisonneuve, P. \& Pezzilli, R. Pancreatic cancer in chronic pancreatitis; aetiology, incidence, and early detection. Best practice \& research. Clinical gastroenterology 24, 349-358, doi:10.1016/j.bpg.2010.02.007 (2010).

11 Everhart, J. \& Wright, D. Diabetes mellitus as a risk factor for pancreatic cancer. A meta-analysis. Jama 273, 1605-1609 (1995).

12 Canto, M. I. et al. International Cancer of the Pancreas Screening (CAPS) Consortium summit on the management of patients with increased risk for familial pancreatic cancer. Gut 62, 339-347, doi:10.1136/gutjnl-2012-303108 (2013).

13 Goonetilleke, K. S. \& Siriwardena, A. K. Systematic review of carbohydrate antigen (CA 19-9) as a biochemical marker in the diagnosis of pancreatic cancer. European journal of surgical oncology : the journal of the European Society of Surgical Oncology and the British 
Association of Surgical Oncology 33, 266-270, doi:10.1016/j.ejso.2006.10.004 (2007).

14 Duffy, M. J. et al. Tumor markers in pancreatic cancer: a European Group on Tumor Markers (EGTM) status report. Annals of oncology : official journal of the European Society for Medical Oncology 21, 441447, doi:10.1093/annonc/mdp332 (2010).

15 Kulemann, B. et al. Circulating tumor cells found in patients with localized and advanced pancreatic cancer. Pancreas 44, 547-550, doi:10.1097/MPA.0000000000000324 (2015).

16 Ankeny, J. S. et al. Circulating tumour cells as a biomarker for diagnosis and staging in pancreatic cancer. British journal of cancer 114, 13671375, doi:10.1038/bjc.2016.121 (2016).

17 Kulemann, B. et al. Pancreatic cancer: Circulating Tumor Cells and Primary Tumors show Heterogeneous KRAS Mutations. Sci Rep 7, 4510, doi:10.1038/s41598-017-04601-z (2017).

18 Imamura, T. et al. Liquid biopsy in patients with pancreatic cancer: Circulating tumor cells and cell-free nucleic acids. World $J$ Gastroenterol 22, 5627-5641, doi:10.3748/wjg.v22.i25.5627 (2016).

19 Takai, E. \& Yachida, S. Circulating tumor DNA as a liquid biopsy target for detection of pancreatic cancer. World J Gastroenterol 22, 84808488, doi:10.3748/wjg.v22.i38.8480 (2016).

20 Roberts, K. J. et al. A preoperative predictive score of pancreatic fistula following pancreatoduodenectomy. $H P B$ : the official journal of the International Hepato Pancreato Biliary Association 16, 620-628, doi:10.1111/hpb.12186 (2014).

21 Burris, H. A., 3rd et al. Improvements in survival and clinical benefit with gemcitabine as first-line therapy for patients with advanced pancreas cancer: a randomized trial. Journal of clinical oncology : official journal of the American Society of Clinical Oncology 15, 24032413, doi:10.1200/JCO.1997.15.6.2403 (1997).

22 Moore, M. J. et al. Erlotinib plus gemcitabine compared with gemcitabine alone in patients with advanced pancreatic cancer: a phase III trial of the National Cancer Institute of Canada Clinical Trials Group. Journal of clinical oncology : official journal of the American Society of Clinical Oncology 25, 1960-1966, doi:10.1200/JCO.2006.07.9525 (2007).

23 Conroy, T. et al. FOLFIRINOX versus gemcitabine for metastatic pancreatic cancer. The New England journal of medicine 364, 18171825, doi:10.1056/NEJMoa1011923 (2011).

24 Von Hoff, D. D. et al. Increased survival in pancreatic cancer with nabpaclitaxel plus gemcitabine. The New England journal of medicine 369, 1691-1703, doi:10.1056/NEJMoa1304369 (2013). 
25 Alexakis, N. et al. Current standards of surgery for pancreatic cancer. The British journal of surgery 91, 1410-1427, doi:10.1002/bjs.4794 (2004).

26 Vincent, A., Herman, J., Schulick, R., Hruban, R. H. \& Goggins, M. Pancreatic cancer. Lancet 378, 607-620, doi:10.1016/S01406736(10)62307-0 (2011).

27 Iacobuzio-Donahue, C. A., Velculescu, V. E., Wolfgang, C. L. \& Hruban, R. H. Genetic basis of pancreas cancer development and progression: insights from whole-exome and whole-genome sequencing. Clinical cancer research : an official journal of the American Association for Cancer Research 18, 4257-4265, doi:10.1158/1078-0432.CCR-12-0315 (2012).

28 Rozenblum, E. et al. Tumor-suppressive pathways in pancreatic carcinoma. Cancer research 57, 1731-1734 (1997).

29 Basturk, O. et al. A Revised Classification System and Recommendations From the Baltimore Consensus Meeting for Neoplastic Precursor Lesions in the Pancreas. The American journal of surgical pathology 1730-1741, doi:10.1097/PAS.0000000000000533 (2015).

30 Oldfield, L. E., Connor, A. A. \& Gallinger, S. Molecular Events in the Natural History of Pancreatic Cancer. Trends in cancer 3, 336-346, doi:10.1016/j.trecan.2017.04.005 (2017).

31 Feldmann, G., Beaty, R., Hruban, R. H. \& Maitra, A. Molecular genetics of pancreatic intraepithelial neoplasia. Journal of hepato-biliarypancreatic surgery 14, 224-232, doi:10.1007/s00534-006-1166-5 (2007).

32 Kanda, M. et al. Presence of somatic mutations in most early-stage pancreatic intraepithelial neoplasia. Gastroenterology 142, 730-733 e739, doi:10.1053/j.gastro.2011.12.042 (2012).

33 Wu, J. et al. Recurrent GNAS mutations define an unexpected pathway for pancreatic cyst development. Science translational medicine $\mathbf{3}$, 92ra66, doi:10.1126/scitranslmed.3002543 (2011).

34 Mazur, P. K. \& Siveke, J. T. Genetically engineered mouse models of pancreatic cancer: unravelling tumour biology and progressing translational oncology. Gut 61, 1488-1500, doi:10.1136/gutjnl-2011300756 (2012).

35 Kuninty, P. R., Schnittert, J., Storm, G. \& Prakash, J. MicroRNA Targeting to Modulate Tumor Microenvironment. Front Oncol 6, 3, doi:10.3389/fonc.2016.00003 (2016).

36 Ostman, A. \& Augsten, M. Cancer-associated fibroblasts and tumor growth--bystanders turning into key players. Current opinion in genetics \& development 19,67-73, doi:10.1016/j.gde.2009.01.003 (2009).

37 Kalluri, R. The biology and function of fibroblasts in cancer. Nature reviews. Cancer 16, 582-598, doi:10.1038/nrc.2016.73 (2016). 
38 Prakash, J. Cancer-Associated Fibroblasts: Perspectives in Cancer Therapy. Trends in cancer 2, 277-279, doi:10.1016/j.trecan.2016.04.005 (2016).

39 Watari, N., Hotta, Y. \& Mabuchi, Y. Morphological studies on a vitamin A-storing cell and its complex with macrophage observed in mouse pancreatic tissues following excess vitamin A administration. Okajimas folia anatomica Japonica 58, 837-858 (1982).

$40 \quad$ Ene-Obong, A. et al. Activated pancreatic stellate cells sequester CD8+ $\mathrm{T}$ cells to reduce their infiltration of the juxtatumoral compartment of pancreatic ductal adenocarcinoma. Gastroenterology 145, 1121-1132, doi:10.1053/j.gastro.2013.07.025 (2013).

41 Berchtold, S. et al. Collagen type V promotes the malignant phenotype of pancreatic ductal adenocarcinoma. Cancer letters 356, 721-732, doi:10.1016/j.canlet.2014.10.020 (2015).

42 Di Maggio, F. et al. Pancreatic stellate cells regulate blood vessel density in the stroma of pancreatic ductal adenocarcinoma. Pancreatology : official journal of the International Association of Pancreatology 16, 995-1004, doi:10.1016/j.pan.2016.05.393 (2016).

43 Apte, M. V., Wilson, J. S., Lugea, A. \& Pandol, S. J. A starring role for stellate cells in the pancreatic cancer microenvironment. Gastroenterology 144, 1210-1219, doi:10.1053/j.gastro.2012.11.037 (2013).

44 Ohlund, D. et al. Distinct populations of inflammatory fibroblasts and myofibroblasts in pancreatic cancer. The Journal of experimental medicine 214, 579-596, doi:10.1084/jem.20162024 (2017).

45 Apte, M. V. et al. Periacinar stellate shaped cells in rat pancreas: identification, isolation, and culture. Gut 43, 128-133 (1998).

46 Bachem, M. G. et al. Identification, culture, and characterization of pancreatic stellate cells in rats and humans. Gastroenterology 115, 421432 (1998).

47 Allam, A. et al. Pancreatic stellate cells in pancreatic cancer: In focus. Pancreatology : official journal of the International Association of Pancreatology 17, 514-522, doi:10.1016/j.pan.2017.05.390 (2017).

48 Fu, Y., Liu, S., Zeng, S. \& Shen, H. The critical roles of activated stellate cells-mediated paracrine signaling, metabolism and onco-immunology in pancreatic ductal adenocarcinoma. Molecular cancer 17, 62, doi:10.1186/s12943-018-0815-z (2018).

49 Sinn, M. et al. alpha-Smooth muscle actin expression and desmoplastic stromal reaction in pancreatic cancer: results from the CONKO-001 study. British journal of cancer 111, 1917-1923, doi:10.1038/bjc.2014.495 (2014).

50 Bachem, M. G. et al. Pancreatic carcinoma cells induce fibrosis by stimulating proliferation and matrix synthesis of stellate cells. Gastroenterology 128, 907-921 (2005). 
51 Neesse, A., Algul, H., Tuveson, D. A. \& Gress, T. M. Stromal biology and therapy in pancreatic cancer: a changing paradigm. Gut, doi:10.1136/gutjnl-2015-309304 (2015).

52 Omary, M. B., Lugea, A., Lowe, A. W. \& Pandol, S. J. The pancreatic stellate cell: a star on the rise in pancreatic diseases. The Journal of clinical investigation 117, 50-59, doi:10.1172/JCI30082 (2007).

53 Kota, J., Hancock, J., Kwon, J. \& Korc, M. Pancreatic cancer: Stroma and its current and emerging targeted therapies. Cancer letters 391, 3849, doi:10.1016/j.canlet.2016.12.035 (2017).

54 Lugea, A. \& Waldron, R. T. Exosome-Mediated Intercellular Communication Between Stellate Cells and Cancer Cells in Pancreatic Ductal Adenocarcinoma. Pancreas 46, 1-4, doi:10.1097/MPA.0000000000000686 (2017).

55 Yoshida, N. et al. Kindlin-2 in pancreatic stellate cells promotes the progression of pancreatic cancer. Cancer letters 390, 103-114, doi:10.1016/j.canlet.2017.01.008 (2017).

56 Saha, S. et al. Gold Nanoparticle Reprograms Pancreatic Tumor Microenvironment and Inhibits Tumor Growth. ACS nano 10, 1063610651, doi:10.1021/acsnano.6b02231 (2016).

57 Vonlaufen, A. et al. Pancreatic stellate cells: partners in crime with pancreatic cancer cells. Cancer research 68, 2085-2093, doi:10.1158/0008-5472.CAN-07-2477 (2008).

58 Schnittert, J. et al. Anti-microRNA targeting using peptide-based nanocomplexes to inhibit differentiation of human pancreatic stellate cells. Nanomedicine (Lond), doi:10.2217/nnm-2017-0054 (2017).

59 Cao, F. et al. HES 1 is essential for chemoresistance induced by stellate cells and is associated with poor prognosis in pancreatic cancer. Oncology reports 33, 1883-1889, doi:10.3892/or.2015.3789 (2015).

60 Mantoni, T. S., Lunardi, S., Al-Assar, O., Masamune, A. \& Brunner, T. B. Pancreatic stellate cells radioprotect pancreatic cancer cells through beta1-integrin signaling. Cancer research 71, 3453-3458, doi:10.1158/0008-5472.CAN-10-1633 (2011).

61 Strell, C. et al. Stroma-regulated HMGA2 is an independent prognostic marker in PDAC and AAC. $B r \quad J$ Cancer 117, 65-77, doi:10.1038/bjc.2017.140 (2017).

62 Endo, S. et al. Autophagy inhibition enhances antiproliferative effect of salinomycin in pancreatic cancer cells. Pancreatology : official journal of the International Association of Pancreatology 17, 990-996, doi:10.1016/j.pan.2017.08.009 (2017).

63 McCarroll, J. A. et al. Role of pancreatic stellate cells in chemoresistance in pancreatic cancer. Frontiers in physiology 5, 141, doi:10.3389/fphys.2014.00141 (2014).

64 Hesler, R. A. et al. TGF-beta-induced stromal CYR61 promotes resistance to gemcitabine in pancreatic ductal adenocarcinoma through 
downregulation of the nucleoside transporters hENT1 and hCNT3. Carcinogenesis 37, 1041-1051, doi:10.1093/carcin/bgw093 (2016).

65 Arumugam, T. et al. Epithelial to mesenchymal transition contributes to drug resistance in pancreatic cancer. Cancer research 69, 5820-5828, doi:10.1158/0008-5472.CAN-08-2819 (2009).

66 Kikuta, K. et al. Pancreatic stellate cells promote epithelialmesenchymal transition in pancreatic cancer cells. Biochemical and biophysical research communications 403, 380-384, doi:10.1016/j.bbrc.2010.11.040 (2010).

67 Wang, Z. et al. Pancreatic cancer: understanding and overcoming chemoresistance. Nature reviews. Gastroenterology \& hepatology 8, 2733, doi:10.1038/nrgastro.2010.188 (2011).

68 Olive, K. P. et al. Inhibition of Hedgehog signaling enhances delivery of chemotherapy in a mouse model of pancreatic cancer. Science 324, 1457-1461, doi:10.1126/science.1171362 (2009).

69 Jacobetz, M. A. et al. Hyaluronan impairs vascular function and drug delivery in a mouse model of pancreatic cancer. Gut 62, 112-120, doi:10.1136/gutjnl-2012-302529 (2013).

70 Provenzano, P. P. et al. Enzymatic targeting of the stroma ablates physical barriers to treatment of pancreatic ductal adenocarcinoma. Cancer Cell 21, 418-429, doi:10.1016/j.ccr.2012.01.007 (2012).

71 Stylianopoulos, T. et al. Causes, consequences, and remedies for growth-induced solid stress in murine and human tumors. Proc. Natl. Acad. Sci. U. S. A. 109, 15101-15108, doi:10.1073/pnas.1213353109 (2012).

72 Incio, J. et al. Obesity-Induced Inflammation and Desmoplasia Promote Pancreatic Cancer Progression and Resistance to Chemotherapy. Cancer discovery 6, 852-869, doi:10.1158/2159-8290.CD-15-1177 (2016).

73 Amakye, D., Jagani, Z. \& Dorsch, M. Unraveling the therapeutic potential of the Hedgehog pathway in cancer. Nature medicine 19, 14101422, doi:10.1038/nm.3389 (2013).

74 Ozdemir, B. C. et al. Depletion of Carcinoma-Associated Fibroblasts and Fibrosis Induces Immunosuppression and Accelerates Pancreas Cancer with Reduced Survival. Cancer cell 28, 831-833, doi:10.1016/j.ccell.2015.11.002 (2015).

75 Rhim, A. D. et al. Stromal elements act to restrain, rather than support, pancreatic ductal adenocarcinoma. Cancer cell 25, 735-747, doi:10.1016/j.ccr.2014.04.021 (2014).

76 Hingorani, S. R. et al. Phase Ib Study of PEGylated Recombinant Human Hyaluronidase and Gemcitabine in Patients with Advanced Pancreatic Cancer. Clinical cancer research : an official journal of the American Association for Cancer Research 22, 2848-2854, doi:10.1158/1078-0432.CCR-15-2010 (2016). 
77 Bramhall, S. R. et al. Marimastat as first-line therapy for patients with unresectable pancreatic cancer: a randomized trial. Journal of clinical oncology : official journal of the American Society of Clinical Oncology 19, 3447-3455, doi:10.1200/JCO.2001.19.15.3447 (2001).

78 Bramhall, S. R. et al. A double-blind placebo-controlled, randomised study comparing gemcitabine and marimastat with gemcitabine and placebo as first line therapy in patients with advanced pancreatic cancer. British journal of cancer 87, 161-167, doi:10.1038/sj.bjc.6600446 (2002).

79 Moore, M. J. et al. Comparison of gemcitabine versus the matrix metalloproteinase inhibitor BAY 12-9566 in patients with advanced or metastatic adenocarcinoma of the pancreas: a phase III trial of the National Cancer Institute of Canada Clinical Trials Group. Journal of clinical oncology : official journal of the American Society of Clinical Oncology 21, 3296-3302, doi:10.1200/JCO.2003.02.098 (2003).

80 Charrier, A. \& Brigstock, D. R. Regulation of pancreatic function by connective tissue growth factor (CTGF, CCN2). Cytokine \& growth factor reviews 24, 59-68, doi:10.1016/j.cytogfr.2012.07.001 (2013).

81 Neesse, A. et al. CTGF antagonism with mAb FG-3019 enhances chemotherapy response without increasing drug delivery in murine ductal pancreas cancer. Proc. Natl. Acad. Sci. U. S. A. 110, $12325-$ 12330, doi:10.1073/pnas.1300415110 (2013).

82 Ijichi, H. et al. Inhibiting Cxcr2 disrupts tumor-stromal interactions and improves survival in a mouse model of pancreatic ductal adenocarcinoma. The Journal of clinical investigation 121, 4106-4117, doi:10.1172/JCI42754 (2011).

83 Kozono, S. et al. Pirfenidone inhibits pancreatic cancer desmoplasia by regulating stellate cells. Cancer research 73, 2345-2356, doi:10.1158/0008-5472.CAN-12-3180 (2013).

84 Suklabaidya, S. et al. Characterization and use of HapT1-derived homologous tumors as a preclinical model to evaluate therapeutic efficacy of drugs against pancreatic tumor desmoplasia. Oncotarget 7, 41825-41842, doi:10.18632/oncotarget.9729 (2016).

85 Masamune, A. et al. The angiotensin II type I receptor blocker olmesartan inhibits the growth of pancreatic cancer by targeting stellate cell activities in mice. Scandinavian journal of gastroenterology $\mathbf{4 8 ,}$ 602-609, doi:10.3109/00365521.2013.777776 (2013).

86 Chauhan, V. P. et al. Angiotensin inhibition enhances drug delivery and potentiates chemotherapy by decompressing tumour blood vessels. Nature communications 4, 2516, doi:10.1038/ncomms3516 (2013).

87 Armstrong, T., Walters, E., Varshney, S. \& Johnson, C. D. Deficiencies of micronutrients, altered bowel function, and quality of life during late follow-up after pancreaticoduodenectomy for malignancy. 
Pancreatology : official journal of the International Association of Pancreatology 2, 528-534, doi:10.1159/000066095 (2002).

88 Jaster, R. et al. Regulation of pancreatic stellate cell function in vitro: biological and molecular effects of all-trans retinoic acid. Biochemical pharmacology 66, 633-641 (2003).

89 Froeling, F. E. et al. Retinoic acid-induced pancreatic stellate cell quiescence reduces paracrine Wnt-beta-catenin signaling to slow tumor progression. Gastroenterology 141, 1486-1497, 1497 e1481-1414, doi:10.1053/j.gastro.2011.06.047 (2011).

90 Kocher, H. et al. STAR_PAC: A Phase 1B study repurposing ATRA as stromal targeting agent along with gemcitabine and nab\&\#xac;Paclitaxel for pancreatic cancer. Pancreatology : official journal of the International Association of Pancreatology 16, S4-S5, doi:10.1016/j.pan.2016.04.019.

91 Sherman, M. H. et al. Vitamin D receptor-mediated stromal reprogramming suppresses pancreatitis and enhances pancreatic cancer therapy. Cell 159, 80-93, doi:10.1016/j.cell.2014.08.007 (2014).

92 Kadera, B. E. et al. MicroRNA-21 in pancreatic ductal adenocarcinoma tumor-associated fibroblasts promotes metastasis. PLoS One 8, e71978, doi:10.1371/journal.pone.0071978 (2013).

93 Sicard, F., Gayral, M., Lulka, H., Buscail, L. \& Cordelier, P. Targeting miR-21 for the therapy of pancreatic cancer. Molecular therapy: the journal of the American Society of Gene Therapy 21, 986-994, doi:10.1038/mt.2013.35 (2013).

94 Kuninty, P. R. et al. MicroRNA-199a and -214 as potential therapeutic targets in pancreatic stellate cells in pancreatic tumor. Oncotarget 7, 16396-16408, doi:10.18632/oncotarget.7651 (2016).

95 Schnittert, J., Heinrich, M. A., Kuninty, P. R., Storm, G. \& Prakash, J. Reprogramming tumor stroma using an endogenous lipid lipoxin A4 to treat pancreatic cancer. Cancer letters 420, 247-258, doi:10.1016/j.canlet.2018.01.072 (2018).

$96 \mathrm{Ji}$, T. et al. Peptide assembly integration of fibroblast-targeting and cellpenetration features for enhanced antitumor drug delivery. Advanced materials 27, 1865-1873, doi:10.1002/adma.201404715 (2015).

97 Yazdani, S., Bansal, R. \& Prakash, J. Drug targeting to myofibroblasts: Implications for fibrosis and cancer. Advanced drug delivery reviews 121, 101-116, doi:10.1016/j.addr.2017.07.010 (2017).

98 Mitragotri, S., Burke, P. A. \& Langer, R. Overcoming the challenges in administering biopharmaceuticals: formulation and delivery strategies. Nature reviews. Drug discovery 13, 655-672, doi:10.1038/nrd4363 (2014).

99 Muro, S. Challenges in design and characterization of ligand-targeted drug delivery systems. Journal of controlled release : official journal of 
the Controlled Release Society 164, 125-137, doi:10.1016/j.jconrel.2012.05.052 (2012).

100 Yamashita, F. \& Hashida, M. Pharmacokinetic considerations for targeted drug delivery. Advanced drug delivery reviews 65, 139-147, doi:10.1016/j.addr.2012.11.006 (2013).

101 Bae, Y. H. \& Park, K. Targeted drug delivery to tumors: myths, reality and possibility. Journal of controlled release : official journal of the Controlled Release Society 153, 198-205, doi:10.1016/j.jconrel.2011.06.001 (2011).

102 Baluk, P., Morikawa, S., Haskell, A., Mancuso, M. \& McDonald, D. M. Abnormalities of basement membrane on blood vessels and endothelial sprouts in tumors. The American journal of pathology 163, 1801-1815, doi:10.1016/S0002-9440(10)63540-7 (2003).

103 Miao, L., Lin, C. M. \& Huang, L. Stromal barriers and strategies for the delivery of nanomedicine to desmoplastic tumors. Journal of controlled release : official journal of the Controlled Release Society 219, 192-204, doi:10.1016/j.jconrel.2015.08.017 (2015).

104 Khawar, I. A., Kim, J. H. \& Kuh, H. J. Improving drug delivery to solid tumors: priming the tumor microenvironment. Journal of controlled release : official journal of the Controlled Release Society 201, 78-89, doi:10.1016/j.jconrel.2014.12.018 (2015).

105 Chauhan, V. P., Stylianopoulos, T., Boucher, Y. \& Jain, R. K. Delivery of molecular and nanoscale medicine to tumors: transport barriers and strategies. Annual review of chemical and biomolecular engineering $\mathbf{2}$, 281-298, doi:10.1146/annurev-chembioeng-061010-114300 (2011).

106 Ernsting, M. J. et al. Targeting of metastasis-promoting tumorassociated fibroblasts and modulation of pancreatic tumor-associated stroma with a carboxymethylcellulose-docetaxel nanoparticle. Journal of controlled release : official journal of the Controlled Release Society 206, 122-130, doi:10.1016/j.jconrel.2015.03.023 (2015). 


\title{
Chapter 2 - MicroRNA targeting to modulate tumor microenvironment
}

Praneeth R. Kuninty ${ }^{\mathrm{a}}$, Jonas Schnittert ${ }^{\mathrm{a}}$, Gert Storm ${ }^{\mathrm{a}, \mathrm{b}}$, and Jai Prakash ${ }^{\mathrm{a}}$

${ }^{a}$ Targeted Therapeutics Section, Department of Biomaterials, Science and Technology, MIRA Institute for Biomedical Technology and Technical Medicine, University of Twente, Enschede, Netherlands; ${ }^{b}$ Department of Pharmaceutics, Utrecht University, Utrecht, Netherlands

Published in Front Oncol. 2016 Jan 19; volume 6:3.

\begin{abstract}
Communication between stromal cells and tumor cells initiates tumor growth, angiogenesis, invasion, and metastasis. Stromal cells include cancer-associated fibroblasts, tumor-associated macrophages, pericytes, endothelial cells and infiltrating immune cells. MicroRNAs (miRNAs) in the tumor microenvironment have emerged as key players involved in the development of cancer and its progression. miRNAs are small endogenous non-protein-coding RNAs that negatively regulate the expression of multiple target genes at posttranscriptional level and thereby control many cellular processes. In this review, we provide a comprehensive overview of miRNAs dysregulated in different stromal cells and their impact on the regulation of intercellular crosstalk in the tumor microenvironment. We also discuss the therapeutic significance potential of miRNAs to modulate the tumor microenvironment. Since miRNA delivery is quite challenging and the biggest hurdle for clinical translation of miRNA therapeutics, we review various non-viral miRNA delivery systems that can potentially be used for targeting miRNA to stromal cells within the tumor microenvironment.
\end{abstract}




\subsection{Introduction}

The tumor microenvironment is composed of cancer cells and non-cancerous cells so-called stromal cells such as cancer-associated fibroblasts (CAFs), tumorassociated macrophages (TAMs), pericytes, endothelial cells and infiltrating immune cells ${ }^{1,2}$. Over the last decade, it was well established that stromal cells promote tumor growth, angiogenesis, invasion, and metastasis ${ }^{3,4}$. These effects are observed in breast, pancreatic, liver, brain, ovarianand prostatecancer. Evidence suggests that tumor cells recruit stromal cells by secreting chemokines and growth factors, which educate them to create a tumor-favoring microenvironment ${ }^{4}$. The "educated" stromal cells such as CAFs, endothelial cells, pericytes, TAMs and other immune cells, interact with tumor cells as well as among themselves to stimulate tumor growth, metastasis and development of resistance to chemotherapy ${ }^{3,4}$. Intervening into these interactions within the tumor microenvironment is an interesting strategy to develop novel therapies for cancer treatment.

MicroRNAs (miRNAs) are represented as a novel class of therapeutics, regulating multiple signaling pathways within the tumor microenvironment ${ }^{5}$. miRNAs, a class of small (17-25nt) endogenous non-coding RNAs, regulate gene expression at the posttranscriptional level and thereby control cellular processes such as differentiation, proliferation and migration. ${ }^{6}$. miRNAs have the ability to regulate not only one but hundreds of target genes simultaneously and thereby control multiple signaling pathways ${ }^{7}$. Gene silencing occurs through imperfect/perfect complementary base pairing between a miRNA guide strand and the 3'UTR region of the mRNA, which leads to translational repression or mRNA degradation ${ }^{6,8}$. During cancer initiation and progression, the expression levels of multiple miRNAs are aberrantly up- or down-regulated, resulting in an imbalance of cellular pathways ultimately leading to the attainment of a pathological state. In this article, we highlight dysregulated miRNAs in different tumor stromal cells and their functions in the regulation of the multifaceted tumor microenvironment. The expression of miRNAs can be controlled by administering either miRNA inhibitors (antagomiR) or miRNA agonists (miR mimics). We summarize various miRNA delivery approaches that have been or can potentially be applied to deliver miRNA as therapeutics into stromal cells.

\subsubsection{MicroRNA in the tumor microenvironment}

In recent years, many miRNAs have been identified in different stromal cells of the tumor microenvironment as illustrated in Figure 1. The table 1 summarizes 
the miRNAs in the stromal cells from different cancer types with genes regulated by these miRNAs and their functions.

\subsubsection{Cancer-associated fibroblasts (CAFs)}

CAFs are one of the most abundant cell types in the tumor microenvironment of many solid tumors ${ }^{9,10}$. In response to inflammatory stimuli, quiescent fibroblasts differentiate into activated myofibroblast (CAFs), expressing increased levels of $\alpha$-smooth muscle actin ( $\alpha$-SMA). CAFs secrete numerous cytokines, chemokines, and ECM components which actively participate in tumor progression, invasion and metastasis ${ }^{11-14}$. Differentiation of fibroblasts into a CAF phenotype has been proposed to be regulated at the post-transcriptional level by miRNAs ${ }^{15}$.

Several studies have reported the importance of specific miRNAs in the activation and transdifferentiation of fibroblasts to CAFs and CAF-induced tumorigenic actions ${ }^{16-19}$. miRNA-21 is one of the most common miRNAs that is reported to be induced in tumor cells and CAFs of pancreatic and colorectal tumors $^{19-23}$. Inhibition of miR-21 using antagomiR reduced the migration/invasion of $\mathrm{CAFs}^{20}$. Not only upregulation but also downregulation of certain miRNAs can induce a CAF phenotype. Tang et al., identified downregulated miR-200 as a direct regulator of reprogramming fibroblasts into $\mathrm{CAFs}^{24}$. Downregulation of miR-200 in normal fibroblasts accelerated their migration and invasion potential similar to $\mathrm{CAFs}^{24}$. Bronisz and coworkers identified miR-320 as a downstream regulator of the PTEN (Phosphatase and tensin homolog deleted on chromosome 10) gene that controls cell proliferation and migration in CAFs and was co-expressed in tumor stroma of breast tissue. Loss of PTEN and miR-320 has shown to be involved in the reprogramming of the tumor microenvironment to promote tumor invasion and angiogenesis ${ }^{25}$. Mitra et al., found downregulation of miR-31 and miR-214, and upregulation of miR-155 in their miRNA profiling and reversal of the activities of these miRNA in these patient-derived CAFs reversed their phenotype ${ }^{18}$. This study suggested that miRNA reprograms fibroblasts into CAFs and, therefore, targeting of miRNA in stromal cells could be a therapeutic approach to treat cancer ${ }^{18}$. In other studies, miR-31 and miR-148a were shown to be downregulated in endometrial $\mathrm{CAFs}^{16,17}$. Overexpression of miR-31 or miR-148a in these CAFs impaired their ability to stimulate migration and invasion of endometrial cancer cells, which were linked to the direct targets SATB2 and WNT10B for miR-31 and miR148a, respectively ${ }^{16,17}$. 

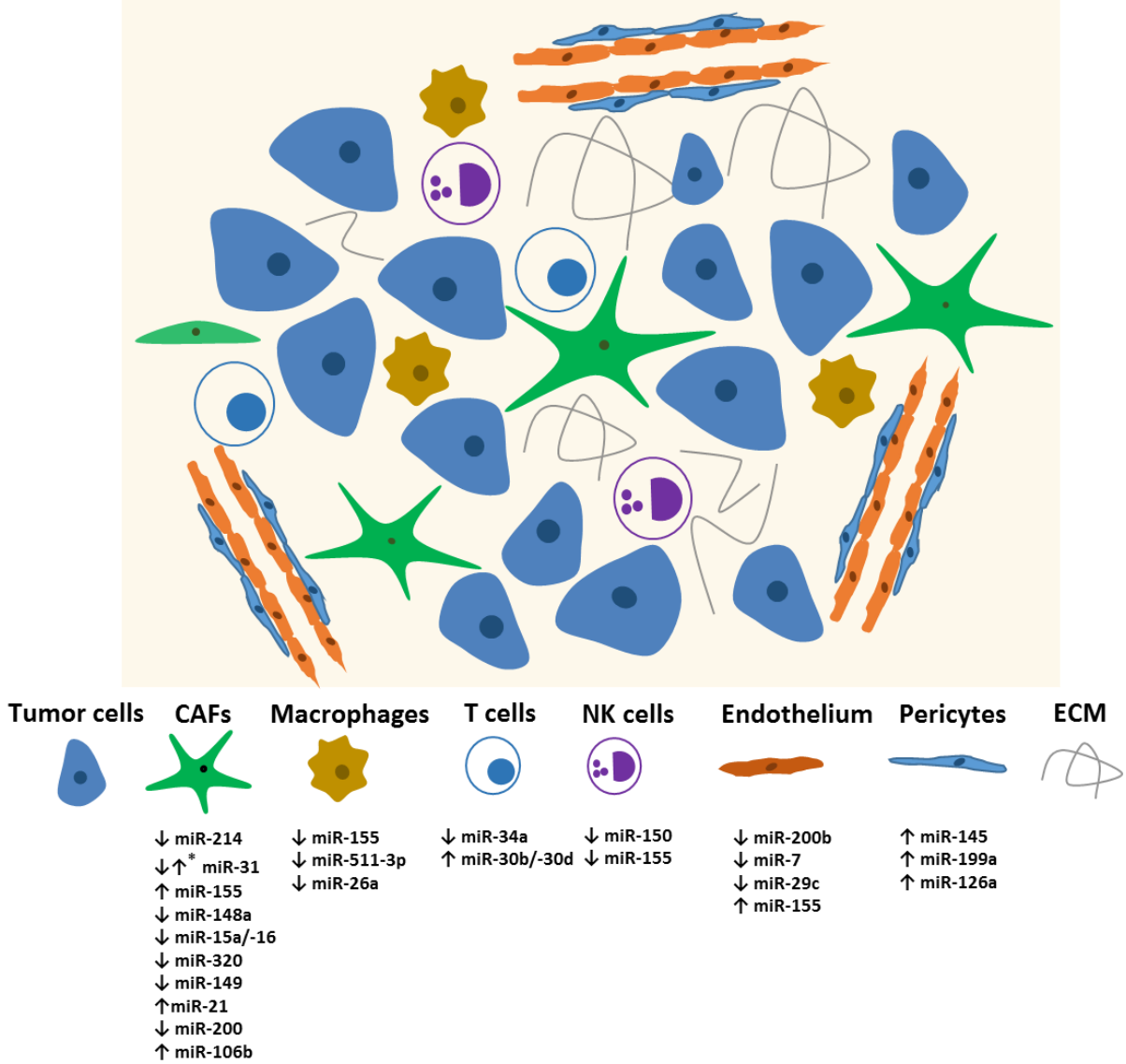

Figure 1. miRNAs mediating changes in tumor microenvironment components. Up and down regulated miRNAs are enlisted. *Mark denotes that the specific miRNA is expressed differentially in different CAFs.

Using miRNA microarray analysis, several dysregulated miRNAs have been identified in breast CAFs e.g. miR-31-3p, 221-5p and 221-3p were upregulated and miR-205, miR-200b, miR-200c, miR-141, miR-101, miR-342-3p, let-7g, and miR-26b were downregulated ${ }^{26,27}$. Furthermore, many miRNA-responsive target genes and signaling pathways were revealed; that regulate different cellular processes such as cell differentiation, adhesion, migration, proliferation, and cell-cell interaction ${ }^{26}$. It is important to note that miR-31 was found to be downregulated in CAFs derived from ovarian and endometrial tumors ${ }^{17,18}$ while it was upregulated in CAFs from breast tumor ${ }^{26}$, indicating that the same miRNA can have dual activities, thereby acting as an oncogene in one tissue and as a tumor suppressor in another. 
In prostate CAFs, miR-15a and miR-16 were shown to be downregulated in CAFs obtained from 23 patients $^{28}$. Downregulation of miR-15 and miR-16 in CAFs promoted tumor progression through the reduced post-transcriptional repression of Fgf-2 and its receptor Fgfr1. These pathways act on both stromal and tumor cells to enhance cancer cell survival, proliferation and migration ${ }^{28}$. Reconstitution of tumor-suppressive miR-15a and miR-16 in CAFs inhibited tumor-promoting ability of stromal cells as shown in a co-injection (tumor cells and CAFs) mouse model, proposing these miRNA as potential targets for the development of novel therapies ${ }^{28}$.

Additionally, Li et al., demonstrated that miR-149 mediates the crosstalk between the tumor cell and CAFs in gastric cancer via prostaglandin E2 (PGE2) and interleukin (IL)-6 signaling pathways ${ }^{29}$. While it remains unclear how PGE2 modulates this crosstalk, it was demonstrated that by targeting IL-6, miR-149 inhibited fibroblast activation ${ }^{29}$. The effects of CAFs on gastric cancer development were negatively regulated by miR-14929. Additionally, CAFs enhanced the epithelial to mesenchymal transition (EMT) and stem-like properties of gastric cancer cells in a miR-149/IL-6 dependent manner ${ }^{29}$. In another study, miR-106b have been identified as a marker of poor prognosis in gastric cancer ${ }^{30}$. Downregulation of miR-106b expression in CAFs resulted in significantly inhibited CAF-induced gastric cancer cell migration and invasion mediated through PTEN pathway ${ }^{30}$. These studies reveal the miRNA targets as diagnostic biomarkers and therapeutic targets for developing the anti-CAF therapy.

\subsubsection{Tumor-associated macrophages and immune cells:}

Macrophages and other immune cells such as T-cells, natural killer (NK) cells are the major inflammatory cells infiltrating into the tumor microenvironment ${ }^{3,31}$. In the past, the infiltration of innate and adaptive immune cells into the tumor microenvironment was considered as an immune attack against cancer ${ }^{31}$. However, now it is widely accepted that immune cells do also promote cancer initiation, progression, and metastasis ${ }^{32}$. Macrophages within the tumor microenvironment can be polarized from anti-tumorigenic M1 macrophages to pro-tumorigenic M2 macrophages (Tumor-associated macrophages (TAMs), via changes in their metabolic pathways and the production of cytokines (CSF-1, IL4, IL-13) by immune cells ${ }^{31}$. TAMs promote tumor progression by stimulating angiogenesis, tumor cell migration and extravasation at metastatic sites and suppressing antitumor immunity thereby reduce patient survival ${ }^{31,33,34}$. Recent studies have unraveled the significance/dysregulation of miRNA in 
macrophages ${ }^{35}$. In a study by Graff et al., miRNA expression profiles were determined in monocyte-derived macrophages differentially polarized into M1, M2a, M2b and M2c phenotypes ${ }^{36}$. They reveal several miRNAs to be uniquely regulated in human macrophages polarized into M1 (miR-125a-3p, miR-26a$2^{*}$ ), M2a (miR-193b) and M2b (miR-27a*, miR-29-b-1*, miR-132, miR$\left.222^{*}\right)^{36}$. Herein we report approaches through which dysregulated miRNAs have been targeted to reprogram miRNA expression in TAMs and thereby suppress their pro-tumorigenic properties.

Squadrito et al., showed that miR-511-3p, encoding for the macrophage mannose receptor, is upregulated in $\mathrm{MRC}^{+}{ }^{+} \mathrm{TAMs}^{37,38}$. Enforcing miR-511-3p expression in $\mathrm{MRC1}^{+} \mathrm{TAMs}$ resulted in the suppression of protumoral genes and inhibited tumor growth with a change in blood vessel morphology. These effects were attributed to ROCK2, a direct target of miR-511-3 $\mathrm{p}^{37}$. The protein expression of transcription factor $\mathrm{C} / \mathrm{EBP} \beta$ showed elevated levels in TAMs as well as human hepatocellular carcinoma tumor sections in situ ${ }^{39}$ C/EBP $\beta$ expression was correlated with the production of cytokines in tumor-activated monocytes and shown to be regulated by sustained reduction of miR-155 $5^{39}$. Overexpression of miRNA-155 was shown to attenuate the production of the cytokines (IL-6, IL10 , TNF- $\alpha$ ) by suppressing $\mathrm{C} / \mathrm{EBP} \beta$ expression which led to inversion of protumoral M2 into pro-inflammatory M1 macrophages, as demonstrated by upregulated M1 markers (TNF- $\alpha$, NOS2, and IL-12) and downregulated M2 markers (Arg1, Ym1, Msr2, Fizz1, and IL-10) ${ }^{40}$. More recently, ectopic expression of miR-26 in hepatocellular carcinoma (HCC) cells suppressed the tumor growth, downregulated the expression of macrophage colony stimulating factor (M-CSF) through the PI3K/Akt pathway, and suppressed the infiltration of macrophages in tumors ${ }^{41}$. In addition, miR-26a expression was inversely correlated with M-CSF expression and the infiltration of macrophages into the tumor tissue of HCC patients ${ }^{41}$.

Besides TAMs, other immune cells such as myeloid-derived suppressor cells (MDSCs), natural killer (NK) cells and $\mathrm{T}$ cells also express miRNAs that regulate their pro-tumorigenic potential. MDSCs negatively regulate immune responses by suppressing the anti-tumor functions of CD4+ and CD8+ T cells by inhibiting the activities of natural killer cells ${ }^{42}$. miR-155 and miR-21 are reported as the most upregulated miRNAs in MDSCs from bone marrow cells, regulating PTEN and SHIP1, respectively ${ }^{43}$. They promote STAT3 activity inducing MDSC expansion that promote tumor aggressiveness via immunosuppression $^{43}$. In HCC, positive for the hepatitis B virus, suppressed 
levels of miR-34a resulted in the enhanced production of chemokine CCL22, thereby recruiting regulatory $\mathrm{T}$ cells (Tregs) into the tumor microenvironment to facilitate immune escape ${ }^{44}$. In human melanoma upregulation of miR-30b/-30d correlates with stage, metastatic potential, shorter time to recurrence and reduced overall survival ${ }^{45}$. Upregulation of miR-30d in the immunocompetent mice triggered immunosuppressive properties at the lung metastatic site, shown by an enhanced infiltration of Tregs ${ }^{45}$. Bezman et al., suggested that miR-150 differentially controls the development of natural killer (NK) and invariant NK $\mathrm{T}$ (iNKT) cells by targeting c-Myb. Mice with miR-150 deletion showed a defect in their ability to develop mature $\mathrm{NK}$ cells ${ }^{46}$. Overexpression of miR-150 promotes the development of mature NK-cells, which were highly responsive to activation $^{46}$. Contrarily, the number of iNKT cells was reduced upon miR-150 upregulation ${ }^{46}$. MiR-155 was found to regulate partly interferon-gamma production in human NK-cells by downregulating SHIP1, making it a potential target in neoplastic disease ${ }^{47}$.

\subsubsection{Tumor vascular cells}

Endothelial cells together with pericytes are the major cellular components of tumor blood vessels, thus playing an important role in angiogenesis during tumor development ${ }^{2-4}$. Chan et al., identified avian erythroblastosis virus E26 (v-ets) oncogene homolog-1 (Ets-1), an angiogenesis-related transcription factor, regulated by miR-200b ${ }^{48}$. Ectopic expression of miR-200b reduced the tube formation and cell migration ability of human microvascular endothelial cells (HMECs) in vitro by targeting Ets- 1 and its associated genes, matrix metalloproteinase-1 and VEGF receptor- $2^{48}$. Interestingly, the authors demonstrated that miR-200b downregulation is hypoxia-induced and represses Ets-1 expression to promote angiogenesis in $\mathrm{HMECs}^{48}$. Hypoxia stimulation also influences several miRNA expression levels in rat cortical pericytes compared to normoxic conditions ${ }^{49}$. Real-time PCR data revealed changes in the expression of miRNAs associated with hypoxia-inducable factor-1 $\alpha$ (HIF-1 $\alpha$ ) (miR-322, miR-199a), TGF- $\beta 1$ (miR-140, miR145, miR-376b-3p) and VEGF (miR-126a, miR-297, miR-16 miR-17-5p) ${ }^{49}$.

In human umbilical vein endothelial cells (HUVECs), miR-29c was identified to regulate cell cycle, proliferation, and angiogenesis in vitro, likely mediated by suppressing Insulin-like growth factor- $1^{50}$. miRNA-7 was identified as a negative regulator of angiogenesis, strongly reducing cell viability, tube formation, sprouting and migration in vitro ${ }^{51}$. In an in vivo murine neuroblastoma tumor model, angiogenesis and tumor growth were significantly inhibited upon 
local administration of miR-7 ${ }^{51}$. MiR-155, which is known to be upregulated in various human cancers, is also involved in the angiogenesis of breast cancer by targeting the von Hippel-Lindau (VHL) tumor suppressor gene ${ }^{52}$. Mammary fat pad xenotransplantation of ectopically expressed miR-155 strongly induced angiogenesis, proliferation, tumor necrosis and recruitment of pro-inflammatory $\mathrm{TAMs}^{52}$. Moreover, miR-155 was identified as a marker for late-stage, lymph node metastasis and poor prognosis in breast cancer ${ }^{52}$.

Recently, miRNA array profiling revealed that endothelial cells communicate with pericytes via miRNA-containing exosomes which increased VEGF-B expression in pericytes at both gene and proteins levels ${ }^{53}$. In addition, Lim et al showed that CXCL12-specific miRNAs are transported from bone marrow stroma to breast cancer cells via gap junctions and reduced CXCL12 levels as well as proliferation ${ }^{54}$. Furthermore, mechanisms for the transfer of siRNA and miRNA through the gap junctions have been reviewed ${ }^{55}$.

\subsection{MicroRNA delivery to the tumor stromal cells}

To turn miRNAs into therapeutics, it is essential to deliver antagomiRs or miRNA mimics cell specifically into the target cells. However, naked miRNAs are unable to pass through cell membrane due to their hydrophilicity, polyanionic nature, and high molecular weight. Intravenous administration of naked miRNA leads to poor tissue distribution due to rapid renal excretion (plasma half-life -1 hour) and degradation by serum RNases ${ }^{56}$. In vivo application of miRNA either requires a chemical modification or formulation into delivery systems. There are several articles describing targeting strategies for miRNA delivery ${ }^{57-64}$. Despite having many strategies reported, the number of successful delivery approaches in vivo is still limited.

\subsubsection{Strategies for miRNA delivery}

Encapsulation of miRNAs into nanoparticles has been addressed to protect miRNAs from degradation by nucleases, resulting in an improved circulating half-life when administered systemically ${ }^{65}$. Viral and non-viral encapsulation strategies have been investigated for this purpose. Viral-based delivery of nucleotides shows high transfection efficiencies, but will not be discussed in this review due to concerns regarding strong inflammatory side effects ${ }^{66}$. The advantages of non-viral delivery systems over viral delivery systems are their well-defined molecular composition, simpler manufacturing and considerably 
lower immunogenicity ${ }^{66}$. The advantages of non-viral delivery systems over viral delivery systems are their well-defined molecular composition, simpler manufacturing and considerably lower immunogenicity ${ }^{67}$.

Non-viral delivery systems that have been investigated for their usability as miRNA carrier systems are liposomes, lipoplexes, and polyplexes. Liposomes are composed of phospholipids possessing a hydrophilic head linked to a hydrophobic tail ${ }^{68}$. This amphiphilic structure allows them to form vesicles with an inner aqueous compartment in which miRNAs can be encapsulated ${ }^{68}$. Cationic lipids have been used to form complexes with negatively charged miRNA, called lipoplexes ${ }^{69}$. Liposomes and lipoplexes have shown to protect miRNAs from degradation by nucleases, resulting in an improved circulating half-life when administered systemically ${ }^{65}$. In addition, cationic polymers that are frequently used for intracellular delivery are polyethyleneimine (PEI) and polyamide amine dendrimers (PAMAM). PEI- and PAMAM-based polyplexes have been used successfully for the delivery of miRNA and in vitro and in $v_{i v o^{70,71}}$. Systemic delivery of PAMAM had toxic effects on the liver and the kidney in mice ${ }^{72}$. For PEI, clinical translation has been hampered by dosedependent toxicity upon systemic administration ${ }^{73}$. Similar toxicity issues can be expected from lipoplexes.

\subsubsection{MicroRNA delivery to tumor stroma cells}

The number of studies for the delivery of miRNAs to the compartments of the tumor stroma in vivo is highly limited. Polyplexes based on polyethyleneimine (PEI) delivered dicer substrate RNA duplexes, mimicking the structure of endogenous precursor miRNA-155 hairpin (Dmi155), into ID8-Defb29/Vegf-A tumors in mice ${ }^{74}$. Increasing the levels of miRNA-155 in tumor-associated dendritic cells silenced multiple immunosuppressive mediators ${ }^{74}$. These changes lead to the transformation of tumor infiltrating dendritic cells from their immunosuppressive phenotype to immunostimulatory cells ${ }^{74}$. Posttransformation of these cells were capable of triggering antitumor responses thereby inhibiting the progression of established ovarian cancers in mice ${ }^{74}$.

Endothelial cells are the only cell type present in the tumor microenvironment, which have been actively targeted in vivo for miRNA delivery. Anti-angiogenic effects in tumors were achieved in a study performed by Ando et al., using lipoplexes modified with a PEG chain bearing the angiogenic vessel targeting peptide Ala-Pro-Arg-Pro-Gly (APRPG ${ }^{75}$. The APRPG-PEG-modified liposomes were used to form complexes with miR-499. The resulting lipoplexes 
were injected into mice bearing Colon 26 NL-17 xenografts ${ }^{75}$. The lipoplexes accumulated in both angiogenic vessels and cancer cells resulting in downregulation of miR-499-regulated proteins and vascular endothelial growth factor (VEGF) ${ }^{75}$. Following an injection of $0.5 \mathrm{mg} / \mathrm{kg}$ of the lipoplexes, tumor growth in mice was significantly inhibited ${ }^{75}$.

In a study by Liu et al., anti-miR-296 antagomiR was delivered into human vein umbilical vein endothelial cells ${ }^{76}$. PEGylated liposome-polycation-hyaluronic acid (LPH) nanoparticles conjugated with a cyclic RGD peptide (cRGD) were used as carrier systems for the specific targeting of $\alpha_{v} \beta_{3}$, a receptor present on endothelial cells in the tumor neovasculature ${ }^{76}$. They reported inhibition of tube formation and endothelial cell migration, and the significant upregulation of hepatocyte growth factor-regulated tyrosine kinase substrate (HGS), one of the genes suppressed by miRNA-296 ${ }^{76}$. A matrigel plug assay was performed to analyze the effect of anti-miR-296 delivery on in vivo angiogenesis ${ }^{76}$. As a result, a decrease in microvessel formation by preventing CD31-positive endothelial cells from invading into the matrigel in combination with an induction of HGS in angiogenic endothelial cells was observed ${ }^{76}$.

Anand et al., aimed to repress neovascularization in tumors by inhibiting miRNA-132 levels via the delivery of anti-miRNA-132 ${ }^{77}$. Liposomal nanoparticles composed of distearoylphosphatidylcholine (DSPC), cholesterol, dioleoylphosphatidylethanolamine (DOPE), distearoylphosphatidylethanolamine (DSPE)-mPEG2000 modified with a DSPE-cyclic RGD peptide, targeting $\alpha_{v} \beta_{3}$ were used as a delivery system ${ }^{77}$. miR132, highly overexpressed in the endothelium of human tumors, mediated loss of p120RasGAP thereby inducing neo-vascularization ${ }^{77}$. By restoring p120RasGAP levels using anti-miR-132 delivery, neovascularization was suppressed, and tumor burdens were decreased in an orthotopic xenograft mouse model of human breast carcinoma, and vessels were maintained in their nonpathological resting state $^{77}$. Systemically administered miRNA7 loaded peptide/polymer-based delivery systems modified with a cRGP ligand targeting the integrins $\alpha v \beta 3$ and $\alpha v \beta 5^{78}$, targeted human glioblastoma xenografts in mice and strongly reduced angiogenesis and tumor proliferation. 
Table 1. List of miRNAs in various tumor stromal cells.

\begin{tabular}{|c|c|c|c|c|}
\hline Cell type & miRNA & Cancer type & $\begin{array}{l}\text { Target } \\
\text { gene }\end{array}$ & Functions \\
\hline \multirow[t]{9}{*}{ CAFs } & $\begin{array}{l}\mathrm{miR}- \\
155 / 214{ }^{18}\end{array}$ & Ovarian & CCL5 & Differentiation \\
\hline & $\operatorname{miR}-31^{17}$ & Endometrial & SATB2 & Migration, Invasion \\
\hline & miR-148a ${ }^{16}$ & Endometrial & WNT10B & Migration \\
\hline & $\begin{array}{l}\operatorname{miR}_{28}-15 /-16 \\
\end{array}$ & Prostate & FGF2 & Migration, Proliferation \\
\hline & $\operatorname{miR}-320^{25}$ & Breast & ETS2 & $\begin{array}{l}\text { Invasion, Blood vessels, } \\
\text { Tumor growth }\end{array}$ \\
\hline & miR-106b ${ }^{30}$ & Gastric & PTEN & Migration, Invasion \\
\hline & miR-200c ${ }^{24}$ & Breast & $\begin{array}{l}\text { Fli-1, } \\
\text { TCF12 }\end{array}$ & Migration, Invasion \\
\hline & miR-149 29 & Gastric & IL-6 & $\begin{array}{l}\text { Differentiation and } \\
\text { Anti-stromal effects on } \\
\text { tumor cells }\end{array}$ \\
\hline & $\operatorname{miR}-21^{21}$ & Colorectal & RECK & Differentiation \\
\hline \multirow[t]{3}{*}{ Macrophages } & miR-155 39 & $\begin{array}{l}\text { Hepatocellular } \\
\text { carcinoma }\end{array}$ & $\mathrm{C} / \mathrm{EBP} \beta$ & $\begin{array}{l}\text { Repolarization towards } \\
\text { M2 phenotype }\end{array}$ \\
\hline & $\begin{array}{l}\operatorname{miR}-511-3 p \\
37\end{array}$ & $\begin{array}{l}\text { Macrophages } \\
\text { Expressing MRC1 }\end{array}$ & ROCK2 & $\begin{array}{l}\text { Tumor growth, } \\
\text { blood vessel } \\
\text { morphology }\end{array}$ \\
\hline & $\operatorname{miR}-26 a^{41}$ & $\begin{array}{l}\text { Hepatocellular } \\
\text { carcinoma }\end{array}$ & M-CSF & $\begin{array}{l}\text { Recruitment of } \\
\text { macrophages }\end{array}$ \\
\hline \multirow[t]{2}{*}{ T cells } & miR-34a ${ }^{44}$ & $\begin{array}{l}\text { Hepatocellular } \\
\text { carcinoma }\end{array}$ & CCL22 & $\begin{array}{l}\text { Infiltration of immune } \\
\text { cells }\end{array}$ \\
\hline & miR-30d ${ }^{45}$ & Melanoma cells & GALNT7 & $\begin{array}{l}\text { Infiltration of Tregs } \\
\text { cells }\end{array}$ \\
\hline NK cells & $\begin{array}{l}\text { miR-150, } \\
\text { miR-155 } \\
46,47\end{array}$ & NK cells & SHIP1 & Activation of NK cells \\
\hline \multirow{4}{*}{$\begin{array}{l}\text { Endothelial } \\
\text { cells }\end{array}$} & miR-200b ${ }^{48}$ & & ETS1 & Blood vessels, migration \\
\hline & $\operatorname{miR}-29 c^{50}$ & & IGF1 & $\begin{array}{l}\text { Angiogenesis, } \\
\text { proliferation }\end{array}$ \\
\hline & $\operatorname{miR}-7{ }^{51}$ & Glioblastoma & & $\begin{array}{l}\text { Cell viability, migration, } \\
\text { Blood vessels }\end{array}$ \\
\hline & miR-155 ${ }^{52}$ & Breast & VHL & $\begin{array}{l}\text { Invasion, migration, } \\
\text { Proliferation, blood } \\
\text { vessels }\end{array}$ \\
\hline
\end{tabular}




\begin{tabular}{|l|l|l|l|}
\hline Pericytes & $\begin{array}{l}\text { miR-145, } \\
\text { miR-126a, } \\
\text { miR-199a } 49\end{array}$ & Fli1 & Migration \\
\hline
\end{tabular}

\subsection{Conclusion}

In recent years, miRNAs have been extensively discovered in the tumor stromal cells either to be used as biomarkers or to show their potential for inhibiting the cellular processes. Undoubtedly, the miRNA field has a high potential to develop novel therapeutics against cancer; however, at the same time development of technologies to deliver miRNAs to specific cells are highly essential to utilize them for therapeutic purposes. With this review, we bring together the fields of tumor biology and miRNA delivery, which will surely benefit both biologists and technology developers.

\subsection{Acknowledgements:}

This work was financially supported by Swedish Research Council, Stockholm, Sweden under the project nr. K7/60501283 (JP). Authors sincerely thank Ruchi Bansal for proof reading this review.

\subsection{References}

1 Hanahan, D. \& Coussens, L. M. Accessories to the crime: functions of cells recruited to the tumor microenvironment. Cancer cell 21, 309-322, doi:10.1016/j.ccr.2012.02.022 (2012).

2 Hanahan, D. \& Weinberg, R. A. Hallmarks of cancer: the next generation. Cell 144, 646-674, doi:10.1016/j.cell.2011.02.013 (2011).

3 Mueller, M. M. \& Fusenig, N. E. Friends or foes - bipolar effects of the tumour stroma in cancer. Nature reviews. Cancer 4, 839-849, doi:10.1038/nrc1477 (2004).

4 Quail, D. F. \& Joyce, J. A. Microenvironmental regulation of tumor progression and metastasis. Nature medicine 19, 1423-1437, doi:10.1038/nm.3394 (2013).

5 Suzuki, H. I., Katsura, A., Matsuyama, H. \& Miyazono, K. MicroRNA regulons in tumor microenvironment. Oncogene 34, 3085-3094, doi:10.1038/onc.2014.254 (2015).

6 Melo, S. A. \& Kalluri, R. Molecular pathways: microRNAs as cancer therapeutics. Clinical cancer research : an official journal of the American Association for Cancer Research 18, 4234-4239, doi:10.1158/1078-0432.CCR-11-2010 (2012). 
7 Esquela-Kerscher, A. \& Slack, F. J. Oncomirs - microRNAs with a role in cancer. Nature reviews. Cancer 6, 259-269, doi:10.1038/nrc1840 (2006).

8 Bader, A. G., Brown, D., Stoudemire, J. \& Lammers, P. Developing therapeutic microRNAs for cancer. Gene therapy 18, 1121-1126, doi:10.1038/gt.2011.79 (2011).

9 Adegboyega, P. A., Mifflin, R. C., DiMari, J. F., Saada, J. I. \& Powell, D. W. Immunohistochemical study of myofibroblasts in normal colonic mucosa, hyperplastic polyps, and adenomatous colorectal polyps. Arch Pathol Lab Med 126, 829-836, doi:10.1043/00039985(2002)126<0829:ISOMIN>2.0.CO;2 (2002).

10 De Wever, O. \& Mareel, M. Role of tissue stroma in cancer cell invasion. The Journal of pathology 200, 429-447, doi:10.1002/path.1398 (2003).

11 Ishii, G., Ochiai, A. \& Neri, S. Phenotypic and functional heterogeneity of cancer-associated fibroblast within the tumor microenvironment. Adv Drug Deliv Rev, doi:10.1016/j.addr.2015.07.007 (2015).

12 Madar, S., Goldstein, I. \& Rotter, V. 'Cancer associated fibroblasts'-more than meets the eye. Trends Mol Med 19, 447-453, doi:10.1016/j.molmed.2013.05.004 (2013).

13 Xing, F., Saidou, J. \& Watabe, K. Cancer associated fibroblasts (CAFs) in tumor microenvironment. Front Biosci (Landmark Ed) 15, 166-179 (2010).

14 Strell, C., Rundqvist, H. \& Ostman, A. Fibroblasts--a key host cell type in tumor initiation, progression, and metastasis. Ups J Med Sci 117, 187195, doi:10.3109/03009734.2012.654859 (2012).

15 Aprelikova, O. \& Green, J. E. MicroRNA regulation in cancerassociated fibroblasts. Cancer Immunol Immunother 61, 231-237, doi:10.1007/s00262-011-1139-7 (2012).

16 Aprelikova, O. et al. Silencing of miR-148a in cancer-associated fibroblasts results in WNT10B-mediated stimulation of tumor cell motility. Oncogene 32, 3246-3253, doi:10.1038/onc.2012.351 (2013).

17 Aprelikova, O. et al. The role of miR-31 and its target gene SATB2 in cancer-associated fibroblasts. Cell Cycle 9, 4387-4398 (2010).

18 Mitra, A. K. et al. MicroRNAs reprogram normal fibroblasts into cancer-associated fibroblasts in ovarian cancer. Cancer discovery $\mathbf{2}$, 1100-1108, doi:10.1158/2159-8290.CD-12-0206 (2012). 
19 Yao, Q. et al. Micro-RNA-21 regulates TGF-beta-induced myofibroblast differentiation by targeting PDCD4 in tumor-stroma interaction. Int J Cancer 128, 1783-1792, doi:10.1002/ijc.25506 (2011).

20 Ali, S. et al. Contribution of microRNAs in understanding the pancreatic tumor microenvironment involving cancer associated stellate and fibroblast cells. Am J Cancer Res 5, 1251-1264 (2015).

21 Bullock, M. D. et al. Pleiotropic actions of miR-21 highlight the critical role of deregulated stromal microRNAs during colorectal cancer progression. Cell Death Dis 4, e684, doi:10.1038/cddis.2013.213 (2013).

22 Kadera, B. E. et al. MicroRNA-21 in pancreatic ductal adenocarcinoma tumor-associated fibroblasts promotes metastasis. PLoS One 8, e71978, doi:10.1371/journal.pone.0071978 (2013).

$23 \mathrm{Li}$, Q. et al. MiR-21/Smad 7 signaling determines TGF-beta1-induced CAF formation. Sci Rep 3, 2038, doi:10.1038/srep02038 (2013).

24 Tang, X. et al. Stromal miR-200s contribute to breast cancer cell invasion through CAF activation and ECM remodeling. Cell Death Differ, doi:10.1038/cdd.2015.78 (2015).

25 Bronisz, A. et al. Reprogramming of the tumour microenvironment by stromal PTEN-regulated miR-320. Nature cell biology 14, 159-167, doi:10.1038/ncb2396 (2012).

26 Zhao, L. et al. MiRNA expression analysis of cancer-associated fibroblasts and normal fibroblasts in breast cancer. Int J Biochem Cell Biol 44, 2051-2059, doi:10.1016/j.biocel.2012.08.005 (2012).

27 Verghese, E. T. et al. MiR-26b is down-regulated in carcinomaassociated fibroblasts from ER-positive breast cancers leading to enhanced cell migration and invasion. The Journal of pathology 231, 388-399, doi:10.1002/path.4248 (2013).

28 Musumeci, M. et al. Control of tumor and microenvironment cross-talk by miR-15a and miR-16 in prostate cancer. Oncogene 30, 4231-4242, doi:10.1038/onc.2011.140 (2011).

29 Li, P. et al. Epigenetic silencing of microRNA-149 in cancer-associated fibroblasts mediates prostaglandin E2/interleukin-6 signaling in the tumor microenvironment. Cell Res 25, 588-603, doi:10.1038/cr.2015.51 (2015).

30 Yang, T. S. et al. MicroRNA-106b in cancer-associated fibroblasts from gastric cancer promotes cell migration and invasion by targeting PTEN. FEBS Lett 588, 2162-2169, doi:10.1016/j.febslet.2014.04.050 (2014). 
31 Fang, H. \& Declerck, Y. A. Targeting the tumor microenvironment: from understanding pathways to effective clinical trials. Cancer research 73, 4965-4977, doi:10.1158/0008-5472.CAN-13-0661 (2013).

32 de Visser, K. E., Eichten, A. \& Coussens, L. M. Paradoxical roles of the immune system during cancer development. Nature reviews. Cancer $\mathbf{6}$, 24-37, doi:10.1038/nrc1782 (2006).

33 Qian, B. Z. \& Pollard, J. W. Macrophage diversity enhances tumor progression and metastasis. Cell 141, 39-51, doi:10.1016/j.cell.2010.03.014 (2010).

34 Chanmee, T., Ontong, P., Konno, K. \& Itano, N. Tumor-associated macrophages as major players in the tumor microenvironment. Cancers (Basel) 6, 1670-1690, doi:10.3390/cancers6031670 (2014).

35 Squadrito, M. L., Etzrodt, M., De Palma, M. \& Pittet, M. J. MicroRNAmediated control of macrophages and its implications for cancer. Trends Immunol 34, 350-359, doi:10.1016/j.it.2013.02.003 (2013).

36 Graff, J. W., Dickson, A. M., Clay, G., McCaffrey, A. P. \& Wilson, M. E. Identifying functional microRNAs in macrophages with polarized phenotypes. $J$ Biol Chem 287, 21816-21825, doi:10.1074/jbc.M111.327031 (2012).

37 Squadrito, M. L. et al. miR-511-3p modulates genetic programs of tumor-associated macrophages. Cell Rep 1, 141-154, doi:10.1016/j.celrep.2011.12.005 (2012).

38 Martinez-Pomares, L. The mannose receptor. Journal of leukocyte biology 92, 1177-1186, doi:10.1189/jlb.0512231 (2012).

39 He, M., Xu, Z., Ding, T., Kuang, D. M. \& Zheng, L. MicroRNA-155 regulates inflammatory cytokine production in tumor-associated macrophages via targeting C/EBPbeta. Cell Mol Immunol 6, 343-352, doi:10.1038/cmi.2009.45 (2009).

40 Cai, X. et al. Re-polarization of tumor-associated macrophages to proinflammatory M1 macrophages by microRNA-155. J Mol Cell Biol 4, 341-343, doi:10.1093/jmcb/mjs044 (2012).

41 Chai, Z. T. et al. microRNA-26a suppresses recruitment of macrophages by down-regulating macrophage colony-stimulating factor expression through the PI3K/Akt pathway in hepatocellular carcinoma. Journal of hematology \& oncology 8, 56, doi:10.1186/s13045-015-0150-4 (2015).

42 Gabrilovich, D. I., Ostrand-Rosenberg, S. \& Bronte, V. Coordinated regulation of myeloid cells by tumours. Nat Rev Immunol 12, 253-268, doi:10.1038/nri3175 (2012). 
43 Li, L. et al. MicroRNA-155 and MicroRNA-21 promote the expansion of functional myeloid-derived suppressor cells. J Immunol 192, 10341043, doi:10.4049/jimmunol.1301309 (2014).

44 Yang, P. et al. TGF-beta-miR-34a-CCL22 signaling-induced Treg cell recruitment promotes venous metastases of $\mathrm{HBV}$-positive hepatocellular carcinoma. Cancer cell 22, 291-303, doi:10.1016/j.ccr.2012.07.023 (2012).

45 Gaziel-Sovran, A. et al. miR-30b/30d regulation of GalNAc transferases enhances invasion and immunosuppression during metastasis. Cancer cell 20, 104-118, doi:10.1016/j.ccr.2011.05.027 (2011).

46 Bezman, N. A., Chakraborty, T., Bender, T. \& Lanier, L. L. miR-150 regulates the development of NK and iNKT cells. J Exp Med 208, 27172731, doi:10.1084/jem.20111386 (2011).

47 Trotta, R. et al. miR-155 regulates IFN-gamma production in natural killer cells. Blood 119, 3478-3485, doi:10.1182/blood-2011-12-398099 (2012).

48 Chan, Y. C., Khanna, S., Roy, S. \& Sen, C. K. miR-200b targets Ets-1 and is down-regulated by hypoxia to induce angiogenic response of endothelial cells. J Biol Chem 286, 2047-2056, doi:10.1074/jbc.M110.158790 (2011).

49 Truettner, J. S., Katyshev, V., Esen-Bilgin, N., Dietrich, W. D. \& DoreDuffy, P. Hypoxia alters MicroRNA expression in rat cortical pericytes. Microrna 2, 32-44, doi:10.2174/2211536611302010005 (2013).

$50 \mathrm{Hu}, \mathrm{Y}$. et al. Evaluation of miR-29c inhibits endotheliocyte migration and angiogenesis of human endothelial cells by suppressing the insulin like growth factor 1. Am J Transl Res 7, 866-877 (2015).

51 Babae, N. et al. Systemic miRNA-7 delivery inhibits tumor angiogenesis and growth in murine xenograft glioblastoma. Oncotarget 5, 6687-6700 (2014).

52 Kong, W. et al. Upregulation of miRNA-155 promotes tumour angiogenesis by targeting VHL and is associated with poor prognosis and triple-negative breast cancer. Oncogene 33, 679-689, doi:10.1038/onc.2012.636 (2014).

53 Yamamoto, S. et al. Inflammation-induced endothelial cell-derived extracellular vesicles modulate the cellular status of pericytes. Sci Rep 5, 8505, doi:10.1038/srep08505 (2015).

54 Lim, P. K. et al. Gap junction-mediated import of microRNA from bone marrow stromal cells can elicit cell cycle quiescence in breast cancer 
cells. Cancer Res 71, 1550-1560, doi:10.1158/0008-5472.CAN-102372 (2011).

55 Brink, P. R., Valiunas, V., Gordon, C., Rosen, M. R. \& Cohen, I. S. Can gap junctions deliver? Biochim Biophys Acta 1818, 2076-2081, doi:10.1016/j.bbamem.2011.09.025 (2012).

56 Wang, J., Lu, Z., Wientjes, M. G. \& Au, J. L. Delivery of siRNA therapeutics: barriers and carriers. The AAPS journal 12, 492-503, doi:10.1208/s12248-010-9210-4 (2010).

57 Gandhi, N. S., Tekade, R. K. \& Chougule, M. B. Nanocarrier mediated delivery of siRNA/miRNA in combination with chemotherapeutic agents for cancer therapy: current progress and advances. $J$ Control Release 194, 238-256, doi:10.1016/j.jconrel.2014.09.001 (2014).

58 Li, Z. \& Rana, T. M. Therapeutic targeting of microRNAs: current status and future challenges. Nature reviews. Drug discovery 13, 622-638, doi:10.1038/nrd4359 (2014).

59 Kortylewski, M. \& Nechaev, S. How to train your dragon: targeted delivery of microRNA to cancer cells in vivo. Molecular therapy : the journal of the American Society of Gene Therapy 22, 1070-1071, doi:10.1038/mt.2014.73 (2014).

60 Kwekkeboom, R. F., Lei, Z., Doevendans, P. A., Musters, R. J. \& Sluijter, J. P. Targeted delivery of miRNA therapeutics for cardiovascular diseases: opportunities and challenges. Clinical science 127, 351-365, doi:10.1042/CS20140005 (2014).

61 Krutzfeldt, J. et al. Silencing of microRNAs in vivo with 'antagomirs'. Nature 438, 685-689, doi:10.1038/nature04303 (2005).

62 Obad, S. et al. Silencing of microRNA families by seed-targeting tiny LNAs. Nat Genet 43, 371-378, doi:10.1038/ng.786 (2011).

63 Lennox, K. A. \& Behlke, M. A. Chemical modification and design of anti-miRNA oligonucleotides. Gene Ther 18, 1111-1120, doi:10.1038/gt.2011.100 (2011).

64 Stenvang, J., Petri, A., Lindow, M., Obad, S. \& Kauppinen, S. Inhibition of microRNA function by antimiR oligonucleotides. Silence 3, 1, doi:10.1186/1758-907X-3-1 (2012).

65 Garzon, R., Marcucci, G. \& Croce, C. M. Targeting microRNAs in cancer: rationale, strategies and challenges. Nature reviews. Drug discovery 9, 775-789, doi:10.1038/nrd3179 (2010). 
66 Choi, Y. S. et al. The systemic delivery of siRNAs by a cell penetrating peptide, low molecular weight protamine. Biomaterials 31, 1429-1443, doi:10.1016/j.biomaterials.2009.11.001 (2010).

67 Park, T. G., Jeong, J. H. \& Kim, S. W. Current status of polymeric gene delivery systems. Adv Drug Deliv Rev 58, 467-486, doi:10.1016/j.addr.2006.03.007 (2006).

68 Tros de Ilarduya, C., Sun, Y. \& Duzgunes, N. Gene delivery by lipoplexes and polyplexes. European journal of pharmaceutical sciences : official journal of the European Federation for Pharmaceutical Sciences 40, 159-170, doi:10.1016/j.ejps.2010.03.019 (2010).

69 Ben-Shushan, D. et al. Overcoming obstacles in microRNA delivery towards improved cancer therapy. Drug Deliv. and Transl. Res. 4, 3849, doi:10.1007/s13346-013-0160-0 (2014).

70 Ren, Y. et al. Co-delivery of as-miR-21 and 5-FU by poly(amidoamine) dendrimer attenuates human glioma cell growth in vitro. Journal of biomaterials science. Polymer edition 21, 303-314, doi:10.1163/156856209X415828 (2010).

71 Zhang, Y., Wang, Z. \& Gemeinhart, R. A. Progress in microRNA delivery. $J$ Control Release 172, 962-974, doi:10.1016/j.jconrel.2013.09.015 (2013).

72 Chauhan, A. S., Jain, N. K. \& Diwan, P. V. Pre-clinical and behavioural toxicity profile of PAMAM dendrimers in mice. (2009).

73 Zhang, Y., Satterlee, A. \& Huang, L. In vivo gene delivery by nonviral vectors: overcoming hurdles? Mol Ther 20, 1298-1304, doi:10.1038/mt.2012.79 (2012).

74 Cubillos-Ruiz, J. R. et al. Reprogramming tumor-associated dendritic cells in vivo using miRNA mimetics triggers protective immunity against ovarian cancer. Cancer research 72, 1683-1693, doi:10.1158/0008-5472.CAN-11-3160 (2012).

75 Ando, H. et al. Advanced cancer therapy by integrative antitumor actions via systemic administration of miR-499. J Control Release 181, 32-39, doi:10.1016/j.jconrel.2014.02.019 (2014).

76 Liu, X. Q., Song, W. J., Sun, T. M., Zhang, P. Z. \& Wang, J. Targeted delivery of antisense inhibitor of miRNA for antiangiogenesis therapy using cRGD-functionalized nanoparticles. Molecular pharmaceutics $\mathbf{8}$, 250-259, doi:10.1021/mp100315q (2011). 
77 Anand, S. et al. MicroRNA-132-mediated loss of p120RasGAP activates the endothelium to facilitate pathological angiogenesis. Nature medicine 16, 909-914, doi:10.1038/nm.2186 (2010).

78 Chou, S. T. et al. Surface-modified HK:siRNA nanoplexes with enhanced pharmacokinetics and tumor growth inhibition. Biomacromolecules 14, 752-760, doi:10.1021/bm3018356 (2013). 


\section{Chapter 3 - Anti-microRNA targeting using peptide-based nanocomplexes to inhibit differentiation of human pancreatic stellate cells}

Jonas Schnittert ${ }^{\mathrm{a}}$, Praneeth R. Kuninty ${ }^{\mathrm{a}}$, Tomasz Bystry ${ }^{\mathrm{a}}$, Roland Brock ${ }^{\mathrm{b}}$, Gert Storm $^{\mathrm{a}, \mathrm{c}}$, Jai Prakash ${ }^{\mathrm{a}, *}$

a Department of Biomaterials, Science and Technology, Section: Targeted Therapeutics, MIRA Institute for Biomedical Technology and Technical Medicine, University of Twente, Enschede, The Netherlands. ${ }^{b}$ Department of Biochemistry, Radboud University Medical Center, Nijmegen, The Netherlands. ${ }^{c}$ Department of Pharmaceutics, Utrecht Institute for Pharmaceutical Sciences (UIPS), Utrecht University, Universiteitsweg 99, 3584 CG Utrecht, The Netherlands

Published in Nanomedicine (Lond). 2017

\section{Abstract}

Aim: To develop novel peptide-based nanocomplexes (NCs) for delivery of antimiRNA oligonucleotides to human-derived pancreatic stellate cells (hPSCs), precursors of cancer-associated fibroblasts. Materials \& methods: NCs of antimiRNA oligonucleotides and cell-penetrating peptides (different variants) were formed and characterized. The effects of anti-miR-199a delivery on hPSC differentiation and 3D heterospheroid formation were investigated. Results: Dimeric cell-penetrating peptide based NCs (NC-2) showed 130-fold higher uptake by hPSCs compared with monomer-based NCs (NC-1) and tenfold higher uptake compared with general fibroblasts and different pancreatic tumor cells. Interestingly, delivery of anti-miR-199a inhibited hPSC differentiation into cancer-associated fibroblasts and inhibited the size of 3D heterospheroids comprised of hPSCs and tumor cells. Conclusion: Our NCs present a highly efficient anti-miRNA delivery system to hPSCs to inhibit their protumorigenic activity. 


\section{Graphical abstract}

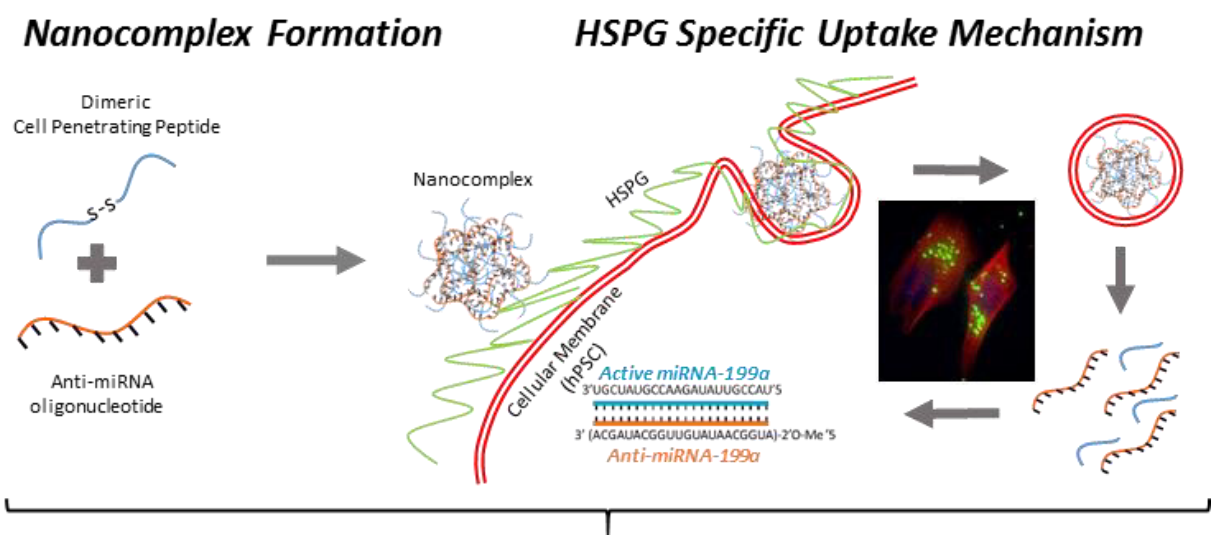

Inhibited CAF differentiation and 3D-spheroid growth
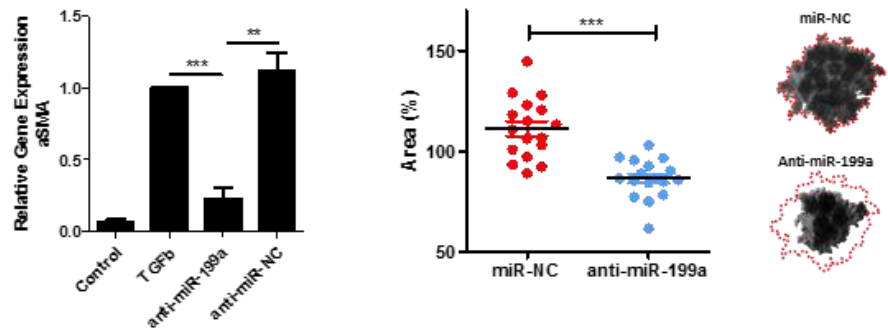

In this study, we developed novel cell-penetrating peptide based nanocomplexes $(\sim 40 \mathrm{~nm})$ to efficiently deliver anti-miRNA-199a to primary human pancreatic stellate cells (hPSCs). Our nanocomplexes significantly inhibited the differentiation of hPSCs into tumor-promoting human pancreatic tumor stromal myofibroblasts and reduced the growth of in vitro tumor heterospheroids composed of hPSCs and Panc-1 epithelial cancer cells.

Keywords: cancer-associated fibroblasts, cell-penetrating peptides, microRNA199a, miRNA delivery, nanocomplexes, pancreatic cancer, tumor microenvironment 


\subsection{Introduction}

miRNAs are nonprotein-coding ssRNA molecules, ranging from 19 to 25 nucleotides, which regulate posttranscriptional mRNA expression via complementary binding to their target mRNA. By this mechanism, miRNAs possess the ability to control hundreds of target genes simultaneously and thereby regulate cellular pathways involved in cell survival, proliferation, differentiation, apoptosis and metabolism ${ }^{1-3}$. In a pathological state, such as cancer, the expression levels of various miRNAs are greatly altered, contributing to the imbalance between cellular pathways and thereby attainment of the pathological state ${ }^{1,4}$. By means of miRNA modulators (miRNA mimics and antimiRNA modulators), the pathological state can be inhibited or reversed, making miRNA-based therapeutics an attractive strategy to treat a disease ${ }^{5-8}$. However, the poor pharmacokinetic profile of miRNA oligonucleotides as a consequence of their rapid degradation by serum nucleases, rapid renal elimination and nonspecific distribution limits their systemic applications ${ }^{5-8}$. Furthermore, at the cellular level, oligonucleotides are unable to penetrate cellular membranes due to their highly negative charge. Nanoparticle-based delivery systems through which miRNA oligonucleotides can be protected from degradation and delivered intracellularly are therefore under intense investigation ${ }^{5,-10}$. Various methods based on nonviral vectors, such as liposomes, micelles, polymeric nanoparticles, nanospheres, antibody and cell-penetrating peptides (CPPs), have been investigated for miRNA delivery ${ }^{1,10,11}$. Especially, CPPs are powerful tools for intracellular delivery of different cargos, because of their ability to induce efficient cellular uptake and their low toxicity ${ }^{12,13}$. CPPs are short peptides with a length of 8-30 amino acids ${ }^{14}$. Their positive charge enables them to spontaneously form noncovalent nanocomplexes (NCs) with negatively charged oligonucleotides via electrostatic interactions ${ }^{13,15}$. After coming into contact with cells, these complexes are internalized through various mechanisms, as reviewed by Margus et al. ${ }^{16}$. Pancreatic ductal adenocarcinoma (PDA) is one of the most aggressive cancer types, for which, to date, no treatment has had a significant impact $^{17}$.

PDA is characterized by a rapid progression, early-on metastasis formation and a high resistance to chemo- and radiotherapy ${ }^{18-20}$. PDA possesses a dense desmoplastic reaction surrounding malignant cells, known as tumor stroma ${ }^{19-23}$. A major cell type of the tumor stroma are cancer-associated fibroblasts (CAFs) which in the pancreatic tumor stroma mainly originate from resident pancreatic stellate cells (PSCs) $)^{21,24,25}$. During carcinogenesis, PSCs become activated and 
undergo subsequent functional alterations, resulting in an increased proliferation and migration potential ${ }^{22,26}$. Once activated, PSCs secrete mutagenic substances, which stimulate tumor growth, invasion and resistance to chemo- and radiotherapy ${ }^{21,22}$. Recent evidence suggests that both pancreatic tumor cells and stromal PSCs have dysregulated miRNA expression levels ${ }^{27,28}$.

In this study, we aimed to target anti-miRNA oligonucleotides (AMOs) to inhibit miRNAs in stromal PSCs to block their protumorigenic effects. To deliver AMOs into primary human-derived PSCs (hPSCs), we designed a CPP-based targeting approach, in which CPPs form NCs with oligonucleotides. Nanoscale particles are well known to yield effective tumor delivery due to the enhanced permeability and retention effect. First, we used an arginine-rich $\mathrm{CPP}^{11}$ to form NCs with AMOs and found that these NCs poorly internalized into hPSCs. We hypothesized that this lack of activity might be attributed to the unavailability of the CPP on the surface of the NCs to interact with cells. To allow for direct interaction between CPPs and cells and yet achieve nanocomplex formation with AMO, we designed a disulfide-containing dimeric form of the CPP reasoning that one CPP chain will be complexed with the AMOs and the other CPP chain will be available to interact with the cells. Additionally, the introduction of disulfide bridges into CPPs has shown to result in their cellular association and uptake via disulfide exchange and supports their endosomal escape ${ }^{29,30}$. To explore the value of disulfide-mediated dimerization for the delivery of AMO, we prepared the NCs with monomeric or dimeric CPPs and compared their ability to transfect hPSCs. Furthermore, we also compared the transfection efficiency of these NCs in hPSCs, normal human fibroblasts and different human pancreatic tumor cells (Panc-1, MiaPaCa-2 and AsPc-1). We also investigated the uptake mechanisms of NCs in hPSCs and showed the inhibitory effects of anti-miR-199a in hPSCs in 2D and 3D cultures.

\subsection{Methods}

\subsubsection{Materials}

Scrambled miRNA and anti-miRNA-199a with the respective sequences: 5'CAGUACUUUUGU- GUAGUACAA'3 and 5'UGUCAUCAGACGUGUAACCAAU' 3 with 2'O-methyl modification were purchased from TriLink BioTechnologies (CA, USA). FAM-labeled scrambled AMO was ordered from GenePharma (Shanghai, China). miRNAs were aliquoted in RNase-free water (Sigma Life Science, Steinheim, Germany) to a concentration of $20 \mu \mathrm{M}$. The monomeric form of CPP (VSRRRRRRGGRRRR) 
and dimeric CPP (RRRRGGRRRRRRSV-C-S-S-C-VSRRRRRRGGRRRR) were custom-synthesized from ChinaPeptides (Shanghai, China).

\subsubsection{Formation of NCs}

$\mathrm{NC}$ formation was performed by adding increasing volumes of $100 \mu \mathrm{M}$ monomeric or dimeric CPP solution to $15 \mu \mathrm{l}$ of $20 \mu \mathrm{M}$ miRNA. Next, milli-Q water was added to the mixture to obtain a total volume of $300 \mu$. The solution was mixed properly by resuspension, followed by incubation for $1 \mathrm{~h}$ at room temperature (RT). By this method, NCs with charge ratios from 1:1 to 20:1 ( \pm ) were formed. Charge ratios are calculated on the basis of moles of the positively charged amino acids of the CPP and the negatively charged phosphate groups of miRNA.

\subsubsection{Determination of complex size $\&$ $\zeta$-potential}

The size and zeta-potential of NCs were measured using dynamic light scattering with a Malvern Zetasizer Nano ZS (Malvern instruments, Worcestershire, UK). The complex-containing solution was mixed with $700 \mu$ of Milli-Q water and added to a disposable $1.5 \mathrm{ml}$ cuvette (BrandTech Scientific, CT, USA) for size measurements, or injected into a folded capillary $\zeta$-potential cuvette (BrandTech Scientific) to measure $\zeta$-potentials.

\subsubsection{Gel retardation assay}

To assess the incorporation of miRNA into NCs, a gel retardation assay was performed. For each NC prepared at different charge ratios of CPP and miRNA, $60 \mu \mathrm{l}$ of NC solution was mixed with $20 \mu \mathrm{l}$ glycerol diluted 1:10 in Milli-Q water and separated on a $2 \%$ agarose gel containing $1 \times$ Tris-borate-EDTA (TBE) buffer (Thermo Scientific, IL, USA) for $20 \mathrm{~min}$ at $120 \mathrm{~V}$. miRNA was stained with SYBR ${ }^{\circledR}$ Gold (Invitrogen, CA, USA) according to the manufacturer's instructions. The gels were photographed in a FluorChem M imaging system (ProteinSimple, CA, USA) (excitation filter: $475 \mathrm{~nm}$, emission filter: $537 \mathrm{~nm}$ ).

\subsubsection{Cells}

Cells were grown in a humidified atmosphere at $37^{\circ} \mathrm{C}$ with $5 \% \mathrm{CO}$. Primary hPSCs were maintained in complete Stellate Cell Medium (supplemented with $2 \%$ fetal bovine serum (FBS), $1 \%$ penicillin/streptomycin and 1\% Stellate Cell Growth Supplement (SteCGS; ScienCell, CA, USA). Previous to seeding hPSCs, cell culture flasks and plates were coated with L-lysine (Sigma-Aldrich, Steinheim, Germany) at a concentration of $0.0015(\mathrm{w} / \mathrm{v})$ in $\mathrm{H} 2 \mathrm{O}$, for $1 \mathrm{~h}$ at $37^{\circ} \mathrm{C}$ and washed two-times with Milli-Q water before use. PANC-1 and MiaPaCa-2 
cells were cultured in complete DMEM high glucose ( $4.5 \mathrm{~g} / \mathrm{l})$ with L-glutamine (GE Healthcare, Vienna, Austria) supplemented with 10\% FBS (Lonza, Verviers, Belgium), 100ug/ml peni- cillin/streptomycin (Sigma, Zwijndrecht, The Netherlands). BJ-fibroblasts and AsPc-1-2 cells were cultured in complete RPMI medium (GE Healthcare) supplemented with 10\% FBS (Lonza), 100ug/ml penicillin/ streptomycin (Sigma).

\subsubsection{Cellular uptake of NCs}

\subsubsection{Fluorescence microscopy}

To image cellular uptake, hPSCs, BJ-fibroblasts, Panc- 1, AsPc-1 and MiaPaCa2 cells were seeded into an ibiTreat $\mu$-Slide 8-well plate (Ibidi, Martinsried, Germany) at a seeding density of $1.5 \times 10^{4}$ cells/well, cultured as mentioned above and incubated for $18 \mathrm{~h}$ at $37^{\circ} \mathrm{C}$. After $24 \mathrm{~h}$, the culture medium was replaced with fresh serum-free medium containing NCs with a miRNA-FAM concentration of $200 \mathrm{nM}$. After $6 \mathrm{~h}$ of incubation, cells were washed three-times with DMEM containing 10\% FBS and subsequently fixed in phosphate-buffered saline (PBS) containing 10\% formaldehyde (Sigma) for $15 \mathrm{~min}$. After permeabilizing the cells with $0.1 \mathrm{M}$ Triton X-100 (Sigma) for $5 \mathrm{~min}$, F-actin was stained with phalloidin (Life Technologies, CA, USA) at a concentration of 250 $\mathrm{ng} / \mathrm{ml}$. To stain the nuclei, a drop of mounting medium containing DAPI (Sigma) was added to each well. Cells were imaged for FAM (ex. 495/em. 520), phalloidin (ex. 540/em. 565) and DAPI (ex. 360/em. 460) using an EVOS FI fluorescent microscope (Life Technologies).

\subsubsection{Flow cytometry}

To determine cellular uptake, cells were seeded as mentioned above in 24-well plates at a concentration of $4 \times 10^{4}$ cells/well. For the heparan inhibition studies, cells were incubated with $2 \mu \mathrm{g} / \mathrm{ml}$ heparan lithium salt (Sigma) for $30 \mathrm{~min}$ previous to incubation with NCs. To evaluate the uptake mechanism, cells were incubated with $10 \mu \mathrm{M}$ phenylarsine oxide (Sigma), $50 \mu \mathrm{g} / \mathrm{ml}$ nystatin (Sigma) or $100 \mu \mathrm{M}$ ethylisopropyl amiloride (Sigma) in $500 \mu 1$ of PBS for $10 \mathrm{~min}$ prior to the transfection experiment. After $6 \mathrm{~h}$ of incubation with NCs containing $200 \mathrm{nM}$ fluorescently labeled miRNA, cells were washed twice with PBS and detached with $50 \mu$ trypsin solution (Life Technologies). Trypsin was neutralized by adding $200 \mu \mathrm{l}$ of medium containing $2 \%$ FBS and $200 \mu$ of trypsin neutralizer solution (Life Technologies) to each well. The cell-containing solution was washed twice in $1 \mathrm{ml}$ of ice cold PBS containing $1 \%$ FBS by centrifugation and subsequently fixed in PBS containing $0.5 \%$ formaldehyde (Sigma) previous to analysis with a FACS Calibur (Becton Dickinson, NJ, USA). 


\subsubsection{Cell proliferation assay}

To monitor the cell number, $10 \mu \mathrm{l}$ of alamarBlue dye (Invitrogen) in $100 \mu 1$ of Panc-1 growth media was added per well. After $4 \mathrm{~h}$, the fluorescent signal was measured using a VIKTOR ${ }^{\mathrm{TM}}$ plate reader (PerkinElmer, MA, USA).

\subsubsection{Quantitative real-time PCR}

To investigate the effect of anti-miR-199a dimeric NCs on hPSC differentiation, hPSCs were seeded in 12-well plates $\left(6 \times 10^{4}\right.$ cells/well $)$ in complete medium. After $18 \mathrm{~h}$, cells were treated with anti-miR-199a dimeric NCs for $24 \mathrm{~h}$, as described previously. Thereafter, cells were activated with TGF- $\beta 1(5 \mathrm{ng} / \mathrm{ml})$ for $24 \mathrm{~h}$. To investigate the effect of anti-miR-199a on its direct target genes, hPSCs were seeded in 12-well plates $\left(6 \times 10^{4}\right.$ cells/well $)$ in complete medium. After 18 $\mathrm{h}$, cells were treated with anti-miR-199a dimeric NCs for $24 \mathrm{~h}$. To study the effect of Panc-1 conditioned medium on PSC differentiation, we collected conditioned medium as described above. hPSCs were seeded in 12-well plates $\left(6 \times 10^{4}\right.$ cells/well $)$ in complete medium. After $18 \mathrm{~h}$, the culture medium was replaced with fresh serum-free medium and incubated for $24 \mathrm{~h}$. Next, Panc-1 conditioned medium was added to the cells for $24 \mathrm{~h}$. To collect Panc-1 conditioned medium, Panc-1 were seeded in 12-well plates at a density of $15 \times$ $10^{4}$ cells/ well. After $24 \mathrm{~h}$, cells were washed and incubated with fresh serumfree medium for $24 \mathrm{~h}$. Subsequently, the medium was collected and frozen at $20^{\circ} \mathrm{C}$. Total RNA was isolated using the GenElute ${ }^{\mathrm{TM}}$ Mammalian Total RNA Miniprep Kit (Sigma) and the RNA amount was measured by a NanoDrop® ND1000 spectrophotometer (Thermo Scientific). Subsequently, cDNA was synthesized with iScript ${ }^{\mathrm{TM}}$ cDNA Synthesis Kit (Bio-Rad, Veenendaal, The Netherlands). A total of $10 \mathrm{ng}$ cDNA was used for each PCR reaction. The realtime PCR primers (Table 1) for Acta-2, Col1 $\alpha 1$, PDGFR- $\beta$, mTOR and RPS18 were purchased from (Sigma). Gene expression levels were normalized to the expression of the house-keeping gene RPS18.

Table 1 Sequences of forward and reverse primers used during real-time PCR

\begin{tabular}{|l|l|l|}
\hline Gene & Forward Primer & Reverse Primer \\
\hline RPS18 & TGAGGTGGAACGTGTGATCA & CCTCTATGGGCCCGAATCTT \\
\hline$\alpha$-SMA & CCCCATCTATGAGGGCTATG & CAGTGGCCATCTCATTTTCA \\
\hline Collagen $1 \alpha 1$ & GTACTGGATTGACCCCAACC & CGCCATACTCGAACTGGAAT \\
\hline PDGF $\beta R$ & AGGCAAGCTGGTCAAGATCT & GCTGTTGAAGATGCTCTCCG \\
\hline
\end{tabular}




\subsubsection{Immunocytochemistry}

To stain for heparan sulfate (HS) on the surface of hPSCs and Panc-1, cells were seeded in 8-well Lab-Tek chamber slides (Thermo Fisher Scientific, NY, USA) $\left(2 \times 10^{4}\right.$ cells/well) in complete medium. After $24 \mathrm{~h}$, cells were washed with PBS and subsequently fixed in PBS containing 4\% formaldehyde (Sigma) for $10 \mathrm{~min}$. After washing with PBS, cell were blocked with PBS containing $0.1 \%(\mathrm{w} / \mathrm{v})$ bovine serum albumin (BSA; VWR, Lutterworth, UK) (PBS/BSA). Afterward, cells were incubated with anti-HS single-chain antibody HS4C3-p5118 (Matrix Biochemistry, Nijmegen, The Netherlands) diluted 1:10 in PBS/BSA for $1 \mathrm{~h}$ at RT. After washing with PBS, the second mouse anti-VSV monoclonal antibody p5D4 (Matrix Biochemistry) diluted 1:10 (PBS/BSA) was incubated in the cells for $1 \mathrm{~h}$ at RT. Next, cells were washed with PBS and incubated with rabbit antimouse polyclonal antibody (Dako, Glostrup, Denmark) diluted 1:100 in PBS/ BSA for $1 \mathrm{~h}$ at RT. After washing with PBS, cells were incubated with Alexa Fluor 594 labeled donkey anti- rabbit (Life Technologies) diluted 1:100 in PBS/BSA for $1 \mathrm{~h}$ at RT. Cells were washed in PBS and nuclei stained with mounting medium containing DAPI (Sigma). Cells were imaged for Alexa 594 (ex. 540/ em. 565) and DAPI (ex. 360/em. 460) using an EVOS FI fluorescent microscope. To investigate the effect of anti-miR-199a on hPSC differentiation, hPSCs were seeded in 24-well plates $\left(2 \times 10^{4}\right.$ cells/well $)$ in complete medium. After $18 \mathrm{~h}$, cells were treated with anti-miR-199a dimeric NCs for $24 \mathrm{~h}$, as described previously. After that cells were activated with TGF- $\beta 1(5 \mathrm{ng} / \mathrm{ml})$ for $48 \mathrm{~h}$. Next, cells were fixed and immunostained for human $\alpha$-smooth muscle actin ( $\alpha$-SMA) and collagen1 (Col1) as described elsewhere ${ }^{31}$.

\subsubsection{Formation of 3D heterospheroids}

hPSCs were plated in 6-well plates and transfected with NC-2 NCs containing anti-miR-199a as described previously. After $24 \mathrm{~h}$, cells were trypsinized and suspended in their respective culture medium at a concentration of $3 \times 10^{5}$ cells $/ \mathrm{ml}$. To evaluate cellular composition of heterospheroids, hPSCs were resuspended in medium containing $1 \mu \mathrm{g} / \mathrm{ml}$ calcein red-orange AM (Life Technologies) and incubated for $1 \mathrm{~h}$ at $37^{\circ} \mathrm{C}$. Panc-1 tumor cells were suspended to the same concentration and afterward mixed 1:1 (vol:vol) with hPSCs. One drop containing $20 \mu \mathrm{l}$ of cell suspension per well was pipetted into the lid of a round bottom suspension culture 96-well plate (Greiner BioOne, Alphen aan den Rijn, The Netherlands). Then the lid was inverted and placed onto a 96-well plate. Wells were filled with $150 \mu$ PBS for humidity. Spheroids were allowed to form for 7 days, imaged under an inverted microscope and their area was 
measured using Image $\mathbf{J}$ software. For sectioning, 3D heterospheroids were collected, embedded in Cryomatrix (Thermo Scientific) and frozen at $-80^{\circ} \mathrm{C}$. Afterward, spheroid were cut into 6 - $\mu$ m-thick sections using a Cryotome FSE (Thermo Scientific) and mounted with fluoroshield ${ }^{\mathrm{TM}}$ with DAPI (Sigma) for staining of nuclei. Sections have been imaged using a Hamamatsu NanoZoomer Digital slide scanner 2.0HT (Hamamatsu Photonics, NJ, USA).

\subsubsection{Immunohistochemical staining of 3D heterospheroids}

The 3D heterospheroids were collected and cut into 6 - $\mu \mathrm{m}$-thick sections as described earlier. Cryosections were fixed in acetone at RT for $15 \mathrm{~min}$ and subsequently rehydrated in PBS for 10 min. Next, sections were incubated with goat-anti collagen type I (Southern BioTech, AL, USA) for $1 \mathrm{~h}$ at RT. Sections were washed with PBS and incubated with secondary Alexa Fluor 594 labeled donkey anti-rabbit diluted 1:100 in PBS containing 5\% human serum. Sections were washed again and mounted with fluoroshield with DAPI (Sigma) for staining of nuclei. Cells were imaged for Alexa 594 (ex. 590/em. 617) and DAPI (ex. 360/em. 460) using a fluorescence microscope (Nikon Eclipse E400, Tokyo, Japan).

\subsubsection{Conditioned medium}

To collect conditioned medium, hPSCs were seeded in 12-well plates at a density of $6 \times 10^{4}$ cells/well. After $24 \mathrm{~h}$, cells were transfected as described previously. After $24 \mathrm{~h}$, cells were incubated with TGF- $\beta 1(5 \mathrm{ng} / \mathrm{ml})$ for $24 \mathrm{~h}$. Afterwards, the cells were washed and incubated with fresh serum-free medium for $24 \mathrm{~h}$. Subsequently the medium was collected and frozen at $-20^{\circ} \mathrm{C}$. To determine the indirect effect of hPSC-conditioned medium on Panc-1 cell growth, Panc-1 were seeded at a density of $2.5 \times 10^{3}$ cells/well in 96-well plates. The next day, conditioned medium was added to the cells. The cell number was monitored 0 , 24 and $48 \mathrm{~h}$ after adding conditioned medium. Cell proliferation was determined using AlamarBlue as described previously.

\subsection{Results}

\subsubsection{Synthesis \& physicochemical properties of NCs}

We prepared several NCs of negatively charged AMOs with positively charged monomeric (NC-1) or dimeric CPPs (NC-2) (Figure 1). Both types of NCs were prepared at various charge ratios (Figure 2). NC-1 and NC-2 showed a tendency to decrease in size with an increase in charge ratio from 1:1 to $10: 1$ (dimeric CPP) or 20:1 (monomeric CPP) (Figure 2C). 
A

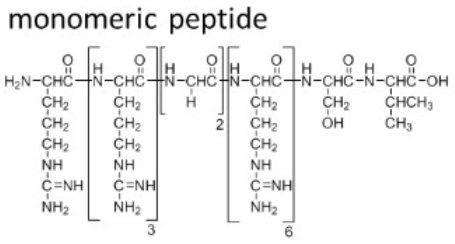

dimeric peptide

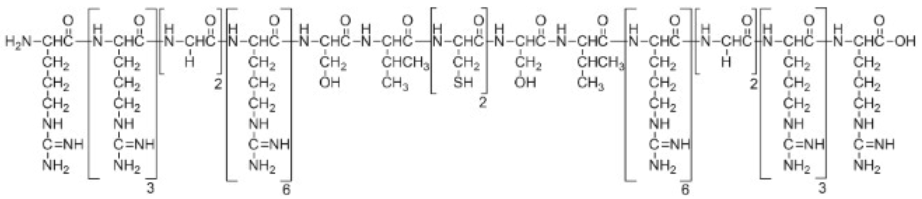

B

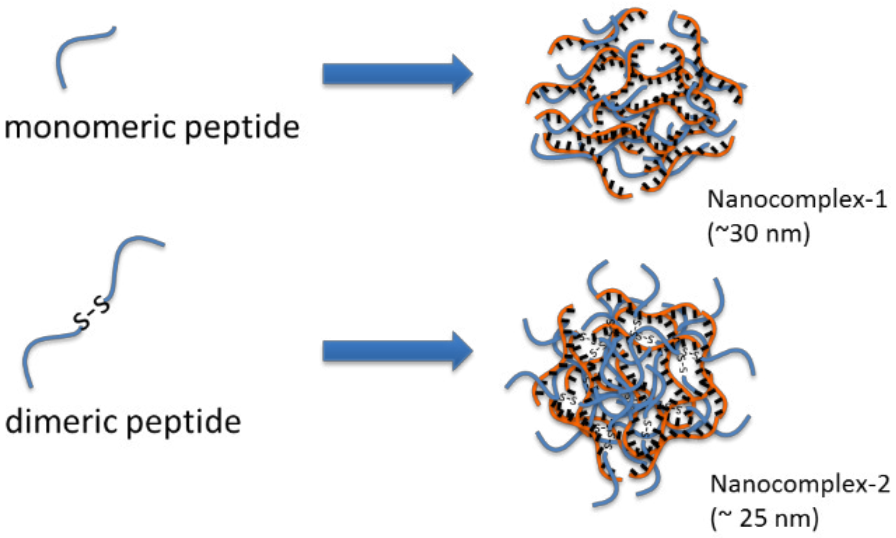

Figure 1. (A) Chemical structures of the monomeric and the dimeric CPP. (B) Schematic representation of $N C-1$ and $N C-2$ nanocomplexes formation. $N C-1$ were prepared from monomeric peptide and AMO, NC-2 from dimeric peptide and AMO. AMO: Anti-miRNA oligonucleotide; CPP: Cell-penetrating peptide; NC: Nanocomplex.

For NC-2, no NCs could be formed above a charge ratio of 10:1 ( \pm ) due to the formation of large aggregates. Incorporation of miRNA into a miR-CPP complex is intended to prevent degradation of miRNA. In spite of identical charge ratios, NC-1 and NC-2 showed significantly distinct surface charges at charge ratio 10:1 (Figure 2D). This gives a strong indication that at the given charge ratio both peptides form structurally different complexes. The surface charge of both NC1 and NC-2 was only slightly affected by changes in charge ratio, ranging between 5 and $20 \mathrm{mV}$ (Figure 2D). NC-1 showed only a slight increase in surface charge at all charge ratios (Figure 2D). In contrast, NC-2 showed a distinct increase in surface charge from ratio 5:1 (6 mV) to $10: 1(18.2 \mathrm{mV})$ (Figure 2D). To investigate the encapsulation of miRNA into NCs, a gel shift assay was performed using electrophoresis. As shown in Figure $2 \mathbf{E} \& \mathbf{F}$, naked miRNA easily passed through the pores of a $2 \%$ agarose gel, while NCs 
remained entrapped at the loading point indicating stable complex formation. In the case of NC-2 (at ratio 1:1), the complexes were not stable as free AMOs moved through the gel (Figure 2F).

A

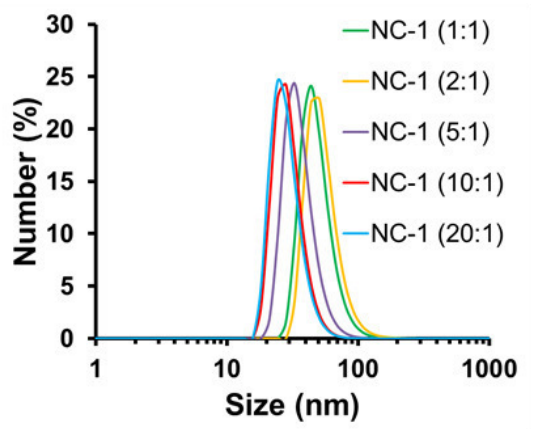

C

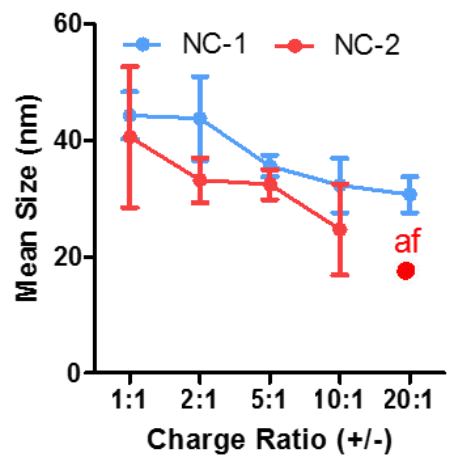

$\mathrm{E}$

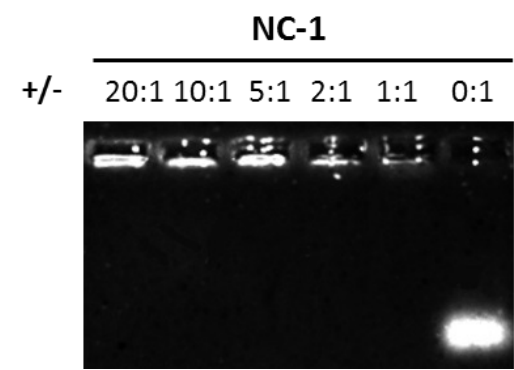

B
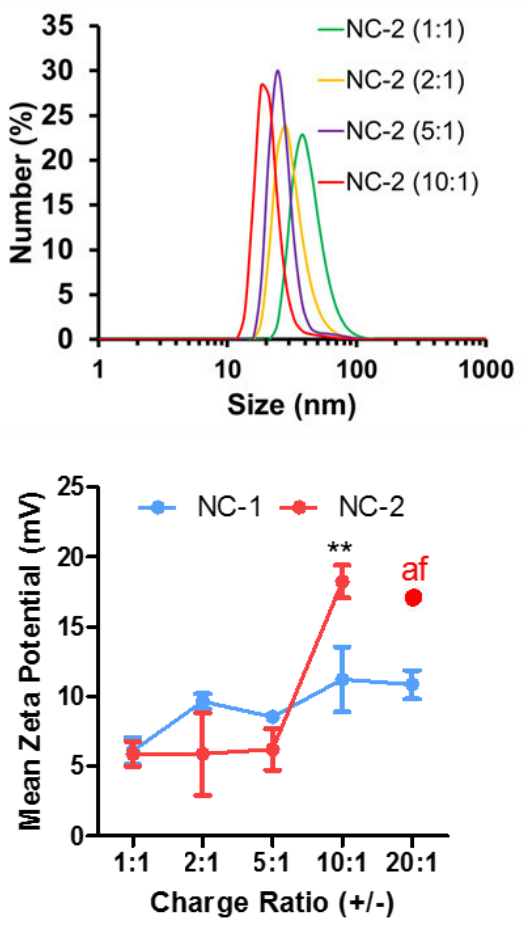

$\mathrm{F}$

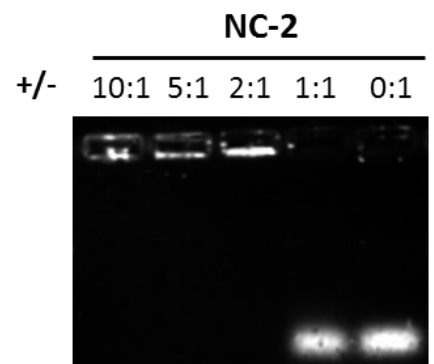

Figure2. Physicochemical characterization of $\mathrm{NC}-1$ and $\mathrm{NC}-2$ nanocomplexes. Representative size distribution of $N C-1$ and $N C$-2 complexes with charge ratios ranging from 1:1 to 20:1 are shown in $(A)$ and $(B)$, respectively. The mean particle size and mean $\zeta$-potential, measured with dynamic light scattering are shown in $(C)$ and $(D)$. Gel shift assay for miRNA encapsulation of $N C-1(E)$ and $N C-2(F)$.Data represent mean \pm standard error of the mean, $n=3$. Statistical analysis was performed by two-tailed unpaired Student's t-test. Statistical differences are **p <0.01. af: Aggregate formation; NC: Nanocomplex. 


\subsubsection{Increased cellular localization \& transfection efficiency by $\mathrm{NC}-2$ compared with $\mathrm{NC}-1$}

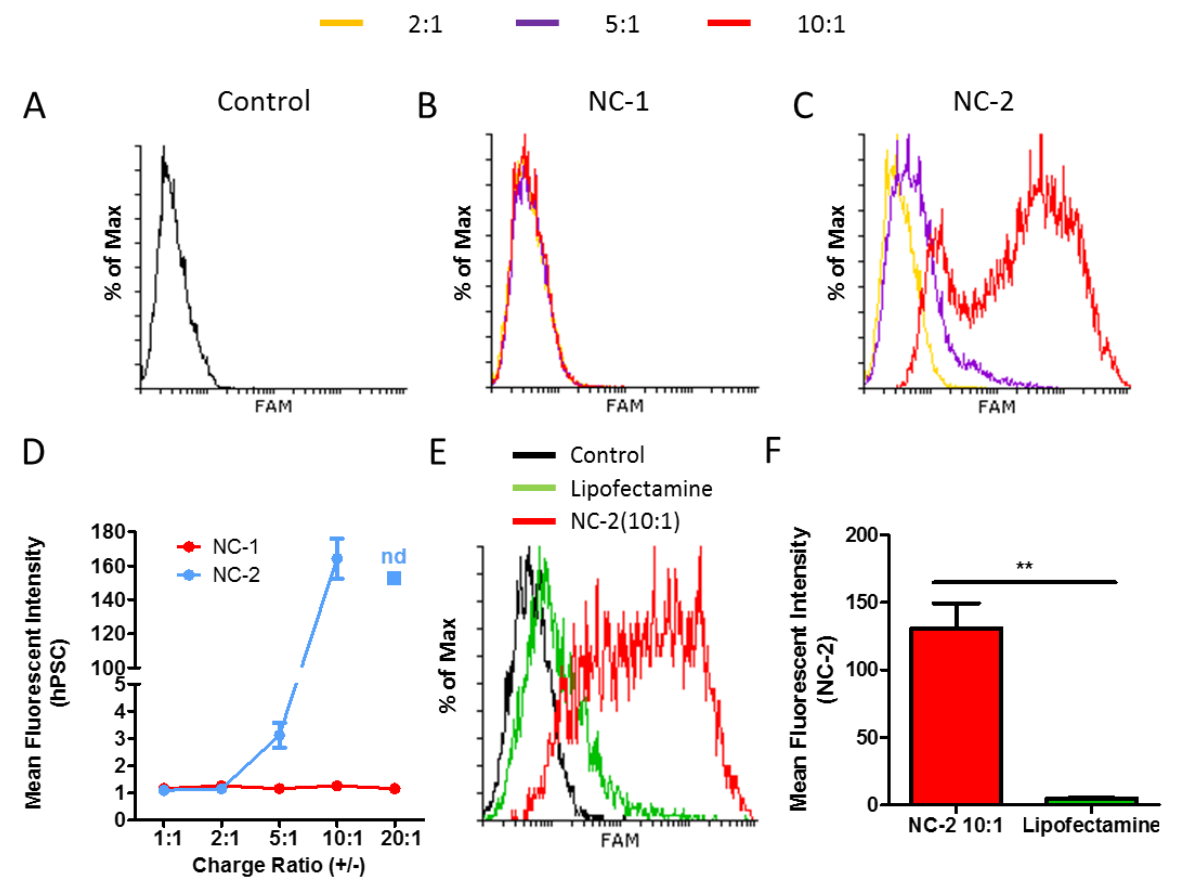

Figure 3. Uptake efficiency of NC-1 and NC-2 nanocomplexes in human pancreatic stellate cells. NCs were formed with monomeric/ dimeric CPP and FAM-labeled AMO. Flow cytometry histograms of hPSCs without transfection (control, [A]) and after exposure to $N C-1(B)$ and $N C-2(C)$ at different charge ratios. Quantitative analyses show the total uptake in hPSCs with $N C-1$ and $N C$-2 at different charge ratios (D). Histogram showing a comparison between uptake for lipofectamine-2000 complexes and NC-2 nanocomplexes at charge ratio 10:1 (E). Quantitative analysis of the total uptake of lipofectamine-2000 complexes and NC-2 nanocomplexes at charge ratio 10:1 (F). Statistical analysis was performed by two-tailed unpaired Student's t-test. Data represent mean \pm standard error of the mean, $n=3$. Statistical differences are $* * p<0.01$. AMO: Anti-miRNA oligonucleotide; CPP: Cell-penetrating peptide; hPSC: Human pancreatic stellate cell; NC: Nanocomplex; nd: Not determined.

To investigate the uptake efficiency of the formed NCs, we performed flow cytometry for all NCs in hPSCs (Figure 3). NC-1 NCs at any charge ratio showed very low to no uptake in hPSCs (Figure 3B \& D). Intriguingly, NC-2 NCs showed a remarkably increased uptake from ratios 5:1 to 10:1 (Figure 3C \& D). Compared with NC-1, NC-2 had about 130-fold higher uptake by hPSCs at charge ratio 10:1 (Figure 3D). In addition, the delivery efficiency with NC-2 
(10:1) was significantly higher in hPSCs compared with miRNA uptake with the gold standard transfection reagent lipofectamine-2000 (Figure 3E \& F).

A

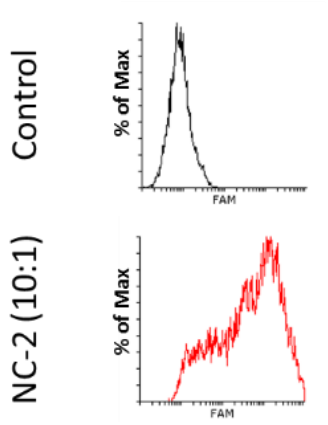

F

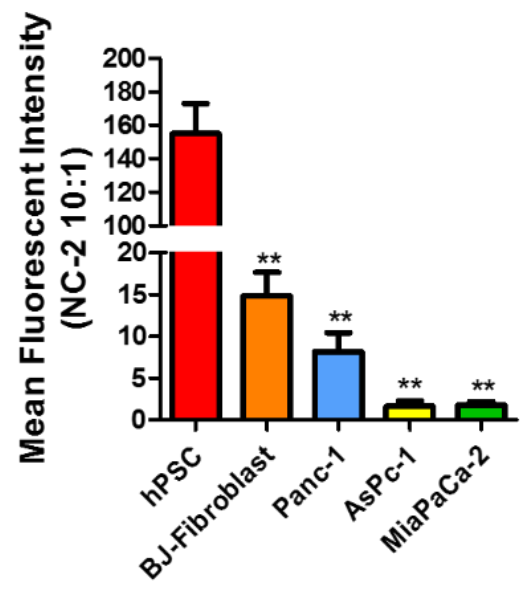

B
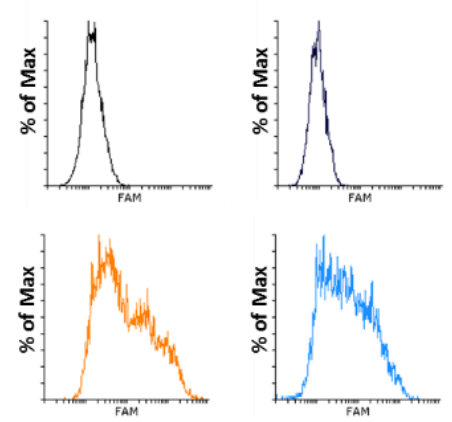

G
D

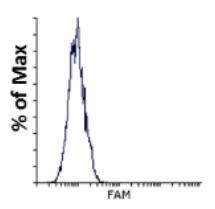

E
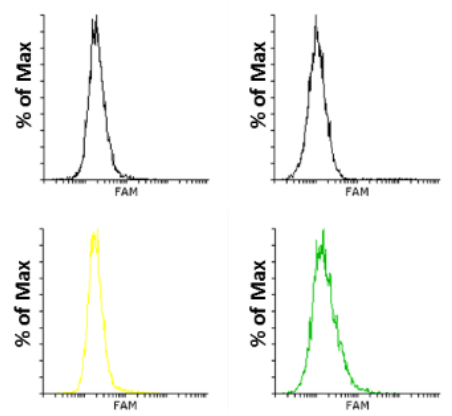

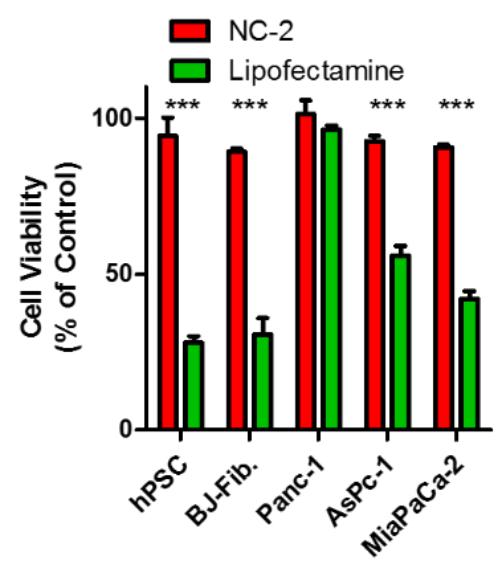

Figure 4. Uptake efficiency of NC-2 (10:1) nanocomplexes in human pancreatic stellate cells after $6 \boldsymbol{h}$ of incubation. NC-2 were formed with dimeric CPP and FAMlabeled AMO. Histograms showing flow cytometric analyses of hPSCs (A), BJfibroblasts (B), Panc-1 (C), AsPc-1 (D) and MiaPaCa-2 (E) without transfection (control) and after exposure to NC-2 at the charge ratio 10:1. (F) Quantitative analyses show the total uptake in hPSCs, BJ-fibroblasts, Panc-1, AsPc-1 and MiaPaCa-2 with $N C$-2 at charge ratio 10:1 (colors match with histograms $[A-E])$. (G) Cell viability with NC-2 (10:1) and lipofectamine-2000 in hPSCs, BJ-fibroblasts, Panc-1, AsPc-1 and MiaPaCa-2 after 24 h of incubation. Statistical analysis was performed by two-tailed unpaired Student's t-test. Data represent mean \pm standard error of the mean, $n=3$. Statistical differences are ***p <0.001. AMO: Anti-miRNA oligonucleotide; CPP: Cellpenetrating peptide; hPSC: Human pancreatic stellate cell; NC: Nanocomplex. 
In addition, the uptake of NC-2 $(10: 1)$ was significantly higher $(\mathrm{p}<0.01)$ in hPSCs compared with BJ-fibroblasts and pancreatic tumor cells (i.e., Panc-1, AsPc-1 and MiaPaCa-2) (Figure 4A-F).Furthermore, we visualized the uptake of NC-2 in these cells using fluorescence microscopy, confirming that NC-2 NCs had much higher localization in hPSCs compared with fibroblasts (BJfibroblasts) and pancreatic tumor cell lines, as indicated by arrows (Figure 5). During their incubation, we found no cell death as confirmed by AlamarBlue assay, while lipofectamine-2000 showed severe toxicity in all cell types except Panc-1 (Figure 4G). Particularly, hPSCs and fibroblasts had the maximal toxicity ( $\sim 75 \%$ reduction in viability) with lipofectamine-2000.

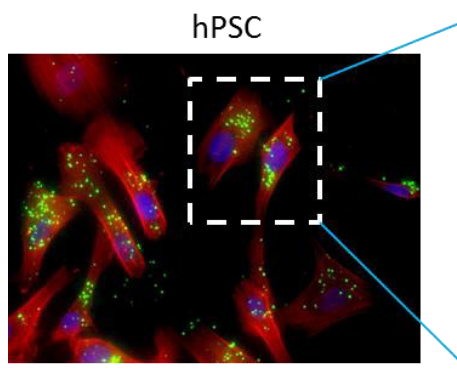

Panc-1

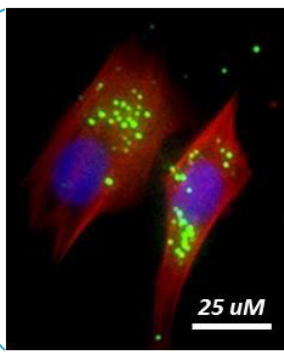

MiaPaCa-2

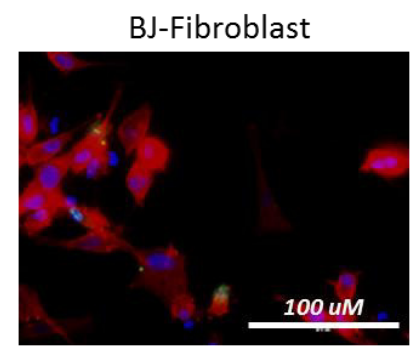

AsPc-1
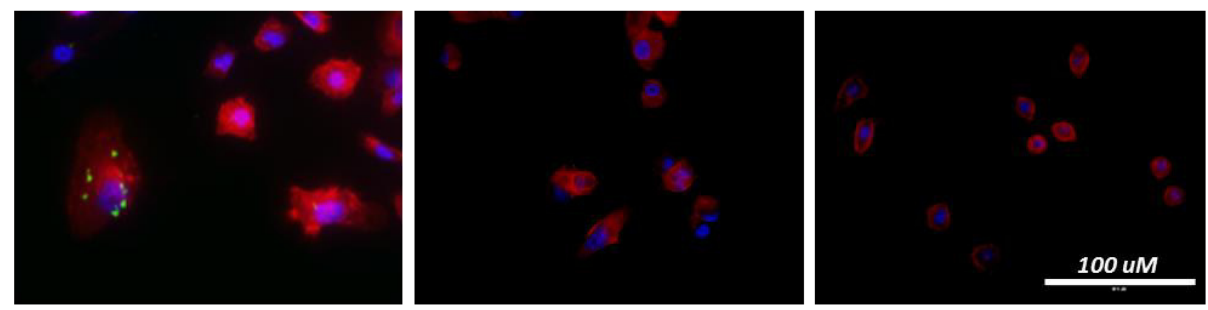

Figure 5. Fluorescent microscopic images of the cellular uptake of NC-2 nanocomplexes formed with FAM-labeled anti-miRNA oligonucleotide at charge ratio 10:1. Pictures were taken after 6-h incubation with hPSCs, BJ-fibroblasts, Panc-1, AsPc-1 and MiaPaCa-2. Nuclei are stained with DAPI (blue), filamentous actin with phalloidin (red). AMOs were labeled with FAM (green). Scale bar: $100 \mu \mathrm{m}$. AMO: AntimiRNA oligonucleotide; CPP: Cell-penetrating peptide; hPSC: Human pancreatic stellate cell; NC: Nanocomplex. 


\subsubsection{NC-2 are partly taken up by clathrin-mediated endocytosis}

A

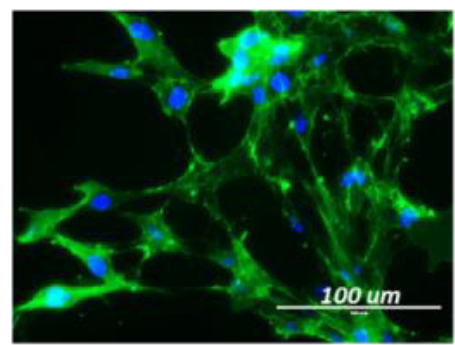

C

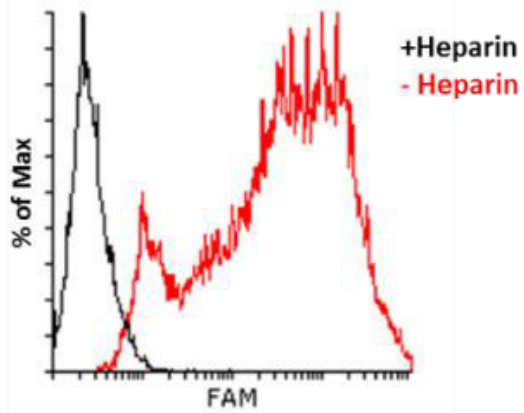

B

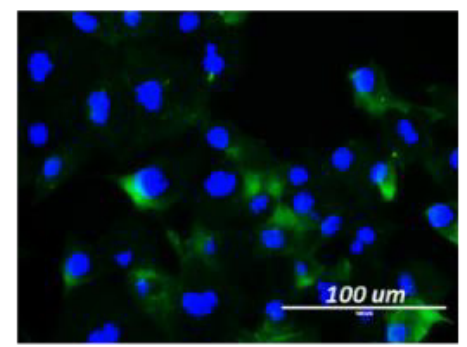

D

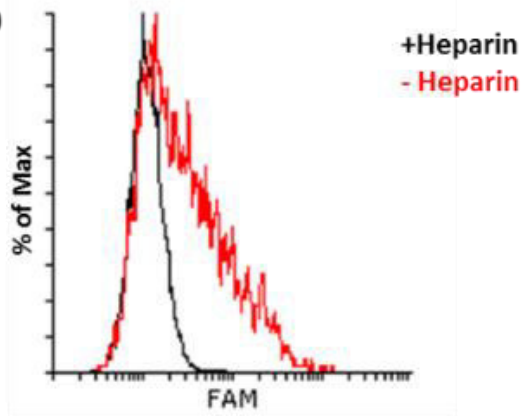

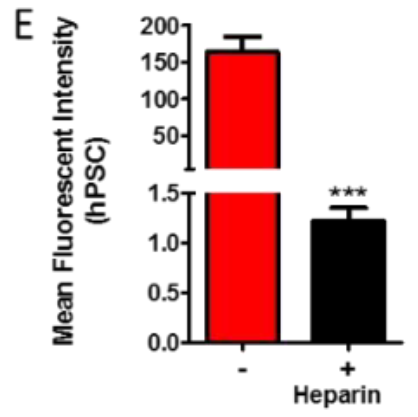
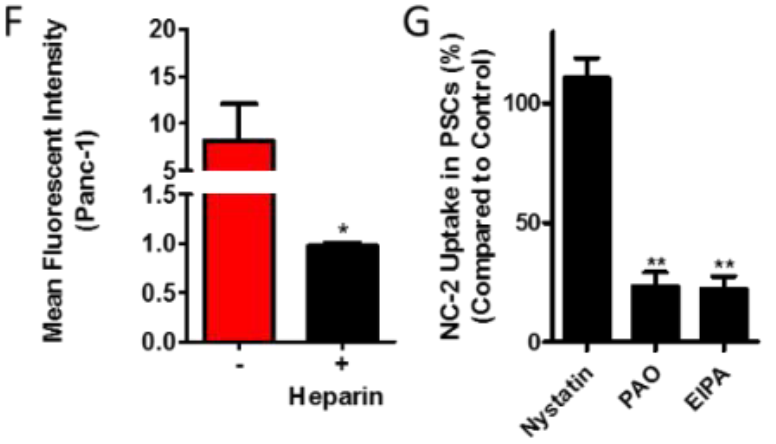

Figure 6. Role of Heparan sulfate proteoglycans in uptake of NC-2 Nanocomplexes. Presence of HSPGs on the surface of hPSCs (A) and Panc-1 (B). Scale bar: $100 \mu \mathrm{m}$. Histogram of hPSCs $(C)$ and Panc-1 (D) cells after $6 \mathrm{~h}$ incubation with NC-2 (10:1) Nanocomplexes in the absence and presence of heparin preincubated with the cells 30 min before addition of NC-2. Quantitative analyses for the effect of heparin on the NC2 transfection efficiency in hPSCs (E) and Panc-1 (F), respectively. Endocytosis mechanism for the uptake of NC-2 by hPSCs $(G)$. Uptake of Nanocomplexes by hPSCs $s$ after 6 h incubation with $\mathrm{NC}-2$ (10:1) Nanocomplexes incubated with nystatin, PAO and EIPA for 10 mins prior to the incubation with $N C-2(G)$. Statistical analysis was performed by two-tailed unpaired student t-test. Data represent mean \pm SEM., $n=3$. Statistical differences are $* p<0.05, * * p<0.01$ and $* * * p<0.001$. EIPA: $5-(N-$ ethyl- $N$ - 
isopropyl)-amiloride; hPSC: Human pancreatic stellate cell; HSPG: Heparan sulfate proteoglycan; NC: Nanocomplex.

Evidence exists showing that lipo-and polyplexes initiate endocytosis via the attachment to HS proteoglycans (HSPGs) ${ }^{32,33}$. Fluorescent staining for heparan sulfate (HS) on the surface of hPSCs and Panc-1 cancer cells showed an increased expression of HSs on the surface of hPSCs compared with Panc-1 (Figure 6A \& B). To prove an HSPG-mediated uptake mechanism, we performed a competition assay in which heparan was added to the culture medium to compete with HSPG for the binding of NCs. The competition assay resulted in the complete inhibition of NC-2 uptake by hPSCs and Panc-1 cells (Figure 6C-F). These data indicate that HSPGs are, at least partially, involved in the internalization of the NC-2 NCs by hPSCs and Panc-1. To study the mechanism of endocytosis by which the NC-2 NCs are taken up into hPSCs, we incubated hPSCs with known inhibitors for clathrin-mediated endocytosis, phenylarsine oxide ${ }^{34}$, macropinocytosis, 5-(N-ethyl-N-isopropyl)-amiloride $(\mathrm{EIPA})^{35}$, and for caveolin-dependent endocytosis, nystatin ${ }^{36}$. We found that nystatin did not affect the uptake of NC-2 by hPSCs showing that caveolindependent endocytosis does not play a role in their uptake (Figure 6G). On the other hand, treatment of hPSCs with PAO and EIPA did significantly block the uptake of NC-2 by hPSCs (Figure 6G).

\subsubsection{NC-2 mediated anti-miRNA-199a delivery inhibits hPSC activation into CAFs}

We synthesized anti-miR-199a oligonucleotides with 2'-O-methyl modification with phosphodiester backbone and then prepared NCs with dimeric CPPs. Using NC-2/anti-miR199a, we were able to significantly induce mTOR in hPSCs (Supplementary Figure 1), and one of the target genes of miR-199a, which was previously introduced using ingenuity pathway analysis (IPA) ${ }^{37}$. To differentiate hPSCs into their activated state, we incubated them with human recombinant TGF- $\beta 1$, resulting in the upregulation of the hPSC activation transcription markers Acta2, Col1a1 and PDGFR- $\beta$ (Figure 7). Interestingly, incubation of NC-2/anti-miR199a with hPSCs resulted in a significant inhibition of Acta2, Col1a1 and PDGFR- $\beta$ gene expression compared with the nontransfected control and scrambled AMO control (Figure 7A-C). Further- more, we examined the 
effect on the protein expression of $\alpha$-SMA and Coll and found a clear downregulation of these markers with NC-2/anti-miR199a (Figure 7D).

A

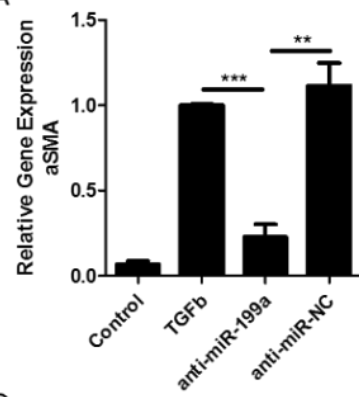

D
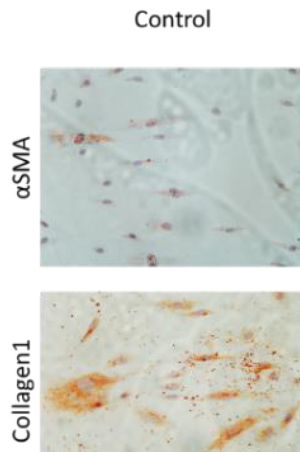

B

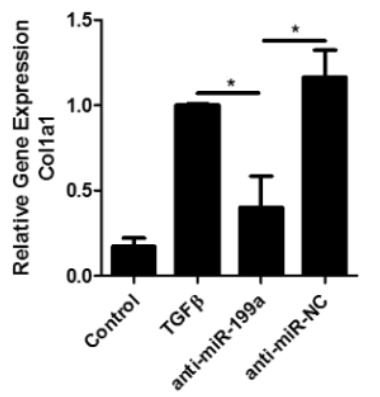

TGFB
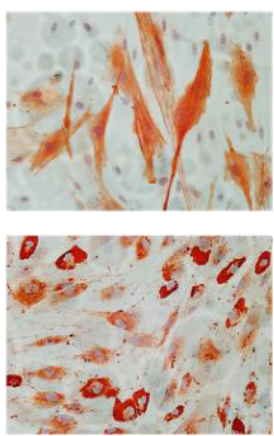

TGF $\beta+$
C

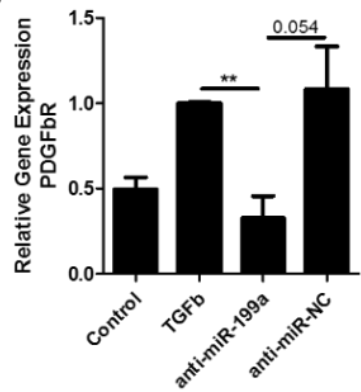

TGF $\beta+$ $\mathrm{NC}-2$ (miR-NC)
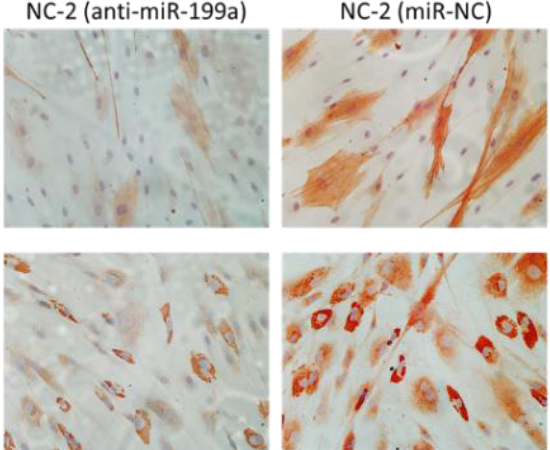

Figure 7. Nanocomplexes of anti-miR-199a oligonucleotide and their inhibitory effects on human pancreatic stellate cells. Gene expression of Acta-2 (A), Collal (B) and PDGFR- $\beta(C)$ relative to house-keeping gene RPS18 in hPSCs, activated with TGF $\beta$, after transfection with anti-miR-199a. (D) Protein expression of $\alpha-S M A$ and Coll after transfection with anti-miR-199a containing nanocomplexes. Statistical analysis was performed by two-tailed unpaired Student's t-test. Data represent mean \pm standard error of the mean, $n=3$. Statistical differences are $* p<0.05 ; * * p<0.01 . \alpha-S M A$ : Alphasmooth muscle actin; Collal: Collagen1al; hPSC: Human-derived pancreatic stellate cell; NC: Nanocomplex.

\subsection{5 hPSC treatment with NC-2/anti-miR199a inhibits the}

\section{formation of $3 \mathrm{D}$ heterospheroids}

Furthermore, to study the impact of anti-miR199a on hPSC-mediated tumor formation, we established a stroma-rich 3D spheroid assay. Using the hanging drop method, we generated heterospheroids consisting of Panc-1 and hPSCs (Figure 8A). We found that heterospheroids composed of hPSCs and Panc-1 expressed high amounts of collagen-1 when compared with Panc-1 spheroids (Figure 8A). To get an idea about the paracrine effects which Panc-1 exert on 
hPSCs within the heterospheroids, we performed a Panc-1 conditioned medium experiment. We showed that conditioned medium collected from Panc-1 could induce hPSC differentiation as shown by the significant increase of the hPSC activation markers Acta-2 (Figure 8B), Colla1 (Figure 8C) and PDGFR- $\beta$ (Figure 8D).
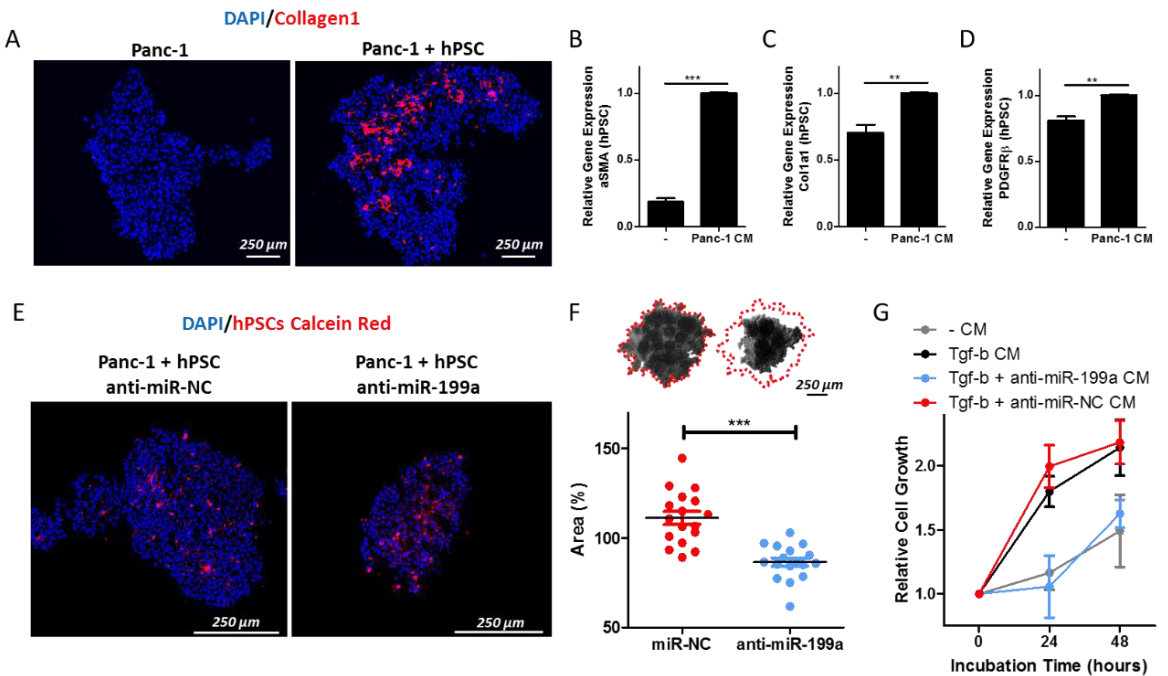

Figure 8. Effect of anti-mir-199a in tumor spheroids. (A) Images made from 6 $\mu$ m thick heterospheroid sections stained for Collagen1. Nuclei are stained with Dapi, Collagen1 was stained with Alexa 594. Scale bar $=250 \mathrm{um}$. Gene expression of $\alpha \mathrm{SMA}(B)$, Collal $(C)$ and PDGF $\beta R(D)$ relative to house-keeping gene RPS18 in hPSCs, activated with Panc-1 conditioned medium. (E) Presence Calein red labeled hPSCs in 6um Panc1/PSC heterospheroid sections. Spheroids were transfected with Nanocomplexes containing anti-miR-199a or anti-miR-NC. Scale bar $=250$ um. Nuclei are stained with Dapi, hPSCs were stained with calcein red-orange AM. $(F)$ Nanocomplexes containing anti-miR-199a oligonucleotide and their inhibitory effect on the formation of heterospheroids composed of Panc-1 and hPSCs. (C) Representative pictures of heterospheroids $(n=17)$ formed with Panc-1 and hPSCs transfected with scrambled miR and anti-miR-199a. (F) Dotted line represents the contour of the spheroid formed with miR-NC treated hPSCs. Scale bar $=250 \mathrm{um}$. $(G)$ Effect of conditioned medium of antimiR-199a treated hPSCs on Panc-1 cell growth over 48 hours. Statistical analysis was performed by two-tailed unpaired student t-test. Data represent mean $\pm S E M$., $n=3$. Statistical differences are $* * * p<0.005$.

To evaluate if NCs carrying anti-miR199a could inhibit the growth of 3D heterospheroids composed of hPSCs and Panc-1 tumor cells, we transfected hPSCs with NC-2/anti-miR199a and used them for spheroid formation. After 7 days, we found that heterospheroids composed of Panc-1 and hPSCs transfected 
with anti-miR-199a formed smaller spheroids compared with the heterospheroids containing hPSCs transfected with scrambled miRNA (Figure 8F). To prove that the smaller spheroids do not consist of cancer cells only, we have labeled hPSCs transfected with NC-2/anti-miR199a with Calcein red AM prior to spheroids formation and still found these cells within the spheroids after 7 days (Figure 8E). Interestingly, there were similar number of hPSCs in antimiR-199a transfected spheroids compared with the control spheroids. In contrast, the number of tumor cells was much lesser in the spheroids formed with anti-mir199a-hPSCs compared with the control spheroids (Figure 8E). To get an indication about the paracrine effects which hPSCs exert on Panc-1 cell growth, we performed a conditioned medium experiment (Figure 8G). We showed that conditioned medium collected from the activated hPSCs stimulated the growth of Panc-1 tumor cells compared with conditioned medium from normal hPSCs (Figure 8G). When hPSCs were transfected with anti-miR199a, the cell growth of Panc-1 tumor cells was inhibited (Figure 8G).

\subsection{Discussion}

In this study, we have developed a novel strategy to deliver AMO to hPSCs, the key precursor cells of CAFs in pancreatic cancer. A dimeric form of an argininerich CPP was synthesized and used for preparing NCs with AMOs. Intriguingly, these NCs showed very high uptake by hPSCs compared with the NCs with the monomeric forms. Although branching or multivariant approaches may enhance CPP-mediated uptake, our dimeric CPP-based NCs were highly specific to hPSCs compared with normal fibroblasts or pancreatic tumor cells. To this end, we examined different mechanisms for uptake by hPSCs. Then, we applied these NCs for specific delivery of an AMO against miR-199a (induced in activated hPSCs and $\mathrm{CAFs}^{37}$ ) to hPSCs in 2D monocultures and 3D co-cultures. Interestingly, delivery of anti-miR-199a into hPSCs inhibited their differentiation into myofibroblasts and hPSC-mediated tumor cell growth.

Evidence suggests that miRNAs are key endogenous regulators of differentiation of $\mathrm{CAF}$ and their functions in the tumor microenvironment ${ }^{4}$. Reversal of upregulated or downregulated miRNA using anti-miRNA or miRNA-mimics, respectively, seems to be a highly interesting approach to inhibit CAF-mediated tumor-supporting effects ${ }^{4}$. However, a major challenge is the specific delivery of these single stranded anti-miRNA or miRNA-mimic oligonucleotides into the target cells ${ }^{5}$. In this study, we designed a dimeric form of an arginine- rich CPP using a disulfide bridge to attain a longer and more flexible chain which allows 
for a better interaction with AMO. In addition, these complexes at charge ratio 10:1 have a higher availability of CPP at the surface of the NCs to achieve better interactions with the cellular surface for their efficient uptake. Furthermore, NC2 possessed a higher surface charge compared with NC-1 at charge ratio 10:1 which suggests that more CPPs are available on the surface of NC-2.

The cellular uptake study in hPSCs showed that dimeric CPP-based NC-2 were 130-fold higher taken up by hPSCs compared with monomeric CPP-based NC1 (Figure 3). These data clearly showed the benefit of our dimeric form of CPP over the monomeric form. Although studies have shown that branching or multimeric forms of CPP may enhance their cellular uptake ${ }^{38,39}$, in the present study we surprisingly found that NC-2 complexes were not only enhancing general cellular uptake but also very specifically enhanced cellular uptake in hPSCs compared with normal fibroblasts and different pancreatic tumor cells (Panc-1, MiaPaCa-2 and AsPc-1) (Figures 4 \& 5). This preferential uptake by hPSCs led us to explore this observation further. Fluorescent staining revealed increased expression of HSs on the surface of hPSCs compared with Panc-1 cancer cells (Figure 6A \& B). Competition studies with heparan indicate that NC-2 are taken up by an HSPG-dependent mechanism (Figure 6C-F). In the case of arginine-rich CPPs, it has been proposed that the interaction of peptides with membrane-associated proteoglycans triggers the induction of actin organization and macropinocytosis ${ }^{40}$. The affinity for the interaction with HSPG seems to be dependent on the structure of arginine-rich CPPs ${ }^{40}$. Additionally, it has been found that arginine-rich CPPs bind to HSPGs with binding constants of nanomolar concentration and therefore can be considered as receptors for intracellular uptake ${ }^{32,41-43}$. Blocking of NC-2 uptake with PAO and EIPA did significantly block the uptake of NC-2, indicating that next to macropinocytosis, clathrin-mediated endocytosis plays a role in the uptake of NCs by hPSCs (Figure 6G). The receptor (clathrin) mediated uptake of NCs can, at least up to some extent, explain the preferential cellular uptake of NC-2 NCs by hPSCs compared with BJ-fibroblasts, Panc-1, MiaPaCa-2 and AsPc-1 cells. Likely, this is due to the NCs interacting with HSPGs attached to trans- membrane receptors on the cellular surface of hPSCs.

Recently, miRNA-199a was identified as a novel therapeutic target in pancreatic tumor stromal fibroblasts in patients ${ }^{37}$. It was shown, that miRNA-199a was induced in patient-derived CAFs and activated hPSCs and regulated the differentiation of these cells as well as hPSC-induced protumorigenic effects ${ }^{37}$. In this study, we showed that NC-2 containing active AMOs against miRNA- 
199a could inhibit hPSC differentiation at both mRNA and protein level, as shown with the CAF markers $\alpha$-SMA, Col-1a1 and PDGFR- $\beta$. In addition, we demonstrated that delivery of anti-miR-199a leads to activation of the direct target gene mTOR in hPSCs (Supplementary Figure 1), implying that the effects observed on hPSCs are miR-199a related. These data clearly demonstrate the feasibility of our approach enabling the transfection of an active AMO into hPSCs, leading to the inhibition of hPSC activation markers.

Within the tumor microenvironment, activated hPSCs and CAFs stimulate tumor cell growth by secreting cytokines and thereby induce tumor progression. Our 3D heterospheroidal system mimics the interaction between tumor cells and hPSCs, as shown with the presence of Col1 staining as marker for activated hPSCs (Figure 8A). Our conditioned medium studies also confirmed that hPSCs get activated by factors secreted by Panc-1 (Figure 8B-D). The inhibition of the growth of 3D heterospheroids, composed of hPSC and Panc-1 tumor cells, after anti-miR-199a delivery into hPSCs clearly demonstrated that inhibition of antimiR-199a using our novel NC-2 is capable of intervening into hPSC-induced tumor-inducing effects. A previous study has shown that transfection of antimiR-199a may reduce the hPSCs growth ${ }^{37}$. From the fluorescent images of spheroids (Figure 8E), it is apparent that only number of tumor cells was reduced in spheroids formed with anti-miR-199a hPSCs compared with control spheroids. To better understand whether the 3D spheroid growth inhibitory effects of the targeted anti-miR199a were due to the inhibition of hPSC-mediated Panc-1 growth but not majorly due to hPSC growth itself, we performed additional experiments using hPSC conditioned medium. We found that conditioned medium obtained from TGF $\beta$-activated hPSCs induces Panc-1 tumor cell growth, while the activated hPSCs treated with anti-miR-199a did not induce the growth of Panc-1 tumor cells, confirming that delivery of anti-miR199a indeed inhibits hPSC-mediated tumor cell growth both in 2D monocultures and 3D co-cultures.

\subsection{Conclusion}

In conclusion, we have developed a novel dimeric CPP-based self-assembling delivery system to specifically deliver AMOs to hPSCs compared with tumor cells. We also demonstrated that our miRNA delivery system is functional in delivering active miRNA to hPSCs and in inhibiting their differentiation into tumor-promoting CAF phenotype. Altogether, this study presents a highly 
efficient peptide-based anti-miRNA delivery system to hPSCs to inhibit hPSCmediated protumorigenic activity.

\subsection{Financial supports}

The study was supported by the Swedish Research Council, Stockholm (project no. 2011-5389) and the MIRA Institue for Biomedical Technology and Technical Medicine, University of Twente, The Netherlands

\subsection{References}

1 Zhang, Y., Wang, Z. \& Gemeinhart, R. A. Progress in microRNA delivery. $J$ Control Release 172, 962-974, doi:10.1016/j.jconrel.2013.09.015 (2013).

2 Calin, G. A. \& Croce, C. M. MicroRNA signatures in human cancers. Nature reviews. Cancer 6, 857-866, doi:10.1038/nrc1997 (2006).

3 Lin, S. \& Gregory, R. I. MicroRNA biogenesis pathways in cancer. Nature reviews. Cancer 15, 321-333, doi:10.1038/nrc3932 (2015).

4 Kuninty, P. R., Schnittert, J., Storm, G. \& Prakash, J. MicroRNA Targeting to Modulate Tumor Microenvironment. Front Oncol 6, 3, doi:10.3389/fonc.2016.00003 (2016).

5 Li, Z. \& Rana, T. M. Therapeutic targeting of microRNAs: current status and future challenges. Nature reviews. Drug discovery 13, 622-638, doi:10.1038/nrd4359 (2014).

6 Geary, R. S., Norris, D., Yu, R. \& Bennett, C. F. Pharmacokinetics, biodistribution and cell uptake of antisense oligonucleotides. Adv Drug Deliv Rev 87, 46-51, doi:10.1016/j.addr.2015.01.008 (2015).

7 Pereira, D. M., Rodrigues, P. M., Borralho, P. M. \& Rodrigues, C. M. Delivering the promise of miRNA cancer therapeutics. Drug discovery today 18, 282-289, doi:10.1016/j.drudis.2012.10.002 (2013).

8 Broderick, J. A. \& Zamore, P. D. MicroRNA therapeutics. Gene therapy 18, 1104-1110, doi:10.1038/gt.2011.50 (2011).

9 Kortylewski, M. \& Nechaev, S. How to train your dragon: targeted delivery of microRNA to cancer cells in vivo. Molecular therapy : the journal of the American Society of Gene Therapy 22, 1070-1071, doi:10.1038/mt.2014.73 (2014).

10 Wang, H. et al. Recent progress in microRNA delivery for cancer therapy by non-viral synthetic vectors. Adv Drug Deliv Rev 81, 142-160, doi:10.1016/j.addr.2014.10.031 (2015).

$11 \mathrm{He}, \mathrm{H}$. et al. Low molecular weight protamine (LMWP): a nontoxic protamine substitute and an effective cell-penetrating peptide. Journal of controlled release : official journal of the Controlled Release Society 193, 63-73, doi:10.1016/j.jconrel.2014.05.056 (2014). 
van Asbeck, A. H. et al. Molecular parameters of siRNA--cell penetrating peptide nanocomplexes for efficient cellular delivery. ACS Nano 7, 3797-3807, doi:10.1021/nn305754c (2013).

13 Lee, S. H., Castagner, B. \& Leroux, J. C. Is there a future for cellpenetrating peptides in oligonucleotide delivery? Eur J Pharm Biopharm 85, 5-11, doi:10.1016/j.ejpb.2013.03.021 (2013).

14 Brock, R. The uptake of arginine-rich cell-penetrating peptides: putting the puzzle together. Bioconjug Chem 25, 863-868, doi:10.1021/bc500017t (2014).

15 Said Hassane, F., Saleh, A. F., Abes, R., Gait, M. J. \& Lebleu, B. Cell penetrating peptides: overview and applications to the delivery of oligonucleotides. Cell Mol Life Sci 67, 715-726, doi:10.1007/s00018009-0186-0 (2010).

16 Margus, H., Padari, K. \& Pooga, M. Cell-penetrating peptides as versatile vehicles for oligonucleotide delivery. Mol Ther 20, 525-533, doi:10.1038/mt.2011.284 (2012).

17 Sicard, F., Gayral, M., Lulka, H., Buscail, L. \& Cordelier, P. Targeting miR-21 for the therapy of pancreatic cancer. Mol Ther 21, 986-994, doi:10.1038/mt.2013.35 (2013).

18 Brand, R. E. \& Tempero, M. A. Pancreatic cancer. Curr Opin Oncol 10, 362-366 (1998).

19 Hidalgo, M. Pancreatic cancer. The New England journal of medicine 362, 1605-1617, doi:10.1056/NEJMra0901557 (2010).

20 Jemal, A. et al. Cancer statistics, 2006. CA: a cancer journal for clinicians 56, 106-130 (2006).

21 Apte, M. V., Wilson, J. S., Lugea, A. \& Pandol, S. J. A starring role for stellate cells in the pancreatic cancer microenvironment. Gastroenterology 144, 1210-1219, doi:10.1053/j.gastro.2012.11.037 (2013).

22 Apte, M. V. et al. Desmoplastic reaction in pancreatic cancer: role of pancreatic stellate cells. Pancreas 29, 179-187 (2004).

23 Rasheed, Z. A., Matsui, W. \& Maitra, A. in Pancreatic Cancer and Tumor Microenvironment (eds P. J. Grippo \& H. G. Munshi) (2012).

24 Masamune, A. et al. Alteration of the microRNA expression profile during the activation of pancreatic stellate cells. Scandinavian journal of gastroenterology 49, 323-331, doi:10.3109/00365521.2013.876447 (2014).

25 Masamune, A. \& Shimosegawa, T. Pancreatic stellate cells--multifunctional cells in the pancreas. Pancreatology 13, 102-105, doi:10.1016/j.pan.2012.12.058 (2013).

26 Jesnowski, R. et al. Immortalization of pancreatic stellate cells as an in vitro model of pancreatic fibrosis: deactivation is induced by matrigel and N-acetylcysteine. Lab Invest 85, 1276-1291, doi:10.1038/labinvest.3700329 (2005). 
27 Rachagani, S. et al. Clinical implications of miRNAs in the pathogenesis, diagnosis and therapy of pancreatic cancer. Advanced drug delivery reviews 81, 16-33, doi:10.1016/j.addr.2014.10.020 (2015).

28 Ali, S. et al. Contribution of microRNAs in understanding the pancreatic tumor microenvironment involving cancer associated stellate and fibroblast cells. Am J Cancer Res 5, 1251-1264 (2015).

29 Aubry, S. et al. Cell-surface thiols affect cell entry of disulfideconjugated peptides. FASEB journal : official publication of the Federation of American Societies for Experimental Biology 23, 29562967, doi:10.1096/fj.08-127563 (2009).

30 Erazo-Oliveras, A. et al. Protein delivery into live cells by incubation with an endosomolytic agent. Nature methods 11, 861-867, doi:10.1038/nmeth.2998 (2014).

31 Bansal, R. et al. PEGylation improves pharmacokinetic profile, liver uptake and efficacy of Interferon gamma in liver fibrosis. $J$ Control Release 154, 233-240, doi:10.1016/j.jconrel.2011.05.027 (2011).

32 Favretto, M. E., Wallbrecher, R., Schmidt, S., van de Putte, R. \& Brock, R. Glycosaminoglycans in the cellular uptake of drug delivery vectors bystanders or active players? Journal of controlled release : official journal of the Controlled Release Society 180, 81-90, doi:10.1016/j.jconrel.2014.02.011 (2014).

33 Ruponen, M. et al. Extracellular glycosaminoglycans modify cellular trafficking of lipoplexes and polyplexes. J Biol Chem 276, 33875-33880, doi:10.1074/jbc.M011553200 (2001).

34 Gibson, A. E., Noel, R. J., Herlihy, J. T. \& Ward, W. F. Phenylarsine oxide inhibition of endocytosis: effects on asialofetuin internalization. Am J Physiol 257, C182-184 (1989).

35 Meier, O. et al. Adenovirus triggers macropinocytosis and endosomal leakage together with its clathrin-mediated uptake. J Cell Biol 158, 1119-1131, doi:10.1083/jcb.200112067 (2002).

36 Rothberg, K. G. et al. Caveolin, a protein component of caveolae membrane coats. Cell 68, 673-682 (1992).

37 Kuninty, P. R. et al. MicroRNA-199a and -214 as potential therapeutic targets in pancreatic stellate cells in pancreatic tumor. Oncotarget 7, 16396-16408, doi:10.18632/oncotarget.7651 (2016).

38 Monreal, I. A. et al. Branched dimerization of Tat peptide improves permeability to HeLa and hippocampal neuronal cells. Chem Commun (Camb) 51, 5463-5466, doi:10.1039/c5cc00882d (2015).

39 Hoyer, J., Schatzschneider, U., Schulz-Siegmund, M. \& Neundorf, I. Dimerization of a cell-penetrating peptide leads to enhanced cellular uptake and drug delivery. Beilstein J Org Chem 8, 1788-1797, doi:10.3762/bjoc.8.204 (2012).

40 Nakase, I. et al. Interaction of arginine-rich peptides with membraneassociated proteoglycans is crucial for induction of actin organization 
and macropinocytosis. Biochemistry 46, 492-501, doi:10.1021/bi0612824 (2007).

41 Verdurmen, W. P. et al. Preferential uptake of L- versus D-amino acid cell-penetrating peptides in a cell type-dependent manner. Chem Biol 18, 1000-1010, doi:10.1016/j.chembiol.2011.06.006 (2011).

42 Goncalves, E., Kitas, E. \& Seelig, J. Structural and thermodynamic aspects of the interaction between heparan sulfate and analogues of melittin. Biochemistry 45, 3086-3094, doi:10.1021/bi052221t (2006).

43 ur Rehman, Z., Sjollema, K. A., Kuipers, J., Hoekstra, D. \& Zuhorn, I. S. Nonviral gene delivery vectors use syndecan-dependent transport mechanisms in filopodia to reach the cell surface. ACS Nano 6, 75217532, doi:10.1021/nn3028562 (2012).

\subsection{Supplementary data}

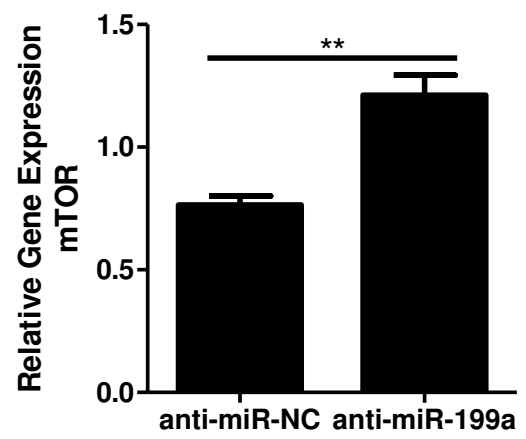

Figure S1. Nanocomplexes of anti-miR-199a oligonucleotides induce the target gene mTOR of miR-199a in human hPSCs. Gene expression of mTOR relative to housekeeping gene RPS18 in hPSCs. Statistical analysis was performed by two-tailed unpaired student $t$-test. Data represent mean \pm SEM., $n=3$. Statistical differences are $* * p<0.01$. 


\section{Chapter 4 - Reprogramming tumor stroma using an endogenous lipid lipoxin A4 to treat pancreatic cancer}

Jonas Schnittert $^{\mathrm{a}^{*}}$, Marcel A. Heinrich ${ }^{\mathrm{a}^{*}}$, Praneeth R. Kuninty ${ }^{\mathrm{a}}$, Gert Storm ${ }^{\mathrm{a}, \mathrm{b}}$, Jai Prakash $^{\mathrm{a}, \mathrm{c}, \S}$

a Section - Targeted Therapeutics, Department of Biomaterials Science and Technology, University of Twente, Enschede, The Netherlands. ${ }^{\mathrm{b}}$ Department of Pharmaceutics, Utrecht University, Utrecht, The Netherlands C ScarTec Therapeutics BV. Enschede, The Netherlands.

Published in Cancer Letters. 2018; 2018 April 28; volume 420

\section{Abstract}

Pancreatic stellate cells (PSCs) are the precursors of cancer-associated fibroblasts (CAFs), which potentiate pancreatic tumor growth and progression. In this study, we investigated whether Lipoxin A4 (LXA4), an endogenous bioactive lipid, can inhibit the differentiation of human PSCs (hPSCs) into CAFlike myofibroblasts and thereby hPSC-induced pro-tumorigenic effects. LXA4 significantly inhibited TGF- $\beta$-mediated differentiation of hPSCs by inhibiting pSmad2/3 signalling. Furthermore, treatment with LXA4 abolished the paracrine effects (proliferation and migration of Panc-1 tumor cells) of hPSCs in vitro. These data demonstrated that LXA4 can interrupt pro-tumoral paracrine signalling of hPSCs. Furthermore, LXA4 treatment significant decreased the size and growth rate of 3D-heterospheroids comprised of hPSC and Panc-1 and these effects were exhibited due to inhibition of hPSC-induced collagen 1 expression. In vivo, we examined the therapeutic efficacy of LXA4 in a co-injection (Panc1 and hPSCs) subcutaneous tumor model. Intriguingly, LXA4 significantly abolished the tumor growth (either injected intratumor or intraperitoneally), attributed to a significant reduction in fibrosis, shown with collagen 1 expression. Altogether, this study proposes LXA4 as a potent inhibitor for hPSCs which can be applied to reprogram tumor stroma in order to treat pancreatic cancer. 


\subsection{Introduction}

Annually, around 90,000 people in Europe and 45,000 people in the U.S. get diagnosed with pancreatic ductal adenocarcinoma (PDAC), the most common form of pancreatic cancer [1]. Patients with PDAC have a 1 year survival rate of $20 \%$, whereas the 5 -year survival rate decreases to $7 \%{ }^{1}$. In most PDAC, a surgical resection is not feasible, making radiotherapy or cytotoxic chemotherapy the golden standard treatment. However, these treatments are not effective and result in a survival benefit of only a few months ${ }^{2,3}$. One reason of the treatment failure is the abundant desmoplastic reaction in PDAC, also called the tumor stroma, which supports cancer progression, invasion and metastasis ${ }^{4}$. Novel therapeutic approaches for the treatment of PDAC aim to modulate the cellular components of the tumor stroma. Several different studies have shown an anti-tumoral effect by modulating the tumor stroma ${ }^{5,6}$, whereas unexpectedly a complete depletion of the stroma genetically was shown to promote tumor growth ${ }^{7}$. Therefore, it has been suggested to dampen the activity of tumor stroma instead of its total depletion to gain therapeutic benefits ${ }^{8}$.

Human pancreatic stellate cells (hPSCs) are the main precursors of pancreatic cancer-associated fibroblasts (CAFs), the key drivers of the pancreatic tumor stroma ${ }^{5,9,10}$. Quiescent hPSCs can be found within the connective tissue of the pancreas in low numbers and secrete only small amounts of extracellular matrix (ECM) proteins (e.g. collagen) ${ }^{11}$. In PDAC, quiescent hPSCs become activated, lose their cytoplasmic lipid storing capacity and start secreting high amounts of ECM components, leading to fibrosis which ultimately presents as a barrier for tumor drug penetration. In addition, differentiated hPSCs secrete various cytokines and growth factors which stimulate tumor cells and other stromal cells in favour of tumor progression ${ }^{12}$. Therefore, inhibition of hPSC activation might be beneficial for the treatment of pancreatic cancer.

Lipoxins are part of a family of inflammation resolving endogenous lipid mediators which are locally secreted by immune cells such as neutrophils and macrophages in response to infection, injury or inflammatory stimuli ${ }^{13-16}$. Lipoxins are synthesized from membrane arachidonic acid via biochemical synthesis by two major routes. The first route of synthesis starts in platelets where leukotriene A4 is converted into lipoxin by $12-\mathrm{LO}$. The second major route involves the action of either 5-LO in neutrophils and 15-LO in erythrocytes and reticulocytes. Arachidonic acid is converted into 15hydroperoxyeicosatetraenoic acid (15-HPETE), which is then converted into lipoxin $\mathrm{A}$ and lipoxin $\mathrm{B}{ }^{17}$. Based on an aspirin-triggered reaction in which 
aspirin acetylates cyclooxygenase-2 (COX-2) to form 15R-HPETE, and is eventually metabolized by 5-LO epi-lipoxin, lipoxin A4 (LXA4) and lipoxin B4 (LXB4) are formed ${ }^{18,19}$. Lipoxins and epi-lipoxins function by binding to the high-affinity G protein-coupled lipoxin A4 (LXA4) receptor formyl peptide receptor 2 (FPR2)/ ALX ${ }^{14}$. Via this route, LXA4 can resolve inflammation already at low nanomolar concentrations and additionally activates anti-bacterial mechanisms ${ }^{20,21}$.

There are little evidences that LXA4 may possess anti-fibrotic effects. In mesangial cells in kidney, it inhibited platelet-derived growth factor (PDGF)dependent TGF- $\beta$ production, the expression of fibrosis promoting genes in renal mesangial cells ${ }^{22}$ and Akt/PKB activation and cell cycle progression in mesangial cells ${ }^{23}$. Additionally, LXA4 attenuated experimental renal fibrosis ${ }^{24}$ and inhibited epithelial to mesenchymal transition of renal epithelial cells in proximal tubules ${ }^{25}$. Also, LXA4 has been shown to inhibit connective tissue growth factor-induced proliferation and TGF- $\beta$-dependent pro-fibrotic activity in human lung (myo)fibroblast ${ }^{13,26}$. Yet, there is no study showing the inhibitory effects of LXA4 on hPSC differentiation and its impact on pancreatic tumor growth.

Considering the anti-fibrotic activity of LXA4, in this study, we hypothesized that LXA4 might be an interesting biomolecule to inhibit PSCs activation and their pro-tumorigenic effects. We show that the LXA4 specific receptor FPR2/ALX is overexpressed in activated hPSCs. Furthermore, we demonstrate the inhibitory effect of LXA4 on the PSC activation, migration and then PSCinduced paracrine effect on pancreatic cancer cells. Next, we showed the inhibitory effects of LXA4 on the growth and extracellular matrix (ECM) deposition in stromal-rich 3D-heterospheroids. Eventually, we examined the therapeutic efficacy of LXA4 in vivo in a co-injection tumor model which resulted in a reduced tumor growth and intra-tumoral ECM deposition after the treatment with LXA4.

\subsection{Materials and methods:}

\subsubsection{Cells}

Primary human pancreatic stellate cells (ScienCell, Carlsbad, USA), were cultured in complete Stellate Cell Medium (supplemented with $2 \%$ FBS, $1 \%$ Penicillin/Streptomycin and $1 \%$ Stellate Cell Growth Supplements (SteCGS)) (ScienCell). Panc-1 cancer cells were cultured in Dulbecco's Modified Eagles 
medium (DMEM) High Glucose (4.5 g/l) with L-Glutamine (GE Healthcare, Vienna, Austria) supplemented with $10 \%$ FBS (Lonza, Verviers, Belgium), 100 $\mu \mathrm{g} / \mathrm{ml}$ penicillin/streptomycin (Sigma Aldrich). AsPc-1 cells were cultured in complete RPMI Medium (GE Healthcare, Vienna, Austria) supplemented with $10 \%$ FBS (Lonza), $100 \mathrm{ug} / \mathrm{ml}$ penicillin/streptomycin (Sigma). The cells were maintained at $37{ }^{\circ} \mathrm{C}$ in a humidified $5 \% \mathrm{CO} 2$ atmosphere

\subsubsection{Western blot}

To evaluate the expression of the LXA4-specific surface receptor FPR2 and the hPSC activation marker $\alpha$-SMA, hPSCs were seeded into a 12 well plate at a seeding density of 40.000 cells/well. The next day hPSCs were starved for $24 \mathrm{~h}$ and subsequently activated with $5 \mathrm{ng} / \mathrm{ml} \mathrm{TGF}-\beta$ for $48 \mathrm{~h}$. To evaluate the effect of LXA4 on TGF- $\beta$-mediated Smad $2 / 3$ and phosphorylated Smad 2/3 (pSmad2/3) levels in hPSCs, cells were seeded into a 12 well plate at a seeding density of 40.000 cells/well. The cells were starved for $24 \mathrm{~h}$, treated with $10 \mathrm{nM}$ LXA4 (Biomol, Hamburg, Germany) and activated with $5 \mathrm{ng} / \mu \mathrm{l}$ TGF- $\beta$ for 30 min. In general, cells were lysed using $1 \times$ SDS-lysis buffer, cell lysis was centrifuged at 10,000 $\mathrm{g}$ for $10 \mathrm{~min}$, and the supernatant was collected for Western blot analysis. To analyse the expression of MMP-2 in conditioned medium, $1 \mathrm{ml}$ conditioned medium was freeze dried using an Il-Shin TFD5503 Freeze Dryer (Scala Scientific, Ede, The Netherlands) and afterwards resuspended in $100 \mu \mathrm{l}$ TBS (Thermo Scientific, Rockford, USA). Conditioned medium was mixed with Sample Reducing agent (Life Technologies, Carlsbad, USA) and LDS Sample Buffer (Life Technologies) and incubated at $95{ }^{\circ} \mathrm{C}$ for $10 \mathrm{~min}$. Protein lysates and conditioned medium was loaded on a $10 \%$ Tris-Glycine gel (Thermo Scientific) and transferred onto PVDF membranes (Thermo Scientific). The blots were incubated with the required primary antibody (Supplementary Table 1) and incubated overnight at $4{ }^{\circ} \mathrm{C}$, followed by incubation with species specific horseradish peroxidase (HRP) conjugated secondary and tertiary antibody for $1 \mathrm{~h}$ at RT. Proteins were detected with Pierce ${ }^{\mathrm{TM}}$ ECL Plus Western Blotting Substrate kit (Thermo Scientific) and exposed to FluorChem ${ }^{\mathrm{TM}}$ M System (ProteinSimple, San José, USA). The protein levels were quantified using Image J software (NIH, MD). To evaluate the amount of Smad 2/3 phosphorylation a pSmad 2/3/Smad 2/3 ratio was calculated.

\subsubsection{Immunocytochemistry}

To evaluate the effect of LXA4 on the expression of $\alpha \mathrm{SMA}$ and collagen 1 (Col1), hPSCs were seeded into a 24 well plate at a seeding density of 5.000 cells/well. The cells were starved, treated with $0.1,1$ and $10 \mathrm{nM} \mathrm{LXA} 4$ and 
TGF- $\beta$ activated for $48 \mathrm{~h}$. Next, cells were fixed and immunostained for $\alpha$ SMA, and collagen 1 as described elsewhere [27]. Stained images were quantified for their positively stained area using ImageJ software.

To evaluate the effect of LXA4 on Smad2/3 and pSmad2/3 expression, hPSCs were seeded into a 24 well plate at a seeding density of 10.000 cells/well. The cells were starved and activated with TGF- $\beta$ as described previously. After 30 min of incubation with TGF- $\beta$, cells were washed with phosphate buffered saline (PBS) (Lonza, Verviers, Belgium) and fixed with 4\% paraformaldehyde (Sigma Aldrich) at room temperature for $10 \mathrm{~min}$. Next, cells were washed 3 times with PBS and permeabilized with ice cold HPLC grade methanol (Fisher Scientific) at $-20{ }^{\circ} \mathrm{C}$ for $10 \mathrm{~min}$. After washing 3 times with PBS cells were blocked with blocking buffer consisting of 1 vol \% bovine-serum albumin (BSA) (VWR, Radnor, PA, USA) +0.3 vol \% Triton-X (Sigma Aldrich) in PBS for $1 \mathrm{~h}$. Subsequently, the cells were incubated with monoclonal anti-Smad $2 / 3$ or monoclonal anti-pSmad2/3 primary antibodies diluted in blocking buffer and incubated over night at $4{ }^{\circ} \mathrm{C}$. Next, cells were washed 3 times with PBS and incubated with fluorescent secondary antibodies diluted in blocking buffer for 1 $h$ at RT. Cells were washed once with PBS and stained for DAPI by incubation with NucBlue Fixed Cell Stain ReadyProbes Reagent (Life Technologies), diluted in PBS as described in the manufacturer's instructions, for $5 \mathrm{~min}$. The NuCBlue solution was replaced with PBS and the cells imaged for Alexa Fluor 488 (ex. 495/em. 520) and DAPI (ex. 360/em. 460) using an EVOS FI fluorescent microscope (Life Technologies). Staining of $\mathrm{pSmad} 2 / 3$ was quantified by counting the total number and the number of $\mathrm{pSmad} 2 / 3$ positive nuclei.

\subsubsection{F-Actin staining}

To analyse the effect of LXA4 on the F-actin organization of TGF- $\beta$ activated hPSCs, hPSCs were seeded into 24 well plates at a seeding density of 2.500 cells/well. After $24 \mathrm{~h}$ of incubation with $10 \mathrm{nM} \mathrm{LXA} 4$ and / or $5 \mathrm{ng} / \mathrm{ml}$ TGF- $\beta$, cells were washed three times with PBS and subsequently fixed in PBS containing $10 \%$ formaldehyde (Sigma Aldrich) for $15 \mathrm{~min}$. After permeabilizing the cells with $0.1 \mathrm{M}$ Triton X-100 (Sigma Aldrich) for $5 \mathrm{~min}, \mathrm{~F}$-actin was stained with phalloidin (Life Technologies) at a concentration of $250 \mathrm{ng} / \mathrm{ml}$ for $30 \mathrm{~min}$. Next, cells were washed with PBS and imaged for phalloidin at (ex. 540/em. 565) using an EVOS FI fluorescent microscope (Life Technologies). 


\subsubsection{Quantitative real time PCR}

To evaluate the effect of LXA4 on TGF- $\beta$-mediated hPSCs activation, cells were seeded into a 12 well plate at a seeding density of 40.000 cells/well. The cells were starved for $24 \mathrm{~h}$, treated with $10 \mathrm{nM} \mathrm{LXA} 4$ and activated with $5 \mathrm{ng} / \mu \mathrm{l} \mathrm{TGF}$ B. $24 \mathrm{~h}$ after activation total RNA was isolated using the GenElute ${ }^{\mathrm{TM}}$ Mammalian Total RNA Miniprep Kit (Sigma Aldrich) and the RNA amount was measured by a NanoDrop ${ }^{\circledR}$ ND-1000 Spectrophotometer (Thermo Scientific). Subsequently, cDNA was synthesized with iScript ${ }^{\mathrm{TM}}$ cDNA Synthesis Kit (BioRad, Veenendaal, The Netherlands). $10 \mathrm{ng}$ cDNA were used for each PCR reaction. The real-time PCR primers (Table 1) were purchased from (Sigma Aldrich). Quantitative real time PCR was performed with the 2x SensiMix SYBR and Fluorescein Kit (Bioline GmbH, Luckenwalde, Germany) using a BioRad CFX384 Real-Time PCR detection system (BioRad). Gene expression levels were normalized to the expression of the house-keeping gene RPS18.

Table 1. Sequences of forward and reverse primers used during real-time PCR.

\begin{tabular}{|l|l|l|}
\hline Gene & Forward Primer & Reverse Primer \\
\hline RPS18 & TGAGGTGGAACGTGTGATCA & CCTCTATGGGCCCGAATCTT \\
\hline Acta- 2 & CCCCATCTATGAGGGCTATG & CAGTGGCCATCTCATTTTCA \\
\hline Collagen $1 \alpha 1$ & GTACTGGATTGACCCCAACC & CGCCATACTCGAACTGGAAT \\
\hline Collagen $3 \alpha 1$ & AAGAAGGCCCTGAAGCTGAT & GTGTTTCGTGCAACCATCCT \\
\hline PDGF $\beta R$ & AGGCAAGCTGGTCAAGATCT & GCTGTTGAAGATGCTCTCCG \\
\hline MMP2 & AGGAGGAGAAGGCTGTGTTC & CTCCAGTTAAAGGCGGCATC \\
\hline WNT2 $\beta$ & TTTAGGTGCTGGTGGGAGAG & CTCTTCTTCACCTGGGAGCA \\
\hline IDI 1 & TTGGGCTGGATAAAACCCCT & GCAACATCCGGCATAACTGT \\
\hline NSDHL & ATGCTATGGAGAGGACCGTG & CAGAAGGAGTCACAGGCTCA \\
\hline INSIG1 & GGCAGCTTCCCAAGTATTCG & CTACCTCCTTTGGGCACTGA \\
\hline CXCL12 & TGCCCTTCAGATTGTAGCCC & GCGTCTGACCCTCTCACATC \\
\hline CTGF & GTTTGGCCCAGACCCAACTA & GGCTCTGCTTCTCTAGCCTG \\
\hline
\end{tabular}

\subsubsection{Conditioned media}

To collect hPSC conditioned medium, cells were seeded into a 12 well plate at a seeding density of $40.000 \mathrm{cells} /$ well. As previously described, cells were starved, treated with LXA4 and activated with TGF- $\beta$ for $24 \mathrm{~h}$. Next, the cells were washed 3 times with serum-free medium. After $48 \mathrm{~h}$ of incubation with serumfree medium the medium was collected for use in conditioned medium experiments. 


\subsubsection{Cell proliferation assay}

hPSCs were seeded into a 96 well plate at a density of 2.500 cells/well. The next day, hPSCs were treated with $10 \mathrm{nM}$ of LXA4. Cell number was monitored every $24 \mathrm{~h}$ over a time period of $72 \mathrm{~h}$. To monitor the cell number, $10 \mu \mathrm{l}$ of Alamar Blue dye (Invitrogen, Carlsbad, USA) in $100 \mu \mathrm{l}$ of hPSC growth media was added per well. After $4 \mathrm{~h}$ the fluorescent signal was measured using a VIKTOR $^{\text {TM }}$ plate reader (Perkin Elmer, Waltham, Massachusetts). To evaluate the effect of hPSC conditioned media on Panc-1 and AsPc-1 cell growth, Panc1 and AsPc-1 were seeded in a 96 well plate at a density of 5000 cells $/$ well. $24 \mathrm{~h}$ after seeding the previously collected condition media was applied to the cells. Cell number was monitored at $0 \mathrm{~h}, 24 \mathrm{~h}$ and $48 \mathrm{~h}$ as described previously.

\subsubsection{Wound healing assay}

To evaluate the effect of LXA4 on hPSC migration, cells were seeded into a 24 well plate at a seeding density of 50.000 cells/well. As previously described cells were starved, treated with LXA4 and activated with TGF- $\beta$ for $24 \mathrm{~h}$. A scratch was made on the culture plate using a $200 \mu \mathrm{l}$ pipette tip fixed in a custom-made holder. Next, cells were washed and incubated with fresh serum-free media. Images were captured at $\mathrm{t}=0 \mathrm{~h}$ and $\mathrm{t}=12 \mathrm{~h}$, with an EVOS microscope. Images were analysed by Image $\mathbf{J}$ software to calculate the area of the scratch and represented as percentage of wound closure.

\subsubsection{Transwell migration Assay}

To evaluate the effect of hPSC conditioned media on Panc-1 and AsPc-1 migration, Panc-1 and AsPc-1 were seeded into the upper chamber of a 24 well Transwell insert (Sigma Aldrich) at a seeding density of 50.000 cells/well. After $24 \mathrm{~h}$, the cells were fixed with ice cold methanol (Fisher Scientific) for $10 \mathrm{~min}$. Next, the cells were incubated with $0.1 \%$ Crystal Violet solution (Sigma Aldrich) in 25\% methanol (Fisher Scientific) for $10 \mathrm{~min}$. After washing the cells with MilliQ water, cells from the upper compartment were removed with a cotton swap and the inserts were allowed to dry. The migrated cells were imaged with a Nikon microscope (Nikon Eclipse E400, Tokyo, Japan). Images were analysed by Image $\mathbf{J}$ software to calculate the positively stained area.

\subsubsection{Spheroid formation assay}

3D-heterospheroids containing a mixture of hPSCs and Panc-1 cells were prepared using the hanging drop method. hPSCs and Panc-1 were trypsinized and suspended in their respective culture medium to a concentration of 300.000 cells $/ \mathrm{ml}$. The hPSCs and Panc-1 cell suspensions were mixed 1:1 (v/v). 
For spheroid formation, a drop of $20 \mu$ containing 6000 cells were dispensed onto a lid of a round bottom suspension culture 96-well plate (Greiner BioOne, Alphen aan den Rijn, The Netherlands). Next, the lid was inverted and placed onto the 96-well plate. Wells were filled with $150 \mu \mathrm{l}$ PBS for humidity. For spheroids prepared with LXA4-treated hPSCs, hPSCs were seeded, starved for $24 \mathrm{~h}$ and treated with $10 \mathrm{nM}$ LXA4 for an additional $24 \mathrm{~h}$. The spheroids were grown for eight days. Spheroids which were LXA4-treated after formation were allowed to form for 4 days. Next, $10 \mu \mathrm{l}$ of $20 \mathrm{nM}$ LXA4 was directly added into the droplet. The spheroids were grown for an additional 8 days. Spheroids were imaged under an inverted microscope, and their area was measured digitally using ImageJ software.

\subsubsection{Immunohistochemical staining of 3D-heterosperoids}

For sectioning 3D-heterospheroids were collected, embedded in Cryomatrix (Thermo Scientific) and frozen at $-80^{\circ} \mathrm{C}$. Afterwards, spheroids were cut into $6 \mu \mathrm{m}$ thick sections using a Cryotome FSE (Thermo Scientific). The spheroids collected on microscope slides were rehydrated in PBS for 5 min before fixation in $4 \%$ paraformaldehyde (Sigma Aldrich) for $30 \mathrm{~min}$. Next, the slides were washed 3 times with PBS ( 5 min each). The edges of the slides were marked with a hydrophobic Pap-pen (Life Technologies). After washing, the cells were permeabilized with 0.1 vol \% Triton-X (Sigma Aldrich) in PBS for 15 min and washed again. Slides were incubated with the primary antibody (Supplementary Table 2) diluted in PBS overnight at $4{ }^{\circ} \mathrm{C}$. The next day, the slides were washed 3 times with PBS before the secondary fluorescent antibody (see Supplementary Table 2) diluted in PBS supplemented with 5 vol\% of normal human serum (Thermofisher Scientific) were added. Finally, the slides were washed and mounted with fluoroshieldTM with DAPI (Sigma Aldrich) for staining of nuclei. Sections have been imaged using a Hamamatsu NanoZoomer Digital slide scanner 2.0HT (Hamamatsu Photonics, Bridgewater NJ).

\subsubsection{Animal study}

Six-week-old male CB17 SCID mice (Janvier Labs, Le Genest-Saint-Isle, France) were subcutaneously injected with a mixture of $2 \times 106$ Panc-1 and $4 \times 106$ of hPSCs. Tumor formations and volumes were assessed every $3-4$ days. Tumor volumes were calculated using a standard formula [volume $=1 / 2$ (length $\mathrm{x}$ width $\left.{ }^{2}\right)$ ]. The treatment was started 9 days after injection of the tumors. Six tumor-bearing mice per treatment groups were taken and injected with either vehicle or LXA4 twice a week. Of each group half were injected intraperitoneal 
$(40 \mu \mathrm{g} / \mathrm{kg})$ and another half local intratumoral $(4 \mu \mathrm{g} / \mathrm{kg})$. At the end of the experiments, animals were sacrificed under anesthesia, tumors were harvested and immediately snap frozen in cold 2-methyl butane (Fisher Scientific). Frozen tumors were stored at $-80^{\circ} \mathrm{C}$ until analysis.

\subsubsection{Immunohistochemical staining of mouse tumors}

Mouse tumors were embedded in Cryomatrix (Thermo Scientific) and frozen at - $80^{\circ} \mathrm{C}$. Afterwards, spheroids were cut into $6 \mu \mathrm{m}$ thick sections using a Cryotome FSE (Thermo Scientific). The tumor section were dried and fixed in acetone (Fisher Scientific) for $10 \mathrm{~min}$. The edges of the slides were marked with a hydrophobic Pap-pen (Life Technologies). Next, the tumor sections were rehydrated in PBS for $10 \mathrm{~min}$. Tumor sections were incubated with the primary antibody (Supplementary Table 2) diluted in PBS overnight at $4{ }^{\circ} \mathrm{C}$. The next day, the slides were washed 3 times with PBS before the secondary fluorescent antibody or HRP-labeled antibody (Supplementary Table 2) diluted in PBS supplemented with 5 vol\% of normal mouse serum (Thermofisher Scientific) were added (normal human serum was used for $\alpha$-SMA staining). Staining with HRP-labeled secondary antibodies was continued as described elsewhere ${ }^{27}$. For staining with fluorescent antibodies, the slides were washed and mounted with fluoroshieldTM with DAPI (Sigma Aldrich) for staining of nuclei. Sections have been imaged using a Hamamatsu NanoZoomer Digital slide scanner 2.0HT (Hamamatsu Photonics, Bridgewater NJ).

\subsubsection{Immunohistochemical staining of human tumors}

Human paraffin embedded PDAC sections were deparaffinized in xylene, rehydrated in serially diluted alcohol solutions, followed by demineralized water. Antigen retrieval was performed by at $80{ }^{\circ} \mathrm{C}$ using Tris buffer $(\mathrm{pH} 9.0$, Dako, Glastrup, Denmark) for $16 \mathrm{~h}$. To block endogenous peroxidase, slides were incubated in $0.3 \%$ hydrogen peroxide in phosphate-buffered saline (PBS) for $20 \mathrm{~min}$. Next, slides were incubated for $2 \mathrm{~h}$ with an antibody against $\alpha$-SMA (A2547 Sigma, the Netherlands), followed by incubation with the secondary antibody for $60 \mathrm{~min}$ at room temperature. The immunohistochemical staining was visualised using 3.3-diaminobenzidine tetrahydrochloride solution (Dako) for 5-10 min resulting in a brown staining and then counterstained with hematoxylin, dehydrated and finally mounted in vectamount.

\subsubsection{Graphs \& statistical analysis}

All graphs were made using GraphPad Prism Vol.5 (GraphPad Software Inc., San Diego, CA). All values are expressed as a mean \pm standard error of the mean 
(SEM). Statistical significance of the results was performed by either a twotailed unpaired student's t-test for comparison of two treatment groups or a oneway ANOVA to compare multiple treatment groups. Differences were considered significant for a $\mathrm{p}$-value of $* \mathrm{p}<0.05$, **p $<0.01$, ***p $<0.001$, respectively.

\subsection{Results}

\subsubsection{LXA4 expression is induced in TGF- $\beta$ activated hPSCs}

A

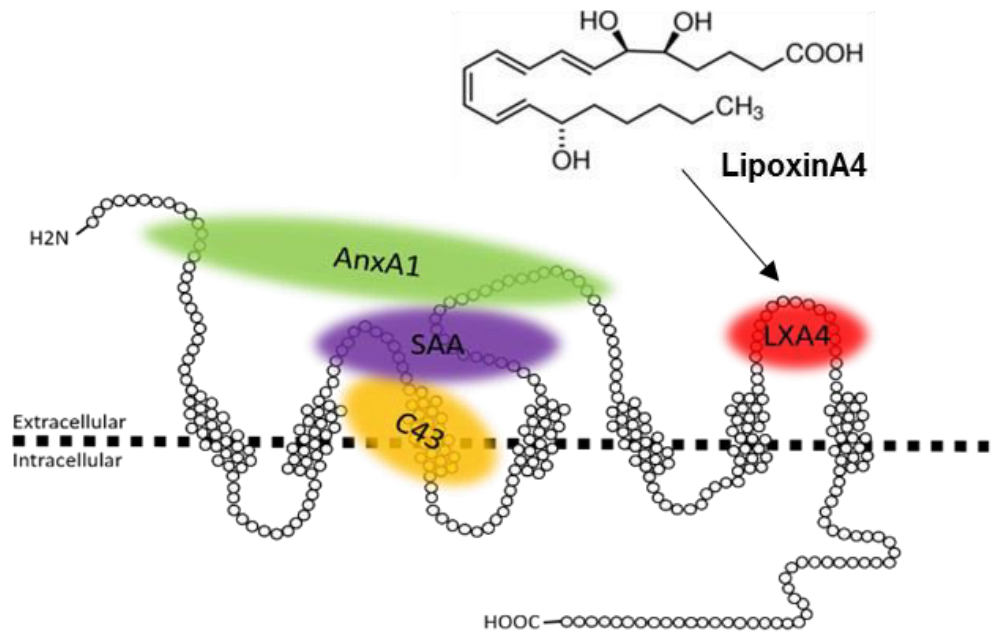

B

C

D
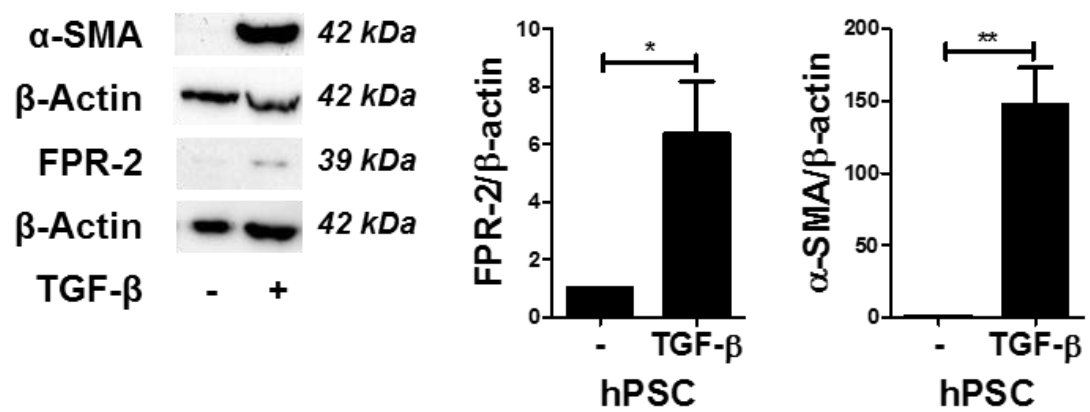

Fig. 1. Structure and protein expression of the Lipoxin A4 (LXA4) specific surface receptor FPR2 on hPSCs. Structure of the LXA4-specific surface receptor FPR2 displaying the binding site of LXA4 (A). Protein expression of the LXA4-specific surface receptor FPR2 $(B, C)$ and the activation marker $\alpha-S M A(B, D)$ in hPSCs and hPSCs activated with TGF- $\beta$ for 48 h (B). Data represents mean \pm SEM for at least 3 independent experiments. Statistical analysis was performed by two-tailed unpaired student $t$-test. Statistical differences are $* p<0.05, * *<0.01$. 
LXA4 binds to the extracellular domain of the N-formyl-peptide receptor 2 (FPR2) receptor as shown in Fig. 1 A. To achieve the effect of LXA4 on hPSCs, it is crucial to confirm whether these cells express the FPR2 receptor in their quiescent and active state. Therefore, we activated hPSCs with TGF- $\beta$ for $48 \mathrm{~h}$ and examined the protein expression of FPR2 using western blot analysis. As shown in Fig. 1 B, the expression of FPR2 receptor was induced by about 6.5fold in the TGF- $\beta$-activated hPSCs compared to the non-activated form (Fig. 1 B, C). To confirm the activation of hPSCs with TGF- $\beta$ we have performed a western blot for $\alpha$-SMA, showing the significant induction of this CAF marker upon TGF- $\beta$ treatment (Fig. 1 B, D).

\subsubsection{LXA4 inhibits TGF- $\beta$ induced PSC differentiation}

As shown in Fig. $2 \mathrm{~A}$ and D, after activation with TGF- $\beta$, hPSCs attained a myofibroblast phenotype, as can be seen with their high expression of $\alpha$-SMA and collagen1 (Col1) and stretched morphology.
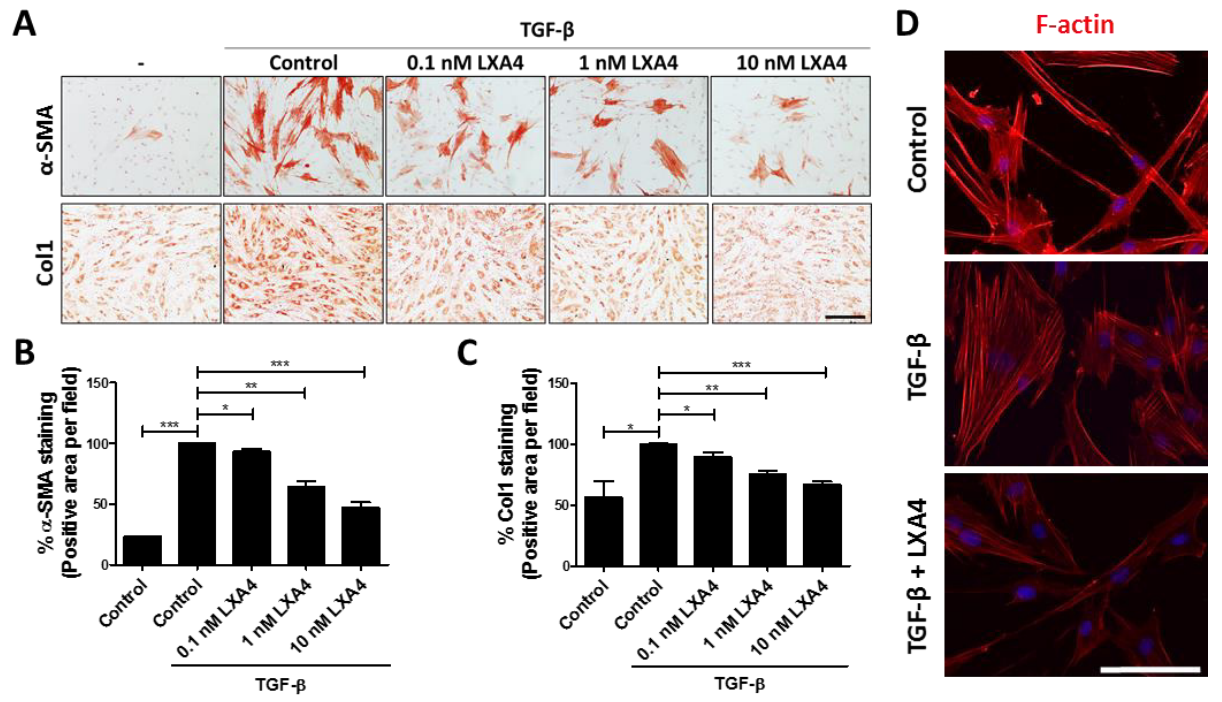

Fig. 2. Lipoxin A4 effect on TGF- $\beta$ mediated hPSC differentiation. Immunostaining showing the inhibitory effect of $L X A 4(0.1,1,10 \mathrm{nM})$ on the protein expression of $\alpha-S M A$ and Coll (A). Scale bar $=200 \mu \mathrm{m}$. Quantification of $\alpha-S M A(B)$ and Coll $(C)$ staining for the respective concentrations of LXA4. F-actin staining showing morphological changes in TGF- $\beta$ activated and LXA4-treated and TGF- $\beta$ activated compared to normal hPSCs (D). Control contains $0.001 \%$ ethanol. Data represent mean + - SEM for at least 3 independent experiments. Statistical analysis was performed by two-tailed unpaired student $t$-test. $* p<0.05, * * p<0.01, * * * p<0.001$. 
Treatment with LXA4 not only significantly inhibited the protein expression of $\alpha$-SMA and Col1 in a concentration dependent manner, but also inhibited the change in the morphology (Fig. $2 \mathrm{~A}, \mathrm{D}$ ). The quantitation of stainings confirmed the reduction in the expression of both $\alpha$-SMA and Coll with the most profound effects at a concentration of $10 \mathrm{nM}$ (Fig. $2 \mathrm{~B}, \mathrm{C})$.

A

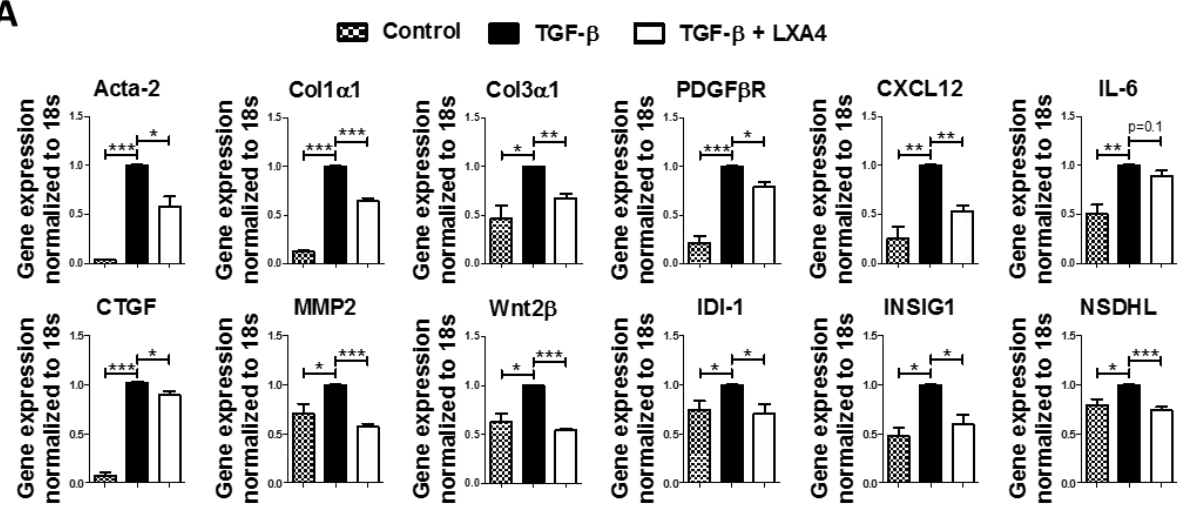

B

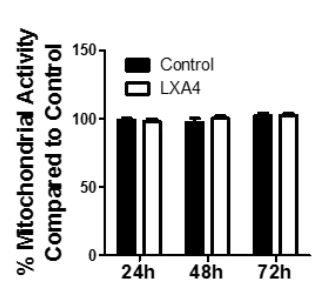

C

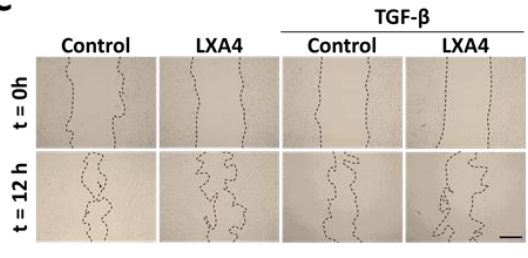

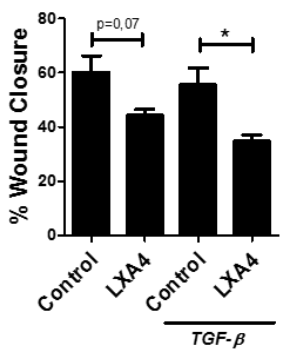

Fig. 3. Lipoxin A4 effect on TGF-ק mediated hPSC differentiation, viability and migration potential. Gene expression of hPSC activation markers encoding growth factors (CTGF), membrane receptors (PDGFBR), chemokines (IL-6 and CXCL12), enzymes (MMP2), proteins involved in cholesterol production and homeostasis (IDI1, INSIGI and NSDHL), intracellular proteins ( $\alpha-S M A, W n t 2 \beta)$ and ECM proteins (Coll $\alpha 1$, Col3 $\alpha 1)$ displaying the inhibitory effect of $10 \mathrm{nM}$ LXA4 after treatment with $5 \mathrm{ng} / \mathrm{ml}$ TGF- $\beta$ LXA4 (A). Mitochondrial activity of hPSCs treated with $10 \mathrm{nM}$ Lipoxin $A 4$ and EtOH control after 24, 48 and $72 \mathrm{~h}$ of incubation (B). Representative microscopic images and quantification showing the effect of LXA4 $(10 \mathrm{nM})$ on the migration of $h P S C s$ and hPSCs activated with TGF- $\beta$ (C). Scale bar $=500 \mu \mathrm{m}$. Control contains $0.001 \%$ ethanol. Data represent mean + - SEM for at least 3 independent experiments. Statistical analysis was performed by two-tailed unpaired student t-test. ${ }^{*} p<0.05,{ }^{* *} p<0.01$, $* * * p<0.001$. 
Interestingly, LXA4 at $10 \mathrm{nM}$ concentration significantly inhibited the TGF- $\beta$ induced expression of genes encoding for the CAF phenotype, including growth factors (CTGF and CXCL12), membrane receptors (PDGF $\beta R$ ), cytokines inducing cancer cell growth (IL-6), enzymes facilitating increased ECM turnover and altered ECM deposition (MMP2), genes involved in cholesterol production and homeostasis (IDI1, INSIG1, NSDHL), intracellular proteins (Acta-2 (encoding for $\alpha$-SMA), Wnt2B) and ECM proteins (Col1 $\alpha 1$, Col3 $\alpha 1$ ) (Fig. 3 A). Additionally, we detected a significant reduction in TGF- $\beta$-induced expression of secreted MMP-2 in the conditioned medium of hPSCs treated with $10 \mathrm{nM}$ of LXA4 (Supplementary Fig. 2). Of note, treatment of hPSCs with $10 \mathrm{nM}$ LXA4 over a period of $72 \mathrm{~h}$ exerted no toxic effects on their metabolic activity (Fig. 3 B). Next, we examined the effect of LXA4 on the migration of hPSCs using a scratch (wound healing) assay. The migration potential in TGF- $\beta$-activated hPSCs was not induced compared to untreated hPSCs (Fig. 3 C). LXA4 led to a significant inhibition of the wound closure in both non-activated and TGF- $\beta$ activated hPSCs compared to control cells (Fig. 3 C). These data indicate that LXA4 inhibits the activation of hPSCs with TGF- $\beta$ to attain the CAF-like myofibroblast phenotype.

\subsubsection{LXA4 inhibits TGF- $\beta$ induced smad $2 / 3$ nuclear translocation and phosphorylation}

Since LXA4 inhibited the TGF- $\beta$-mediated activation of hPSCs, we examined the effect of LXA4 on the TGF- $\beta$ signaling pathway. After binding to its receptor, TGF- $\beta$ activates the Smad $2 / 3$ pathway, whereby Smad $2 / 3$ gets phosphorylated and locates within the nucleus. As shown in Fig. 4 A using immunofluorescent microscopy, in non-activated hPSCs, Smad2/3 was localized in the cytoplasm but not in the nuclei, while after 30 min incubation with TGF$\beta$, Smad 2/3 translocated into the nuclei (Fig. 4 A). Treatment with LXA4 reduced the translocation into the nuclei (Fig. 4 A). To confirm the activation state of Smad2/3 and quantify this, we examined the phosphorylated Smad2/3 (pSmad2/3) using immunofluorescent staining which clearly showed a significant translocation of $\mathrm{pSmad} 2 / 3$ into the nuclei of hPSCs upon TGF- $\beta$ activation compared to untreated cells (Fig. 4 A). Importantly, the nuclear translocation of $\mathrm{pSmad} 2 / 3$ was significantly inhibited by LXA4, as shown in the immunofluorescent pictures and quantitative data (Fig. 4 A, B). Additionally, we have performed a Western Blot to analyse the protein expression of Smad 2/3 and pSmad 2/3 (Supplementary Figure $1 \mathrm{~A}$ ) and found a significant reduction in the pSmad 2/3/Smad 2/3 ratio (Supplementary Figure $1 \mathrm{~B}$ ), confirming the 
inhibition of Smad 2/3 phosphorylation, which we previously had observed in immunofluorescent staining.

A

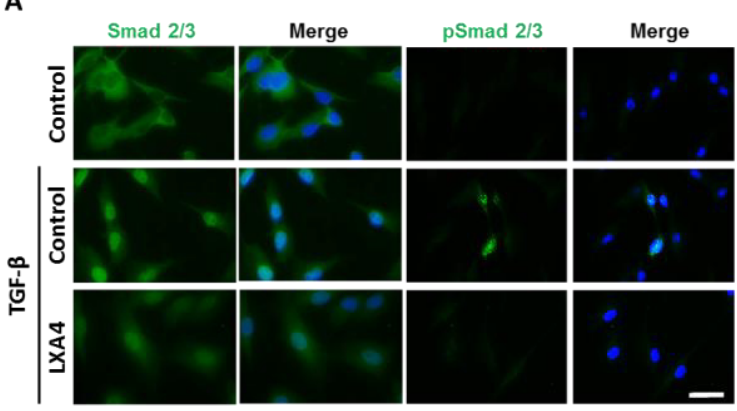

B

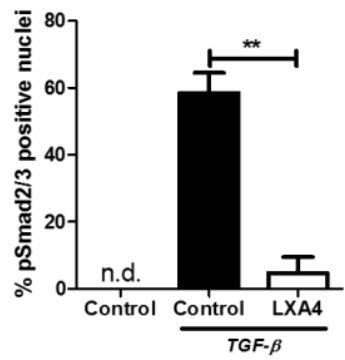

Fig. 4. Effect of LXA4 on Smad $2 / 3$ phosphorylation in TGF- $\beta$ activated hPSCs. Immunofluorescent staining of Smad 2/3 and pSmad 2/3 in hPSCs, TGF- $\beta$ activated hPSCs treated with control or $10 n M$ LXA4 (A). Scale bar $=50 \mu \mathrm{m}$. Quantification of fluorescent pSmad 2/3 staining showing the percentage of pSmad 2/3 positive nuclei $(B)$. Control contains $0.001 \%$ ethanol. Data represent mean + SEM for at least 3 independent experiments. Statistical analysis was performed by two-tailed unpaired student t-test. $* * p<0.01$.

\subsubsection{LXA4 inhibits hPSC-mediated paracrine effects on tumor cells}

After confirming the direct effect of LXA4 on hPSCs, we investigated the hPSCinduced paracrine effect on the growth and migration potential of human pancreatic tumor cells (Panc-1 \& AsPc-1). To study that, we collected conditioned media from hPSCs with or without activation with TGF- $\beta$ to collect hPSC-secreted cytokines and growth factors (Fig. 5 A). Incubation of Panc1 cells with the conditioned media from TGF- $\beta$-activated hPSCs showed an induced Panc-1 cell growth and migration compared to the conditioned media from non-activated hPSCs (Fig. 5 B, C). Interestingly, conditioned media collected from LXA4-treated hPSCs showed reduced Panc-1 cell growth and migration (Fig. 5 B, C). For AsPc-1 tumor cells we observed the same trend, with regard to their migration and cell growth, in LXA4-treated hPSCs (Supplementary Fig. 3 A, B) (Supplementary Figure 3 D). Next, we wanted to study the effect of LXA4 on hPSCs in direct contact of Panc-1 cells using a 3Dheterospheroid co-culture system, as shown earlier by us elsewhere ${ }^{28}$. 
A

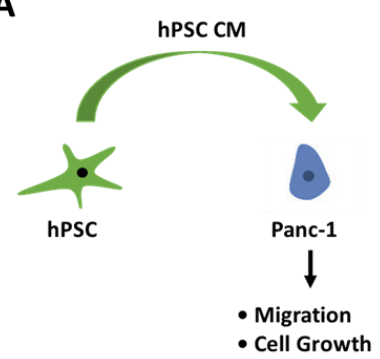

D

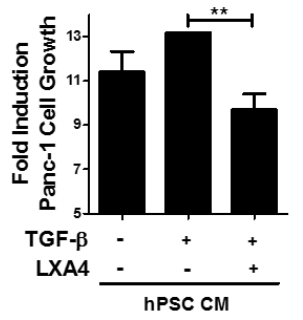

G

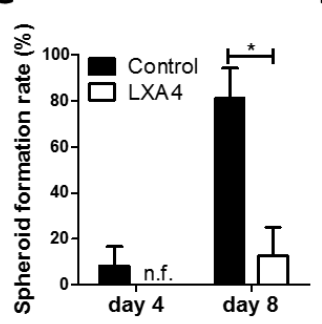

H
B

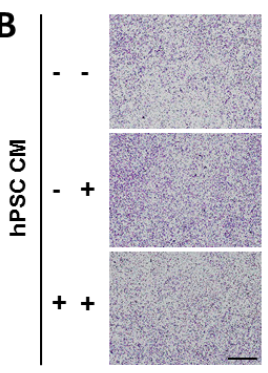

荄是是
C

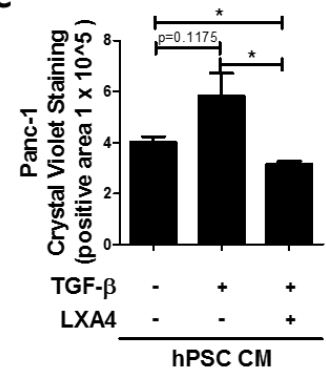

$\mathbf{F}$

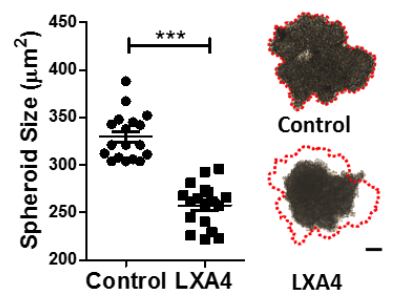

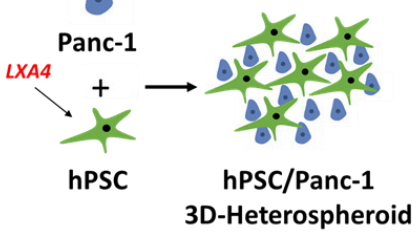
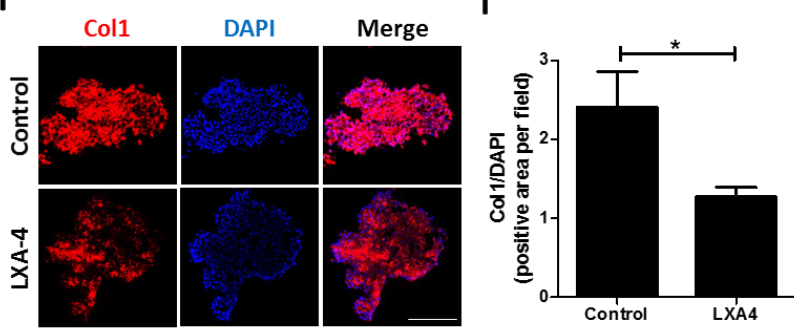

Fig. 5. Effect of LXA4 on hPSC-induced tumor cell migration, proliferation and 3Dheterospheroid formation. Schematic representation of the hPSC conditioned medium experiment (A). Representative images $(B)$ and quantification $(C)$ of Panc-1 tumor cell migration after $24 \mathrm{~h}$ of incubation with condition medium collected from untreated, control-treated TGF- $\beta$ activated hPSCs and $10 \mathrm{nM}$ LXA4-treated TGF- $\beta$ activated hPSCs. Scale bar $=400 \mu \mathrm{m}$. Panc-1 epithelial tumor cell growth after $48 \mathrm{~h}$ of incubation with condition medium collected from untreated, control-treated TGF- $\beta$ activated hPSCs and 10 nM LXA4-treated TGF- $\beta$ activated hPSCs (D). Schematic representation of spheroid formation (E). Size of hPSC/Panc-1 3D-heterospheroid formed with controltreated or LXA4-treated hPSCs after 8 days, including representative images of hPSC/Panc-1 3D-heterospheroids formed with control-treated of LXA4-treated hPSCs (F). Scale bar $=100 \mu \mathrm{m}$. Formation rate in percent of hPSC/Panc-1 3D-heterospheroid formed with control-treated of LXA4-treated hPSCs after 4 and 8 days $(G)$. Immunofluorescent staining on $6 \mu \mathrm{m}$ thick sections of hPSC/Panc-1 3D-heterospheroid formed with control-treated of LXA4-treated hPSCs for the expression Coll and nuclei staining (DAPI) (H). Scale bar $=200 \mu \mathrm{m}$. Quantification of Col1 staining divided by 
DAPI staining of hPSC/Panc-1 3D-heterospheroid formed with control-treated of LXA4treated hPSCs (I). Control contains $0.001 \%$ ethanol. Data represent mean + SEM for at least 3 independent experiments. Statistical analysis was performed by two-tailed unpaired student t-test. $* p<0.05, * * p<0.01$, ***p $<0.001$.

In order to investigate the effect of LXA4 on 3D-heterospheroid formation and growth, we formed spheroids with hPSC that were treated with LXA4 prior to the experiment (Fig. 5 E). Properly formed spheroids were imaged 8 days after formation using bright-field microscopy and measured for their size (Fig. 5 F). The results showed a significant reduction in spheroid size when hPSCs were treated with LXA4 before spheroid formation when compared to spheroids formed with EtOH-treated hPSCs (Fig. 5 E). Strikingly, spheroids formed with LXA4-treated hPSCs were not yet formed at day 4 after the initiation of spheroid formation, while spheroids containing EtOH-treated hPSCs had already formed (Fig. $5 \mathrm{G}$ ). After 8 days the rate of 3D-heterospheroid formation was $\sim 80 \%$ for spheroids formed with EtOH-treated hPSCs and $\sim 15 \%$ for spheroids formed with LXA4-treated hPSCs (Fig. 5 G). To investigate whether LXA4 does also inhibit hPSC activation in 3D-heteropheroids, we performed immunofluorescent staining on $6 \mu \mathrm{m}$ thick sections of hPSC/Panc-1 3D-heterospheroids (Fig. $5 \mathrm{H}$ ). We found that spheroids formed with LXA4-treated hPSCs expressed significantly lower levels of Coll compared to EtOH-treated hPSCs (Fig. $5 \mathrm{H}$, I). These data signify that LXA4 intervenes into the crosstalk between hPSCs and tumor cells by inhibiting the activation of hPSCs.

\subsubsection{LXA4 inhibits the growth of hPSC/Panc-1 3D- heterospheroid}

To investigate whether LXA4 also exerts an inhibitory effect on hPSC/Panc-1 3D-heterospheroid growth, we treated the established spheroids (4 days) with either EtOH or LXA4 and followed their growth. Similar to an in vivo tumor model, this 3D model involved the treatment after tumor a formation. The spheroids growth was observed over a time period of 12 days. A significant difference in spheroid growth between the LXA4 and control treated group was observed 2 days after the start of the treatment (Fig. 6A, B). Furthermore, the control treated spheroids showed newly formed areas of proliferating cells on their surface, which was not observed in LXA4 treated spheroids (Fig. 6 A). In addition, we examined the effect of LXA4 on the spheroids solely consisting of Panc-1 cells to investigate if LXA4 also inhibits the Panc-1 3D-spheroid growth (Fig. $6 \mathrm{C}$ ). No significant decrease in spheroid growth could be observed in the LXA4-treated in comparison to the control Panc-1 3D-spheroids (Fig. 6 C). 
A

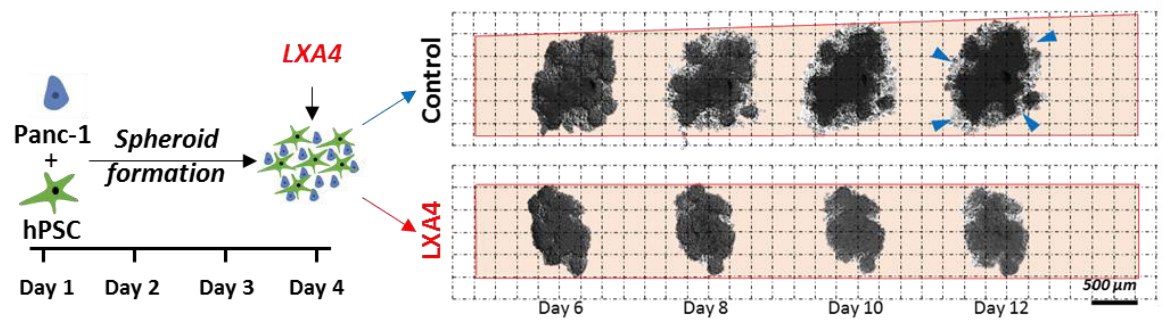

B

E
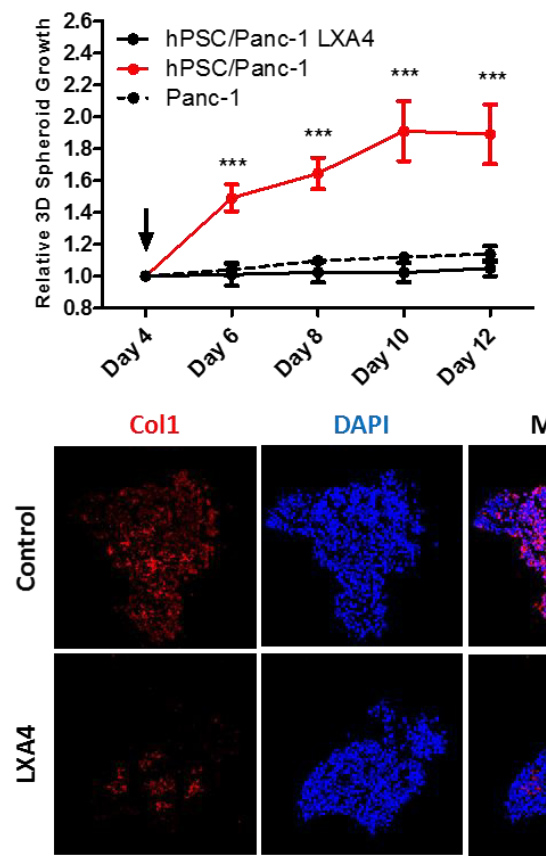

DAPI

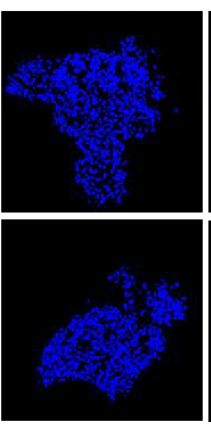

C

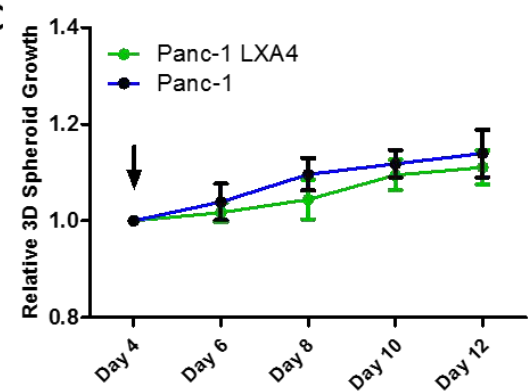

$\mathbf{F}$
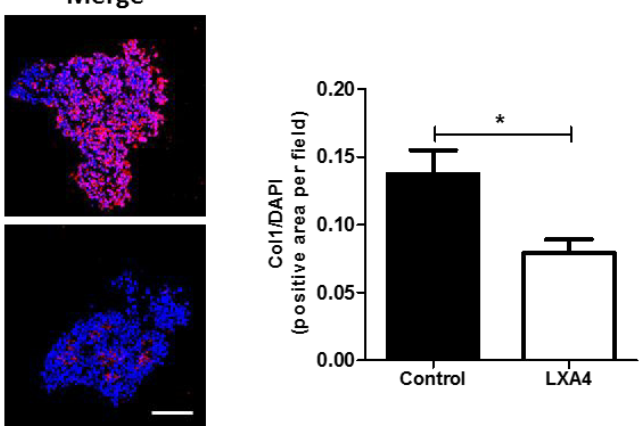

Fig. 6. Effect of LXA4 on hPSC/Panc-1 spheroid growth. Representative images (A) and quantification (B) of hPSC/Panc-1 3D-heterospheroid growth after treatment with LXA4 or control and Panc-1 3D-heterospheroids 4 days after initiation of the experiment. Black arrow indicates treatment with LXA4 or control, blue arrows indicate proliferating cells. Growth of Panc-1 3D-spheroids after treatment with LXA4 compared to control treated spheroids $(C)$. Immunofluorescent staining $(E)$ and quantification for the expression of Coll $(F)$ and nuclei staining $(D A P I)(F)$ in $6 \mu$ m thick section of control and LXA4 treated 3D-heterospheroids after 12 days of spheroid formation. Control contains $0.001 \%$ ethanol. Data represent mean + SEM for at least 3 independent experiments. Statistical analysis was performed by two-tailed unpaired student t-test. ${ }^{*} p$ $<0.05, * * p<0.01, * * * p<0.001$.

To examine the effect of LXA4 on the activation of hPSCs, we performed immunofluorescent staining on 3D-heterospheroids at day 12. Interestingly, significantly decreased levels of Col1 (Fig. 6 E, F) were found in LXA4-treated 
compared to EtOH-treated 3D-hetrospheroids. These data indicate that the reduction in the growth of the 3D-heterospheroids is due to inhibition of hPSC activation and its tumor-inducing effect but not due to a direct effect on the tumor cells.

\subsubsection{LXA4 inhibits in vivo subcutaneous tumor growth and collagen deposition in mice}

To investigate the effect of LXA4 on in vivo tumor growth we co-injected hPSCs and Panc-1 into the flank of immunodeficient mice (Fig. 7A).

A

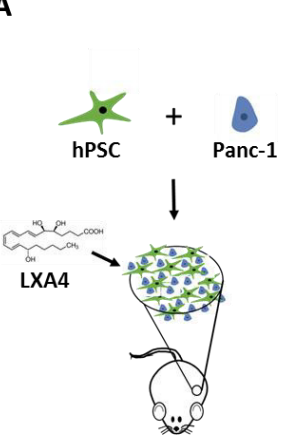

D

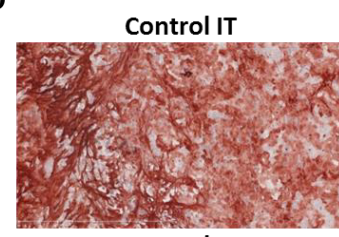

Control IP

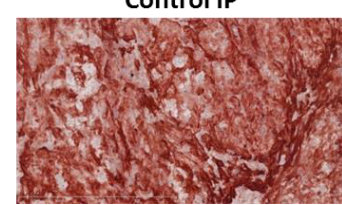

B

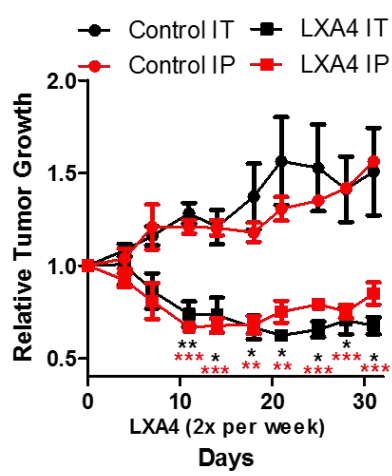

LXA4 IT

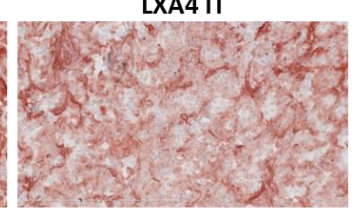

LXA4 IP

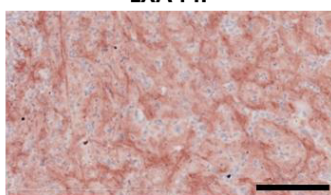

C

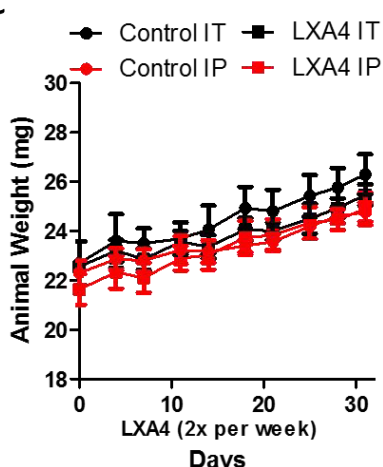

$\mathbf{E}$

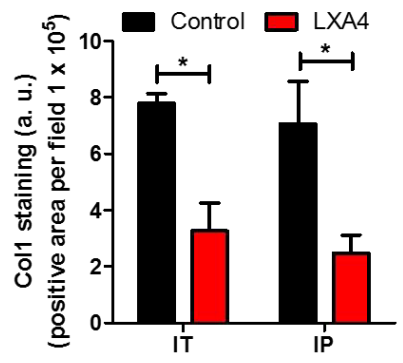

Fig. 7. Effect of LXA4-treatment on in vivo tumor growth and Col1 expression of Panc-1/PSC subcutaneous tumors in mice. Schematic representation of the co-injection (Panc-1 and hPSCs) subcutaneous tumor model (A). The effect of intra tumoral (IT) and intra peritoneal (IP) injection of LXA4 on the tumor growth of co-injection (Panc-1 + PSCs) subcutaneous xenografts in mice (B). Arrow indicate times of injection. The effect of intra tumoral (IT) and intra peritoneal (IP) injection of LXA4 on the body weight of co-injection (Panc-1 + PSCs) subcutaneous xenograft bearing mice $(C)$. Immunostaining $(D)$ and quantification $(E)$ of staining for Coll in tumors treated IT and IP with LXA4. Scale bar $=200 \mu \mathrm{m}$. Control contains $0.001 \%$ ethanol. Data represent 
mean $\pm S E M$ for at least 3 independent experiments. $* p<0.05, * * p<0.01, * * * p<$ 0.001 .

These tumors are rich in $\alpha$-SMA and thereby mimic the microenvironment of human PDAC patients (Supplementary Fig. 5). After the tumors were established, we treated the mice with either intratumoral (IT, $4 \mu \mathrm{g} / \mathrm{kg}$ ) or intraperitoneal (IP, $40 \mu \mathrm{g} / \mathrm{kg}$ ) injections of LXA4 or EtOH, twice a week (Fig. 7 B). Both, IT and IP injections of LXA4 significantly inhibited the tumor growth compared to control animals, immediately after the treatment (Fig. 7 B). The difference in tumor size of EtOH-treated and LXA4-treated animals stayed significant until the mice were sacrificed on day 31 after the first injection (Fig. 7 B). LXA-4 treatment showed no significant effects on the body weight of subcutaneous tumor bearing mice (Fig. 7 C). Immunofluorescent staining showed a significant reduction $(\sim 60 \%)$ of Coll in both IT and IP treated tumors compared to control tumors (Fig. 7 D, E). Additional stainings for the CAF marker ( $\alpha$-SMA), blood vessel marker (endothelial marker, CD31) did not show significant differences between $\mathrm{EtOH}$ - and LXA4-treated tumors, but there was a trend showing the reduction of these markers with LXA4 treatment (Supplementary Fig. 4).

\subsection{Discussion}

The abundant desmoplastic reaction in PDAC, also known as pancreatic tumor stroma, plays an important role in cancer progression, invasion, metastasis and response to therapy ${ }^{4}$. Reprogramming of the tumor stroma by dampening the function of hPSCs would be an interesting approach to gain therapeutic benefits for the treatment of cancer. In earlier studies, a natural biomolecule vitamin $\mathrm{D}$ has been shown to reverse hPSC activation to improve drug delivery efficiency and prolong survival in animals ${ }^{5}$. In this study, we explored the therapeutic potential of a highly potent endogenous lipid mediator, LXA4, for the treatment of pancreatic tumors. We showed that LXA4 inhibited the activation of hPSCs into CAF-like myofibroblasts in vitro and thereby inhibited hPSC-induced tumor-promoting effects. In our novel 3D-heterospheroidal co-cultures, LXA4 could inhibit the activation of hPSCs within the 3D microenvironment and reduced spheroid growth. Finally, we showed the therapeutic efficacy of LXA4 in vivo in a co-injection tumor model. This study highlights LXA4 as an interesting biological to be developed for the treatment of pancreatic cancer.

Human PSCs are regarded as the main source of CAFs in the stroma of pancreatic cancer ${ }^{12}$ and as response to activation with TGF- $\beta$ they acquire a CAF-like 
myofibroblast phenotype, as shown previously by us and others ${ }^{29-31}$. LXA4 a highly potent endogenous lipid is known for its anti-inflammatory function and has been shown to inhibit fibrosis in renal mesangial cells, human lung (myo)fibroblasts and experimental renal fibrosis ${ }^{13,23,24}$. Here we show that the LXA4-specific surface receptor FPR2 is expressed in hPSCs and highly upregulated in myofibroblast-like activated hPSCs. This result demonstrates that activated hPSCs present a valid target for treatment with LXA4. Our work illustrates for the first time that LXA4 treatment reduces the expression of fibrosis related myofibroblast markers $\alpha$-SMA and Col1 in TGF- $\beta$ activated hPSCs in vitro, which was accompanied by a reversal of the elongated, stretched myofibroblast-like phenotype of those cells into their normal phenotype. In addition, LXA4 treatment significantly reduced the TGF- $\beta$-induced expression of $\alpha$-SMA, Col1 $\alpha 1$, Col3 $\alpha 1$, PDGFR $\beta$, MMP2, Wnt2B, NSDHL, CXCL12, IL, 6, CTGF, IDI1 and INSIG1, all markers which were previously found to be induced in activated PSCs by transcriptome analysis 5,32 . Of special significance are the LXA4 targets Col1 $\alpha 1$ and Col3 $\alpha 1$ of the extracellular matrix, limiting chemotherapeutic activity by blocking drug delivery ${ }^{33,34}$, and growth factors CTGF and CXCL12, known to potentiate the tumor response to gemcitabine ${ }^{35}$ and restore T-cell response ${ }^{36}$, respectively. The effects of LXA4 on transcriptional changes in hPSCs were supported by a reduction in hPSC migration upon LXA4 treatment, confirming the significance of LXA4 in controlling hPSC's phenotypic behaviour. These findings illustrate that LXA4 treatment remodels activated hPSCs into their quiescent state by transcriptional remodelling and thereby inhibits the capacity of those cells to support pancreatic tumor growth. Upon activation of hPSCs with TGF- $\beta$ the Smad 2/3 signalling pathway is activated, resulting in phosphorylation of the Smad $2 / 3$ protein ${ }^{37}$. This data demonstrates that TGF- $\beta$ induced transcriptional and phenotypic changes were controlled by LXA4-induced inhibition of Smad 2/3 phosphorylation, which has earlier been observed by Börgeson et al. in renal rat fibroblasts ${ }^{38}$. Previous studies have demonstrated that hPSCs can induce tumorpromoting paracrine effects by secreting mutagenic factors such as growth factors and cytokines to induce tumor progression, invasion and metastasis ${ }^{39,40}$. Interestingly, our study shows a modulation of the secretory phenotype of hPSCs after treatment with LXA4, which attenuated hPSC-mediated cell growth and migration of Panc-1 and AsPc-1 tumor cells. These results are in line with previous studies in which hPSCs paracrine effects on tumor cells were reduced by the means of microRNA ${ }^{29}$. The inhibitory effect of LXA4 on hPSC activation and hPSC induced tumor promoting effects were not only limited to 2D cell 
cultures but also inhibited the formation, growth and ECM-deposition of 3Dheterospheroids formed from hPSCs and Panc-1. These 3D-heterospheroids provide tumor-like characteristics such as a dense, hypoxic core, ECM production and direct crosstalk between stromal and cancer cells. In line with our in vitro data, administration of LXA4 via IT or IP injection into hPSC/Panc1 subcutaneous tumors in mice reduced Col1 deposition, the major ECM protein within the pancreatic tumor stroma. Finally, the reduced ECM deposition within these tumors, resulted in attenuated tumor progression. In this context, it is important to point out, that ECM degradation resulting in improved intratumoral blood flow and drug delivery has shown survival benefits in pancreatic cancer ${ }^{6,41}$. Staining for the CAF marker $\alpha$ SMA was reduced in the tumors of mice which obtained IP injections of LXA4 but not in IT treated tumors (Supplementary Figure $4 \mathrm{~A}$ ), indicating normalization of the CAF phenotype. The reduced $\alpha$ SMA expression in only IP treated mice points out towards the additional systemic effect of LXA4. However, since the local IT injection reduced the tumor growth, it is clear that the tumor-inhibitory effect of LXA4 is due to a direct effect on the tumor stroma. In addition, LXA4 reduced angiogenesis, a key tumor-promoting mechanism, in tumors of both IT and IP LXA4-treated animals, indicated by the endothelial marker CD31 (Supplementary Figure 4 B).

In conclusion, this study demonstrated for the first time that LXA4 is capable to attenuate tumor growth by inhibiting PSC-induced desmoplasia in pancreatic tumors, by reversing hPSC activation into CAF and modulating hPSC induced tumor promoting effects. Therefore, LXA4 is a potential candidate to be evaluated for the treatment of pancreatic tumor and for its potential to increase the efficacy of chemo- and radiotherapy.

\subsection{Financial supports}

The study was supported by the Swedish Research Council, Stockholm (project no. 2011-5389) and the MIRA Institue for Biomedical Technology and Technical Medicine, University of Twente, The Netherlands

\subsection{Conflicts of interest}

J Prakash is a founder and stakeholder of ScarTec Therapeutics BV. Enschede. The authors have no other relevant affiliations or financial involvement with any organization or entity with a financial interest in or financial conflict with the subject matter or materials discussed in the manuscript apart from those disclosed. 


\subsection{References}

1 Siegel, R. L., Miller, K. D. \& Jemal, A. Cancer statistics, 2016. CA: $a$ cancer journal for clinicians 66, 7-30, doi:10.3322/caac.21332 (2016).

2 Conroy, T. et al. FOLFIRINOX versus gemcitabine for metastatic pancreatic cancer. The New England journal of medicine 364, 18171825, doi:10.1056/NEJMoa1011923 (2011).

3 Von Hoff, D. D. et al. Increased survival in pancreatic cancer with nabpaclitaxel plus gemcitabine. The New England journal of medicine 369, 1691-1703, doi:10.1056/NEJMoa1304369 (2013).

4 Xie, D. \& Xie, K. Pancreatic cancer stromal biology and therapy. Genes \& diseases 2, 133-143, doi:10.1016/j.gendis.2015.01.002 (2015).

5 Sherman, M. H. et al. Vitamin D receptor-mediated stromal reprogramming suppresses pancreatitis and enhances pancreatic cancer therapy. Cell 159, 80-93, doi:10.1016/j.cell.2014.08.007 (2014).

6 Provenzano, P. P. et al. Enzymatic targeting of the stroma ablates physical barriers to treatment of pancreatic ductal adenocarcinoma. Cancer Cell 21, 418-429, doi:10.1016/j.ccr.2012.01.007 (2012).

7 Ozdemir, B. C. et al. Depletion of carcinoma-associated fibroblasts and fibrosis induces immunosuppression and accelerates pancreas cancer with reduced survival. Cancer cell 25, 719-734, doi:10.1016/j.ccr.2014.04.005 (2014).

8 Prakash, J. Cancer-Associated Fibroblasts: Perspectives in Cancer Therapy. Trends in cancer 2, 277-279, doi:10.1016/j.trecan.2016.04.005 (2016).

9 Shields, M. A., Dangi-Garimella, S., Redig, A. J. \& Munshi, H. G. Biochemical role of the collagen-rich tumour microenvironment in pancreatic cancer progression. The Biochemical journal 441, 541-552, doi:10.1042/BJ20111240 (2012).

10 Kuninty, P. R., Schnittert, J., Storm, G. \& Prakash, J. MicroRNA Targeting to Modulate Tumor Microenvironment. Front Oncol 6, 3, doi:10.3389/fonc.2016.00003 (2016).

11 Habisch, H., Zhou, S., Siech, M. \& Bachem, M. G. Interaction of stellate cells with pancreatic carcinoma cells. Cancers (Basel) 2, 1661-1682, doi:10.3390/cancers2031661 (2010).

12 Apte, M. V., Wilson, J. S., Lugea, A. \& Pandol, S. J. A starring role for stellate cells in the pancreatic cancer microenvironment. Gastroenterology 144, 1210-1219, doi:10.1053/j.gastro.2012.11.037 (2013). 
13 Roach, K. M., Feghali-Bostwick, C. A., Amrani, Y. \& Bradding, P. Lipoxin A4 Attenuates Constitutive and TGF-beta1-Dependent Profibrotic Activity in Human Lung Myofibroblasts. J Immunol 195, 2852-2860, doi:10.4049/jimmunol.1500936 (2015).

14 Chandrasekharan, J. A. \& Sharma-Walia, N. Lipoxins: nature's way to resolve inflammation. $J$ Inflamm Res 8, 181-192, doi:10.2147/JIR.S90380 (2015).

15 Serhan, C. N. \& Savill, J. Resolution of inflammation: the beginning programs the end. Nat Immunol 6, 1191-1197, doi:10.1038/ni1276 (2005).

16 Fierro, I. M. \& Serhan, C. N. Mechanisms in anti-inflammation and resolution: the role of lipoxins and aspirin-triggered lipoxins. Braz J Med Biol Res 34, 555-566 (2001).

17 Serhan, C. N., Hamberg, M. \& Samuelsson, B. Lipoxins: novel series of biologically active compounds formed from arachidonic acid in human leukocytes. Proc Natl Acad Sci U S A 81, 5335-5339 (1984).

18 Serhan, C. N. \& Sheppard, K. A. Lipoxin formation during human neutrophil-platelet interactions. Evidence for the transformation of leukotriene A4 by platelet 12-lipoxygenase in vitro. J Clin Invest $\mathbf{8 5}$, 772-780, doi:10.1172/JCI114503 (1990).

19 Claria, J. \& Serhan, C. N. Aspirin triggers previously undescribed bioactive eicosanoids by human endothelial cell-leukocyte interactions. Proc Natl Acad Sci U S A 92, 9475-9479 (1995).

20 Serhan, C. N., Chiang, N. \& Van Dyke, T. E. Resolving inflammation: dual anti-inflammatory and pro-resolution lipid mediators. Nat Rev Immunol 8, 349-361, doi:10.1038/nri2294 (2008).

21 Serhan, C. N. Pro-resolving lipid mediators are leads for resolution physiology. Nature 510, 92-101, doi:10.1038/nature13479 (2014).

22 Rodgers, K., McMahon, B., Mitchell, D., Sadlier, D. \& Godson, C. Lipoxin A4 modifies platelet-derived growth factor-induced pro-fibrotic gene expression in human renal mesangial cells. Am J Pathol 167, 683694, doi:10.1016/S0002-9440(10)62043-3 (2005).

23 Mitchell, D. et al. Lipoxins inhibit Akt/PKB activation and cell cycle progression in human mesangial cells. The American journal of pathology 164, 937-946, doi:10.1016/S0002-9440(10)63181-1 (2004).

24 Borgeson, E. et al. Lipoxin $\mathrm{A}(4)$ and benzo-lipoxin $\mathrm{A}(4)$ attenuate experimental renal fibrosis. FASEB journal : official publication of the 
Federation of American Societies for Experimental Biology 25, 29672979, doi:10.1096/fj.11-185017 (2011).

25 Wu, S. H., Zhang, Y. M., Tao, H. X. \& Dong, L. Lipoxin A(4) inhibits transition of epithelial to mesenchymal cells in proximal tubules. American journal of nephrology 32, 122-136, doi:10.1159/000315121 (2010).

26 Wu, S. H., Wu, X. H., Lu, C., Dong, L. \& Chen, Z. Q. Lipoxin A4 inhibits proliferation of human lung fibroblasts induced by connective tissue growth factor. American journal of respiratory cell and molecular biology 34, 65-72, doi:10.1165/rcmb.2005-0184OC (2006).

27 Bansal, R. et al. PEGylation improves pharmacokinetic profile, liver uptake and efficacy of Interferon gamma in liver fibrosis. Journal of controlled release : official journal of the Controlled Release Society 154, 233-240, doi:10.1016/j.jconrel.2011.05.027 (2011).

28 Schnittert, J. et al. Anti-microRNA targeting using peptide-based nanocomplexes to inhibit differentiation of human pancreatic stellate cells. Nanomedicine, doi:10.2217/nnm-2017-0054 (2017).

29 Kuninty, P. R. et al. MicroRNA-199a and -214 as potential therapeutic targets in pancreatic stellate cells in pancreatic tumor. Oncotarget 7, 16396-16408, doi:10.18632/oncotarget.7651 (2016).

30 Costa-Silva, B. et al. Pancreatic cancer exosomes initiate pre-metastatic niche formation in the liver. Nature cell biology 17, 816-826, doi:10.1038/ncb3169 (2015).

31 Sarper, M., Cortes, E., Lieberthal, T. J. \& Del Rio Hernandez, A. ATRA modulates mechanical activation of TGF-beta by pancreatic stellate cells. Sci Rep 6, 27639, doi:10.1038/srep27639 (2016).

32 Kalluri, R. The biology and function of fibroblasts in cancer. Nature reviews. Cancer 16, 582-598, doi:10.1038/nrc.2016.73 (2016).

33 Minchinton, A. I. \& Tannock, I. F. Drug penetration in solid tumours. Nature reviews. Cancer 6, 583-592, doi:10.1038/nrc1893 (2006).

34 Netti, P. A., Berk, D. A., Swartz, M. A., Grodzinsky, A. J. \& Jain, R. K. Role of extracellular matrix assembly in interstitial transport in solid tumors. Cancer research 60, 2497-2503 (2000).

35 Neesse, A. et al. CTGF antagonism with mAb FG-3019 enhances chemotherapy response without increasing drug delivery in murine ductal pancreas cancer. Proc. Natl. Acad. Sci. U. S. A. 110, 1232512330, doi:10.1073/pnas.1300415110 (2013). 
36 Ding, N. et al. A vitamin D receptor/SMAD genomic circuit gates hepatic fibrotic response. Cell 153, 601-613, doi:10.1016/j.cell.2013.03.028 (2013).

37 Neuzillet, C. et al. Perspectives of TGF-beta inhibition in pancreatic and hepatocellular carcinomas. Oncotarget 5, 78-94, doi:10.18632/oncotarget.1569 (2014).

38 Borgeson, E. et al. Lipoxin $\mathrm{A}(4)$ and benzo-lipoxin $\mathrm{A}(4)$ attenuate experimental renal fibrosis. Faseb Journal 25, 2967-2979, doi:10.1096/fj.11-185017 (2011).

39 Apte, M. V., Wilson, J. S., Lugea, A. \& Pandol, S. J. A Starring Role for Stellate Cells in the Pancreatic Cancer Microenvironment. Gastroenterology 144, 1210-1219, doi:10.1053/j.gastro.2012.11.037 (2013).

40 Hwang, R. F. et al. Cancer-associated stromal fibroblasts promote pancreatic tumor progression. Cancer research 68, 918-926, doi:10.1158/0008-5472.CAN-07-5714 (2008).

41 Jacobetz, M. A. et al. Hyaluronan impairs vascular function and drug delivery in a mouse model of pancreatic cancer. Gut 62, 112-120, doi:10.1136/gutjnl-2012-302529 (2013). 


\subsection{Supplementary Data}

A

\section{Smad 2/3 \\ pSmad 2/3}
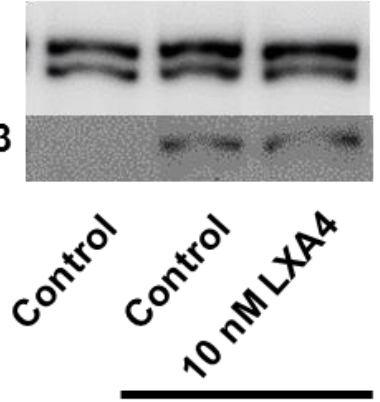

TGF- $\beta$
B

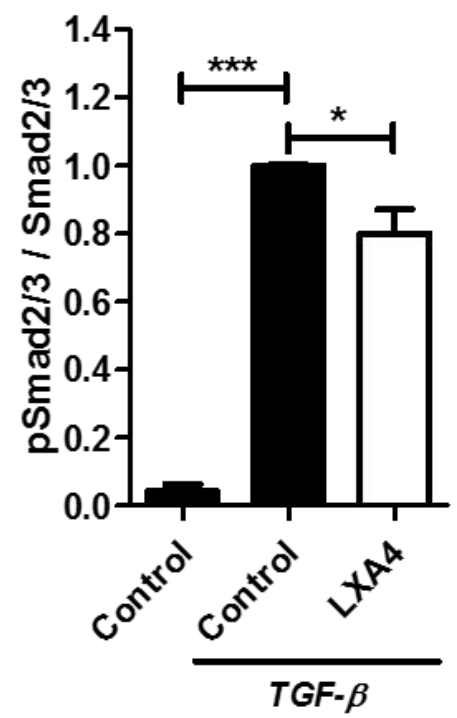

Supplementary Figure 1. Western Blot showing Smad 2/3 and pSmad 2/3 levels in hPSCs, TGF- $\beta$ activated hPSCs treated with control or $10 \mathrm{nM} \mathrm{LXA4} \mathrm{(A).} \mathrm{Quantification}$ showing the ratio of pSmad 2/3 / Smad 2/3 (B). Control contains $0.001 \%$ ethanol. Data represent mean + SEM for at least 3 independent experiments. Statistical analysis was performed by two-tailed unpaired student t-test. $* p<0.05, * * * 0.001$. 
A

B

$\begin{array}{lllll}\text { TGF- } \beta & - & - & + & + \\ \text { LXA4 } & - & - & - & + \\ \text { hPSC } & - & + & + & +\end{array}$

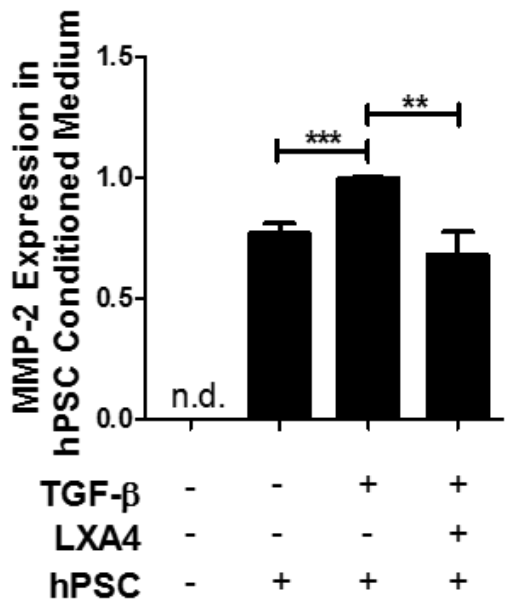

Supplementary Figure 2. Western Blot showing MMP-2 in control medium, hPSC conditioned medium, conditioned medium from TGF- $\beta$ activated hPSCs and conditioned medium from TGF- $\beta$ activated hPSCs treated $10 \mathrm{nM} \mathrm{LXA4} \mathrm{(A).} \mathrm{Quantification} \mathrm{showing}$ the expression of MMP-2 (B). Controls contains $0.001 \%$ ethanol. Data represent mean + SEM for at least 3 independent experiments. Statistical analysis was performed by two-tailed unpaired student $t$-test. $* * p<0.01, * * *<0.001$.

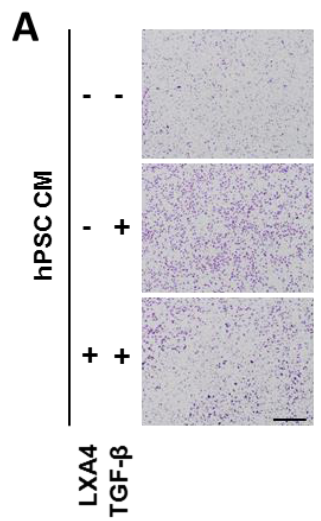

B

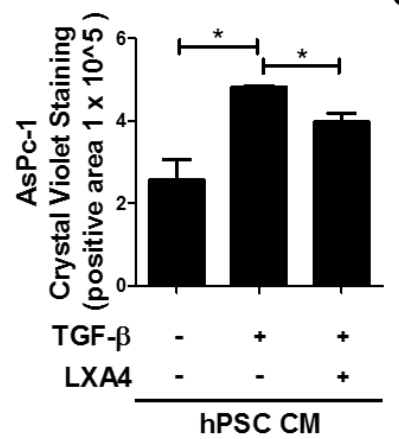

C

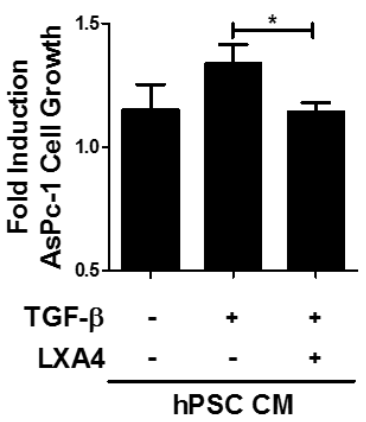

Supplementary Figure 3. Representative images (A) and quantification (B) of AsPc-1 tumor cell migration after 24 hours of incubation with condition medium collected from untreated, control-treated TGF- $\beta$ activated hPSCs and 10 nM LXA4-treated TGF- $\beta$ activated hPSCs. Scale bar $=400 \mu \mathrm{m}$. AsPc-1 tumor cell growth after 48 hours of incubation with condition medium collected from untreated, control-treated TGF- $\beta$ activated hPSCs and 10 nM LXA4-treated TGF- $\beta$ activated hPSCs (C). Controls 
contains $0.001 \%$ ethanol. Data represent mean + SEM for at least 3 independent experiments. Statistical analysis was performed by two-tailed unpaired student t-test. $*<0.05, * * p<0.01, * * *<0.001$.

A

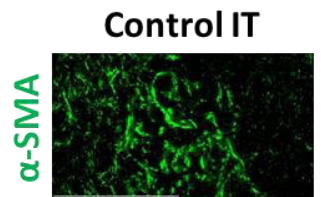

LXA4 IT
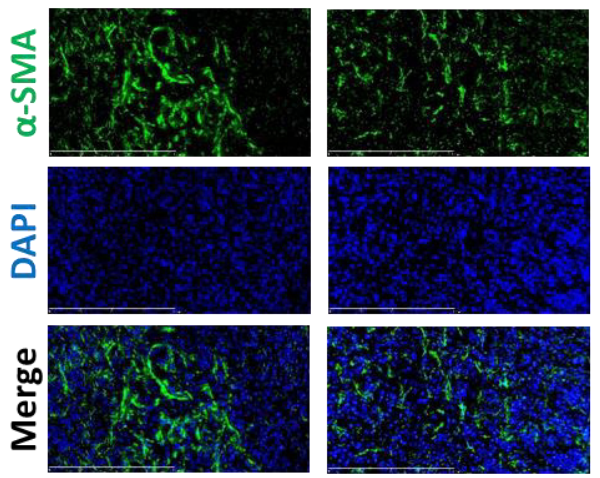

B

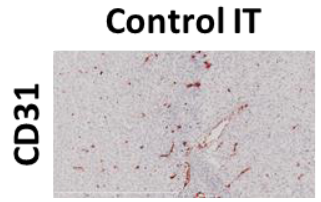

LXA4 IT

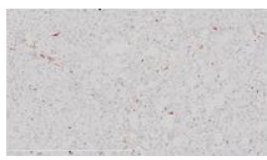

Control IP
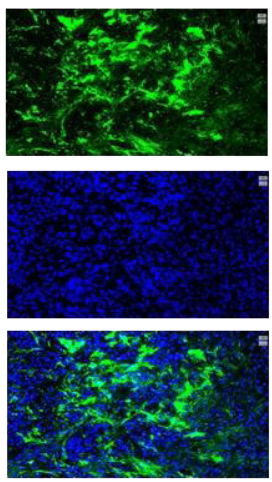

Control IP

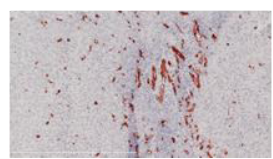

LXA4 IP
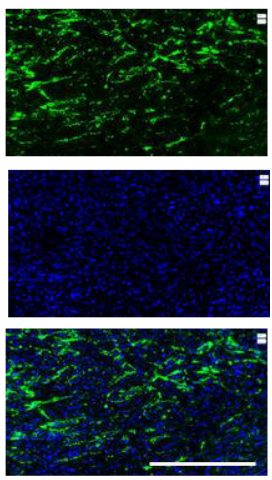

LXA4 IP

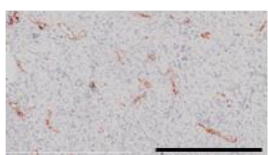

Supplementary Figure 4. Immunostaining and quantification of staining for $\alpha-S M A$ (A) and CD-31 (B) in tumors treated IT and IP with LXA4. Scale bar $=200 \mu \mathrm{m}$. Control contains $0.001 \%$ ethanol. Scale bar $=400 \mu \mathrm{m}$.

Human PDAC

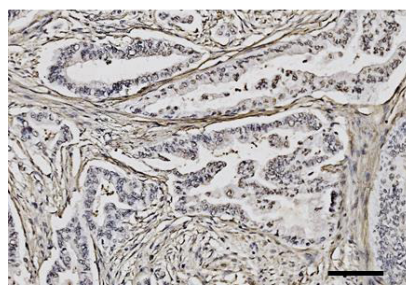

hPSC + Panc-1

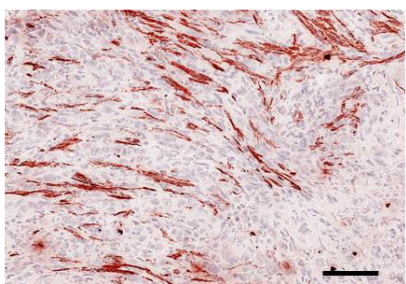

Panc-1

\section{Panc-1}

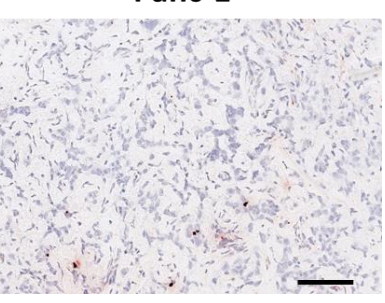

Supplementary Figure 5. Immunostaining for $\alpha-S M A$ in human PDAC and Panc-1 and Panc-1+hPSC subcutaneous mouse tumors. Scale bar $=100 \mu \mathrm{m}$.

Supplementary table 1. Primary and secondary antibodies used for western blot.

\begin{tabular}{|l|l|l|}
\hline Antibody & Source & Dilution \\
\hline Rabbit polyclonal anti-FPR2 & Sigma Aldrich & $1: 100$ \\
\hline
\end{tabular}




\begin{tabular}{|l|l|l|}
\hline Mouse anti- $\alpha$-SMA & Sigma Aldrich & $1: 200$ \\
\hline Rabbit monoclonal anti-Smad 2/3 & Cell Signaling & $1: 1000$ \\
\hline Rabbit monoclonal anti-pSmad 2/3 & Cell Signaling & $1: 1000$ \\
\hline Mouse monoclonal anti- $\beta$-actin & Sigma Aldrich & $1: 10000$ \\
\hline HRP-conjugated goat anti-rabbit IgG & DAKO & $1: 2000$ \\
\hline HRP-conjugated rabbit anti-goat IgG & DAKO & $1: 2000$ \\
\hline HRP-conjugated rabbit anti-mouse IgG & DAKO & $1: 1000$ \\
\hline
\end{tabular}

Supplementary Table 2. Primary and secondary antibodies used for immunohistochemical staining of cells, spheroids and tumor sections.

\begin{tabular}{|l|l|l|}
\hline Antibody & Source & Dilution \\
\hline Rabbit monoclonal anti-Smad2/3 & Cell Signaling & $1: 1500$ \\
\hline Rabbit monoclonal anti-pSmad2/3 & Cell Signaling & $1: 200$ \\
\hline Goat polyclonal anti-Collagen1 & Southern Biotech & $1: 250$ \\
\hline Goat polyclonal anti-PECAM-1 (CD31) & Southern Biotech & $1: 50$ \\
\hline Mouse monoclonal anti- $\alpha$ SMA & Sigma Aldrich & $1: 400$ \\
\hline Rabbit anti- $\alpha$ SMA & Thermo Fisher Scientific & $1: 100$ \\
\hline Rabbit monoclonal anti-MMP-2 & Cell Signaling & $1: 1000$ \\
\hline HRP-conjugated rabbit anti-goat IgG & DAKO & $1: 100$ \\
\hline HRP-conjugated rabbit anti-mouse IgG & DAKO & $1: 1000$ \\
\hline Alexa Fluor 594 labelled donkey anti-goat & Life-Technologies & $1: 100$ \\
\hline Alexa Fluor 488 labelled donkey anti-rabbit & Life-Technologies & $1: 100$ \\
\hline Alexa Fluor 488 labelled donkey anti-mouse & Life-Technologies & $1: 100$ \\
\hline
\end{tabular}




\section{Chapter 5 - Integrins in wound healing, fibrosis and tumor stroma: High potential targets for therapeutics and drug delivery}

Jonas Schnittert ${ }^{\mathrm{a}}$, Ruchi Bansal ${ }^{\mathrm{a}}$, Gert Storm ${ }^{\mathrm{a}, \mathrm{b}}$, Jai Prakash ${ }^{\mathrm{a}, \mathrm{b}, \S}$

${ }^{a}$ Section - Targeted Therapeutics, Department of Biomaterials Science and Technology, University of Twente, Enschede, The Netherlands. ${ }^{\mathrm{b}}$ Department of Pharmaceutics, Utrecht University, Utrecht, The Netherlands. ${ }^{b}$ ScarTec Therapeutics BV. Enschede, The Netherlands.

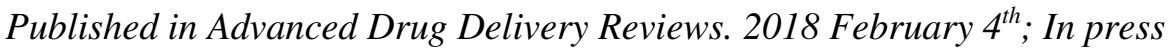

\section{Abstract}

Wound healing is a complex process, which ultimately leads to fibrosis if not repaired well. Pathologically very similar to fibrosis is the tumor stroma, found in several solid tumors which are regarded as wounds that do not heal. Integrins are heterodimeric surface receptors which control various physiological cellular functions. Additionally, integrins also sense ECM-induced extracellular changes during pathological events, leading to cellular responses, which influence ECM remodeling. The purpose and scope of this review is to introduce integrins as key targets for therapeutics and drug delivery within the scope of wound healing, fibrosis and the tumor stroma. This review provides a general introduction to the biology of integrins including their types, ligands, means of signaling and interaction with growth factor receptors. Furthermore, we highlight integrins as key targets for therapeutics and drug delivery, based on their biological role, expression pattern within human tissues and at cellular level. Next, therapeutic approaches targeting integrins, with a focus on clinical studies, and targeted drug delivery strategies based on ligands are described. 


\section{Graphical abstract}

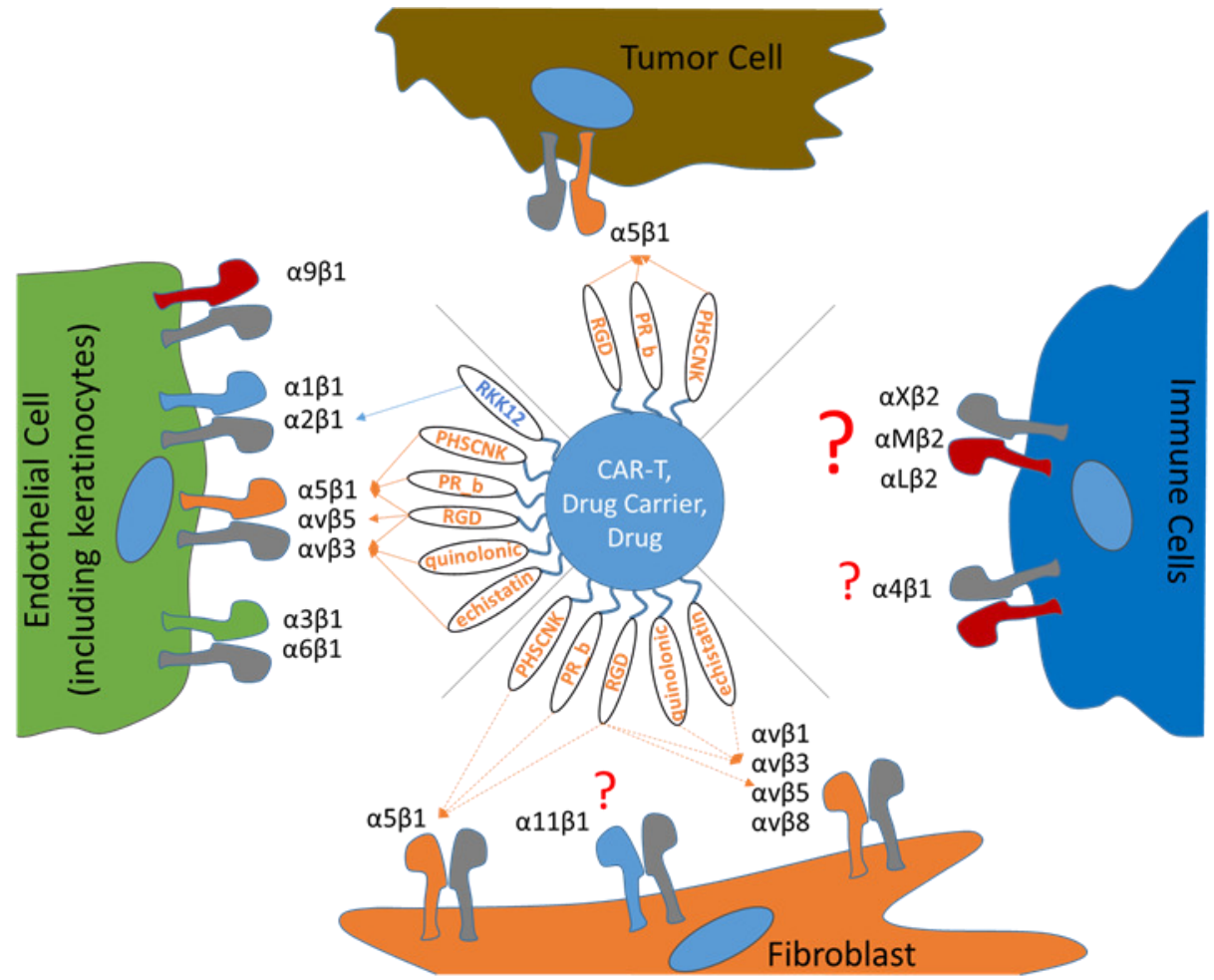

Existing integrin drug targeting ligands, applied for the modification of drug molecules, drug carriers and CAR-T cells (CAR-T), in the context of wound healing, fibrosis or tumor stroma and their respective integrin targets. Dashed lines show potential integrin targets for existing ligands (PHSCNK, PR_b, RGD, RKK12, quinolonic, echistatin) that have not yet been used for targeting the respective cell line. In addition to already targeted integrins, integrins that have not yet been targeted but play a role in the pathology of wound healing, fibrosis or tumor stroma are presented. 


\subsection{Introduction}

Wound healing is a complex process, which if not repaired leads to scar formation so-called fibrosis. Fibrosis, a hallmark of an excessive extracellular matrix (ECM) deposition, accounts for about $45 \%$ of lethalities in the modern world ${ }^{1}$. In general, fibrotic diseases are caused by chronic tissue injury, resulting in chronic inflammation and fibrosis which leads to destruction of the normal tissue architecture and ultimately organ failure. Currently, there are no effective treatment opportunities for tissue fibrosis and therefore there is a desperate need for effective anti-fibrotic therapies. In addition, tumors are regarded as "wounds that do not heal" due to extensive fibrosis within tumors. Fibrosis and tumor share a strikingly similar cellular and microenvironmental reactivity. Several tumor types undergo a fibrotic reaction so-called desmoplasia or tumor stroma. Tumor stroma has been shown to strongly support the tumor growth by many means and therefore these tumors are referred to as fibrosis-driven tumors ${ }^{2}$.

Tissue fibrogenesis is a complex process orchestrated by a bidirectional crosstalk between the different cell types including inflammatory cells, epithelial cells, myofibroblast and ECM in response of the wound healing process ${ }^{3}$. Cell fate within fibrotic tissues is profoundly affected by the highly dynamic environment of the pericellular ECM mainly produced by myofibroblasts ${ }^{4}$. During fibrosis, integrins, a family of transmembrane receptors, mediate various cell-matrix and cell-cell interactions. Integrins facilitate communication between the ECM, nonparenchymal cells including inflammatory cells, fibroblasts, and parenchymal cells, and by these interactions, integrins are directly involved in the initiation and progression of tissue fibrosis ${ }^{4}$. Therefore, integrins represent highly interesting therapeutic targets.

Within this review, we provide a general introduction of integrins and integrinmediated signaling, an overview of integrin expression in fibrosis-related cell types, and interaction between integrins and growth factor receptors. The next section describes the role of integrins in wound healing, fibrosis-driven tumor, tumor metastasis and fibrosis. Finally, the last two sections are focused on the novel therapies based on integrin inhibition, including clinical developments, and drug delivery strategies to target integrins. 


\subsection{Integrin receptors}

\subsubsection{Integrin heterodimers and ligand specificity}

Integrins are a family of heterodimeric cell surface receptors, each consisting of one $\alpha$ and one $\beta$ subunit. Overall, there are $18 \alpha$ and $8 \beta$ subunits that combine and form heterodimers with 24 different functional integrin receptors identified so far. Each integrin receptor specifically binds to one or more ligands. Their specific ligand binding ability enables cells to connect with its surrounding extracellular matrix (ECM), thereby enabling cell motility and invasion. Integrins possess a physical connection with the inside and the outside of a cell, which allows for bidirectional sensing of signals. With this mechanism, integrins ultimately control cytoskeleton organization, thereby directly affecting essential cellular functions such as cell adhesion, migration, proliferation, survival and differentiation ${ }^{5}$. The local expression pattern of both integrins and their ligands controls the response of a cell to its microenvironment, as every individual integrin heterodimer is capable to bind multiple ligands and also a ligand may bind to multiple integrin heterodimers ${ }^{5}$.

In addition to controlling a range of physiological functions, integrins also sense ECM-induced extracellular changes during pathological events such as fibrosis, cancer and wound healing, leading to cellular responses, which influence ECM remodeling ${ }^{5}$. Next to binding ECM components, integrins are also capable of participating in cell-cell adhesions, for which they bind to counter receptors on adjacent cells such as ADAMs (A Disintergins And Matrix metalloproteinases (MMPs)), thereby promoting matrix remodeling ${ }^{6}$; as well as immunoglobulintype receptors such as intracellular adhesion molecules (ICAMs) and vascular cell adhesion molecules (VCAMs) which are expressed on leukocytes and endothelial cells ${ }^{7}$.

Integrins can be classified into five different integrin subfamilies (Table 1) ${ }^{6}$. Integrin $\alpha 1 \beta 1, \alpha 2 \beta 1, \alpha 10 \beta 1, \alpha 11 \beta 1$, belong to the $\beta 1$ containing collagen receptors ${ }^{6}$. The integrins $\alpha 5 \beta 1, \alpha 8 \beta 1, \alpha v \beta 1, \alpha v \beta 3$, $\alpha v \beta 5, \alpha v \beta 6, \alpha v \beta 8$ and $\alpha \operatorname{Ilb} \beta 1$, belong to the RGD (arginine-glycine-aspartic acid)-binding integrins capable of binding to the ECM and plasma proteins such as fibronectin, vitronectin, fibrinogen and thrombospondin ${ }^{6,8}$. The integrins $\alpha 3 \beta 1, \alpha 6 \beta 1, \alpha 7 \beta 1$ and $\alpha 6 \beta 4$ are laminin receptors that mediate cell adhesion to the basement membranes of various tissues ${ }^{6}$. The $\alpha 4 \beta 1, \alpha 9 \beta 1, \alpha 4 \beta 7$ integrin family also binds to fibronectin but in a RGD-independent manner via the adhesive sequences EILDV and REDV manner ${ }^{6,8}$. Additionally, integrin $\alpha 4 \beta 1$ and $\alpha 4 \beta 7$ are also able counter 
receptors in other cells e.g. intercellular adhesion molecules ${ }^{6}$. Integrins $\alpha \mathrm{D} \beta 2$, $\alpha \mathrm{L} \beta 2, \alpha \mathrm{M} \beta 2, \alpha \mathrm{X} \beta 2$ and $\alpha \mathrm{E} \beta 2$ belong to the leukocyte integrin subgroup, binding to receptors such as intracellular adhesion molecules (ICAMs) and plasma proteins such as complement component $\mathrm{C} 3 \mathrm{~b}$ and $\mathrm{C} 4 \mathrm{~b}^{6}$.

Table 1. Integrin heterodimer subfamilies comprised of 24 integrin receptors and their respective ligands.

\begin{tabular}{|c|c|}
\hline Integrin receptor subfamilies & Integrin Type \\
\hline Collagen receptors & $\alpha 1 \beta 1, \alpha 2 \beta 1, \alpha 10 \beta 1, \alpha 11 \beta 1$ \\
\hline $\begin{array}{l}\text { Fibronectin, vitronectin, fibrinogen and } \\
\text { thrombospondin receptors (RGD binding) }\end{array}$ & $\begin{array}{l}\alpha 5 \beta 1, \alpha 8 \beta 1, \alpha v \beta 1, \alpha v \beta 3, \alpha v \beta 5, \alpha v \beta 6, \alpha v \beta 8, \\
\alpha \operatorname{IIb} \beta 1\end{array}$ \\
\hline Laminin receptors & $\alpha 3 \beta 1, \alpha 6 \beta 1, \alpha 7 \beta 1, \alpha 6 \beta 4$ \\
\hline Fibronectin receptors (non-RGD binding) & $\alpha 4 \beta 1, \alpha 9 \beta 1, \alpha 4 \beta 7$ \\
\hline Leukocyte receptors & $\begin{array}{l}\alpha \mathrm{D} \beta 2, \quad \alpha \mathrm{L} \beta 2, \quad \alpha \mathrm{M} \beta 2, \alpha \mathrm{X} \beta 2, \alpha \mathrm{E} \beta 2, \alpha 4 \beta 1, \\
\alpha 4 \beta 7\end{array}$ \\
\hline
\end{tabular}

\subsubsection{Integrin signaling}

During interactions of integrins with ligands of the surrounding ECM, integrins undergo a conformational change and cluster in the plane of the cell-membrane 8 . This change in conformation activates integrins to a high avidity state. Within this state, integrins recruit various signaling and adaptor molecules to form focal adhesions ${ }^{8}$. The composition of these focal adhesions varies dependent on whether these contacts are formed in a two-dimensional or three-dimensional environment ${ }^{9}$. While lacking kinase activity by themselves, clustered integrins are capable of recruiting and activating kinases such as focal adhesion kinase (FAK), Src family kinases (SFKs) and scaffold molecules such as p130CRKassociated substrate (p130CAS or BCAR1) ${ }^{8}$.

Next to activating kinases, integrins can also connect ECM to the actin cytoskeleton by recruiting proteins including talin, paxillin, $\alpha$-actinin, tensin and vinculin ${ }^{8}$. Furthermore, several scaffolding and signaling functions required for integrin-mediated effects on cell migration and survival are controlled by the integrin-linked kinase (ILK), PINCH and parvin. The ILK-PINCH-parvin (IPP) ternary complex functions as an essential signaling platform that regulates various scaffolding and signaling functions which enable integrin-mediated effects on cell migration and survival ${ }^{10}$. Moreover, integrins function in tumour 
cells might be regulated by integrin recruitment to microdomains by tetraspanin ${ }^{8}$. Regulation and activation of microdomains and other focal adhesion proteins influence cell adhesion and migration on the ECM. Many of these molecules are investigated as promising therapeutic targets ${ }^{8}$. In some cases, integrin function is based on its ligand binding affinity ${ }^{8}$. The affinity and activation of an integrin receptor can be induced by ligand-mediated integrin clustering or increased intracellular signaling driven by molecules such as GTPase RAP1A ${ }^{11}$. Therefore, signaling which is induced by oncogenes or growth factor receptors might influence integrin affinity and function ${ }^{8}$.

\subsubsection{Integrins and growth factor receptor interaction}

Integrins and growth factor receptors play an important role for signal integration

12. Crosstalk between integrins and growth factor receptors have been described for the TGF $\beta$ receptor, epidermal growth factor receptor (EGFR), Met receptor (belongs to HGFR superfamily, hepatocyte growth factor receptor), platelet derived growth factor receptor (PDGFR), insulin receptor and vascular endothelial growth factor receptor (VEGFR) ${ }^{12}$. There are various different classes of signal integration between different integrins and growth factor receptors ${ }^{13}$. In the following, we describe the five classes of signal coordination (i) concominant signaling, (ii) collaborative signaling, (iii) direct activation and (iv) amplification of signaling and (v) negative regulation (Figure 1) ${ }^{13}$.

\section{(i) Concomitant signaling:}

During concomitant signaling, integrins together with growth factor receptors signal independently to trigger the same signaling molecule (Figure 1). Pathways which are affected by concomitant signaling include Ras-MAPK (mitogenactivated protein kinase), PI3K-Akt (PI3K (phosphatidylinositol 3-kinase) and Akt (Protein Kinase B), and Rho ${ }^{12-15}$. As an example Akt can be activated by integrins and growth factor receptors in a PI3K-dependent manner via distinct mechanisms ${ }^{13}$. Phosphorylation of Akt at Ser473 and Thr308 and of Akt kinase acitivity is induced by integrin $\beta 1$ independenty of epithelial growth factor receptor signaling, while epithelial growth factor induces Akt activity via the Fak and Src independent of cell adhesion ${ }^{13}$. 


\section{(ii) Collaborative signaling:}

Collaborative signaling is very similar to concomitant signaling in the way that cells require integrin-mediated adhesion to proceed through the cell cycle and respond to growth factors since growth factor receptor signaling is inefficient in the absence of cell adhesion ${ }^{13}$. During collaborative signaling, integrin receptors create a permissive environment in which growth factor receptors can interact with downstream signaling molecules (Figure 1) ${ }^{16}$. The difference between concomitant and collaborative signaling is that during collaborative signaling the receptor signals are spatially and temporally controlled while in concomitant both integrin and growth factor receptor work independently ${ }^{13}$. An example for this type of signaling is that cells which only express Met in the absence of of integrin $\alpha 6 \beta 4$ no-longer respond to HGF showing the collaboration between integrin $\alpha 6 \beta 4$ and the Met receptor (HGFR) ${ }^{17}$.

\section{(iii) Direct activation:}

During direct activation of growth factor receptors, integrins induce growth factor phosphorylation in absence of the corresponding growth factor (Figure 1). This ligand-independent activation process has been shown for the growth factor receptors EGFR, Ron (Recepteur d'Origine nantais, member of HGFR superfamily), VEGFR, IGFR (insulin-like growth factor receptor) and PDGFR ${ }^{18-25}$. An example for direct activation is the ability of integrin $\alpha v \beta 3$ to activate VEGFR-2, IGFR-1 and PDGFR in a growth factor independent manner ${ }^{21-26}$. Additionally, in macrophages integrin $\beta 1$ is associated with Ron and the adhesion to collagen or fibronectin results in the phosphorylation of Ron in a Src-depentend manner and binding of integrin $\alpha 5 \beta 1$ by fibronectin induces activation of Met ${ }^{20,26}$.

\section{(iv) Amplification of signaling:}

Another form of crosstalk between integrins and growth factor receptors is amplification of signaling. It is based on the ability of growth factors to activate signaling by binding to their corresponding growth factor receptors which can than increase the expression of integrins ${ }^{13}$. This amplification process has for example been shown for HGF, increasing the expression of integrin $\alpha 2$ and $\alpha 3$ ${ }^{27,28}$, which could contribute to the amplification of Met signaling in response to HGF via the FAK-Src axis ${ }^{13}$. 


\section{(v) Negative regulation:}

As described above, integrins mostly function as positive regulators of growth factor receptor signaling. However, integrin interaction with the ECM can also inhibit growth factor receptor signaling through phosphatase activation and recruitment of, for example, $\mathrm{T}$ cell protein tyrosine phosphatase (TCPTP) (Figure 1) ${ }^{13}$. In renal fibrosis, it has been shown that Integrin $\alpha 1 \beta 1$ reduces Smad-dependent profibrotic signaling in kidney collecting duct derived cells by TCPTP-mediated dephosphorylation of TGF $\beta$ R2 (a variant of the TGF $\beta$ receptors II) ${ }^{29}$.

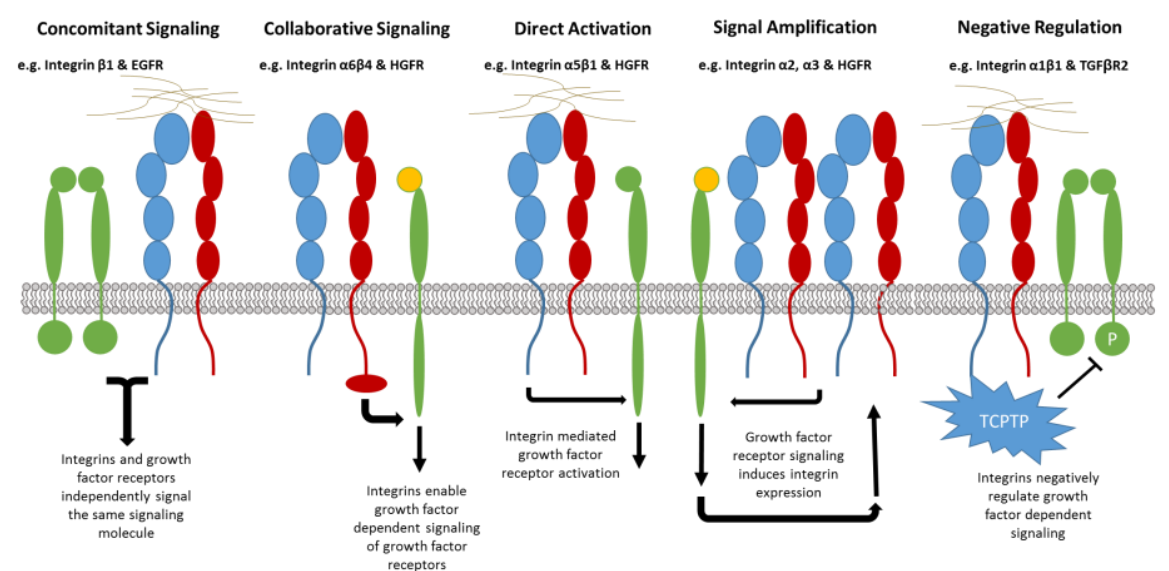

Figure 1. Schematic representation and description of the distinct mechanisms by which integrins and growth factor receptors regulate the activation of signaling pathways within the cell. During concominant signaling, integrins together with growth factor receptors, signal independently to trigger the same signaling molecule. Thereby, integrins gather a platform of signaling proteins, which facilitate growth factor receptors signaling. During direct activation, integrin activate growth factor receptors signaling independent of growth factors. In collaborative signaling, integrin receptors create an permissive environment which enables growth factor receptors to interact with downstream signaling molecules. Growth factor receptor signaling trigger increased integrin expression, which can further induce growth factor receptor signaling. Intergrin interaction with the ECM can cause negative regulation of growth factor receptor signaling through phosphatase activation and recruitment of, for example, T cell protein tyrosine phosphatase (TCPTP). Integrins that are involved in the different signaling 
mechanism are stated as examples. The cooperation between integrins and growth factor recepors has been reviewed in more detail by Ivaska et. al. ${ }^{13}$.

\subsubsection{Integrin and growth factor receptor interactions}

The following are some examples showing interactions between integrins and growth factor receptors in relation to fibrosis and cancer. Growing evidence suggests that the progression of fibrosis is affected by signaling between integrins, growth factor receptors and cytokine or chemokine receptors. Next to cell adhesion, migration, invasion and survival, integrin crosstalk also affect the host response to fibrosis driven diseases ${ }^{8}$.

\section{(i) Interaction with TGF $\beta R$ :}

Transforming growth factor beta 1 (TGF $\beta 1$ ), in its secreted form, is one of the main pro-fibrotic cytokine and regulator of fibrosis in multiple organs ${ }^{3}$. Most of the pro-fibrogenic TGF $\beta 1$ is secreted and bound to the ECM in its latent form ${ }^{3}$. Conversion of latent TGF $\beta 1$ into its active form is an important step that regulates TGF $\beta 1$ activity ${ }^{3}$. $\alpha \mathrm{v}$ integrins are known for their ability to activate latent TGF $\beta 1^{4}$. Evidence for the interaction between TGF $\beta$ and integrins came from the structural analysis of the molecule by Ruoslahti and Pierbacter et al. ${ }^{30}$, which suggested that TGF $\beta 1$ \& TGF $\beta 3$ bind to integrins based on their linear sequence of arginine, glycine and aspartic acid (RGD), which is known to be crucial for the interaction of many integrins with their respective ligands. The integrins $\alpha v \beta 1, \alpha v \beta 3, \alpha v \beta 5, \alpha v \beta 6$ and $\alpha v \beta 8$ were identified to bind to the RGD-sequence of the latency associated peptide (LAP) of TGF $\beta 1$ and TGF $\beta 3$, and are capable to activate latent TGF $\beta{ }^{31-35}$. During pulmonary inflammation and fibrosis, TGF $\beta 1$ activation is regulated by integrin $\alpha v \beta 6^{36}$. Integrin $\alpha v \beta 6$ activates TGF $\beta$ by inducing a conformational change in the integrin $\alpha \mathrm{v} \beta 6$-bound latent TGF $\beta$ complex which then presents active TGF $\beta$ to its receptor on adjacent cells via cell-cell contact ${ }^{31,36}$. Marsh et $a l .{ }^{37}$ showed that integrin $\alpha v \beta 6$ dependent activation of TGF $\beta$ resulted in the differentiation of human fibroblasts into tumor stroma-associated myofibroblasts. Myofibroblasts are capable to activate TGF $\beta 1$ from selfgenerated deposits in the ECM by means of $\alpha v \beta 5$ integrins which transmits the highly contractile forces of these cells to the latent complex of TGF $\beta 1$. Additionally, integrin $\alpha v \beta 8$ has been shown to activate TGF $\beta$ by presenting the latent TGF $\beta$ complex to metalloproteinases that cleave the complex, resulting in the release of free TGF $\beta$ into the extracellular milieu ${ }^{36}$. 
Integrin $\alpha v \beta 3$ is known to induce EMT in mammary epithelial cells by cooperating with TGF $\beta$ via Src-dependent phosphorylation of TGF $\beta$ receptor type $2^{38}$. Integrin $\beta 3$ deficiency in mice was shown to correlate with elevated levels of TGF $\beta$ receptor 1 and 2, reduced levels of Smad 3, sustained nuclear localization of Smad $2 \& 4$ and TGF $\beta 1$-mediated fibroblast migration ${ }^{39}$. These data indicate that integrin $\alpha v \beta 3$ is expressed on platelets, macrophages, endothelial cells and fibroblasts during wound repair and is capable of repressing TGF $\beta 1$-mediated signaling ${ }^{39}$. Increased expression of $\alpha v \beta 5$ in fibroblasts increases their responsiveness to TGF $\beta 1$ by recruiting latent TGF $\beta 1$ on the cell surface and stimulating the interaction between $\alpha v \beta 5$ and the TGF $\beta$ receptor ${ }^{40}$.

\section{(ii) Interaction with VEGFR2:}

During angiogenesis, endothelial cells express integrin $\alpha \mathrm{v} \beta 5$ that interacts with VEGF receptor 2 (VEGFR2) to promote VEGF-induced angiogenesis via the Ras-ERK pathway ${ }^{41,42}$. Additionally, integrin $\alpha v \beta 5$ in cooperation with VEGFR2 causes inflammatory mediators (e.g. tumor necrosis factor) induced resistance of endothelial cells to extrinsic apoptosis, via Src-dependent phosphorylation of Raf Tyr340 and Tyr341 ${ }^{5}$. Mice with genetic knockout of integrin $\beta 3$ showed an abnormal endothelial cell morphology which was associated with increased VEGF signaling ${ }^{43}$. Integrins also play a major role in the control of neo-vascularization in wound healing, where they act as coreceptors for the growth factor-receptors VEGF and angiopoietin, and support the assembly of vascular membranes ${ }^{6}$.

\section{(iii) Interaction with FGFR:}

In endothelial cells, cross-talk between integrin $\alpha v \beta 3$ and fibroblast growth factor receptor (FGFR) was found to induce angiogenesis downstream of FGF binding ${ }^{42}$. Evidences support that FGFR and integrin $\alpha v \beta 3$ cooperate to increase the phosphorylation of Raf Ser338 and Ser 339 through PAK (p21-Activated Protein Kinases) pathway, resulting in Raf-ASK1 complex formation in mitochondria, thereby inhibiting the intrinsic apoptosis pathway ${ }^{8}$. Furthermore, knockdown studies in mice have shown that integrin $\beta 4$ expression correlates with decreased FGF-induced angiogenesis and reduced tumor size via P-ERK and NF-kappaB signaling pathways ${ }^{44}$.

Integrin $\alpha 1 \beta 1$ negatively regulates epidermal growth factor (EGF) receptormediated Rac activation, thereby reducing the production of reactive oxygen species in mesangial cells resulting in attenuation of fibrogenesis in mice ${ }^{45}$. Additionally, other studies have shown that integrin $\alpha 1 \beta 1$ also regulates 
activation of TCPTP, resulting in the inhibition of EGFR and VEGFR2 signaling 46,47 .

\section{(iv) Interaction with other growth factor receptors:}

Integrin $\beta 4$ was found to functionally collaborate with the Met tyrosine kinase receptor for hepatocyte growth factor (HGF) resulting in increased fibroblast transformation and tumorigenic potential ${ }^{48}$. The expression of insulin-like growth factor 2 (IGF-2) has shown to be stimulated by the expression of integrin subunit $\alpha 11 \beta 1$ in stromal fibroblast of non-small-cell lung carcinoma ${ }^{49}$.

In primary cultures of hepatic stellate cells, the liver fibrosis promoting connective tissue growth factor, CTGF (or CCN2), was shown to regulate the expression of integrins on hepatic stellate cells (HSCs) and additionally facilitate HSCs adhesion via binding to integrin $\alpha 5 \beta 1$, which interacts cooperatively with heparin sulfate proteoglycans or fibronectin ${ }^{50}$.

\subsection{Integrins as key targets in wound healing, fibrosis and tumor stroma}

The following section describes the cell type specific expression of integrins (summarized in Figure 2) as well as their functional role in wound healing, tumor stroma, metastasis and kidney, liver and lung fibrosis (Table 2).

\subsubsection{Wound healing}

Wound healing is a common repair process after an injury to an organ. We describe the repair of cutaneous wounds in this section. Wound healing is based on the collective efforts of soluble mediators, blood cells, extracellular matrix and parenchymal cells ${ }^{51}$. This process consists of three timely overlapping stages namely, inflammation, tissue formation, and tissue remodeling ${ }^{51}$. During the innate inflammation, immune cells including neutrophils and granulocytes in the early phase and then macrophages, lymphocytes and mast cells are recruited into the wound. These immune cells release cytokines and chemokines that recruit epithelial cells and fibroblasts to the wound edge ${ }^{6}$. Thereafter, epithelial cells start to stretch into the wound bed, followed by proliferating keratinocytes, which seed more cells into the wound site during re-epithelialization ${ }^{6}$. Parallel to re-epithelialization, granulation tissue formation is initiated which is closely associated with wound angiogenesis ${ }^{6}$. During this process, epithelial cells are recruited into the wound by cytokines and create the granulation tissue together 
with myofibroblasts and pericytes ${ }^{6}$. Myofibroblasts facilitate wound contraction and closure which is followed by tissue remodeling, during which myofibroblasts degrade, remodel and reorganize the ECM ${ }^{6}$. During the complex wound healing process, cells bind to ECM molecules within the wound via their integrin receptors resulting in integrin's functional activation or induced expression. Integrin receptors with a functional role in wound healing are listed in table 2.

Integrin $\beta 1$ as an important integrin because it is a subunit in many different heterodimers. Integrin $\beta 1$ deficiency in mouse fibroblasts, correlates with reduced expression of $\alpha$-smooth muscle actin ( $\alpha$-SMA), CCN2/CTGF and collagen I, and is accompanied by a reduced ability to activate latent TGF $\beta$, thereby inhibiting differentiation of fibroblasts into myofibroblasts, resulting in delayed wound closure and reduced formation of granulation tissue ${ }^{52}$. Integrin $\alpha v \beta 3$ has shown to be inhibitory to the fibroblast infiltration into the wound clot. Mice deficient of integrin $\beta 3$ showed accelerated re-epithelialization, which is associated with enhanced TGF $\beta$ signaling and dermal fibroblast infiltration into wounds ${ }^{39}$. Integrin $\alpha 1 \beta 1$ is expressed on cells of the basement membrane including vascular, visceral, endothelial, smooth muscle cells and pericytes, and on cells of the connective tissue including fibroblasts, chondrocytes, mesenchymal stem cells and circulating white blood cells ${ }^{53}$. The collagens that bind integrin $\alpha 1 \beta 1$ include collagen I, III, IV, XIII, XVI ${ }^{53}$. Modulating the collagen-binding integrin activity could therefore also be an interesting approach to improve the healing of chronic wounds.

Integrin $\alpha 2 \beta 1$ is expressed on keratinocytes, epithelial cells and endothelial cells which are in contact with the basement membrane and on fibroblasts, T-cells, myeloid cells, megakaryocytes and platelets which are in contact with matrices rich in collagen I ${ }^{54}$. Integrin $\alpha 2 \beta 1$ specifically binds to collagen I, III, IV, V, XI, XVI and XXIII ${ }^{54}$. Next to collagens, integrin $\alpha 2 \beta 1$ also binds to the proteoglycans, biglycan, lumican, fibromodulin and decorin, and a proteolytic fragment derived from perlecan called endorepellin ${ }^{54}$. It has been shown that binding of fibroblasts to collagen within 3D-matrices activates p38 $\alpha$ MAP kinase pathway and results in increased integrin $\alpha 2 \beta 1$-dependent collagenase-3 (MMP13) synthesis ${ }^{54,55}$.

Mice deficient in $\alpha 1$ and $\alpha 2$ demonstrated minor changes in their ability to remodel granulation tissue ECM, this is likely because other collagen-binding integrins compensate for most of their function, including MMP expression and 
collagen fibrillogenesis ${ }^{6}$. Integrin $\alpha 3 \beta 1$ is expressed on basal cells of epidermis and other epithelia ${ }^{56}$. Conditional knockout of integrin $\alpha 3 \beta 1$ in the epidermis of mice (keratinocytes) resulted in impaired angiogenesis within wounds, which is correlated with reduced expression of the angiogenesis promoting mitogenregulated protein 3 (MRP3) ${ }^{57}$. These finding suggest a role of $\alpha 3 \beta 1$ in promoting wound angiogenesis through MRP3-mediated crosstalk from epidermal to endothelial cells ${ }^{57}$.

During wound healing, fibronectin (Fn) is activated and assembled into a fibrillary structure Fn matrix that is known to be promoted by integrin $\alpha 5 \beta 1$. Fibroblasts adhesion to the provisional matrix (composed of Fn and fibrin) via integrin $\alpha 5 \beta 1$ in the initial stage of wound healing is enhanced when dermatopontin (a dermal ECM protein) co-localizes with fibrin and fibronectin in the wound clots ${ }^{58}$. Additionally, the interaction of $\alpha 5 \beta 1$ with fibronectin has shown to contribute to T-Cell lymphokine-driven keratinocyte proliferation next to facilitating matrix adhesion and motility ${ }^{59}$. It has been speculated that in fibroblasts, integrin $\alpha 5 \beta 1$ plays an important role in vivo during invasion of connective tissue cells into the wound clot and their migration in the fibrinfibronectin-containing 3D wound environment ${ }^{6}$. Fibroblasts in the granulation tissue have a reduced ability to bind fibronectin via integrin $\alpha 5 \beta 1$ which might allow them to migrate in the early fibronectin-rich granulation tissue matrix ${ }^{60}$. Blocking of integrin $\alpha 5$ with antibodies in vitro in human oral mucosa and dermal fibroblasts were capable of blocking TGF $\beta$-induced expression of $\alpha$ SMA ${ }^{61}$. This finding implies that novel therapeutic approaches targeting integrin $\alpha 5$ could present a strategy to inhibit $\alpha$-SMA positive myofibroblasts which are closely associated with scar formation and various other pathological disorders.

In literature, the increased integrin $\alpha 9 \beta 1$ expression is shown to induce retarded wound re-epithelialization, as shown in $\alpha 9 \beta 1$-deficient mice ${ }^{6}$. Furthermore, integrin $\alpha 9 \beta 1$ controls proliferation of keratinocytes and dermal fibroblast by interacting with elastic microfibril interface-located protein 1 (EMILIN1) ${ }^{62}$. Additionally, blocking of integrin $\alpha 9 \beta 1$ on integrin-positive dermal fibroblasts with a specific antibody inhibited the formation of granulation tissue in cutaneous wound healing, showing that integrin $\alpha 9 \beta 1$ is involved in the formation of granulation tissue by regulating the migration and adhesion of dermal fibroblasts in excisional wounds ${ }^{63}$.

Integrin $\alpha 11 \beta 1$ expression has also been shown to be expressed restrictively to a subset of fibroblasts and mesenchymal stem cells in vivo ${ }^{64}$ and is the main 
collagen receptor on dermal fibroblasts, contributing to collagen remodeling in a TGF $\beta$-dependent manner ${ }^{65}$. Zweers et al. were the first to demonstrate the role for integrin $\alpha 11 \beta 1$ in dermal wound healing, in which $\alpha 11 \beta 1$ is strongly induced in mice after inflicting excisional wounds ${ }^{66}$. Dermal wounds in integrin $\alpha 11 \beta 1$ deficient mice showed a reduction in granulation tissue formation and wound strength 7 days after excisional wound infliction, which is attributed to a defect in myofibroblasts differentiation, indicating $\alpha 11 \beta 1$-dependent collagen remodeling within granulation tissue ${ }^{65}$. Next to its role in collagen remodeling, integrin $\alpha 11 \beta 1$, similar to integrin $\alpha 5 \beta 1$, is involved in myofibroblast differentiation and granulation tissue formation, as a response to injury, and thereby contributes to scar formation. This implies integrin $\alpha 11 \beta 1$ as a potential therapeutic target and it would be of very high interest to investigate the effects of therapeutic blocking of this integrin in the context of myofibroblast differentiation during scar formation and pathological fibrosis in general.

In addition to fibroblasts and epithelial cells, integrins play a key role in immune cells such as neutrophils, monocytes and certain lymphocytes. Integrin $\alpha \mathrm{M} \beta 2$ is a leukocyte receptor which is involved in immune cell recruitment and the activation of inflammatory reposes during wound healing ${ }^{67}$. Integrin $\alpha \mathrm{M} \beta 2$ is capable of engaging various ligands including ECM proteins, counter receptors as intracellular adhesion molecule 1 (ICAM-1) and coagulation and complement products. Ligand binding by integrin $\alpha \mathrm{M} \beta 2$ affects leukocyte adhesion and activation ${ }^{67}$. Knockout of integrin $\alpha \mathrm{M} \beta 2$ in mice has been shown to be correlated with a delay in wound re-epithelialization and granulation tissue formation but did not affect monocyte migration into the wound ${ }^{67}$.

\subsubsection{Fibrosis}

In general, fibrosis is a response to organ injury and progressive fibrosis can lead to major organ failure and ultimately lethality ${ }^{3,68}$. Organ fibrosis is characterized by a complex interplay between inflammatory, epithelial, myofibroblast, and excessive ECM production and deposition ${ }^{3,68}$. The highly dynamic pericellular ECM of the fibrotic tissue exerts profound influences on the behavior of the surrounding cells ${ }^{3}$. Many of the main cell-cell and cell-matrix interactions that regulate fibrosis are mediated by integrins ${ }^{3}$. The expression and function of integrins in kidney, liver and lung fibrosis are described in the following section and is listed in table 2 . 


\subsubsection{Kidney fibrosis}

Integrins $\alpha 1 \beta 1$ and $\alpha 2 \beta 1$, the major collagen binding receptors, and laminin receptors $\alpha 3 \beta 1$ and $\alpha 6 \beta 1$ are highly expressed in the healthy kidney ${ }^{69}$. Integrin $\alpha 1 \beta 1$ binds to collagen IV, and deletion or inhibition of $\alpha 1 \beta 1$ exacerbates glomerulosclerosis suggesting that activation of $\alpha 1 \beta 1$ integrin might be beneficial for renal injury ${ }^{70}$. In contrast to Integrin $\alpha 1 \beta 1$, integrin $\alpha 2 \beta 1$ is a positive regulator of collagen synthesis and reactive oxygen species production. Studies propose that Integrin $\alpha 2 \beta 1$ induces glomerular fibrosis and absence of $\alpha 2 \beta 1$ delays kidney fibrosis and glomerular injury in experimental models for kidney disease ${ }^{71,72}$. Knockdown of the discoidin domain receptor 1 (DDR1) and integrin $\alpha 2 \beta 1$ delays the maturation of the glomerular basement membrane, which causes renal fibrosis in the Col4A3-/- mice, a mouse model of Alport syndrome ${ }^{72}$. Additionally, in Col4A3-/- Alport mice with impaired glomerular basement membrane, maturation loss of integrin $\alpha 2 \beta 1$ delays kidney fibrosis ${ }^{72}$. An additional collagen binding integrin, integrin subunit $\alpha 11$ is specifically localized on myofibroblasts in UUO kidneys in mice and human fibrotic kidneys was found to be crucial for the regulation of the myofibroblast phenotype ${ }^{73}$. Moreover, its expression was significantly induced at an interstitial fibrosis and tubular atrophy score of 3 , when compared to score 0-2 ${ }^{73}$. In Alport mice with a conditional knockdown of integrin $\beta 6$, renal fibrosis was inhibited ${ }^{74}$. Furthermore, knockdown of integrin $\beta 6$ partly or completely protects mice from tubulointerstitial fibrosis induced by kidney obstruction ${ }^{68}$.

The expression of integrin $\alpha v \beta 1, \alpha v \beta 3$ and $\alpha v \beta 5$ was also identified on renal fibroblasts and blockade of $\alpha v \beta 1$ prevented the activation of latent TGF $\beta 1$ through direct binding by fibroblasts ${ }^{75}$.

While in the healthy kidney, integrin $\alpha 8$ is only expressed in mesangial cells and vascular smooth muscle cells, de-novo expression of integrin $\alpha 8$ was found on interstitial fibroblasts and tubular epithelial cells undergoing de-differentiation in tubulointerstitial fibrosis induced by the unilateral ureteral obstruction model ${ }^{76}$. Furthermore, studies in mice with knockdown of integrin $\alpha 8$ revealed that underexpression of $\alpha 8$ did not inhibit tubulointerstitial fibrosis, but increased tubulointerstitium damage compared to wild type mice ${ }^{76}$. Therefore, integrin $\alpha 8$ targeting does not seem to be a useful anti-fibrotic therapeutic target.

Moreover, integrin $\alpha 3$ has shown to induce kidney damage attributed to loss of E-cadherin induced by integrin $\alpha 3$-dependent Src/p- $\beta$-catenin-Y654/p-Smad2mediated up-regulation of integrin-linked kinase ${ }^{77}$. 
In summary, integrin $\alpha 1 \beta 1, \alpha 2 \beta 1, \alpha v \beta 3, \alpha 3$ and $\alpha 8$ have been identified to play a role in kidney fibrosis. Inhibiting integrin $\alpha 2 \beta 1$ and $\alpha \mathrm{v} \beta 3$ in kidney fibrosis seems have high potential as a potential therapy, while induction of integrin $\alpha 1 \beta 1$ and integrin $\alpha 3$, via e.g. an RNAi approach, also seems to have therapeutic potential.

\subsubsection{Liver fibrosis}

In healthy liver, different integrins are expressed at different cell types controlling specific functions to maintain homeostasis. Vascular endothelium expresses many integrins such as $\alpha 1,2,3,4,5$ and 6; bile duct epithelium express integrin $\alpha 2,3,5$ and 6 ; stroma of the connective tissue integrin $\alpha 1$ and 2; hepatocytes integrin $\alpha 1$ and 5; sinusoidal lining cells integrin $\alpha 1,2$, and 5; and mononuclear cells integrin $\alpha 4{ }^{78}$. During liver pathogenesis, the altered expression levels and de novo expression of integrins have been reported in preclinical and clinical studies. Neijjari et al. performed a clinical study including 94 patients with chronic hepatitis $\mathrm{C}$, in which the expression of integrin $\beta 1, \alpha 1, \alpha 5$ and $\alpha 6$ was significantly upregulated and showed correlation with the stage of fibrosis ${ }^{79}$. In a different study, integrin $\beta 6$ was shown to correlate with the stage of fibrosis in the livers of patients with end-stage liver disease, including chronic hepatitis $\mathrm{B} \& \mathrm{C}$, primary biliary cholangitis (PBC) and primary sclerosing cholangitis (PSC) ${ }^{80}$. Mice with integrin $\beta 6$ knockdown or blocking antibody to $\alpha v \beta 6$ significantly decreased acute biliary fibrosis after bile duct ligation ${ }^{68}$. In patients with alcoholic liver disease, integrin $\beta 1$ was significantly overexpressed on $\mathrm{T}$ lymphocytes (and/or hepatocytes) when compared with healthy patients ${ }^{81,82}$. In liver cirrhosis, integrin $\alpha \mathrm{L}, \alpha \mathrm{M}, \alpha \mathrm{X}$ and $\alpha 4$ expression levels on peripheral blood leukocytes was positively correlated with liver failure ${ }^{83}$. During cholestasis, the hepatocytes show de novo expression of integrin $\alpha 3$ and $\alpha 6$, indicating that they undergo a phenotypic switch from hepatocytes to bile duct epithelium ${ }^{78}$. In PBC, integrin $\alpha \mathrm{L} \beta 2$ (lymphocyte function associated antigen 1 (LFA-1)) and $\alpha 4 \beta 1$ (very late antigen 1 (VLA-4)) were found to be expressed on infiltrating lymphocytes, while control livers showed no or weak expression of these integrins ${ }^{84}$.

In addition to these clinical studies, integrin $\alpha v \beta 6$ was found to be upregulated in rotavirus-induced biliary atresia, resulting in liver fibrosis ${ }^{85}$. Rats with a chronic alcohol intoxication showed an induced expression of integrin $\beta 2$ on neutrophils, which increases their migration (most probably mediated by osteopontin via $\alpha 4 \beta 1$ and $\alpha 9 \beta 1$ integrins ${ }^{86}$ ) and Kupffer cells mediated release of chemotactic cytokines and growth factors ${ }^{87}$. Moreover, in PBC, integrin $\alpha \mathrm{L}$ 
(CD11a) was found to be expressed on T lymphocytes but is absent in chronic hepatitis $\mathrm{C}$ or healthy patients, indicating a role of CD4+ integrin $\alpha \mathrm{L}$ expressing lymphocytes in Th-1 predominance and might thereby indirectly promote fibrosis ${ }^{88}$. Mice with an integrin $\beta 2$ knockout showed reduction in hepatic necrosis, decreased number of intrahepatic neutrophils and plasma transaminase activity during acute and chronic cholestatic liver injury ${ }^{89,90}$. In chronic liver inflammation and fibrogenesis, integrin $\beta 1$ activated by vascular adhesion protein-1 and $\mathrm{CX}_{3}$ chemokine receptor 1 has been proposed to be responsible for the recruitment of CD16(+) monocytes into the liver ${ }^{91}$. Activation of HSCs increases the expression of integrin $\alpha 5 \beta 1$, and $\alpha 5 \beta 1 / \mathrm{ECM}$ crosstalk induced collagen production via changes in the cytoskeletal organization, and activation of Src kinases and ERK/JNK signaling molecule families ${ }^{92}$. Expression of integrin $\alpha 8 \beta 1$ is induced in activated HSCs following bile duct ligation or $\mathrm{CCl}_{4}$ induced hepatic injury in rats ${ }^{93}$. Genetic knockdown (Pdgfrb-Cre) of integrin $\alpha \mathrm{v}$ protected mice from CCL4-induced liver fibrosis and was also protective in pulmonary and renal fibrosis ${ }^{94}$. Very recently, Bansal et al. ${ }^{73}$, have identified integrin subunit $\alpha 11$ as a major regulator in the activation of HSCs into myofibroblasts. Knockdown of integrin subunit $\alpha 11$ in HSCs inhibited their differentiation and functionality in response to TGF- $\beta^{73}$. Integrin subunit $\alpha 11$ expression was found to be regulated by the hedgehog pathway and inhibition of hedgehog led to inhibition of HSC induced fibrosis in mice. This work highlights integrin subunit $\alpha 11$ as a highly promising therapeutic target in liver fibrosis ${ }^{73}$.

\subsubsection{Lung fibrosis}

Integrin $\alpha v \beta 6$, a receptor for the ECM proteins fibronectin ${ }^{95}$ and tenascin $\mathrm{C}^{36}$ is minimally expressed in aveolar epithelial tissues but is highly induced upon lung injury, resulting in lung fibrosis ${ }^{96}$. Integrin $\alpha v \beta 6$ has been demonstrated to be overexpressed in the epithelium of lung sclerosis and pulmonary fibrosis ${ }^{68}$. Genetic knockdown of integrin $\beta 6$ in mice was introduced to attenuate bleomycin-induced pulmonary fibrosis and radiation induced pulmonary fibrosis 31,68 . Recently, integrin $\alpha 6 \beta 1$, upregulated in fibrotic lung myfibroblasts, was identified as a mechanosensor for matrix stiffness, ${ }^{97}$. Upon sensing matrix stiffening during pulmonary fibrosis $\alpha 6 \beta 1$ mediates MMP-2 dependent pericellular proteolysis of basement membrane collagen IV, thereby regulating invasion of myofibroblasts ${ }^{97}$. The expression of integrin $\alpha v \beta 8$ is highly expressed in the airways of chronic obstructive pulmonary disease (COPD) patients and correlates with the severity of the obstruction, and COPD fibroblasts show increased pro-fibrogenic differentiation upon $\alpha v \beta 8$ mediated TGF $\beta 1$ 
activation ${ }^{98,99}$. Knockdown of $\alpha v \beta 8$ in murine lung fibroblasts reduced TGF $\beta$ activation in these cells ${ }^{100}$. Deletion of $\alpha \mathrm{v} \beta 8$ in lung fibroblasts resulted in inhibition of airway fibrosis in IL-1 $\beta$ and ovalbumin-induced mouse models ${ }^{100}$. Additionally, the authors demonstrated that IL- $1 \beta$ increased $\alpha v \beta 8$-dependent TGF $\beta$ activation, collagen expression and pro-inflammatory gene expression in human COPD compared to normal human lung fibroblasts ${ }^{100}$. In patients with idiopathic pulmonary fibrosis, integrin $\alpha \mathrm{v} \beta 5$ causes TGF $\beta$-mediated fibrosis and co-localizes with PAR1 (Protease-activated receptors) and the myofibroblast marker $\alpha \mathrm{SMA}^{101}$. This process is inhibited by the blockade of integrin $\alpha v \beta 5$ in mice ${ }^{101}$. Kim et al. showed that in idiopathic pulmonary fibrosis (IPF), alveolar epithelial cells undergo extracellular matrix triggered EMT, thereby turning into differentiating fibroblasts ${ }^{102}$. In an IPF mouse model with a lung specific deletion of integrin $\alpha 3 \beta 1$ reduced accumulation of myofibroblasts, collagen and genes associated with EMT were observed ${ }^{103,104}$. Integrin subunit $\alpha 11$ was in an additional study found to be significantly induced in the fibrotic lungs from patients with IPF were it co-localizes with $\alpha$-SMA-positive myofibroblasts ${ }^{73}$. In addition, the expression of integrin subunit $\alpha 11$ correlated concomitantly with the expression of various fibrotic parameters in the lungs of patients with IPF ${ }^{73}$.

\subsubsection{Tumor stroma}

The tumor stroma consists of non-cancerous cells including cancer-associated fibroblasts (CAFs), tumor-associated macrophages (TAMs), pericytes, endothelial cells, and infiltrating immune cells ${ }^{105}$. More recently, stromal cells have been indentified as drivers of tumorigenesis, promoting tumor growth, angiogenesis, invasion and metastasis ${ }^{105}$. Integrins that were found to be expressed in these cell types and their functional role in the stroma is are discussed in the following section and listed in table 2.

Integrin $\alpha 11 \beta 1$ and $\alpha 5 \beta 1$ have been reported to be the main integrins expressed on fibroblasts within the tumor stroma ${ }^{106,107}$. Integrin $\alpha 11 \beta 1$ was found to be induced in a mechano-sensitive manner and contributes to TGF $\beta$-dependent myofibroblasts differentiation in vitro ${ }^{108}$. A role for integrin $\alpha 11 \beta 1$ in tumorigenesis was first observed in lung adenocarcinoma in which $\alpha 11 \beta 1$ was identified as a tumor biomarker ${ }^{109}$. Specifically, integrin $\alpha 11 \beta 1$ is found to be overexpressed in the tumor stromal tissue of lung adenocarcinoma, where it induces IGF2 expression and tumorigenicity ${ }^{49}$. Furthermore, Integrin $\alpha 11 \beta 1$ is overexpressed in the tumor stroma of head and neck squamous cell carcinoma and its expression positively correlates and co-localizes with the expression of 
aSMA, a myofibroblast marker with prognostic value in head and neck squamous cell carcinoma ${ }^{106}$. Additionally, the gene encoding for integrin $\alpha 11 \beta 1$ (ITGA11), next to six other genes, was found to promote invasion, in a spheroid based model for the invasion of breast cancer cells ${ }^{110}$. Moreover, integrin $\alpha 11 \beta 1$ plays a role in the paracrine signaling between fibroblasts and cancer cells within tumor stroma. A study using 3D-heterospheroids composed of mouse embryonic fibroblasts (MEFs) and A549 lung carcinoma cells showed that CXCL5 expression in tumor cells was inversely related to integrin $\alpha 11 \beta 1$ in MEFs, indicating that integrin $\alpha 11 \beta 1$ increases the autocrine secretion of CXCL5 by lung carcinoma cells ${ }^{111}$. Franco-Barraza et al. have very recently introduced that desmoplastic traits, prognostic of neoplastic recurrence, dependent on integrin $\alpha 5 \beta 1$, expressed on myofibroblasts in pancreatic cancer, are maintained by matrix-regulated integrin $\alpha v \beta 55^{107}$. In this work, the author's identified a CAF phenotype, with high expression of active integrin $\alpha 5 \beta 1$. Finally they propose a novel prognostic tool, in which they use stromal localization and levels of active Smad $2 / 3$ and integrin $\alpha 5 \beta 1$ to distinguish patient-protective from patientdentrimental desmoplasia, to foretell pancreatic cancer recurrence

In addition to fibroblasts, integrins on macrophages also play a key role in tumor stroma. A recent study has implicated integrins in regulating the ability of TAMs to promote tumor progression. For example, in a melanoma model, an osteopontin-rich matrix activates TAMs through ligation of integrin $\alpha 9 \beta 1$, stimulating the migration of endothelial and cancer cells via prostaglandin E2 production ${ }^{112}$. Similarly, the ECM protein periostin, secreted by glioblastoma stem cells, promote TAM recruitment to tumors via activation of $\alpha \mathrm{v} \beta 33^{113}$.

These studies indicate that integrin $\alpha 11 \beta 1, \alpha 5 \beta 1, \alpha 9 \beta 1$ and $\alpha v \beta 3$ play a crucial role in the tumor stroma by controlling the phenotype and behavior of key stromal cells. The integrins $\alpha 11 \beta 1, \alpha 9 \beta 1$ and $\alpha v \beta 3$ show potential as therapeutic targets. In the context of drug delivery integrin $\alpha 11 \beta 1$ presents as a target with high potential, since the expression of this integrin is restricted to tumor stroma or other fibrotic disease but is generally not expressed in other tissues of the adult body. The design of novel integrin targeting ligands is therefore a highly interesting approach for drug delivery to CAFs.

\subsubsection{Metastasis}

Metastasis, which is defined as the spreading of cells from the primary tumor site to other organs is responsible for more than $90 \%$ of cancer-related lethality 
${ }^{114}$. Integrins, which are regulators of cell attachment to the ECM as well as cell migration and therefore have a crucial part in the regulation of metastasis ${ }^{115}$.

Integrin $\alpha 2$ is widely expressed which makes it difficult to determine its function during tumorigenesis. High expression of integrin $\alpha 2$ in breast and prostate cancer correlates with a favorable prognosis ${ }^{116}$. Additionally, MMTV-neu mice lacking integrin $\alpha 2$ expression had increased tumor cell intravasation indicating that integrin $\alpha 2$ has metastasis suppressing properties ${ }^{116}$. Additionally, integrin $\alpha 2 \beta 1$ expression causes a decrease in lymph node metastasis in human papilloma virus-induced squamous cell carcinoma in mice ${ }^{117}$. In contradiction, elevated levels of integrin $\alpha 2 \beta 1$ accelerated experimental metastasis in melanoma, gastric and colon cancer ${ }^{118-120}$. Moreover, integrin $\alpha 2 \beta 1$ rich xenograft tumors, in mice have shown to promote metastasis to the bone ${ }^{121}$.

The role of integrin $\alpha 2$ in tumorigenesis and metastasis is not yet clarified and appears to be dependent on expression levels and the tumor type ${ }^{64}$. Zeltz and Gullberg ${ }^{64}$ hypothesized that high expression of integrin $\alpha 2$ in welldifferentiated tumors might prevent metastasis, while tumors at other stages with low integrin $\alpha 2$ expression might support dedifferentiation and metastasis by directing metastasizing cells to collagen rich tissues, such as bone . In summary, integrin $\alpha 2 \beta 1$ could be further exploited as a biomarker. Since studies in integrin $\alpha 2$ knockout mice show no significant side effects integrin $\alpha 2 \beta 1$ additionally presents a potential therapeutic target to attenuate metastasis, but this strategy seems only applicable for not-well-differentiated tumors with low collagen expression.

In a clinical study including lung cancer patients with brain metastases (BM), high expression of integrin $\alpha v \beta 5$ on vascular structures and tumor stroma (in $\mathrm{BM})$ was found to be associated with high hypoxia inducible factor $1 \alpha(\mathrm{HIF} 1 \alpha)$ indices, while $\alpha \mathrm{v} \beta 3$ was expressed on vascular structures and tumor cells (in $\mathrm{BM}$ ) and was correlated with low Ki-67 indices ${ }^{122}$. Although it would be of high interest to see what effects new therapeutic agents against $\alpha v \beta 5$ might have on the expression of HIF1 $\alpha$ in BM, it could be argued that $\alpha \mathrm{v}$ integrins are not a feasible therapeutic target, because blocking of these integrins could be associated with severe side effects, due to their wide expression in the human body. Still the expression of $\alpha \mathrm{v}$ integrins are of pathological and clinical relevance in lung cancer patients with brain metastasis and it might be interesting to explore them as biomarkers. 
In colorectal cancer, CD98, integrin $\beta 1, \beta 3$ and FAK was overexpressed and correlated with cancer progression and metastasis in the liver ${ }^{123}$. Direct contact between the tumor stroma and tumor cells is required for these markers to be over-expressed in metastasis of the liver ${ }^{123}$.

Integrins seem to play a crucial role in the regulation of metastasis within certain tumors. Especially integrin $\alpha 2 \beta 1$ has, dependent on tumor stage and type, potential as a therapeutic target in metastasis, while integrin $\alpha v \beta 3, \alpha v \beta 5, \beta 1$ and $\beta 3$ should be validated for their use as biomarkers. Unfortunately, none of the integrins discussed show a very specific expression in pathological tissues, but are widely expressed, which makes these integrins poor targets for drug targeting.

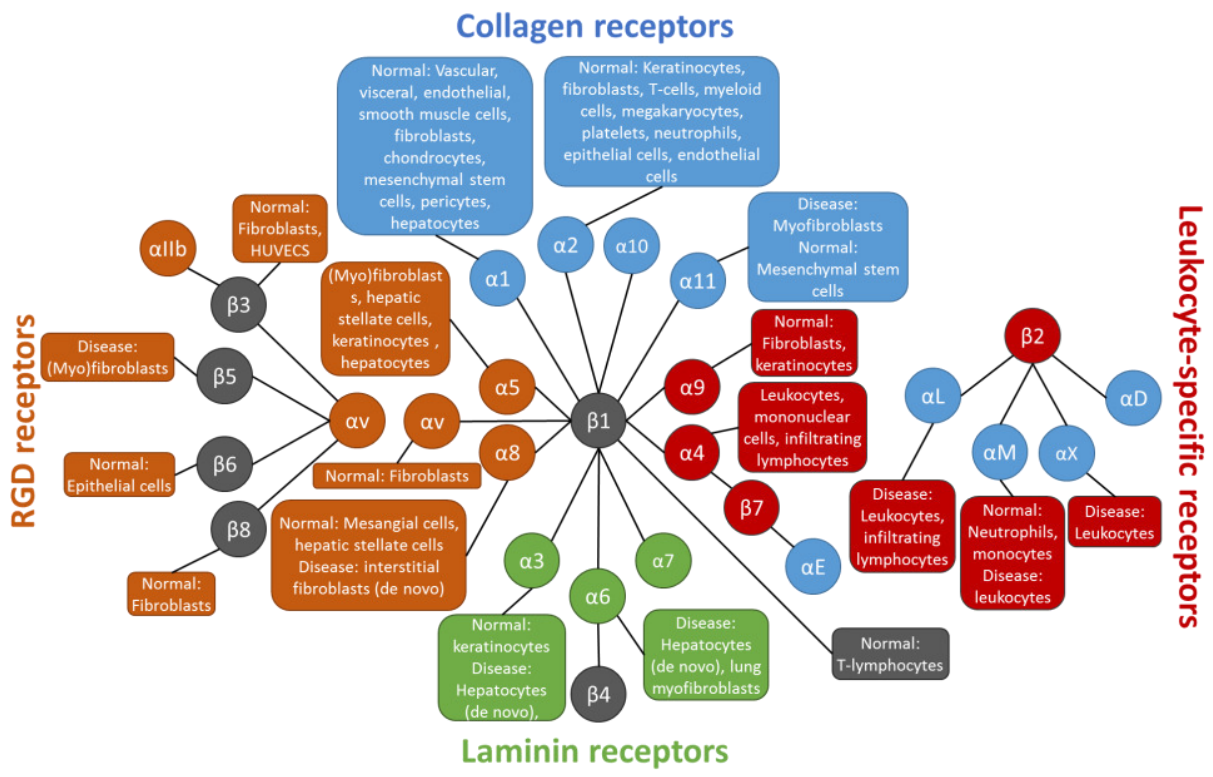

Figure 2. Schematic representation of the 24 different integrin receptor pairs, including their ligand specificity and cellular expression in fibrosis, tumor stroma and wound healing. The integrins cellular expression has been subdivided into nonpathogenic cell, named "normal", and pathogenic cells, named "disease". 
Table 2. Integrins and their function in wound healing, tumor stroma, metastasis, kidney, liver and lung fibrosis.

\begin{tabular}{|c|c|c|c|c|}
\hline Integrin & $\begin{array}{l}\text { Expressional } \\
\text { Modification }\end{array}$ & Function & $\begin{array}{l}\text { Cell / Tissue } \\
\text { Type/Disease }\end{array}$ & Ref. \\
\hline \multicolumn{5}{|c|}{ Wound Healing } \\
\hline$\beta 3$ & $\begin{array}{l}\text { Genetic } \\
\text { knockdown }\end{array}$ & $\begin{array}{l}\text { Accelerated re-epithelialization, } \\
\text { enhanced TGF } \beta \text { signaling, } \\
\text { dermal fibroblast infiltration }\end{array}$ & $\begin{array}{l}\text { Fibroblasts, } \\
\text { Epithelial cells }\end{array}$ & 39 \\
\hline$\beta 1$ & $\begin{array}{l}\text { Fibroblast } \\
\text { specific } \\
\text { knockout }\end{array}$ & $\begin{array}{l}\text { Delayed cutaneous wound } \\
\text { closure and reduced granulation } \\
\text { tissue formation, including } \\
\text { reduced production of new ECM } \\
\text { and reduced } \alpha \text { SMA expression }\end{array}$ & Fibroblasts & 52 \\
\hline$\alpha 3 \beta 1$ & $\begin{array}{l}\text { Epidermal } \\
\text { knockout }\end{array}$ & Impaired angiogenesis & $\begin{array}{l}\text { Keratinocytes, } \\
\text { Endothelial cells }\end{array}$ & 57 \\
\hline$\alpha 5 \beta 1$ & Overexpression & $\begin{array}{l}\text { Interaction of overexpressed } \\
\text { integrin } \alpha 5 \beta 1 \text { leads to } \mathrm{T} \text { Cell } \\
\text { driven keratinocyte proliferation }\end{array}$ & Keratinocytes & 59 \\
\hline$\alpha 5 \beta 1$ & None & $\begin{array}{l}\text { Granulation tissue fibroblasts } \\
\text { have a reduced ability to bind } \\
\text { fibronectin via } \alpha 5 \beta 1 \text {, increasing } \\
\text { their migration ability }\end{array}$ & Fibroblasts & 60 \\
\hline$\alpha 9$ & None & $\begin{array}{l}\text { Regulates migration and } \\
\text { adhesion of dermal fibroblasts } \\
\text { during granulation tissue } \\
\text { formation in excisional wounds. }\end{array}$ & $\begin{array}{l}\text { Dermal } \\
\text { Fibroblasts }\end{array}$ & 63 \\
\hline$\alpha \mathrm{M} \beta 2$ & $\begin{array}{l}\text { Genetic } \\
\text { knockdown }\end{array}$ & $\begin{array}{l}\text { Delayed wound re- } \\
\text { epithelialization }\end{array}$ & $\begin{array}{l}\text { Neutrophils, } \\
\text { monocytes }\end{array}$ & 67 \\
\hline$\alpha v \beta 6$ & Overexpression & 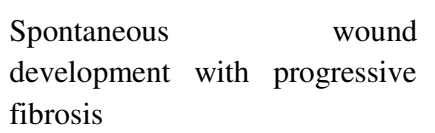 & Epithelial cells & 124 \\
\hline$\alpha v \beta 6$ & $\begin{array}{l}\text { Genetic } \\
\text { knockdown }\end{array}$ & Delayed wound healing & Epithelial cells & 125 \\
\hline$\alpha v \beta 5$ & None & $\begin{array}{l}\text { Expressed in deep human and } \\
\text { porcine wound during early re- } \\
\text { epithelialization }\end{array}$ & Epidermis & 126 \\
\hline$\alpha v \beta 5$ & None & $\begin{array}{l}\text { Induced in keratinocytes during } \\
\text { late mucosal and dermal wound } \\
\text { healing }\end{array}$ & Keratinocytes & 127 \\
\hline
\end{tabular}




\begin{tabular}{|c|c|c|c|c|}
\hline Integrin & $\begin{array}{l}\text { Expressional } \\
\text { Modification } \\
\end{array}$ & Function & $\begin{array}{l}\text { Cell I Tissue } \\
\text { Type/Disease }\end{array}$ & Ref. \\
\hline$\alpha 2 \beta 1$ & $\begin{array}{l}\text { Antibody } \\
\text { blocking }\end{array}$ & $\begin{array}{l}\text { Induces MMP-1 expression and } \\
\text { collagen matrix denaturation in } \\
\text { wounds }\end{array}$ & Keratinocytes & 128 \\
\hline$\alpha 5 \beta 1$ & Overexpression & $\begin{array}{l}\text { Affects fibronectin integration } \\
\text { and restricts keratinocyte } \\
\text { Migration }\end{array}$ & Keratinocytes & 129,130 \\
\hline$\alpha 11 \beta 1$ & $\begin{array}{l}\text { Genetic } \\
\text { knockdown }\end{array}$ & $\begin{array}{l}\text { Reduced granulation tissue } \\
\text { formation and impaired wound } \\
\text { contraction }\end{array}$ & Fibroblasts & 131 \\
\hline$\alpha 3 \beta 1$ & None & $\begin{array}{l}\text { Determines the migrational } \\
\text { directionality of keratinocytes }\end{array}$ & Keratinocytes & 132 \\
\hline$\beta 1$ & $\begin{array}{l}\beta 1 \quad \text { deficient } \\
\text { keratinocytes }\end{array}$ & 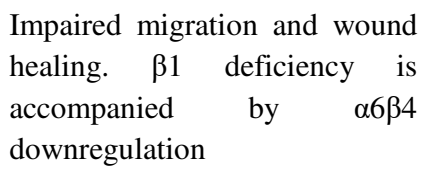 & Keratinocytes & 133 \\
\hline$\alpha 3 \beta 1$ & $\begin{array}{l}\text { Genetic } \\
\text { knockdown }\end{array}$ & $\begin{array}{l}\text { Inhibits directional migration } \\
\text { and re-epithelialization }\end{array}$ & Keratinocytes & 134 \\
\hline \multicolumn{5}{|c|}{ Kidney Fibrosis } \\
\hline$\beta 6$ & $\begin{array}{l}\text { Genetic } \\
\text { knockdown }\end{array}$ & $\begin{array}{l}\text { Prevents tubulointerstitial } \\
\text { fibrosis }\end{array}$ & Kidney & 68 \\
\hline$\alpha 3$ & $\begin{array}{l}\text { Genetic } \\
\text { knockdown }\end{array}$ & $\begin{array}{l}\text { Reduced neovascularization; } \\
\text { delayed kidney fibrosis and } \\
\text { neovascularization }\end{array}$ & Kidney & $71,72,135$ \\
\hline$\alpha 2 \beta 1$ & $\begin{array}{l}\text { Genetic } \\
\text { knockdown }\end{array}$ & $\begin{array}{l}\text { Knockdown delays the } \\
\text { maturation of the glomerular } \\
\text { basement membrane and kidney } \\
\text { fibrosis in Alport mice }\end{array}$ & Kidney & 72 \\
\hline$\alpha 11 \beta 1$ & None & $\begin{array}{l}\text { Crucial for the regulation of the } \\
\text { myofibroblast phenotype }\end{array}$ & $\begin{array}{l}\text { Myofibroblasts in } \\
\text { UUO kidenys in } \\
\text { mice and human } \\
\text { fibrotoc kidneys }\end{array}$ & 73 \\
\hline$\beta 6$ & $\begin{array}{l}\text { Genetic } \\
\text { knockdown }\end{array}$ & $\begin{array}{l}\text { Knockdown inhibits renal } \\
\text { fibrosis in Alport mice }\end{array}$ & Kidney & 74 \\
\hline$\alpha v \beta 1$ & None & $\begin{array}{l}\text { blockade of } \alpha v \beta 1 \text { prevents the } \\
\text { activation of latent TGF } \beta 1 \\
\text { through direct binding by } \\
\text { fibroblasts }\end{array}$ & $\begin{array}{l}\text { Fibroblasts in the } \\
\text { Kidney }\end{array}$ & 75 \\
\hline
\end{tabular}




\begin{tabular}{|c|c|c|c|c|}
\hline Integrin & $\begin{array}{l}\text { Expressional } \\
\text { Modification } \\
\end{array}$ & Function & $\begin{array}{l}\text { Cell I Tissue } \\
\text { Type/Disease }\end{array}$ & Ref. \\
\hline$\alpha 8$ & $\begin{array}{l}\text { Genetic } \\
\text { knockdown }\end{array}$ & $\begin{array}{l}\text { De novo expression in interstitial } \\
\text { fibroblasts and tubular } \\
\text { endothelial cells in } \\
\text { tubulointerstitium fibrosis. } \\
\text { Knockdown did not inhibit } \\
\text { tubulointerstitium fibrosis, but } \\
\text { increased tubulointerstitium } \\
\text { damage compared to wild type } \\
\text { mice }\end{array}$ & $\begin{array}{l}\text { Fibroblasts and } \\
\text { endothelial cells } \\
\text { in } \\
\text { tubulointerstitium } \\
\text { fibrosis }\end{array}$ & 76 \\
\hline$\alpha 3$ & None & $\begin{array}{l}\text { Mediates kidney fibrosis via } \\
\text { integrin-linked kinase through } \\
\text { mediated loss of E-cadherin }\end{array}$ & Kidney fibrosis & 77 \\
\hline \multicolumn{5}{|c|}{ Liver Fibrosis } \\
\hline$\beta 6$ & $\begin{array}{l}\text { Genetic } \\
\text { knockdown }\end{array}$ & Prevents acute biliary fibrosis & Liver & 68 \\
\hline$\alpha 3, \alpha 6$ & None & $\begin{array}{l}\text { Expression of these integrins } \\
\text { indicate a switch of hepatocytes } \\
\text { into bile duct epithelial cells }\end{array}$ & $\begin{array}{l}\text { Hepatocytes in } \\
\text { cholestasis }\end{array}$ & 78 \\
\hline $\begin{array}{l}\beta 1, \quad \alpha 1 \\
\alpha 5, \alpha 6\end{array}$ & None & $\begin{array}{l}\text { Integrin expression positively } \\
\text { correlates with the stage of } \\
\text { fibrosis }\end{array}$ & Liver & 79 \\
\hline$\beta 1$ & None & $\begin{array}{l}\text { Integrin expression level } \\
\text { positively correlates with the } \\
\text { stage of fibrosis. }\end{array}$ & $\begin{array}{l}\text { Chronic hepatitis } \\
\text { C \& B, PBC, PSC }\end{array}$ & 80 \\
\hline$\beta 1$ & None & $\begin{array}{l}\begin{array}{l}\text { Overexpressed } \\
\text { lymphocytes }\end{array} \\
\text { lym }\end{array} \quad \begin{array}{l}\mathrm{T} \\
\end{array}$ & $\begin{array}{l}\text { Alcoholic liver } \\
\text { disease }\end{array}$ & 81 \\
\hline $\begin{array}{l}\alpha \mathrm{L}, \quad \alpha \mathrm{M} \\
\alpha \mathrm{X}, \alpha 4\end{array}$ & None & $\begin{array}{l}\text { Expression levels on peripheral } \\
\text { blood leukocytes positively } \\
\text { correlated with liver failure }\end{array}$ & $\begin{array}{l}\text { Leukocytes in } \\
\text { liver cirrhosis }\end{array}$ & 83 \\
\hline $\begin{array}{l}\alpha \mathrm{L} \beta 2, \\
\alpha 4 \beta 1\end{array}$ & None & $\begin{array}{l}\text { Integrin expression correlates } \\
\text { with infiltrating lymphocytes }\end{array}$ & $\begin{array}{l}\text { Lymphocytes in } \\
\text { PBC }\end{array}$ & 84 \\
\hline$\alpha v \beta 6$ & None & Upregulated & Biliary atresia & 85 \\
\hline$\beta 2$ & None & $\begin{array}{l}\text { Induced expression of integrin } \\
\beta 2 \text { on neutrophils increases their } \\
\text { migration and Kupffer cell } \\
\text { release of chemotactic cytokines } \\
\text { and growth factors }\end{array}$ & $\begin{array}{l}\text { Chronic alcohol } \\
\text { intoxication of } \\
\text { the liver }\end{array}$ & 87 \\
\hline
\end{tabular}




\begin{tabular}{|c|c|c|c|c|}
\hline Integrin & $\begin{array}{l}\text { Expressional } \\
\text { Modification }\end{array}$ & Function & $\begin{array}{l}\text { Cell / Tissue } \\
\text { Type/Disease }\end{array}$ & Ref. \\
\hline$\alpha \mathrm{L}$ & None & Indirectly promotes fibrosis & $\begin{array}{l}\text { Lymphocytes in } \\
\text { PBC }\end{array}$ & 88 \\
\hline$\beta 6$ & $\begin{array}{l}\text { Genetic } \\
\text { knockdown }\end{array}$ & $\begin{array}{l}\text { Knockdown inhibits neutrophil } \\
\text { infiltration and plasma } \\
\text { transaminase activity as well as } \\
\text { hepatic necrosis }\end{array}$ & & 89 \\
\hline$\beta 6$ & $\begin{array}{l}\text { Genetic } \\
\text { knockdown }\end{array}$ & $\begin{array}{l}\text { Knockdown inhibits neutrophil } \\
\text { infiltration and plasma } \\
\text { transaminase activity as well as } \\
\text { hepatic necrosis }\end{array}$ & $\begin{array}{l}\text { Acute cholestatic } \\
\text { hepatitis }\end{array}$ & 90 \\
\hline$\beta 1$ & None & $\begin{array}{l}\text { Responsible for the recruitment } \\
\text { of CD16(+) monocytes into the } \\
\text { liver }\end{array}$ & $\begin{array}{l}\text { Chronic liver } \\
\text { inflammation and } \\
\text { fibrogenesis }\end{array}$ & 91 \\
\hline$\alpha 5 \beta 1$ & None & $\begin{array}{l}\text { Increases collagen production } \\
\text { via integrin } \alpha 5 \beta 1 / \mathrm{ECM} \text { induced } \\
\text { changes in the cytoskeletal } \\
\text { organization and activation of } \\
\text { Src kinases and ERK/JNK }\end{array}$ & $\begin{array}{l}\text { Hepatic stellate } \\
\text { cells in liver } \\
\text { fibrosis }\end{array}$ & 92 \\
\hline$\alpha v$ & $\begin{array}{l}\text { Genetic } \\
\text { knockdown }\end{array}$ & $\begin{array}{l}\text { Protected mice from CCL4- } \\
\text { induced liver fibrosis and was } \\
\text { also protective in pulmonary and } \\
\text { renal fibrosis }\end{array}$ & Myofibroblasts & 94 \\
\hline$\alpha 11 \beta 1$ & $\begin{array}{l}\text { Genetic } \\
\text { knockdown }\end{array}$ & $\begin{array}{l}\text { Major regulator in the activation } \\
\text { of HSCs into myofibroblasts }\end{array}$ & $\begin{array}{l}\text { Myofibroblasts / } \\
\text { Liver Fibrosis in } \\
\text { mice }\end{array}$ & 73 \\
\hline \multicolumn{5}{|c|}{ Lung Fibrosis } \\
\hline$\alpha v \beta 6$ & $\begin{array}{l}\text { Genetic } \\
\text { knockdown }\end{array}$ & $\begin{array}{l}\text { Regulates pulmonary fibrosis } \\
\text { and inflammation by activating } \\
\text { TGF } \beta\end{array}$ & $\begin{array}{l}\text { Epithelial cells of } \\
\text { lung sclerosis and } \\
\text { pulmonary } \\
\text { fibrosis }\end{array}$ & 31 \\
\hline$\alpha v \beta 8$ & $\begin{array}{l}\text { Genetic } \\
\text { knockdown }\end{array}$ & $\begin{array}{l}\text { Increased pro-fibrotic } \\
\text { differentiation of lung } \\
\text { fibroblasts by regulating TGF } \beta \\
\text { activation. Increases the } \\
\text { expression of collagen and pro- } \\
\text { fibrotic genes. }\end{array}$ & COPD fibroblasts & $98-100$ \\
\hline$\alpha v \beta 5$ & None & Mediates TGF $\beta$ induced fibrosis. & $\begin{array}{l}\text { Fibroblasts of } \\
\text { pulmonary } \\
\text { fibrosis }\end{array}$ & 101 \\
\hline
\end{tabular}




\begin{tabular}{|c|c|c|c|c|}
\hline Integrin & $\begin{array}{l}\text { Expressional } \\
\text { Modification }\end{array}$ & Function & $\begin{array}{l}\text { Cell / Tissue } \\
\text { Type/Disease }\end{array}$ & Ref. \\
\hline$\alpha 3 \beta 1$ & $\begin{array}{l}\text { Genetic } \\
\text { knockdown }\end{array}$ & $\begin{array}{l}\text { Knockdown correlates with } \\
\text { reduced accumulation } \\
\text { myofibroblasts, collagen and } \\
\text { EMT-associated genes }\end{array}$ & $\begin{array}{l}\text { Pulmonary } \\
\text { fibrosis }\end{array}$ & 103,104 \\
\hline$\alpha 11 \beta 1$ & none & $\begin{array}{l}\text { Expression correlated } \\
\text { concomitantly with the } \\
\text { expression of various fibrotic } \\
\text { parameters in the lungs of } \\
\text { patients with IPF }\end{array}$ & $\begin{array}{l}\text { Idiopathic } \\
\text { pulmonary } \\
\text { fibrosis }\end{array}$ & 73 \\
\hline \multicolumn{5}{|c|}{ Tumor Stroma } \\
\hline$\alpha 11 \beta 1$ & $\begin{array}{l}\text { Genetic } \\
\text { knockout }\end{array}$ & $\begin{array}{l}\text { Expression positively correlates } \\
\text { with prognosis. Induces IGF2 } \\
\text { expression and tumorigenicity. } \\
\text { Induces CXCL5 secretion by } \\
\text { lung carcinoma cells. }\end{array}$ & $\begin{array}{l}\text { Fibroblasts in } \\
\text { Lung } \\
\text { adenocarcinoma }\end{array}$ & $49,109,111$ \\
\hline$\alpha 11 \beta 1$ & None & $\begin{array}{l}\text { Overexpressed in the tumor } \\
\text { stroma. Expression positively } \\
\text { correlates and co-localizes with } \\
\text { the expression of } \alpha \mathrm{SMA}\end{array}$ & $\begin{array}{l}\text { Head and neck } \\
\text { squamous cell } \\
\text { carcinoma }\end{array}$ & 106 \\
\hline$\alpha 5 \beta 1$ & None & $\begin{array}{l}\text { Desmoplastic traits prognostic } \\
\text { of neoplastic recurrence of } \\
\text { integrin } \alpha 5 \beta 1 \text { are maintained by } \\
\text { matrix regulated integrin } \alpha v \beta 5\end{array}$ & $\begin{array}{l}\text { Cancer- } \\
\text { associated } \\
\text { fibroblasts in } \\
\text { pancreatic cancer }\end{array}$ & 107 \\
\hline$\alpha 11 \beta 1$ & None & Promotes invasion & $\begin{array}{l}\text { Invasive breast } \\
\text { cancer cells }\end{array}$ & 110 \\
\hline$\alpha 9 \beta 1$ & None & $\begin{array}{l}\text { Osteopontin-rich matrix } \\
\text { activates TAMs through ligation } \\
\text { of integrin } \alpha 9 \beta 1 \text {, stimulating the } \\
\text { migration of endothelial and } \\
\text { cancer cells via prostaglandin E2 } \\
\text { production }\end{array}$ & Melanoma model & 112 \\
\hline$\alpha v \beta 3$ & None & $\begin{array}{l}\text { Periostin, secreted by } \\
\text { glioblastoma stem cells, promote } \\
\text { TAM recruitment to tumors via } \\
\text { integrin } \alpha v \beta 3 \text { signaling. }\end{array}$ & $\begin{array}{l}\text { Glioblastoma } \\
\text { xenografts }\end{array}$ & 113 \\
\hline \multicolumn{5}{|c|}{ Metastasis } \\
\hline$\alpha 2 \beta 1$ & $\begin{array}{l}\text { Genetic } \\
\text { knockdown }\end{array}$ & $\begin{array}{l}\text { Expression is associated with } \\
\text { favourable prognosis and } \\
\text { reduced tumor cell intravasation }\end{array}$ & $\begin{array}{l}\text { Breast Cancer, } \\
\text { squamous cell } \\
\text { carcinoma }\end{array}$ & 116,117 \\
\hline
\end{tabular}




\begin{tabular}{|l|l|l|l|l|}
\hline Integrin & $\begin{array}{l}\text { Expressional } \\
\text { Modification }\end{array}$ & Function & $\begin{array}{l}\text { Cell / Tissue } \\
\text { Type/Disease }\end{array}$ & Ref. \\
\hline$\alpha 2 \beta 1$ & None & $\begin{array}{l}\text { Accelerated levels increase } \\
\text { experimental metastasis }\end{array}$ & $\begin{array}{l}\text { metastasis in } \\
\text { melanoma, } \\
\text { gastric and colon } \\
\text { cancer }\end{array}$ & $118-120$ \\
\hline$\alpha v \beta 5$ & None & $\begin{array}{l}\text { Expressed in vasclular structures } \\
\text { and tumor stroma and associated } \\
\text { with high hypoxia inducible } \\
\text { factor 1 } \alpha \text { indices }\end{array}$ & $\begin{array}{l}\text { Brain metastasis } \\
\text { of lung cancer }\end{array}$ & 122 \\
\hline$\alpha v \beta 3$ & None & $\begin{array}{l}\text { Expressed on vascular structures } \\
\text { and associated with low Ki-67 } \\
\text { indices }\end{array}$ & $\begin{array}{l}\text { Brain metastasis } \\
\text { of lung cancer }\end{array}$ & 122 \\
\hline$\beta 1, \beta 3$ & None & $\begin{array}{l}\text { Overexpressed and correlate } \\
\text { with cancer progression and } \\
\text { metastasis in the liver. }\end{array}$ & $\begin{array}{l}\text { Liver metastasis } \\
\text { of colorectal } \\
\text { cancer }\end{array}$ & 123 \\
\hline
\end{tabular}

\subsection{Therapies based on integrins inhibition}

Various different integrins have been identified to play a role in fibrosis and their knockdown or blocking has been shown to dampen disease progression. An example of a clinically approved integrin inhibitor is the integrin $\alpha 4 \beta 7$ inhibitor vedolizumab, selectively inhibiting lymphocyte trafficking, which is applied as a treatment in Crohn's disease ${ }^{136}$. Therefore integrin-specific inhibitors have a huge potential as anti-fibrotic therapeutics. An overview of therapies (preclinical and clinical) based on integrin inhibition are described in the following section and are summarized in Table 3.

\subsection{1 av-Family integrins}

The majority of integrin targeting drugs tested in clinical trials inhibit $\alpha \mathrm{v}$ integrins. In the context of this review, it is important to realize that $\alpha \mathrm{v}$ integrins are generally expressed in the blood vessels and various other endothelial tissues and in the case of cancer, these drugs may target tumor cells, next to angiogenic vessels of the tumor microenvironment. Although integrins are abundantly present on tumor cells, in this review we focus on tumor stromal cells and we therefore only mention integrin $\alpha \mathrm{v}$ inhibiting therapies, which clearly demonstrated effects on these cells. 
An antibody so-called vitaxin against $\alpha \mathrm{v} \beta 3$, which was later evolved into etaracizumab was tested in phase I and II clinical trials with low toxicity. Therapeutic efficacy but no immunogenicity was observed after treatment with etaracizumab in metastatic melanoma and other solid tumors. However, further development of etaracizumab was terminated based on the results of a randomized clinical trial in which its efficacy was compared to standard chemotherapy showing no meaningful improvement ${ }^{137-140}$.

Another antibody against $\alpha v \beta 3$ and $\alpha v \beta 5$, CTNO 95, has been tested in phase I clinical trials in advanced solid tumors showing anti-tumor activity and no toxicity ${ }^{141}$. Hereupon, CTNO 95 together with docetaxel and prednisolone was evaluated in a multicenter phase II clinical trial for safety and efficacy in patients with castration-resistant prostate cancer in which CTNO 95 caused a shorter progression free survival without showing additional toxicity compared to placebo treatment ${ }^{142}$. Later CNTO 95 was tested in combination with bevacizumab in a phase I biomarker study in patients with advanced solid tumors, could be administered safely and resulted in changes of the plasma levels of soluble endoglin, soluble E-cadherin, and soluble E-selectin as well as PlGF and VEGF-D, all proteins which interact with the ECM ${ }^{143}$. The selective $\alpha v \beta 3$ and $\alpha v \beta 5$ blocker cilengitide, based on the cyclic RGD peptide was successfully tested in phase I and II clinical trials for lung cancer, prostate cancer and glioblastoma but failed to enhance the survival benefit in patients when cilengitide was given in addition to the standard of care therapy ${ }^{144-147}$. Using a small molecule inhibitor for integrin $\alpha v \beta 1$, Reed et al. ${ }^{148}$ found that $\alpha v \beta 1$ directly binds to the latency-associated peptide of TGF $\beta 1$, thereby mediating TGF $\beta$ activation. Administration of this small molecule inhibitor showed therapeutic efficacy by attenuating bleomycin-induced pulmonary fibrosis and carbon tetrachloride-induced liver fibrosis but has not been evaluated in clinical trials 148 .

Antibody mediated blocking of the TGF $\beta$ activating integrin $\alpha \mathrm{v} \beta 6$ has shown therapeutic activity in a wide range of pre-clinical fibrosis models. These models include models for lung fibrosis ${ }^{149,150}$, liver fibrosis ${ }^{151,152}$ and renal fibrosis ${ }^{74,153}$. A humanized monoclonal antibody, STX-100 (BG00011), against av $\beta 6$ is currently being tested in phase 2 clinical trials in patients with idiopathic pulmonary fibrosis ${ }^{154}$. Genetic knockdown of $\beta 6$ and functional antibody blocking of $\alpha v \beta 6$ in renal fibrosis attenuates the accumulation of activated fibroblasts and interstitial collagen matrix deposition ${ }^{74}$. Treatment of scleroderma fibroblasts with antibodies against integrin $\alpha v \beta 3$ and $\alpha v \beta 5$ reduced 
the expression of procollagen type $\mathrm{I}^{33,34,40,155,156}$. Antibody mediated blocking of the integrins $\alpha v \beta 3$ and $\alpha v \beta 5$ inhibits myofibroblast differentiation in oral and dermal fibroblasts in vitro, while the inhibition of differentiation of kidney fibroblasts was only achieved with antibody blocking of $\alpha v \beta 33^{157}$. A monoclonal antibody against $\alpha v \beta 3$ blocked fibroblast growth factor (FGF), tumor necrosis factor-alpha and human melanoma fragments induced angiogenesis in human wound granulation tissue ${ }^{158}$. A small molecule inhibitor of $\alpha v \beta 6$ (EMD527040) inhibited bile duct proliferation and peribiliary collagen deposition, decreased the expression of pro-fibrotic and induced fibrolytic genes ${ }^{159}$. In Mdr2(Abcb4)(I-) mice with spontaneous biliary liver fibrosis, a single dose of a selective $\alpha v \beta 6$ inhibitor significantly induced profibrolytic MMP-8 \& -9 , and showed downregulation of the fibrosis markers procollagen $\alpha 1$, TGF $\beta 2$ and MMP- ${ }^{80}$.

The integrins $\alpha v \beta 3$ and $\alpha v \beta 6$ targeting peptide ACDCRGDCFC has been conjugated to the pro-apoptotic antimicrobial synthetic peptide (KLAKLAK) $)_{2}$ and has shown selective toxicity to angiogenic endothelial cells, by disrupting their mitochondrial membranes, and showed anti-cancer activity in mice ${ }^{160}$.

Hepatic stellate cells, precursors of liver myofibroblasts, are one of the major sources of ECM production in liver fibrosis, which makes them a target for antifibrotic therapeutics. Zhou et al. found that inhibition of integrin $\alpha v \beta 3$ with neutralizing antibodies, echistatin or small inhibitory RNA to silence the $\alpha \mathrm{v}$ subunit expression, decreased proliferation of hepatic stellate cells ${ }^{161}$. More recently, the $\alpha v \beta 3$ inhibitor Cilengitide was used to treat liver fibrosis in rat, induced by bile duct ligation (BDL) or thioacetamide (TAA) injections, and resulted in a significant decrease of liver fibrosis and collagen deposition, but increased experimental liver fibrosis $(\sim 30 \%){ }^{162}$. By blocking both ICAM-1 and integrin $\alpha \mathrm{v}$ on hepatic stellate cells, phagocytosis of fibrosis promoting lymphocytes, a process mediated through members of the Rho family (Cdc42 or Rac-1) and leading to the activation of hepatic stellate cells was completely prevented ${ }^{163}$.

Genetic knockdown and blocking of integrin $\alpha v \beta 6$ with antibodies prevented radiation-induced pulmonary fibrosis ${ }^{150}$ and hepatic fibrosis induced by biliary obstruction in mice ${ }^{151}$. The small molecule inhibitor C 8 binding to $\alpha \mathrm{v} \beta 1$ in picomolar concentrations, significantly inhibited liver and lung fibrosis in mice, reducing collagen deposition by approximately $50 \%{ }^{148}$. In a renal unilateral obstruction model, administration of C8 inhibited collagen deposition and effectively attenuated renal failure ${ }^{75}$. Another small molecule inhibitor blocking 
$\alpha \mathrm{v}, \mathrm{CWHM}$ 12, attenuated CCl4-induced liver fibrosis, bleomycin-induced lung fibrosis as well as cerulein-induced pancreatic fibrosis in mice ${ }^{94,164}$. Although, most therapeutic approaches inhibiting integrins are directed against $\alpha \mathrm{v}$ there are only a handful of compounds which have made it to clinical trails and those have failed to show improved therapeutic efficacy, which might be related to offtarget binding of these inhibitor due to the wide expression of integrin subunit $\alpha v$.

\subsection{2 $\alpha 5$-Family integrins}

An additional target for anti-cancer therapy is integrin $\alpha 5 \beta 1$, known to be expressed on CAFs, angiogenic vessels and tumor cells. A humanized monoclonal antibody, specifically binding to $\alpha 5 \beta 1$, volociximab, showed absence of severe toxicities in patients with solid tumors and resulted in one minor response and disease stabilization in another case in a phase I clinical trial 165. A phase II study with volociximab was performed in patients with relapsed malignant melanoma showing insufficient effects to proceed to stage 2 of the study ${ }^{166}$. The subsequent phase II study evaluating volociximab in refractory metastatic clear cell renal cancer resulted in stable disease in $87 \%$ of the patients ${ }^{167}$. This study is continued in a follow-up in which higher dose levels are being evaluated patients ${ }^{167}$.

In metastatic pancreatic cancer, volociximab was studied in combination with gemcitabine in which 5\% had confirmed partial response and 50\% of the patients showed a stable disease ${ }^{168}$. In a phase II study in platinum resistant advanced epithelial ovarian or primary peritoneal cancer, volociximab showed insufficient clinical activity ${ }^{169}$. Another study in recurrent ovarian or primary peritoneal cancer, volociximab was used in combination with pegylated liposomal doxorubicin, showing no statistically significant difference when compared to pegylated doxorubicin alone ${ }^{170}$. In a phase I dose escalation study of volociximab in combination with carboplatin and paclitaxel in patients with advanced non-small cell lung carcinoma showed promising clinical efficacy, but the development of this therapy has not been developed further ${ }^{171}$. Another integrin $\alpha 5 \beta 1$ monoclonal antibody developed by Pfizer, PF- 4605412 has also been evaluated in phase I clinical trials and has been discontinued due to the acute infusion-related reactions ${ }^{172}$.

An integrin $\alpha 5 \beta 1$ binding RGD peptide inhibited the progression of CCl4induced liver fibrosis and collagen deposition in the liver ${ }^{173}$. Additionally, RGD inhibited the expression of collagen 1 and tissue inhibitor of MMP-1 and 
increased MMP-1 expression of human hepatic stellate cell derived cells in vitro 173.

Another $\alpha 5 \beta 1$ targeting peptide derived from the synergy region of fibronectin, binding to $\alpha 5 \beta 1$ and $\alpha v \beta 3$, ATN-161 (Ac-PHSCN-NH2) caused a dosedependent decrease in tumor volume and inhibited metastasis in a metastatic mouse breast cancer model ${ }^{174}$. In a phase I clinical trial in patients with solid tumors, ATN-161 caused no dose limiting toxicities ${ }^{175}$. Unfortunately, no objective responses to ATN-161 treatment was found, but prolonged stable disease was observed in patients with renal cancer ${ }^{175}$.

\subsection{3 $\alpha 2$-Family integrins}

A small molecule inhibitor of integrin $\alpha 2 \beta 1$ (E7820) was investigated in phase I clinical trials for the treatment of metastatic colon cancer and is currently tested in phase II clinical trials in combination with certuximab ${ }^{176,177}$

\subsection{4 $\beta 1$-Family integrins}

Since integrin $\beta 1$ is a partner receptor for many $\alpha$ receptor units, it is present ubiquitously. In bevacizumab-resistant glioblastomas (BRG) integrin $\beta 1$ mediates interactions between the tumor and its microenvironment ${ }^{178}$. These interactions include tumor cell binding to ECM-ligands like fibronectin, collaten IV and laminin, and VEGF-dependent as well as independent vascularization ${ }^{178}$. Treatment of BRG mouse xenografts with the integrin $\beta 1$ specific antibody, OS2966, allowed a reduction in the dose of bevacizumab and delivery of OS2966 over a period of 28 days showed increased apoptosis of tumor cells and a decrease in tumor cell invasiveness and mesenchymal morphology of tumor cells 178 .

A 41 amino acid peptide, purified from the venom of the Viperia lebetina obtusa viper is an effective inhibitor of integrin $\alpha 1 \beta 1$ and inhibited angiogenesis and tumor growth in vivo in the chicken chorioallantoic membrane assay and the Lewis lung syngeinic mouse model ${ }^{179}$.

\subsection{5 $\alpha \mathrm{L}, \alpha \mathrm{M}$ and $\beta 2$-Family integrins}

$\alpha \mathrm{L}$ and $\alpha \mathrm{M}$-family integrins antibody mediated blocking of integrin $\alpha \mathrm{L} \beta 2$ in mice that underwent bile duct ligation decreased the activity of alanine aminotransferase and aspartate aminotransferase levels in serum. Additionally the adhesion of leukocytes in bile duct ligation induced post-sinuoidal venules 
was reduced ${ }^{180}$. Integrin $\alpha \mathrm{M}$ and CD44 blockage with antibody reduced the localization of monocytes to hepatic foci ${ }^{181}$. Additionally, treatment of rats with the neutropenic monoclonal antibody $1 \mathrm{~F} 123$ against integrin $\beta 2$, capable of forming heterodimer with integrin $\alpha \mathrm{M}, \alpha \mathrm{L}, \alpha \mathrm{X}$ and $\alpha \mathrm{D}$, attenuated alcohol initiated hepatic injury ${ }^{87}$.

Table 3. Therapeutics for integrin inhibition in development.

\begin{tabular}{|c|c|c|c|c|c|}
\hline $\begin{array}{l}\text { Integrin } \\
\text { Target }\end{array}$ & $\begin{array}{l}\text { Compound } \\
\text { Name }\end{array}$ & Stage (year) & Cellular Target & Disease Target & Ref. \\
\hline \multicolumn{6}{|c|}{$\alpha v$-Family Integrins } \\
\hline$\alpha v \beta 3$ & Vitaxin & $\begin{array}{l}\text { Phase I } \\
(2000)\end{array}$ & $\begin{array}{l}\text { Endothelial } \\
\text { Cells }\end{array}$ & $\begin{array}{l}\text { Breat, Lung and } \\
\text { Colon Cancer }\end{array}$ & 137 \\
\hline$\alpha v \beta 3$ & Etaracizumab & $\begin{array}{l}\text { Phase I } \\
(2005,2008)\end{array}$ & $\begin{array}{l}\text { Endothelial } \\
\text { Cells }\end{array}$ & $\begin{array}{l}\text { Several solid } \\
\text { tumors }\end{array}$ & $\begin{array}{l}138,13 \\
9\end{array}$ \\
\hline$\alpha v \beta 3$ & Etaracizumab & $\begin{array}{l}\text { Phase II } \\
(2010)\end{array}$ & $\begin{array}{l}\text { Endothelial } \\
\text { Cells }\end{array}$ & $\begin{array}{l}\text { Metastatic } \\
\text { melanoma }\end{array}$ & 140 \\
\hline$\alpha \mathrm{v}$ & CTNO 95 & $\begin{array}{l}\text { Phase } \quad \text { I } \\
(2007)\end{array}$ & $\begin{array}{l}\text { Endothelial and } \\
\text { Tumor Cells }\end{array}$ & $\begin{array}{l}\text { Several solid } \\
\text { tumors }\end{array}$ & 141 \\
\hline$\alpha \mathrm{v}$ & CTNO 95 & $\begin{array}{l}\text { Phase II } \\
(2013)\end{array}$ & $\begin{array}{l}\text { Endothelial and } \\
\text { Tumor Cells }\end{array}$ & $\begin{array}{l}\text { Castration- } \\
\text { resistant prostate } \\
\text { cancer }\end{array}$ & 142 \\
\hline$\alpha v$ & CTNO 95 & $\begin{array}{l}\text { Phase I } \\
(2015)\end{array}$ & $\begin{array}{l}\text { Endothelial and } \\
\text { Tumor Cells }\end{array}$ & $\begin{array}{l}\text { Several solid } \\
\text { tumors }\end{array}$ & 143 \\
\hline $\begin{array}{l}\alpha v \beta 3 \\
\alpha v \beta 5\end{array}$ & Cilengitide & $\begin{array}{l}\text { Phase II } \\
(2006)\end{array}$ & $\begin{array}{l}\text { Endothelial and } \\
\text { Tumor Cells }\end{array}$ & Prostate Cancer & 144 \\
\hline $\begin{array}{l}\alpha v \beta 3, \\
\alpha v \beta 5, \\
\alpha v \beta 1\end{array}$ & Cilengitide & 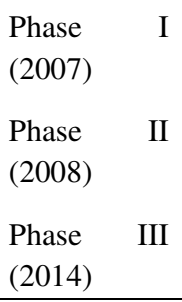 & $\begin{array}{l}\text { Endothelial and } \\
\text { Tumor Cells }\end{array}$ & $\begin{array}{l}\text { Malignant } \\
\text { Glioma, } \\
\text { Glioblastoma }\end{array}$ & $\begin{array}{l}145,14 \\
6,182\end{array}$ \\
\hline$\alpha v \beta 1$ & $\mathrm{C} 8$ & Pre-clinical & $\begin{array}{l}\text { Cancer- } \\
\text { associated } \\
\text { fibroblasts }\end{array}$ & $\begin{array}{l}\text { Liver fibrosis, } \\
\text { Lung fibrosis, } \\
\text { Kideny fibrosis }\end{array}$ & 75,148 \\
\hline
\end{tabular}




\begin{tabular}{|c|c|c|c|c|c|}
\hline $\begin{array}{l}\text { Integrin } \\
\text { Target }\end{array}$ & $\begin{array}{l}\text { Compound } \\
\text { Name }\end{array}$ & Stage (year) & Cellular Target & Disease Target & Ref. \\
\hline$\alpha v \beta 1$ & c8 & Pre-clinical & $\begin{array}{l}\text { Activated } \\
\text { fibroblasts }\end{array}$ & $\begin{array}{l}\text { Bleomycin- } \\
\text { induced } \\
\text { pulmonary } \\
\text { fibrosis, Carbon } \\
\text { tetrachloride- } \\
\text { induced liver } \\
\text { fibrosis }\end{array}$ & 148 \\
\hline$\alpha v$ & CWHM 12 & Pre-clinical & & $\begin{array}{l}\text { CCL4-induced } \\
\text { liver fibrosis, } \\
\text { bleomycin- } \\
\text { induced lung } \\
\text { fibrosis, cerulein- } \\
\text { induced pancreatic } \\
\text { fibrosis }\end{array}$ & 94,164 \\
\hline$\alpha v \beta 6$ & $\begin{array}{l}\text { Anti- } \alpha v \beta 6- \\
m A b\end{array}$ & Pre-clinical & $\begin{array}{l}\text { Acinar cells, } \\
\text { Pancreatic } \\
\text { Stellate Cells }\end{array}$ & $\begin{array}{ll}\text { Lung } & \text { Fibrosis, } \\
\text { Biliary } & \text { Fibrosis, } \\
\text { Liver } & \text { Fibrosis, } \\
\text { Renal } & \text { Fibrosis, } \\
\text { Kidney } & \text { Fibrosis }\end{array}$ & $\begin{array}{l}74,150 \\
-153\end{array}$ \\
\hline$\alpha v \beta 5$ & P1F6 & Pre-clinical & $\begin{array}{l}\text { Kidney } \\
\text { fibroblasts, } \\
\text { Oral fibroblasts, } \\
\text { Dermal } \\
\text { fibroblasts }\end{array}$ & / & 157 \\
\hline$\alpha v \beta 3$ & LM609 & Pre-clinical & $\begin{array}{l}\text { Oral fibroblasts, } \\
\text { Dermal } \\
\text { fibroblasts }\end{array}$ & I & 157 \\
\hline$\alpha v \beta 6$ & STX-100 & $\begin{array}{l}\text { Phase II } \\
(2017)\end{array}$ & I & $\begin{array}{l}\text { Pulmunary } \\
\text { Fibrosis }\end{array}$ & 154 \\
\hline$\alpha v \beta 3$ & $\begin{array}{l}\text { Anti- } \alpha v \beta 3- \\
m A b\end{array}$ & Pre-clinical & $\begin{array}{l}\text { Endothelial } \\
\text { Cells }\end{array}$ & $\begin{array}{l}\text { Human Wound } \\
\text { Tissue }\end{array}$ & 158 \\
\hline$\alpha v \beta 6$ & EMD527040 & Pre-clinical & $\begin{array}{l}\text { Bile duct } \\
\text { epithelial cells, } \\
\text { Mdr2(Abcb4)(- } \\
\text { /-) mice with } \\
\text { spontaneous } \\
\text { biliary fibrosis }\end{array}$ & Liver Fibrosis & 80,159 \\
\hline
\end{tabular}


Chapter 5

\begin{tabular}{|c|c|c|c|c|c|}
\hline $\begin{array}{l}\text { Integrin } \\
\text { Target }\end{array}$ & $\begin{array}{l}\text { Compound } \\
\text { Name } \\
\end{array}$ & Stage (year) & Cellular Target & Disease Target & Ref. \\
\hline $\begin{array}{l}\alpha v \beta 3 \\
\alpha v \beta 6\end{array}$ & $\begin{array}{l}\text { ACDCRGDC } \\
\text { FC- } \\
(\text { KLAKLAK })_{2}\end{array}$ & Pre-clinical & Endothelial cells & I & 160 \\
\hline$\alpha v \beta 3$ & $\begin{array}{l}\text { Echistatin, } \alpha v \\
\text { RNAi, anti } \beta 3\end{array}$ & Pre-clinical & $\begin{array}{l}\text { Hepatic stellate } \\
\text { cells }\end{array}$ & Liver Fibrosis & 161 \\
\hline $\begin{array}{l}\alpha v \beta 3 \\
\alpha v \beta 5\end{array}$ & Cilengitide & Pre-clinical & $\begin{array}{l}\text { Hepatic stellate } \\
\text { cells }\end{array}$ & $\begin{array}{l}\text { Experimental liver } \\
\text { Fibrosis }\end{array}$ & 162 \\
\hline$\alpha \mathrm{v}$ & $\begin{array}{l}\text { Anti-Integrin } \\
\text { alpha V }\end{array}$ & Pre-clinical & $\begin{array}{l}\text { Hepatic Stellate } \\
\text { Cells }\end{array}$ & Liver Fibrosis & 163 \\
\hline \multicolumn{6}{|c|}{ 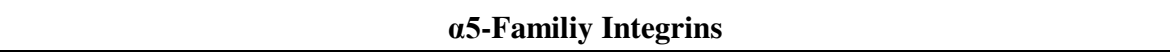 } \\
\hline$\alpha 5 \beta 1$ & Volociximab & $\begin{array}{l}\text { Phase II } \\
\text { (discontinue } \\
\text { d, 2006 - } \\
2011 \text { ) }\end{array}$ & $\begin{array}{l}\text { Endothelial } \\
\text { Cells }\end{array}$ & $\begin{array}{l}\text { Ovarian cancer, } \\
\text { peritoneal cancer, } \\
\text { pancreatic cancer, } \\
\text { renal cancer }\end{array}$ & $\begin{array}{l}166- \\
170\end{array}$ \\
\hline$\alpha 5 \beta 1$ & Volociximab & $\begin{array}{l}\text { Phase I } \\
\text { (discontinue } \\
\text { d, 2008, } \\
2013)\end{array}$ & $\begin{array}{l}\text { Endothelial } \\
\text { Cells }\end{array}$ & $\begin{array}{l}\text { Advanced Solid } \\
\text { Malignancies, } \\
\text { Non-small-cell } \\
\text { lung cancer }\end{array}$ & $\begin{array}{l}165,17 \\
1\end{array}$ \\
\hline$\alpha 5 \beta 1$ & RGD & Pre-clinical & $\begin{array}{l}\text { Hepatic stellate } \\
\text { cells }\end{array}$ & Liver cirrhosis & 173 \\
\hline$\alpha 5 \beta 1$ & $\begin{array}{l}\text { PF-4605412 } \\
\text { (Mab) }\end{array}$ & $\begin{array}{l}\text { Phase I } \\
\text { (discontinue } \\
d, 2013 \text { ) }\end{array}$ & $\begin{array}{l}\text { Endothelial } \\
\text { Cells }\end{array}$ & $\begin{array}{l}\text { Solid } \\
\text { Malignancies }\end{array}$ & 172 \\
\hline $\begin{array}{l}\alpha v \beta 3, \\
\alpha 5 \beta 1\end{array}$ & ATN-161 & Pre-clinical & $\begin{array}{l}\text { Endothelial } \\
\text { Cells }\end{array}$ & Breast cancer & 174 \\
\hline $\begin{array}{l}\alpha v \beta 3, \\
\alpha 5 \beta 1\end{array}$ & ATN-161 & $\begin{array}{ll}\text { Phase } & I \\
(2006) & \\
\end{array}$ & $\begin{array}{l}\text { Endothelial } \\
\text { Cells }\end{array}$ & Solid tumors & 175 \\
\hline \multicolumn{6}{|c|}{$\alpha 2$-Family Integrins } \\
\hline$\alpha 2 \beta 1$ & E7820 & $\begin{array}{ll}\text { Phase } & \text { I } \\
(2011) & \\
\end{array}$ & $\begin{array}{l}\text { Endothelial } \\
\text { Cells }\end{array}$ & $\begin{array}{l}\text { Advanced solid } \\
\text { tumors }\end{array}$ & 176 \\
\hline$\beta 1$ & OS2966 & Pre-clinical & $\begin{array}{l}\text { Endothelial } \\
\text { Cells }\end{array}$ & Glioblastoma & 178 \\
\hline$\alpha 1 \beta 1$ & Obtustatin & Pre-clinical & $\begin{array}{l}\text { Endothelial } \\
\text { Cells }\end{array}$ & Lung cancer & 179 \\
\hline & & & & & \\
\hline
\end{tabular}




\begin{tabular}{|c|c|c|c|c|c|}
\hline \multicolumn{6}{|c|}{$\alpha \mathrm{L}, \alpha \mathrm{M}$ and $\beta 2$-Family Integrins } \\
\hline$\alpha \mathrm{L} \beta 2$ & $\begin{array}{l}\text { anti-LFA-1 } \\
\text { antibody }\end{array}$ & Pre-clinical & Leukocytes & Liver Fibrosis & 180 \\
\hline$\alpha \mathrm{M}$ & $\begin{array}{l}\text { Anti-CD11b } \\
\text { antibody }\end{array}$ & Pre-clinical & Monocytes & Liver Infection & 181 \\
\hline$\beta 2$ & $1 \mathrm{~F} 12$ & Pre-clinical & $\begin{array}{l}\text { Neutrophils in } \\
\text { the liver }\end{array}$ & $\begin{array}{l}\text { Experimental } \\
\text { alcoholic hepatitis }\end{array}$ & 87 \\
\hline
\end{tabular}

\subsection{Drug targeting strategies based on integrin ligands}

Extensive work has been done in the design of new ligands for integrin receptor targeting that can be utilized for integrin facilitated drug delivery or imaging. A large portion of these ligands are targeting RGDs and have been extensively reviewed in a recent article by Arosio et al. ${ }^{183}$. Therefore, RGD based targeting approaches have been excluded from this review, unless it has been shown that they have facilitated specific targeting to components related to fibrosis, wound healing or the tumor stroma.

Ruoslahti et al. have developed two peptides binding to the av binding motifs Arg-Gly-Asp (RGD) and Asn-Gly-Arg (NGR), and coupled these to the anticancer drug doxorubicin. Targeting the tumor vasculature of human breast cancer xenografts in mice, resulted in enhanced efficacy of doxorubicin ${ }^{184}$. Targeting doxorubicin to endothelial cells by linking a bicyclic CDCRGDCFC (RGD-4C) peptide selectively binding to integrin $\alpha v \beta 3$ and $\alpha v \beta 5$ and a D-AlaPhe-Lys tripeptide selectively binding to tumor-associated protease plasmin to the drug, resulted in plasmin-dependent cytotoxicity of endothelial cells ${ }^{185}$. The cyclic RGD compound containing the conformational constrained homoSer-Pro dipeptide unit with a fluorescent probe for $\alpha \mathrm{v} \beta 3$-imaging has shown to possess the ability to bind endothelial cells in vitro ${ }^{186}$. A new $\alpha v \beta 3$-specific tumor vessel binding cyclic peptide containing the iso-DGR motif was conjugated to albumin and coupled to tumor necrosis factor- $\alpha$ (TNF- $\alpha$ ) carrying gold nanoparticles ${ }^{187}$. This nanodrugs showed inhibited tumor growth in WEHI fibrosarcoma bearing mice ${ }^{187}$.

The integrin $\alpha 5 \beta 1$ antagonistic peptide ATN-161 in combination with the chemotherapeutic agent 5-fluorouracil significantly reduced liver metastasis, tumor microvessels as well as increased tumor cell apoptosis and decreased tumor cell proliferation, resulting in an increased overall survival in mice ${ }^{188}$. 
Later, ATN-161 was investigated in phase II trials in cancer ${ }^{189}$. The PHSCNK peptide targeting $\alpha 5 \beta 1$ was applied to modify Dox-loaded liposomes to enable targeting of the tumor vasculature and showed enhanced cytotoxicity to endothelial and breast cancer cells, which was attributed to integrin mediated endocytosis ${ }^{190}$. Mardilovich et al. have designed a integrin $\alpha 5 \beta 1$ specific peptide PR_b, mimicking the cell binding site of native fibronectin ${ }^{191}$. Later this peptide has been applied to target siRNA and encapsulated in polymerosomes, lipoplexes, polyplexes and stealth liposomes to integrin $\alpha 5 \beta 1^{192-194}$.

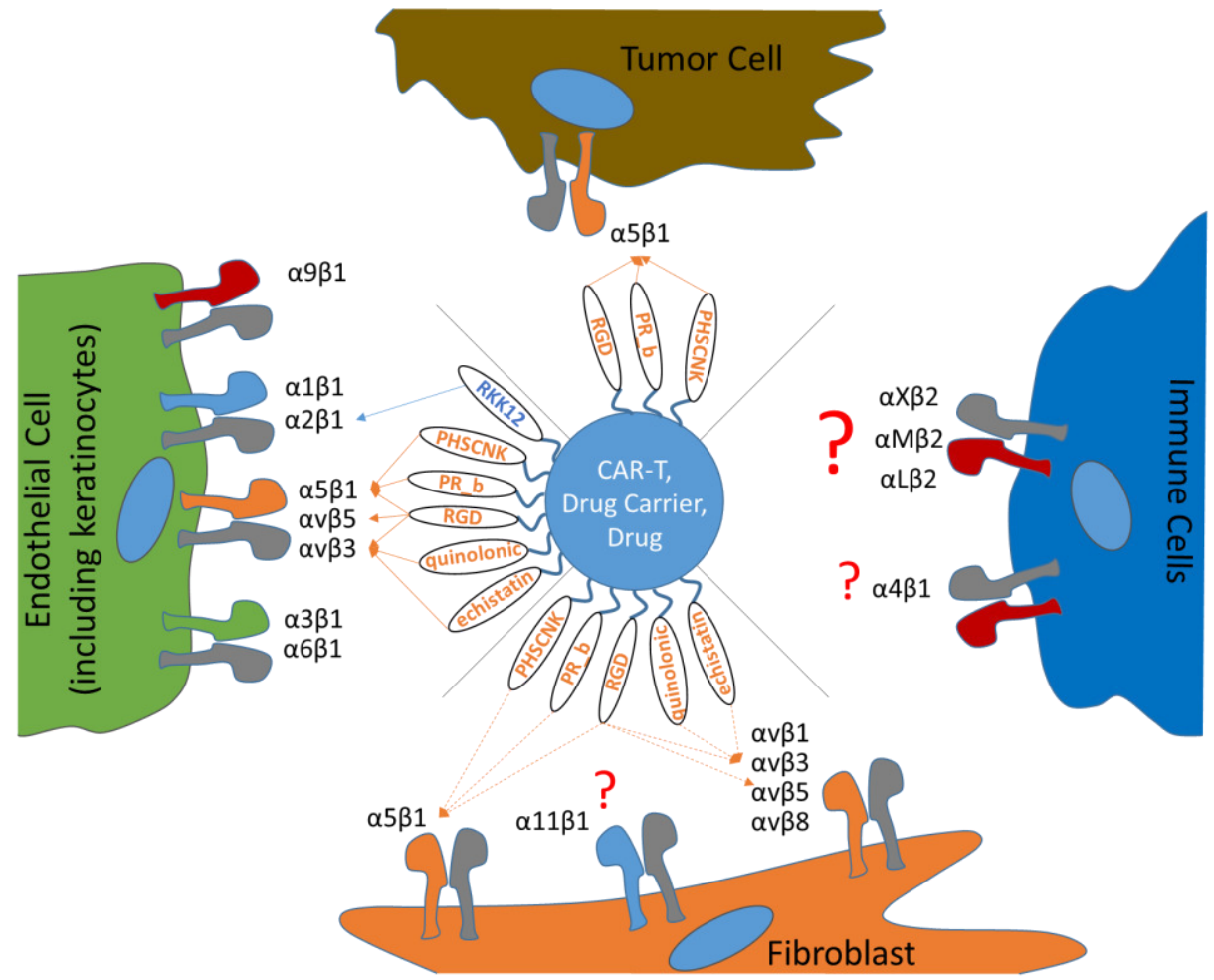

Figure 3. Existing integrin drug targeting ligands, applied for the modification of drug molecules, drug carriers and CAR-T cells (CAR-T), in the context of wound healing, fibrosis or tumor stroma and their respective integrin targets. Dashed lines show potential integrin targets for existing lingands that have not yet been used for targeting the respective cell line. In addition to already targeted integrins, integrins that have not yet been targeted but play a role in the pathology of wound healing, fibrosis or tumor stroma are presented.

Peptidomimetic integrin ligands selectively binding to either $\alpha 5 \beta 1$ or $\alpha v \beta 3$ in fibroblasts functionalized with 1-((1,3-dicarboxy)propyl)-4,7-(carboxymethyl)- 
1,4,7-triazacyclononane (NODAGA) successfully targeted $\alpha 5 \beta 1$ or $\alpha v \beta 3$ expressing tumors in mice. Additionally, both peptidomimetics inhibited tumor growth in syngeneic subcutaneous WEHI-164 fibrosarcomas in mice by inhibiting $\alpha 5 \beta 1$ or $\alpha \mathrm{v} \beta 3$, respectively ${ }^{195,196}$.

Modification of cisplatin with platinum (IV) complexes conjugated to the

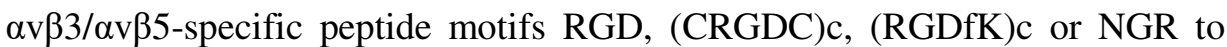
target tumor endothelial cells, showed anti-proliferative effects on human endothelial cells in vitro ${ }^{197}$. Pan et al., have modified PEGylated perfluorooctylbromide (PFOB) nanoparticles (NPs) carrying the anti-angiogenic fumagillin with a quinolonic $\alpha v \beta 3$-integrin ligand conjugated to the PEG chain of these NPs. This drug delivery system decreased angiogenesis in a Matrigel plug assay for angiogenesis in mice ${ }^{198}$. Fu et al., have modified chimeric antigen receptor (CAR) $\mathrm{T}$ cell with echistatin, having a high binding affinity to integrin $\alpha v \beta 3$. The echistatin expressing $T$ cells were capable to efficiently lyse HUVECS, resulting in the extensive bleeding of the tumor tissue in vivo without damaging blood vessels in normal tissues and facilitated specific penetration of liposomes into the tumor site ${ }^{199}$.

\subsection{Conclusions and future directions}

Despite a lot of work being done to gain insight in the biological role of integrin subtypes in wound healing, fibrosis and tumor stroma, there is is still a need to better understand their significance and implications. Most studies have been carried out by the means of integrin subunit knockout in mice or using integrinspecific inhibitory antibodies. A few studies have revealed integrins with a role in fibrosis, which resulted in some potential anti-fibrotic therapeutics. However, most of them failed in early or late clinical trials. It is worth mentioning that integrin $\alpha \mathrm{v}$ and $\beta 1$ are the most studied integrin receptors because they are the partner receptors of many heterodimeric receptors. RGD and its derivative peptide sequences binding to $\alpha \mathrm{v}$ and $\beta 1$ units are the most studied ligands against integrins with therapeutic potential. However, most of them are not specific to one but various integrin receptors.

Furthermore, it is very important to realize that integrin $\alpha \mathrm{v}$ and $\beta 1$ are widely expressed within the human body and are not specifically expressed. Some of the cells types which express integrin $\alpha v$ and $\beta 1$ include e.g. endothelial cells, tumor cells. Therefore, molecules targeting $\alpha$ v or $\beta 1$ intergins are prone to exert off target effects. This could also explain why inhibitors such as Cilengitide, 
which have made it to phase III clinical trials have finally been terminated. Especially in fibrosis related diseases, it could be an interesting approach to develop molecules targeting integrins with a more disease-specific expression which are not involved in angiogenesis but for example expressed on myofibroblasts or tumor associates macrophages, two of the main drivers of disease progression in fibrosis, tumor stroma and wound healing. Therefore, studies exploiting the expression of a specific integrin subunit and its cellular localization within pathological tissues and other healthy organs are crucial to determine novel integrin-based therapeutic targets. Moreover, the design of integrin subunit specific drugs will decrease the risk of off-target effects due to a high specificity of these molecules. An additional major aspects for the identification of integrin subunits as therapeutic targets is to have a clear understanding of integrin subunit - growth factor receptor interactions.

Furthermore, with regard to drug targeting approaches, mainly targets of RGDbased targeting peptides binding to integrin $\alpha v \beta 3, \alpha v \beta 5, \alpha v \beta 6$ and $\alpha 5 \beta 1$ have been applied. Also in this context targeting of non-disease-specific integrins and integrin subunits can lead to off target effects and could results in severe toxicities, depending on the payload of the delivery system, even if the targeting molecule itself does not exert toxic effects.

Another important point to consider with regard to RGD-based targeting approaches is a finding by Kunjachan et al. ${ }^{200}$ which showed that passive targeting of $10 \mathrm{~nm}$-size polymeric nanocarriers resulted in enhanced intratumoral nanoparticle accumulation and retention when compared with RGD-peptide modified polymeric nanoparticles. These observations might be different for targeting systems with a larger size but it should be taken into consideration that targeting approaches directed towards targets with a more specific expression within a certain disease might increase site-specific accumulation and retention and additionally reduce side effects.

In conclusion, studies which have revealed the biological function of various integrins within fibrosis related disease, although not completely understood, imply various novel therapeutic and drug targeting targets. One of the major challenges remaining is to use the knowledge obtained on integrin biology to develop highly integrin subunit specific targeting molecules, which can be applied as therapeutics by themselves or utilized as targeting ligands in diseasespecific drug delivery systems. 


\subsection{References}

1 Mehal, W. Z., Iredale, J. \& Friedman, S. L. Scraping fibrosis: expressway to the core of fibrosis. Nature medicine 17, 552-553, doi:10.1038/nm0511-552 (2011).

2 Rybinski, B., Franco-Barraza, J. \& Cukierman, E. The wound healing, chronic fibrosis, and cancer progression triad. Physiological genomics 46, 223-244, doi:10.1152/physiolgenomics.00158.2013 (2014).

3 Henderson, N. C. \& Sheppard, D. Integrin-mediated regulation of TGFbeta in fibrosis. Biochimica et biophysica acta 1832, 891-896, doi:10.1016/j.bbadis.2012.10.005 (2013).

4 Conroy, K. P., Kitto, L. J. \& Henderson, N. C. alphav integrins: key regulators of tissue fibrosis. Cell and tissue research 365, 511-519, doi:10.1007/s00441-016-2407-9 (2016).

5 Seguin, L., Desgrosellier, J. S., Weis, S. M. \& Cheresh, D. A. Integrins and cancer: regulators of cancer stemness, metastasis, and drug resistance. Trends in cell biology 25, 234-240, doi:10.1016/j.tcb.2014.12.006 (2015).

6 Koivisto, L., Heino, J., Hakkinen, L. \& Larjava, H. Integrins in Wound Healing. Advances in wound care 3, 762-783, doi:10.1089/wound.2013.0436 (2014).

7 Danen, E. H. J. Integrin Signaling as a Cancer Drug Target. ISRN Cell Biology 2013, 14, doi:10.1155/2013/135164 (2013).

8 Desgrosellier, J. S. \& Cheresh, D. A. Integrins in cancer: biological implications and therapeutic opportunities. Nature reviews. Cancer 10, 9-22, doi:10.1038/nrc2748 (2010).

9 Berrier, A. L. \& Yamada, K. M. Cell-matrix adhesion. Journal of cellular physiology 213, 565-573, doi:10.1002/jcp.21237 (2007).

10 Legate, K. R., Montanez, E., Kudlacek, O. \& Fassler, R. ILK, PINCH and parvin: the tIPP of integrin signalling. Nature reviews. Molecular cell biology 7, 20-31, doi:10.1038/nrm1789 (2006).

11 Zoller, M. Tetraspanins: push and pull in suppressing and promoting metastasis. Nature reviews. Cancer 9, 40-55, doi:10.1038/nrc2543 (2009).

12 Giancotti, F. G. \& Tarone, G. Positional control of cell fate through joint integrin/receptor protein kinase signaling. Annual review of cell and developmental biology

19 , 173-206, doi:10.1146/annurev.cellbio.19.031103.133334 (2003). 
13 Ivaska, J. \& Heino, J. Cooperation between integrins and growth factor receptors in signaling and endocytosis. Annu Rev Cell Dev Biol 27, 291320, doi:10.1146/annurev-cellbio-092910-154017 (2011).

14 Cabodi, S. et al. Integrin regulation of epidermal growth factor (EGF) receptor and of EGF-dependent responses. Biochemical Society transactions 32, 438-442, doi:10.1042/BST0320438 (2004).

15 Schwartz, M. A. \& Ginsberg, M. H. Networks and crosstalk: integrin signalling spreads. Nature cell biology 4, E65-68, doi:10.1038/ncb0402e65 (2002).

16 Yamada, K. M. \& Even-Ram, S. Integrin regulation of growth factor receptors. Nature cell biology 4, E75-76, doi:10.1038/ncb0402-e75 (2002).

17 Ivaska, J. \& Heino, J. Interplay between cell adhesion and growth factor receptors: from the plasma membrane to the endosomes. Cell and tissue research 339, 111-120, doi:10.1007/s00441-009-0857-z (2010).

18 Moro, L. et al. Integrins induce activation of EGF receptor: role in MAP kinase induction and adhesion-dependent cell survival. The EMBO journal 17, 6622-6632, doi:10.1093/emboj/17.22.6622 (1998).

19 Moro, L. et al. Integrin-induced epidermal growth factor (EGF) receptor activation requires c-Src and p130Cas and leads to phosphorylation of specific EGF receptor tyrosines. The Journal of biological chemistry 277, 9405-9414, doi:10.1074/jbc.M109101200 (2002).

20 Danilkovitch-Miagkova, A. et al. Integrin-mediated RON growth factor receptor phosphorylation requires tyrosine kinase activity of both the receptor and c-Src. The Journal of biological chemistry 275, 1478314786, doi:10.1074/jbc.C000028200 (2000).

21 Borges, E., Jan, Y. \& Ruoslahti, E. Platelet-derived growth factor receptor beta and vascular endothelial growth factor receptor 2 bind to the beta 3 integrin through its extracellular domain. J Biol Chem 275, 39867-39873, doi:10.1074/jbc.M007040200 (2000).

22 DeMali, K. A., Balciunaite, E. \& Kazlauskas, A. Integrins enhance platelet-derived growth factor (PDGF)-dependent responses by altering the signal relay enzymes that are recruited to the PDGF beta receptor. $J$ Biol Chem 274, 19551-19558 (1999).

23 Schneller, M., Vuori, K. \& Ruoslahti, E. Alphavbeta3 integrin associates with activated insulin and PDGFbeta receptors and potentiates the biological activity of PDGF. EMBO $J$ 16, 5600-5607, doi:10.1093/emboj/16.18.5600 (1997). 
24 Soldi, R. et al. Role of alphavbeta3 integrin in the activation of vascular endothelial growth factor receptor-2. EMBO $J$ 18, 882-892, doi:10.1093/emboj/18.4.882 (1999).

25 Sundberg, C. \& Rubin, K. Stimulation of beta1 integrins on fibroblasts induces PDGF independent tyrosine phosphorylation of PDGF betareceptors. The Journal of cell biology 132, 741-752 (1996).

26 Wang, R., Ferrell, L. D., Faouzi, S., Maher, J. J. \& Bishop, J. M. Activation of the Met receptor by cell attachment induces and sustains hepatocellular carcinomas in transgenic mice. J Cell Biol 153, 10231034 (2001).

27 Chiu, S. J., Jiang, S. T., Wang, Y. K. \& Tang, M. J. Hepatocyte growth factor upregulates alpha2beta1 integrin in Madin-Darby canine kidney cells: implications in tubulogenesis. Journal of biomedical science $\mathbf{9}$, 261-272, doi:59427 (2002).

28 Liang, C. C. \& Chen, H. C. Sustained activation of extracellular signalregulated kinase stimulated by hepatocyte growth factor leads to integrin alpha 2 expression that is involved in cell scattering. The Journal of biological chemistry 276, 21146-21152, doi:10.1074/jbc.M010669200 (2001).

29 Chen, X. et al. Integrin-mediated type II TGF-beta receptor tyrosine dephosphorylation controls SMAD-dependent profibrotic signaling. The Journal of clinical investigation 124, 3295-3310, doi:10.1172/JCI71668 (2014).

30 Ruoslahti, E. \& Pierschbacher, M. D. New perspectives in cell adhesion: RGD and integrins. Science 238, 491-497 (1987).

31 Munger, J. S. et al. The integrin alpha $\mathrm{v}$ beta 6 binds and activates latent TGF beta 1: a mechanism for regulating pulmonary inflammation and fibrosis. Cell 96, 319-328 (1999).

$32 \mathrm{Mu}, \mathrm{D}$. et al. The integrin alpha(v)beta8 mediates epithelial homeostasis through MT1-MMP-dependent activation of TGF-beta1. The Journal of cell biology 157, 493-507, doi:10.1083/jcb.200109100 (2002).

33 Asano, Y. et al. Increased expression of integrin alpha(v)beta3 contributes to the establishment of autocrine TGF-beta signaling in scleroderma fibroblasts. J Immunol 175, 7708-7718 (2005).

34 Asano, Y., Ihn, H., Yamane, K., Jinnin, M. \& Tamaki, K. Increased expression of integrin alphavbeta5 induces the myofibroblastic differentiation of dermal fibroblasts. The American journal of pathology 168, 499-510 (2006). 
35 Wipff, P. J., Rifkin, D. B., Meister, J. J. \& Hinz, B. Myofibroblast contraction activates latent TGF-beta1 from the extracellular matrix. The Journal of cell biology 179, 1311-1323, doi:10.1083/jcb.200704042 (2007).

36 Prieto, A. L., Edelman, G. M. \& Crossin, K. L. Multiple integrins mediate cell attachment to cytotactin/tenascin. Proc. Natl. Acad. Sci. U. S. A. 90, 10154-10158 (1993).

37 Marsh, D. et al. alpha vbeta 6 Integrin promotes the invasion of morphoeic basal cell carcinoma through stromal modulation. Cancer research 68, 3295-3303, doi:10.1158/0008-5472.CAN-08-0174 (2008).

38 Galliher, A. J. \& Schiemann, W. P. Beta3 integrin and Src facilitate transforming growth factor-beta mediated induction of epithelialmesenchymal transition in mammary epithelial cells. Breast cancer research : BCR 8, R42, doi:10.1186/bcr1524 (2006).

39 Reynolds, L. E. et al. Accelerated re-epithelialization in beta3-integrindeficient- mice is associated with enhanced TGF-betal signaling. Nature medicine 11, 167-174, doi:10.1038/nm1165 (2005).

40 Asano, Y., Ihn, H., Jinnin, M., Mimura, Y. \& Tamaki, K. Involvement of alphavbeta5 integrin in the establishment of autocrine TGF-beta signaling in dermal fibroblasts derived from localized scleroderma. The Journal of investigative dermatology 126, 1761-1769, doi:10.1038/sj.jid.5700331 (2006).

41 Hood, J. D., Frausto, R., Kiosses, W. B., Schwartz, M. A. \& Cheresh, D. A. Differential alphav integrin-mediated Ras-ERK signaling during two pathways of angiogenesis. The Journal of cell biology 162, 933-943, doi:10.1083/jcb.200304105 (2003).

42 Friedlander, M. et al. Definition of two angiogenic pathways by distinct alpha v integrins. Science 270, 1500-1502 (1995).

43 Weis, S. M. et al. Cooperation between VEGF and beta3 integrin during cardiac vascular development. Blood 109, 1962-1970, doi:10.1182/blood-2005-10-038893 (2007).

44 Nikolopoulos, S. N., Blaikie, P., Yoshioka, T., Guo, W. \& Giancotti, F. G. Integrin beta4 signaling promotes tumor angiogenesis. Cancer cell $\mathbf{6}$, 471-483, doi:10.1016/j.ccr.2004.09.029 (2004).

45 Chen, $X$. et al. Integrin alphalbetal controls reactive oxygen species synthesis by negatively regulating epidermal growth factor receptormediated Rac activation. Molecular and cellular biology 27, 3313-3326, doi:10.1128/MCB.01476-06 (2007). 
46 Mattila, E. et al. Negative regulation of EGFR signalling through integrin-alpha1beta1-mediated activation of protein tyrosine phosphatase TCPTP. Nature cell biology 7, 78-85, doi:10.1038/ncb1209 (2005).

47 Mattila, E., Auvinen, K., Salmi, M. \& Ivaska, J. The protein tyrosine phosphatase TCPTP controls VEGFR2 signalling. Journal of cell science 121, 3570-3580, doi:10.1242/jcs.031898 (2008).

48 Bertotti, A., Comoglio, P. M. \& Trusolino, L. Beta4 integrin is a transforming molecule that unleashes Met tyrosine kinase tumorigenesis. Cancer research 65, 10674-10679, doi:10.1158/00085472.CAN-05-2827 (2005).

49 Zhu, C. Q. et al. Integrin alpha 11 regulates IGF2 expression in fibroblasts to enhance tumorigenicity of human non-small-cell lung cancer cells. Proc Natl Acad Sci U S A 104, 11754-11759, doi:10.1073/pnas.0703040104 (2007).

50 Huang, G. \& Brigstock, D. R. Integrin expression and function in the response of primary culture hepatic stellate cells to connective tissue growth factor (CCN2). Journal of cellular and molecular medicine 15, 1087-1095, doi:10.1111/j.1582-4934.2010.01072.x (2011).

51 Singer, A. J. \& Clark, R. A. Cutaneous wound healing. The New England journal of medicine 341, 738-746, doi:10.1056/NEJM199909023411006 (1999).

52 Liu, S. et al. Expression of integrin beta1 by fibroblasts is required for tissue repair in vivo. Journal of cell science 123, 3674-3682, doi:10.1242/jcs.070672 (2010).

53 Gardner, H. Integrin alphalbeta1. Advances in experimental medicine and biology 819, 21-39, doi:10.1007/978-94-017-9153-3_2 (2014).

54 Zeltz, C. \& Gullberg, D. The integrin-collagen connection - a glue for tissue repair? Journal of cell science 129, 1284, doi:10.1242/jcs.188672 (2016).

55 Ravanti, L., Heino, J., Lopez-Otin, C. \& Kahari, V. M. Induction of collagenase-3 (MMP-13) expression in human skin fibroblasts by threedimensional collagen is mediated by p38 mitogen-activated protein kinase. The Journal of biological chemistry 274, 2446-2455 (1999).

56 Wu, C., Chung, A. E. \& McDonald, J. A. A novel role for alpha 3 beta 1 integrins in extracellular matrix assembly. Journal of cell science $\mathbf{1 0 8}$ ( Pt 6), 2511-2523 (1995). 
57 Mitchell, K. et al. Alpha3beta1 integrin in epidermis promotes wound angiogenesis and keratinocyte-to-endothelial-cell crosstalk through the induction of MRP3. Journal of cell science 122, 1778-1787, doi:10.1242/jcs.040956 (2009).

58 Kato, A. et al. Dermatopontin interacts with fibronectin, promotes fibronectin fibril formation, and enhances cell adhesion. The Journal of biological chemistry 286, 14861-14869, doi:10.1074/jbc.M110.179762 (2011).

59 Bata-Csorgo, Z., Cooper, K. D., Ting, K. M., Voorhees, J. J. \& Hammerberg, C. Fibronectin and alpha5 integrin regulate keratinocyte cell cycling. A mechanism for increased fibronectin potentiation of $\mathrm{T}$ cell lymphokine-driven keratinocyte hyperproliferation in psoriasis. The Journal of clinical investigation 101, 1509-1518, doi:10.1172/JCI171 (1998).

60 Hakkinen, L., Heino, J., Koivisto, L. \& Larjava, H. Altered interaction of human granulation-tissue fibroblasts with fibronectin is regulated by alpha 5 beta 1 integrin. Biochimica et biophysica acta 1224, 33-42 (1994).

61 Lygoe, K. A., Wall, I., Stephens, P. \& Lewis, M. P. Role of vitronectin and fibronectin receptors in oral mucosal and dermal myofibroblast differentiation. Biology of the cell 99, 601-614, doi:10.1042/BC20070008 (2007).

62 Danussi, C. et al. EMILIN1-alpha4/alpha9 integrin interaction inhibits dermal fibroblast and keratinocyte proliferation. The Journal of cell biology 195, 131-145, doi:10.1083/jcb.201008013 (2011).

63 Nakayama, Y. et al. Blockade of interaction of alpha9 integrin with its ligands hinders the formation of granulation in cutaneous wound healing. Laboratory investigation; a journal of technical methods and pathology 90, 881-894, doi:10.1038/labinvest.2010.69 (2010).

64 Zeltz, C. \& Gullberg, D. The integrin-collagen connection--a glue for tissue repair? Journal of cell science 129, 653-664, doi:10.1242/jcs.180992 (2016).

65 Schulz, J. N. et al. Reduced granulation tissue and wound strength in the absence of alpha11beta1 integrin. The Journal of investigative dermatology 135, 1435-1444, doi:10.1038/jid.2015.24 (2015).

66 Zweers, M. C. et al. Integrin alpha2beta1 is required for regulation of murine wound angiogenesis but is dispensable for reepithelialization. 
The Journal of investigative dermatology 127, 467-478, doi:10.1038/sj.jid.5700546 (2007).

67 Sisco, M. et al. Delayed wound healing in Mac-1-deficient mice is associated with normal monocyte recruitment. Wound repair and regeneration : official publication of the Wound Healing Society [and] the European Tissue Repair Society 15, 566-571, doi:10.1111/j.1524475X.2007.00264.x (2007).

68 Margadant, C. \& Sonnenberg, A. Integrin-TGF-beta crosstalk in fibrosis, cancer and wound healing. EMBO reports 11, 97-105, doi:10.1038/embor.2009.276 (2010).

69 Pozzi, A. \& Zent, R. Integrins in kidney disease. Journal of the American Society of Nephrology : JASN 24, 1034-1039, doi:10.1681/ASN.2013010012 (2013).

70 Chen, X. et al. Lack of integrin alphalbeta1 leads to severe glomerulosclerosis after glomerular injury. The American journal of pathology 165, 617-630 (2004).

71 Borza, C. M. et al. Inhibition of integrin alpha2beta1 ameliorates glomerular injury. Journal of the American Society of Nephrology : JASN 23, 1027-1038, doi:10.1681/ASN.2011040367 (2012).

72 Rubel, D. et al. Collagen receptors integrin alpha2beta1 and discoidin domain receptor 1 regulate maturation of the glomerular basement membrane and loss of integrin alpha2beta1 delays kidney fibrosis in COL4A3 knockout mice. Matrix biology : journal of the International Society for Matrix Biology 34, 13-21, doi:10.1016/j.matbio.2014.01.006 (2014).

73 Bansal, R. et al. Integrin alpha 11 in the regulation of the myofibroblast phenotype: implications for fibrotic diseases. Experimental \& molecular medicine 49, e396, doi:10.1038/emm.2017.213 (2017).

$74 \mathrm{Hahm}, \mathrm{K}$. et al. Alphav beta6 integrin regulates renal fibrosis and inflammation in Alport mouse. The American journal of pathology 170, 110-125, doi:10.2353/ajpath.2007.060158 (2007).

75 Chang, Y. et al. Pharmacologic Blockade of alphavbeta1 Integrin Ameliorates Renal Failure and Fibrosis In Vivo. Journal of the American Society of Nephrology : JASN 28, 1998-2005, doi:10.1681/ASN.2015050585 (2017).

76 Hartner, A. et al. Tubulointerstitial de novo expression of the alpha8 integrin chain in a rodent model of renal fibrosis--a potential target for 
anti-fibrotic therapy? PLoS One 7, e48362, doi:10.1371/journal.pone.0048362 (2012).

77 Zheng, G. et al. alpha3 Integrin of Cell-Cell Contact Mediates Kidney Fibrosis by Integrin-Linked Kinase in Proximal Tubular E-Cadherin Deficient Mice. The American journal of pathology 186, 1847-1860, doi:10.1016/j.ajpath.2016.03.015 (2016).

78 Volpes, R., van den Oord, J. J. \& Desmet, V. J. Distribution of the VLA family of integrins in normal and pathological human liver tissue. Gastroenterology 101, 200-206 (1991).

79 Nejjari, M. et al. Integrin up-regulation in chronic liver disease: relationship with inflammation and fibrosis in chronic hepatitis $\mathrm{C}$. The Journal of pathology 195, 473-481, doi:10.1002/path.964 (2001).

80 Popov, Y. et al. Integrin alphavbeta6 is a marker of the progression of biliary and portal liver fibrosis and a novel target for antifibrotic therapies. Journal of hepatology 48, 453-464, doi:10.1016/j.jhep.2007.11.021 (2008).

81 Sacanella, E. et al. Upregulated expression of VLA proteins and CD29 in peripheral blood lymphocytes of chronic alcoholics without ethanolrelated diseases. Alcoholism, clinical and experimental research 23, 371-375 (1999).

82 Chedid, A. et al. Expression of the beta 1 chain (CD29) of integrins and CD45 in alcoholic liver disease. The VA Cooperative Study Group No. 275. The American journal of gastroenterology 88, 1920-1927 (1993).

83 Panasiuk, A., Zak, J., Maciorkowska, E., Panasiuk, B. \& Prokopowicz, D. Expression of beta2-integrin on leukocytes in liver cirrhosis. World journal of gastroenterology 12, 6193-6197 (2006).

84 Yasoshima, M., Nakanuma, Y., Tsuneyama, K., Van de Water, J. \& Gershwin, M. E. Immunohistochemical analysis of adhesion molecules in the micro-environment of portal tracts in relation to aberrant expression of PDC-E2 and HLA-DR on the bile ducts in primary biliary cirrhosis. The Journal of pathology 175, 319-325, doi:10.1002/path.1711750310 (1995).

85 Nadler, E. P. et al. Integrin alphavbeta6 and mediators of extracellular matrix deposition are up-regulated in experimental biliary atresia. The Journal of surgical research 154, 21-29, doi:10.1016/j.jss.2008.05.023 (2009).

86 Banerjee, A., Lee, J. H. \& Ramaiah, S. K. Interaction of osteopontin with neutrophil alpha(4)beta(1) and alpha(9)beta(1) integrins in a rodent 
model of alcoholic liver disease. Toxicology and applied pharmacology 233, 238-246, doi:10.1016/j.taap.2008.08.008 (2008).

87 Bautista, A. P. Chronic alcohol intoxication enhances the expression of CD18 adhesion molecules on rat neutrophils and release of a chemotactic factor by Kupffer cells. Alcoholism, clinical and experimental research 19, 285-290 (1995).

88 Shiina, M. et al. Up-regulation of CD11a (LFA-1) expression on peripheral CD4+ T cells in primary biliary cirrhosis. Digestive diseases and sciences 47, 1209-1215 (2002).

89 Gujral, J. S., Farhood, A., Bajt, M. L. \& Jaeschke, H. Neutrophils aggravate acute liver injury during obstructive cholestasis in bile ductligated mice. Hepatology 38, 355-363, doi:10.1053/jhep.2003.50341 (2003).

90 Kodali, P., Wu, P., Lahiji, P. A., Brown, E. J. \& Maher, J. J. ANIT toxicity toward mouse hepatocytes in vivo is mediated primarily by neutrophils via CD18. American journal of physiology. Gastrointestinal and liver physiology 291, G355-363, doi:10.1152/ajpgi.00458.2005 (2006).

91 Aspinall, A. I. et al. CX(3)CR1 and vascular adhesion protein-1dependent recruitment of CD16(+) monocytes across human liver sinusoidal endothelium. Hepatology 51, 2030-2039, doi:10.1002/hep.23591 (2010).

92 Milliano, M. T. \& Luxon, B. A. Initial signaling of the fibronectin receptor (alpha5beta1 integrin) in hepatic stellate cells is independent of tyrosine phosphorylation. Journal of hepatology 39, 32-37 (2003).

93 Levine, D. et al. Expression of the integrin alpha8beta1 during pulmonary and hepatic fibrosis. The American journal of pathology $\mathbf{1 5 6}$, 1927-1935 (2000).

94 Henderson, N. C. et al. Targeting of alphav integrin identifies a core molecular pathway that regulates fibrosis in several organs. Nature medicine 19, 1617-1624, doi:10.1038/nm.3282 (2013).

95 Busk, M., Pytela, R. \& Sheppard, D. Characterization of the integrin alpha v beta 6 as a fibronectin-binding protein. The Journal of biological chemistry 267, 5790-5796 (1992).

96 Breuss, J. M., Gillett, N., Lu, L., Sheppard, D. \& Pytela, R. Restricted distribution of integrin beta $6 \mathrm{mRNA}$ in primate epithelial tissues. The journal of histochemistry and cytochemistry : official journal of the Histochemistry Society 41, 1521-1527 (1993). 
97 Chen, H. et al. Mechanosensing by the alpha6-integrin confers an invasive fibroblast phenotype and mediates lung fibrosis. Nature communications 7, 12564, doi:10.1038/ncomms12564 (2016).

98 Araya, J. et al. Squamous metaplasia amplifies pathologic epithelialmesenchymal interactions in COPD patients. The Journal of clinical investigation 117, 3551-3562, doi:10.1172/JCI32526 (2007).

99 Araya, J., Cambier, S., Morris, A., Finkbeiner, W. \& Nishimura, S. L. Integrin-mediated transforming growth factor-beta activation regulates homeostasis of the pulmonary epithelial-mesenchymal trophic unit. The American journal of pathology 169, 405-415 (2006).

100 Kitamura, H. et al. Mouse and human lung fibroblasts regulate dendritic cell trafficking, airway inflammation, and fibrosis through integrin alphavbeta8-mediated activation of TGF-beta. The Journal of clinical investigation 121, 2863-2875, doi:10.1172/JCI45589 (2011).

101 Scotton, C. J. et al. Increased local expression of coagulation factor X contributes to the fibrotic response in human and murine lung injury. The Journal of clinical investigation 119, 2550-2563, doi:10.1172/JCI33288 (2009).

102 Kim, K. K. et al. Alveolar epithelial cell mesenchymal transition develops in vivo during pulmonary fibrosis and is regulated by the extracellular matrix. Proc. Natl. Acad. Sci. U. S. A. 103, 13180-13185, doi:10.1073/pnas.0605669103 (2006).

103 Kim, Y. et al. Integrin alpha3beta1-dependent beta-catenin phosphorylation links epithelial Smad signaling to cell contacts. The Journal of cell biology 184, 309-322, doi:10.1083/jcb.200806067 (2009).

104 Kim, K. K. et al. Epithelial cell alpha3beta1 integrin links beta-catenin and Smad signaling to promote myofibroblast formation and pulmonary fibrosis. The Journal of clinical investigation 119, 213-224, doi:10.1172/JCI36940 (2009).

105 Kuninty, P. R., Schnittert, J., Storm, G. \& Prakash, J. MicroRNA Targeting to Modulate Tumor Microenvironment. Front Oncol 6, 3, doi:10.3389/fonc.2016.00003 (2016).

106 Parajuli, H. et al. Integrin alpha11 is overexpressed by tumour stroma of head and neck squamous cell carcinoma and correlates positively with alpha smooth muscle actin expression. Journal of oral pathology \& medicine : official publication of the International Association of Oral 
Pathologists and the American Academy of Oral Pathology 46, 267-275, doi:10.1111/jop.12493 (2017).

107 Franco-Barraza, J. et al. Matrix-regulated integrin alphavbeta5 maintains alpha5beta1-dependent desmoplastic traits prognostic of neoplastic recurrence. eLife 6, doi:10.7554/eLife.20600 (2017).

108 Carracedo, S. et al. The fibroblast integrin alpha11beta1 is induced in a mechanosensitive manner involving activin $\mathrm{A}$ and regulates myofibroblast differentiation. The Journal of biological chemistry $\mathbf{2 8 5}$, 10434-10445, doi:10.1074/jbc.M109.078766 (2010).

109 Wang, K. K. et al. Novel candidate tumor marker genes for lung adenocarcinoma. Oncogene 21, 7598-7604, doi:10.1038/sj.onc.1205953 (2002).

110 Westcott, J. M. et al. An epigenetically distinct breast cancer cell subpopulation promotes collective invasion. The Journal of clinical investigation 125, 1927-1943, doi:10.1172/JCI77767 (2015).

111 Lu, N., Karlsen, T. V., Reed, R. K., Kusche-Gullberg, M. \& Gullberg, D. Fibroblast alpha11beta1 integrin regulates tensional homeostasis in fibroblast/A549 carcinoma heterospheroids. PLoS One 9, e103173, doi:10.1371/journal.pone.0103173 (2014).

112 Kale, S. et al. Osteopontin signaling upregulates cyclooxygenase-2 expression in tumor-associated macrophages leading to enhanced angiogenesis and melanoma growth via alpha9beta1 integrin. Oncogene 34, 5408-5410, doi:10.1038/onc.2015.315 (2015).

113 Zhou, W. et al. Periostin secreted by glioblastoma stem cells recruits M2 tumour-associated macrophages and promotes malignant growth. Nature cell biology 17, 170-182, doi:10.1038/ncb3090 (2015).

114 Weigelt, B., Peterse, J. L. \& van 't Veer, L. J. Breast cancer metastasis: markers and models. Nature reviews. Cancer 5, 591-602, doi:10.1038/nrc1670 (2005).

115 Ganguly, K. K., Pal, S., Moulik, S. \& Chatterjee, A. Integrins and metastasis. Cell adhesion \& migration 7, 251-261, doi:10.4161/cam.23840 (2013).

116 Ramirez, N. E. et al. The alpha(2)beta(1) integrin is a metastasis suppressor in mouse models and human cancer. The Journal of clinical investigation 121, 226-237, doi:10.1172/JCI42328 (2011).

117 Tran, T. et al. Loss of the alpha2beta1 integrin alters human papilloma virus-induced squamous carcinoma progression in vivo and in vitro. PLoS One 6, e26858, doi:10.1371/journal.pone.0026858 (2011). 
118 Bartolome, R. A. et al. Cadherin-17 interacts with alpha2beta1 integrin to regulate cell proliferation and adhesion in colorectal cancer cells causing liver metastasis. Oncogene 33, 1658-1669, doi:10.1038/onc.2013.117 (2014).

119 Matsuoka, T. et al. Increased expression of alpha2beta1-integrin in the peritoneal dissemination of human gastric carcinoma. International journal of molecular medicine 5, 21-25 (2000).

120 Baronas-Lowell, D. et al. Differential modulation of human melanoma cell metalloproteinase expression by alpha2beta1 integrin and CD44 triple-helical ligands derived from type IV collagen. The Journal of biological chemistry 279, 43503-43513, doi:10.1074/jbc.M405979200 (2004).

121 Hall, C. L., Dai, J., van Golen, K. L., Keller, E. T. \& Long, M. W. Type I collagen receptor (alpha 2 beta 1) signaling promotes the growth of human prostate cancer cells within the bone. Cancer research 66, 86488654, doi:10.1158/0008-5472.CAN-06-1544 (2006).

122 Berghoff, A. S. et al. alphavbeta3, alphavbeta5 and alphavbeta6 integrins in brain metastases of lung cancer. Clinical \& experimental metastasis 31, 841-851, doi:10.1007/s10585-014-9675-0 (2014).

123 Sun, C. et al. Association of CD98, integrin beta1, integrin beta3 and Fak with the progression and liver metastases of colorectal cancer. Pathology, research and practice 210, 668-674, doi:10.1016/j.prp.2014.06.016 (2014).

124 Hakkinen, L. et al. Increased expression of beta6-integrin in skin leads to spontaneous development of chronic wounds. The American journal of pathology 164, 229-242 (2004).

125 AlDahlawi, S., Eslami, A., Hakkinen, L. \& Larjava, H. S. The alphavbeta6 integrin plays a role in compromised epidermal wound healing. Wound repair and regeneration : official publication of the Wound Healing Society [and] the European Tissue Repair Society 14, 289-297, doi:10.1111/j.1743-6109.2006.00123.x (2006).

126 Clark, R. A., Ashcroft, G. S., Spencer, M. J., Larjava, H. \& Ferguson, M. W. Re-epithelialization of normal human excisional wounds is associated with a switch from alpha $\mathrm{v}$ beta 5 to alpha $\mathrm{v}$ beta 6 integrins. The British journal of dermatology 135, 46-51 (1996).

127 Haapasalmi, K. et al. Keratinocytes in human wounds express alpha $\mathrm{v}$ beta 6 integrin. The Journal of investigative dermatology 106, 42-48 (1996). 
128 Pilcher, B. K. et al. The activity of collagenase-1 is required for keratinocyte migration on a type I collagen matrix. The Journal of cell biology 137, 1445-1457 (1997).

129 Zhang, Z. et al. The alpha v beta 1 integrin functions as a fibronectin receptor but does not support fibronectin matrix assembly and cell migration on fibronectin. The Journal of cell biology 122, 235-242 (1993).

130 Widgerow, A. D. Chronic wounds - is cellular 'reception' at fault? Examining integrins and intracellular signalling. International wound journal 10, 185-192, doi:10.1111/j.1742-481X.2012.00967.x (2013).

131 Schulz, J. N. et al. Reduced granulation tissue and wound strength in the absence of alpha11beta1 integrin. The Journal of investigative dermatology 135, 1435-1444, doi:10.1038/jid.2015.24 (2015).

132 Frank, D. E. \& Carter, W. G. Laminin 5 deposition regulates keratinocyte polarization and persistent migration. Journal of cell science 117, 1351-1363, doi:10.1242/jcs.01003 (2004).

133 Grose, R. et al. A crucial role of beta 1 integrins for keratinocyte migration in vitro and during cutaneous wound repair. Development 129, 2303-2315 (2002).

134 Margadant, C. et al. Integrin alpha3beta1 inhibits directional migration and wound re-epithelialization in the skin. Journal of cell science $\mathbf{1 2 2}$, 278-288, doi:10.1242/jcs.029108 (2009).

135 Madamanchi, A. et al. Mitigation of oxygen-induced retinopathy in alpha2beta1 integrin-deficient mice. Investigative ophthalmology \& visual science 55, 4338-4347, doi:10.1167/iovs.14-14061 (2014).

136 Allen, P. B. et al. Review article: moving towards common therapeutic goals in Crohn's disease and rheumatoid arthritis. Alimentary pharmacology \& therapeutics 45, 1058-1072, doi:10.1111/apt.13995 (2017).

137 Gutheil, J. C. et al. Targeted antiangiogenic therapy for cancer using Vitaxin: a humanized monoclonal antibody to the integrin alphavbeta3. Clinical cancer research : an official journal of the American Association for Cancer Research 6, 3056-3061 (2000).

138 Delbaldo, C. et al. Phase I and pharmacokinetic study of etaracizumab (Abegrin), a humanized monoclonal antibody against alphavbeta3 integrin receptor, in patients with advanced solid tumors. Investigational new drugs 26, 35-43, doi:10.1007/s10637-007-9077-0 (2008). 
139 McNeel, D. G. et al. Phase I trial of a monoclonal antibody specific for alphavbeta3 integrin (MEDI-522) in patients with advanced malignancies, including an assessment of effect on tumor perfusion. Clinical cancer research : an official journal of the American Association for Cancer Research 11, 7851-7860, doi:10.1158/10780432.CCR-05-0262 (2005).

140 Hersey, P. et al. A randomized phase 2 study of etaracizumab, a monoclonal antibody against integrin alpha(v)beta(3), + or - dacarbazine in patients with stage IV metastatic melanoma. Cancer 116, 1526-1534, doi:10.1002/cncr.24821 (2010).

141 Mullamitha, S. A. et al. Phase I evaluation of a fully human anti-alphav integrin monoclonal antibody (CNTO 95) in patients with advanced solid tumors. Clinical cancer research : an official journal of the American Association for Cancer Research 13, 2128-2135, doi:10.1158/1078-0432.CCR-06-2779 (2007).

142 Heidenreich, A. et al. A randomized, double-blind, multicenter, phase 2 study of a human monoclonal antibody to human alphanu integrins (intetumumab) in combination with docetaxel and prednisone for the first-line treatment of patients with metastatic castration-resistant prostate cancer. Annals of oncology : official journal of the European Society for Medical Oncology 24, 329-336, doi:10.1093/annonc/mds505 (2013).

143 Uronis, H. E. et al. A Phase I/biomarker study of bevacizumab in combination with CNTO 95 in patients with advanced solid tumors. Cancer chemotherapy and pharmacology 75, 343-352, doi:10.1007/s00280-014-2647-x (2015).

144 Beekman, K. W. et al. Phase II evaluations of cilengitide in asymptomatic patients with androgen-independent prostate cancer: scientific rationale and study design. Clinical genitourinary cancer $\mathbf{4}$, 299-302, doi:10.3816/CGC.2006.n.012 (2006).

145 Nabors, L. B. et al. Phase I and correlative biology study of cilengitide in patients with recurrent malignant glioma. Journal of clinical oncology : official journal of the American Society of Clinical Oncology 25, 16511657, doi:10.1200/JCO.2006.06.6514 (2007).

146 Reardon, D. A. et al. Randomized phase II study of cilengitide, an integrin-targeting arginine-glycine-aspartic acid peptide, in recurrent glioblastoma multiforme. Journal of clinical oncology : official journal 
of the American Society of Clinical Oncology 26, 5610-5617, doi:10.1200/JCO.2008.16.7510 (2008).

147 MacDonald, T. J. et al. Phase I clinical trial of cilengitide in children with refractory brain tumors: Pediatric Brain Tumor Consortium Study PBTC-012. Journal of clinical oncology : official journal of the American Society of Clinical Oncology 26, 919-924, doi:10.1200/JCO.2007.14.1812 (2008).

148 Reed, N. I. et al. The alphavbeta1 integrin plays a critical in vivo role in tissue fibrosis. Science translational medicine 7, 288ra279, doi:10.1126/scitranslmed.aaa5094 (2015).

149 Horan, G. S. et al. Partial inhibition of integrin alpha(v)beta6 prevents pulmonary fibrosis without exacerbating inflammation. American journal of respiratory and critical care medicine 177, 56-65, doi:10.1164/rccm.200706-805OC (2008).

150 Puthawala, K. et al. Inhibition of integrin alpha(v)beta6, an activator of latent transforming growth factor-beta, prevents radiation-induced lung fibrosis. American journal of respiratory and critical care medicine 177, 82-90, doi:10.1164/rccm.200706-806OC (2008).

151 Wang, B. et al. Role of alphavbeta6 integrin in acute biliary fibrosis. Hepatology 46, 1404-1412, doi:10.1002/hep.21849 (2007).

152 Sullivan, B. P., Weinreb, P. H., Violette, S. M. \& Luyendyk, J. P. The coagulation system contributes to alphaVbeta6 integrin expression and liver fibrosis induced by cholestasis. The American journal of pathology 177, 2837-2849, doi:10.2353/ajpath.2010.100425 (2010).

153 Ma, L. J. et al. Transforming growth factor-beta-dependent and independent pathways of induction of tubulointerstitial fibrosis in beta6(-/-) mice. The American journal of pathology 163, 1261-1273 (2003).

154 https://clinicaltrials.gov/ct2/show/NCT01371305. STX-100 in Patients With Idiopathic Pulmonary Fibrosis (IPF), 2017).

155 Asano, Y., Ihn, H., Yamane, K., Kubo, M. \& Tamaki, K. Increased expression levels of integrin alphavbeta5 on scleroderma fibroblasts. The American journal of pathology 164, 1275-1292 (2004).

156 Asano, Y. et al. Involvement of alphavbeta5 integrin-mediated activation of latent transforming growth factor beta1 in autocrine transforming growth factor beta signaling in systemic sclerosis fibroblasts. Arthritis and rheumatism 52, 2897-2905, doi:10.1002/art.21246 (2005). 
157 Lygoe, K. A., Norman, J. T., Marshall, J. F. \& Lewis, M. P. AlphaV integrins play an important role in myofibroblast differentiation. Wound repair and regeneration : official publication of the Wound Healing Society [and] the European Tissue Repair Society 12, 461-470, doi:10.1111/j.1067-1927.2004.12402.x (2004).

158 Brooks, P. C., Clark, R. A. \& Cheresh, D. A. Requirement of vascular integrin alpha v beta 3 for angiogenesis. Science 264, 569-571 (1994).

159 Patsenker, E. et al. Inhibition of integrin alphavbeta6 on cholangiocytes blocks transforming growth factor-beta activation and retards biliary fibrosis progression. Gastroenterology 135, 660-670, doi:10.1053/j.gastro.2008.04.009 (2008).

160 Ellerby, H. M. et al. Anti-cancer activity of targeted pro-apoptotic peptides. Nature medicine 5, 1032-1038, doi:10.1038/12469 (1999).

161 Zhou, X. et al. Engagement of alphavbeta3 integrin regulates proliferation and apoptosis of hepatic stellate cells. The Journal of biological chemistry 279, 23996-24006, doi:10.1074/jbc.M311668200 (2004).

162 Patsenker, E. et al. Pharmacological inhibition of integrin alphavbeta3 aggravates experimental liver fibrosis and suppresses hepatic angiogenesis. Hepatology 50, 1501-1511, doi:10.1002/hep.23144 (2009).

163 Muhanna, N. et al. Activation of hepatic stellate cells after phagocytosis of lymphocytes: A novel pathway of fibrogenesis. Hepatology 48, 963977, doi:10.1002/hep.22413 (2008).

164 Ulmasov, B. et al. Inhibitors of Arg-Gly-Asp-Binding Integrins Reduce Development of Pancreatic Fibrosis in Mice. Cellular and molecular gastroenterology and hepatology 2, 499-518, doi:10.1016/j.jcmgh.2016.03.004 (2016).

165 Ricart, A. D. et al. Volociximab, a chimeric monoclonal antibody that specifically binds alpha5beta1 integrin: a phase I, pharmacokinetic, and biological correlative study. Clinical cancer research : an official journal of the American Association for Cancer Research 14, 79247929, doi:10.1158/1078-0432.CCR-08-0378 (2008).

166 Barton, J. A multicenter phase II study of volociximab in patients with relapsed metastatic melanoma. Journal of Clinical Oncology 26, 90519051, doi:10.1200/jco.2008.26.15_suppl.9051 (2008).

167 Figlin, R. A., Kondagunta, G. V., Yazji, S., Motzer, R. J. \& Bukowski, R. M. Phase II study of volociximab (M200), an $\alpha 5 \beta 1$ anti-integrin 
antibody in refractory metastatic clear cell renal cell cancer (RCC). Journal of Clinical Oncology 24, 4535-4535, doi:10.1200/jco.2006.24.18_suppl.4535 (2006).

168 Evans, T. et al. Final results from cohort 1 of a phase II study of volociximab, an anti- $\alpha 5 \beta 1$ integrin antibody, in combination with gemcitabine (GEM) in patients (pts) with metastatic pancreatic cancer (MPC). Journal of Clinical Oncology 25, 4549-4549, doi:10.1200/jco.2007.25.18_suppl.4549 (2007).

169 Bell-McGuinn, K. M. et al. A phase II, single-arm study of the antialpha5beta1 integrin antibody volociximab as monotherapy in patients with platinum-resistant advanced epithelial ovarian or primary peritoneal cancer. Gynecologic oncology 121, 273-279, doi:10.1016/j.ygyno.2010.12.362 (2011).

170 Vergote, I. B. et al. Phase II study comparing volociximab (an antiangiogenic antibody) and pegylated liposomal doxorubicin (PLD) with PLD alone in recurrent ovarian or primary peritoneal cancer. Journal of Clinical Oncology 27, 5560-5560, doi:10.1200/jco.2009.27.15s.5560 (2009).

171 Besse, B. et al. Phase Ib safety and pharmacokinetic study of volociximab, an anti-alpha5beta1 integrin antibody, in combination with carboplatin and paclitaxel in advanced non-small-cell lung cancer. Annals of oncology : official journal of the European Society for Medical Oncology 24, 90-96, doi:10.1093/annonc/mds281 (2013).

172 Williams, R. Discontinued in 2013: oncology drugs. Expert opinion on investigational drugs 24, 95-110, doi:10.1517/13543784.2015.971154 (2015).

173 Kotoh, K. et al. Arg-Gly-Asp (RGD) peptide ameliorates carbon tetrachloride-induced liver fibrosis via inhibition of collagen production and acceleration of collagenase activity. International journal of molecular medicine 14, 1049-1053 (2004).

174 Khalili, P. et al. A non-RGD-based integrin binding peptide (ATN-161) blocks breast cancer growth and metastasis in vivo. Molecular cancer therapeutics 5, 2271-2280, doi:10.1158/1535-7163.MCT-06-0100 (2006).

175 Cianfrocca, M. E. et al. Phase 1 trial of the antiangiogenic peptide ATN161 (Ac-PHSCN-NH(2)), a beta integrin antagonist, in patients with solid tumours. British journal of cancer 94, 1621-1626, doi:10.1038/sj.bjc.6603171 (2006). 
176 Mita, M. et al. Phase I study of E7820, an oral inhibitor of integrin alpha2 expression with antiangiogenic properties, in patients with advanced malignancies. Clinical cancer research : an official journal of the American Association for Cancer Research 17, 193-200, doi:10.1158/1078-0432.CCR-10-0010 (2011).

177 A Phase II Study of the Safety and Efficacy of E7820 Plus Cetuximab in Colorectal Cancer, Preceded by a Run-in Study in Advanced Solid Tumors, 2006).

178 Carbonell, W. S., DeLay, M., Jahangiri, A., Park, C. C. \& Aghi, M. K. beta1 integrin targeting potentiates antiangiogenic therapy and inhibits the growth of bevacizumab-resistant glioblastoma. Cancer research 73, 3145-3154, doi:10.1158/0008-5472.CAN-13-0011 (2013).

179 Marcinkiewicz, C. et al. Obtustatin: a potent selective inhibitor of alphalbeta1 integrin in vitro and angiogenesis in vivo. Cancer research 63, 2020-2023 (2003).

180 Dold, S., Laschke, M. W., Lavasani, S., Menger, M. D. \& Thorlacius, $\mathrm{H}$. Cholestatic liver damage is mediated by lymphocyte function antigen-1-dependent recruitment of leukocytes. Surgery 144, 385-393, doi:10.1016/j.surg.2008.05.010 (2008).

181 Shi, C. et al. Monocyte trafficking to hepatic sites of bacterial infection is chemokine independent and directed by focal intercellular adhesion molecule-1 expression. J Immunol 184, 6266-6274, doi:10.4049/jimmunol.0904160 (2010).

182 Stupp, R. et al. Cilengitide combined with standard treatment for patients with newly diagnosed glioblastoma with methylated MGMT promoter (CENTRIC EORTC 26071-22072 study): a multicentre, randomised, open-label, phase 3 trial. The Lancet. Oncology 15, 11001108, doi:10.1016/S1470-2045(14)70379-1 (2014).

183 Arosio, D. \& Casagrande, C. Advancement in integrin facilitated drug delivery. Advanced drug delivery reviews 97, 111-143, doi:10.1016/j.addr.2015.12.001 (2016).

184 Arap, W., Pasqualini, R. \& Ruoslahti, E. Cancer treatment by targeted drug delivery to tumor vasculature in a mouse model. Science 279, 377380 (1998).

185 de Groot, F. M. et al. Design, synthesis, and biological evaluation of a dual tumor-specific motive containing integrin-targeted plasmincleavable doxorubicin prodrug. Molecular cancer therapeutics 1, 901911 (2002). 
186 Arosio, D. et al. Functionalized cyclic RGD peptidomimetics: conjugable ligands for alphavbeta3 receptor imaging. Bioconjugate chemistry 20, 1611-1617, doi:10.1021/bc900155j (2009).

187 Curnis, F. et al. IsoDGR-tagged albumin: a new alphavbeta3 selective carrier for nanodrug delivery to tumors. Small 9, 673-678, doi:10.1002/smll.201202310 (2013).

188 Stoeltzing, O. et al. Inhibition of integrin alpha5beta1 function with a small peptide (ATN-161) plus continuous 5-FU infusion reduces colorectal liver metastases and improves survival in mice. International journal of cancer 104, 496-503, doi:10.1002/ijc.10958 (2003).

189 Goodman, S. L. \& Picard, M. Integrins as therapeutic targets. Trends in pharmacological sciences 33, 405-412, doi:10.1016/j.tips.2012.04.002 (2012).

190 Dai, W. et al. PHSCNK-Modified and doxorubicin-loaded liposomes as a dual targeting system to integrin-overexpressing tumor neovasculature and tumor cells. Journal of drug targeting 18, 254-263, doi:10.3109/10611860903353354 (2010).

191 Mardilovich, A. \& Kokkoli, E. Biomimetic peptide-amphiphiles for functional biomaterials: the role of GRGDSP and PHSRN. Biomacromolecules 5, 950-957, doi:10.1021/bm0344351 (2004).

192 Pangburn, T. O., Georgiou, K., Bates, F. S. \& Kokkoli, E. Targeted polymersome delivery of siRNA induces cell death of breast cancer cells dependent upon Orai3 protein expression. Langmuir : the ACS journal of surfaces and colloids 28, 12816-12830, doi:10.1021/la300874z (2012).

193 Adil, M. M., Erdman, Z. S. \& Kokkoli, E. Transfection mechanisms of polyplexes, lipoplexes, and stealth liposomes in alpha(5)beta(1) integrin bearing DLD-1 colorectal cancer cells. Langmuir : the ACS journal of surfaces and colloids 30, 3802-3810, doi:10.1021/la5001396 (2014).

194 Wang, D. et al. Targeted iron-oxide nanoparticle for photodynamic therapy and imaging of head and neck cancer. ACS nano 8, 6620-6632, doi:10.1021/nn501652j (2014).

195 Rechenmacher, F. et al. Functionalizing alphavbeta3- or alpha5beta1selective integrin antagonists for surface coating: a method to discriminate integrin subtypes in vitro. Angewandte Chemie 52, 15721575, doi:10.1002/anie.201206370 (2013). 
196 Neubauer, S. et al. Selective imaging of the angiogenic relevant integrins alpha5beta1 and alphavbeta3. Angewandte Chemie 52, 11656-11659, doi:10.1002/anie.201306376 (2013).

197 Mukhopadhyay, S. et al. Conjugated platinum(IV)-peptide complexes for targeting angiogenic tumor vasculature. Bioconjugate chemistry 19, 39-49, doi:10.1021/bc070031k (2008).

198 Pan, D. et al. Antiangiogenic nanotherapy with lipase-labile Sn-2 fumagillin prodrug. Nanomedicine 7, 1507-1519, doi:10.2217/nnm.12.27 (2012).

199 Fu, X., Rivera, A., Tao, L. \& Zhang, X. Genetically modified T cells targeting neovasculature efficiently destroy tumor blood vessels, shrink established solid tumors and increase nanoparticle delivery. International journal of cancer 133, 2483-2492, doi:10.1002/ijc.28269 (2013).

200 Kunjachan, S. et al. Passive versus active tumor targeting using RGDand NGR-modified polymeric nanomedicines. Nano letters 14, 972-981, doi:10.1021/nl404391r (2014). 


\title{
Chapter 6 - Integrin $\alpha 11$ regulates differentiation of pancreatic stellate cells in pancreatic cancer
}

Jonas Schnittert et al.

Department of Biomaterials, Science and Technology, Section: Targeted Therapeutics, TechMed Centre, Faculty of Science and Technology, University of Twente, The Netherlands; BV, Enschede, The Netherlands.

Manuscript in preparation.

\begin{abstract}
:
Pancreatic ductal adenocarcinoma (PDAC) is the deadliest tumor due to its highly abundant tumor stroma. One of the main components of the tumor stroma are pancreatic stellate cells (PSCs), precursor cells of cancer-associated fibroblasts (CAFs), which support PDAC progression by enhancing tumor cell growth, invasion and metastasis. Integrins are extracellular matrix receptors playing a crucial role in cell adhesion, migration and function of several growth factors. In the presented study, we aimed to investigate the expression of integrin alpha11 (ITGA11) in human PDAC and study the role of ITGA11 in PSCs activation and function.
\end{abstract}

In this study, we show the immunohistological localization of ITGA11 for the first time in human PDAC specimens. We found that ITGA11 is highly expressed on stromal myofibroblasts of PDAC patients, as confirmed by colocalization with the myofibroblast marker alpha smooth muscle actin ( $\alpha-S M A)$. In contrast, there was no expression in healthy pancreas and other human organs. Activation of primary human PSCs (hPSCs) with TGF- $\beta$ or conditioned medium from Panc-1 (Panc-1 CM) resulted in the significant upregulation of ITGA11 and various markers which were previously shown to be upregulated in activated hPSCs and CAFs (e.g. $\alpha$-SMA, Collagen-I). Stable ITGA11 knockdown (shITGA11), significantly inhibited TGF- $\beta$ and Panc-1 CM mediated induction of major hPSC activation markers (such as $\alpha$-SMA, Collagen-I) on gene and protein level. Strikingly, various cytokines, growth factors, cell adhesion molecules and ECM components which were induced with TGF- $\beta$ or Panc-1 CM in hPSCs, were reduced in shITGA11 hPSCs. These findings demonstrate that these cytokines and growth factors (e.g. MIF, TGF- 33 , IGFBP-1, 3, CXCL-1, PDGF-BB) are regulated by ITGA11 upon TGF- $\beta$ or Panc-1 CM activation. 
Additionally, shITGA11 hPSCs had lowered migration and contractility. To study the effect of ITGA11 on the paracrine activity of hPSCs, we applied conditioned medium from hPSCs on Panc-1 tumor cells. We found that the conditioned medium obtained from TGF- $\beta$-activated hPSCs induced the migration and invasion ability of Panc- 1 . These inductions were highly reduced when the conditioned medium from shITGA11 hPSCs was used, demonstrating that ITGA11 knockdown can inhibit pro-tumoral paracrine signalling between hPSC and Panc-1 tumor cells.

In conclusion, this study presents ITGA11 as an interesting therapeutic target which is highly and specifically expressed in the pancreatic tumor stroma and plays a key role in the regulation of activation and paracrine function of hPSCs.

\subsection{Introduction}

Pancreatic ductal adenocarcinoma (PDAC) is one of the most devastating disease in our society with a 5 -year survival rate of only $8 \%{ }^{1}$. Due to the advanced stage of the disease, a surgical resection of the tumor is not feasible in most of the patients with PDAC. Therefore, radiotherapy or cytotoxic chemotherapy are the golden standard treatment, but these treatments are not effective for PDAC which results in a survival benefit of only a few months ${ }^{2,3}$. One of the biological features that contributes to the high drug resistance, progression, invasion and metastasis of PDAC is the abundant desmoplastic reaction, also known as the tumor stroma ${ }^{4}$. The pancreatic tumor stroma can take up to $90 \%$ of the entire tumor mass and its main cellular component are cancer-associated fibroblasts (CAFs) which support PDAC progression by producing ECM proteins and the secretion of various cytokines and growth factors, which stimulate tumor growth, angiogenesis, invasion, and metastases ${ }^{5-7}$.

Different studies have shown an anti-tumoral effects in pancreatic cancer by modulating its tumor stroma ${ }^{8,9}$, while on the contrary complete genetic depletion of the stroma resulted in an aggressive tumor phenotype ${ }^{10}$. Therefore, current strategies focus on modulating the activity of tumor stroma instead of completely depleting it to gain therapeutic benefits ${ }^{8,11-13}$. In human pancreatic cancer, the main progenitors of CAFs are human pancreatic stellate cells (hPSCs) ${ }^{14}$. In the healthy pancreas quiescent hPSCs have a cytoplasmic lipid storing capacity and are normally present in the connective tissue in low numbers, secreting only small amounts of ECM components ${ }^{15}$. However, during malignancy quiescent hPSCs get activated, lose their cytoplasmic lipid storing capacity and secret high amounts of ECM, which ultimately presents as a barrier for tumor drug 
penetration. In addition, activated hPSCs secrete various cytokines and growth factors which stimulate tumor cells and other stromal cells inducing tumor progression, metastasis and drug resistance ${ }^{16,17}$. Activated hPSCs therefore present an attractive and promising cellular target for developing therapies to modulate tumor stroma.

Cellular interaction with the extracellular environment are mostly mediated by integrins, a known family of cell surface receptors, which have been recognized for their role in myofibroblast differentiation ${ }^{18}$. In general, the integrin family consists of 24 different heterodimeric transmembrane receptors composed of different combinations of 18 different $\alpha$ - and 8 different $\beta$-subunits ${ }^{18}$. Since integrins build a physical connection with the inside and the outside of a cell, they are capable of conducting bidirectional signaling. Through this dynamic mechanism, integrins have the ability to control cellular cytoskeleton organization, thereby controlling crucial cellular functions such as cell adhesion, migration, proliferation, survival and differentiation ${ }^{18,19}$. Integrins are also known for their interaction with growth factors, for example, $\alpha \mathrm{v}$ integrins can activate latent TGF $\beta 1{ }^{18,20}$. Additionally, integrins interact with growth factor receptors via different mechanisms to regulate their signaling pathways ${ }^{18,21}$.

Integrins are therefore interesting target receptors to develop novel therapeutic strategies against tumor stroma. As collagens are the major ECM proteins in the tumor stroma, one of the major collagen binding integrin $\alpha 11 \beta 1$ (ITGA11) is an important receptor in CAFs. ITGA11 has been shown to be overexpressed in the tumor stroma of head and neck squamous cell carcinoma, in which it positively correlated with the expression of CAF marker $\alpha$-smooth muscle actin ( $\alpha$-SMA) ${ }^{22}$. Additionally, ITGA11 was recognized for its role in inducing tumor cell growth and metastatic potential of small cell lung carcinoma cells ${ }^{23}$. In this study, we investigated the expression and cellular localization of ITGA11 in human PDAC samples. Next, we studied the ITGA11 expression in activated hPSCs in vitro and demonstrated its role in regulating hPSC activation, differentiation, migration and phenotypic changes using knockdown studies. Finally, we investigated the influence of ITGA11 knockdown on hPSC-mediated paracrine effect on tumor cells. 


\subsection{Materials and Methods}

\subsubsection{Staining of human PDAC sections}

Paraffin embedded human PDAC sections and normal human pancreatic sections were obtained from LabPON (Hengelo, The Netherlands). Tissue sections were sliced, deparaffinized in xylene and rehydrated in serially diluted alcohol solutions, followed by demineralized water for 5 minutes respectively. Antigen retrieval was performed in Tris EDTA buffer ( $\mathrm{pH}$ 9.0, Dako, Glastrup, Denmark) by heat induction at $80^{\circ} \mathrm{C}$. Endogenous peroxidase was blocked by incubation in $0.3 \%$ hydrogen peroxide (Thermofisher Scientific, Landsmeer, The Netherlands) in methanol for $30 \mathrm{~min}$.

After rinsing with MilliQ water the tissue sections were incubated overnight at $4^{\circ} \mathrm{C}$ with primary antibodies (Table 1) diluted in PBS. Control samples were incubated without primary antibodies in PBS overnight at $4{ }^{\circ} \mathrm{C}$. The slides were then incubated with the secondary and tertiary fluorescent antibody or HRPlabeled antibody (Table 1) diluted in PBS supplemented with 5\% of normal human serum (Thermofisher Scientific), respectively for 1 hour at room temperature (RT). Stainings performed with HRP-labeled antibodies were visualized using 3.3-diaminobenzidine tetrahydrochloride solution (Dako) for 510 min resulting in brown color and then counterstained with hematoxylin for 2 min. Subsequently, the tissue sections were dehydrated and mounted with VectaMount (Vector, Burlingame, USA). For staining with fluorescent antibodies, the slides were washed and mounted with fluoroshield ${ }^{\mathrm{TM}}$ with DAPI (Sigma Aldrich) for staining of nuclei. All stained sections have been imaged using a Hamamatsu NanoZoomer Digital slide scanner 2.0HT (Hamamatsu Photonics, Bridgewater NJ). Scoring was performed visually.

Table 1. Antibodies used for the staining of human PDAC and normal pancreas sections.

\begin{tabular}{|l|l|l|}
\hline Antibody & Source & Dilution \\
\hline Goat anti-Integrin $\alpha 11$ & R\&D Systems & $1: 250$ \\
\hline Mouse anti- $\alpha$-SMA & Sigma Aldrich & $1: 200$ \\
\hline HRP-conjugated goat anti-rabbit IgG & DAKO & $1: 100$ \\
\hline HRP-conjugated rabbit anti-goat IgG & DAKO & $1: 100$ \\
\hline HRP-conjugated rabbit anti-mouse IgG & DAKO & $1: 100$ \\
\hline Alexa Fluor 594 labelled donkey anti-goat & Life Technologies & $1: 100$ \\
\hline Alexa Fluor 488 labelled donkey anti-mouse & Life Technologies & $1: 100$ \\
\hline
\end{tabular}




\subsubsection{Cell lines}

Primary human pancreatic stellate cells (ScienCell, Carlsbad, USA), were cultured in complete Stellate Cell Medium (supplemented with $2 \%$ FBS, $1 \%$ Penicillin/Streptomycin and $1 \%$ Stellate Cell Growth Supplements (SteCGS)) (ScienCell). Panc-1 cancer cells were cultured in Dulbecco's Modified Eagles medium (DMEM) High Glucose (4.5 g/l) with L-Glutamine (GE Healthcare, Vienna, Austria) supplemented with $10 \%$ FBS (Lonza, Verviers, Belgium), 100 $\mu \mathrm{g} / \mathrm{ml}$ penicillin/streptomycin (Sigma Aldrich, Zwijndrecht, The Netherlands). The cells were maintained at $37^{\circ} \mathrm{C}$ in a humidified 5\% CO2 atmosphere.

\subsubsection{Stable ITGA11 knockdown in hPSCs}

To generate hPSCs with a stable knockdown of ITGA11 (shITGA11), primary hPSCs were transfected with either a scrambled shRNA control plasmid or shRNA ITGA11 plasmid using lipofectamine 2000 (Invitrogen, Carlsbad, USA) as described elsewhere ${ }^{24}$. After 24 hours of transfection with lipofectamine, transfected cells were selected and cultured with $1 \mu \mathrm{g} / \mathrm{ml}$ of puromycin (Invitrogen).

\subsubsection{Quantitative real-time PCR and $\mathrm{RT}^{\mathbf{2}}$ profiler PCR array}

To evaluate the effect of ITGA11 knockdown on TGF- $\beta$-mediated hPSCs activation, cells were seeded into a 12 well plate at a seeding density of 40.000 cells/ well. The cells were starved for $24 \mathrm{~h}$ and activated with $5 \mathrm{ng} / \mu \mathrm{l} \mathrm{TGF}-\beta$ or Panc-1 conditioned medium. $24 \mathrm{~h}$ after activation, total RNA was isolated using the GenElute ${ }^{\mathrm{TM}}$ Mammalian Total RNA Miniprep Kit (Sigma Aldrich). The amount of isolated RNA was measured by a NanoDrop ${ }^{\circledR}$ ND-1000 Spectrophotometer (Thermo Scientific, Rockford, USA). Next, cDNA was synthesized with iScript ${ }^{\mathrm{TM}}$ cDNA Synthesis Kit (BioRad, Veenendaal, The Netherlands). $5 \mathrm{ng}$ cDNA were used for each real-time PCR reaction. Real-time PCR primers (Table 2) were purchased from (Sigma Aldrich). Real-time PCR was performed using the 2x SensiMix SYBR and Fluorescein Kit (Bioline GmbH, Luckenwalde, Germany) with a BioRad CFX384 Real-Time PCR detection system (BioRad). For the $\mathrm{RT}^{2}$ profiler PCR array for human extracellular matrix \& adhesion molecules (Qiagen, Hilden, Germany), cDNA from three independent experiments was mixed and performed as described in the manufacturer's instructions and read with a BioRad CFX384 Real-Time PCR detection system (BioRad). 
Table 2. Sequences of forward and reverse primers used during real-time PCR.

\begin{tabular}{|l|l|l|}
\hline Gene & Forward Primer & Reverse Primer \\
\hline RPS18 & TGAGGTGGAACGTGTGATCA & CCTCTATGGGCCCGAATCTT \\
\hline ITGA11 & CAGCTCGCTGGAGAGATACG & TTACAGGACGTGTTCGCCTC \\
\hline Acta-2 & CCCCATCTATGAGGGCTATG & CAGTGGCCATCTCATTTTCA \\
\hline ITGA11 & CAGCTCGCTGGAGAGATACG & TTACAGGACGTGTTCGCCTC \\
\hline PDGFßR & AGGCAAGCTGGTCAAGATCT & GCTGTTGAAGATGCTCTCCG \\
\hline Desmin & GCGGGTTTCGGCTCTAAGG & AGAAACTCCTGGTTCACCGC \\
\hline Collagen3 $\alpha 1$ & AAGAAGGCCCTGAAGCTGAT & GTGTTTCGTGCAACCATCCT \\
\hline NSDHL & ATGCTATGGAGAGGACCGTG & CAGAAGGAGTCACAGGCTCA \\
\hline CTGF & GTTTGGCCCAGACCCAACTA & GGCTCTGCTTCTCTAGCCTG \\
\hline INSIG1 & GGCAGCTTCCCAAGTATTCG & CTACCTCCTTTGGGCACTGA \\
\hline IDI1 & TTGGGCTGGATAAAACCCCT & GCAACATCCGGCATAACTGT \\
\hline MMP2 & AGGAGGAGAAGGCTGTGTTC & CTCCAGTTAAAGGCGGCATC \\
\hline CXCR-4 & GCGGTTACCATGGAGGGGAT & CCCATGACCAGGATGACCAAT \\
\hline
\end{tabular}

\subsubsection{Western blotting analysis}

Cells were prepared as described for real-time PCR analysis above. Cells were lysed using 1x SDS-lysis buffer. The cell lysis was centrifuged at 10,000 $\mathrm{g}$ for $10 \mathrm{~min}$ and the supernatant was collected for Western Blot analysis. In short, protein lysates and conditioned medium was loaded on $10 \%$ Tris-Glycine gels (Thermo Scientific) and transferred onto PVDF membranes (Thermo Scientific). The membranes were incubated overnight at $4{ }^{\circ} \mathrm{C}$ with the required primary antibody (Table 3), followed by incubation with species specific horseradish peroxidase (HRP) conjugated secondary and tertiary antibody for 1 hour at RT. HRP was detected with Pierce ${ }^{\mathrm{TM}}$ ECL Plus Western Blotting Substrate kit (Thermo Scientific) and exposed to FluorChem ${ }^{\mathrm{TM}}$ M System (ProteinSimple, San José, USA). The protein levels were quantified by Image J Software (NIH, $\mathrm{MD)}$.

Table 3. Primary and secondary antibodies used for western blot.

\begin{tabular}{|l|l|l|}
\hline Antibody & Source & Dilution \\
\hline Goat-anti-Integrin $\alpha 11$ & R\&D Systems & $1: 250$ \\
\hline Mouse anti- $\alpha$-SMA & Sigma Aldrich & $1: 200$ \\
\hline Mouse monoclonal anti- $\beta$-actin & Sigma Aldrich & $1: 10000$ \\
\hline HRP-conjugated goat anti-rabbit IgG & DAKO & $1: 2000$ \\
\hline HRP-conjugated rabbit anti-goat IgG & DAKO & $1: 2000$ \\
\hline HRP-conjugated rabbit anti-mouse IgG & DAKO & $1: 1000$ \\
\hline
\end{tabular}




\subsubsection{F-actin staining}

Cells were prepare as described above for quantitative real-time PCR. Subsequently the cells were fixed in PBS containing 10\% formaldehyde (Sigma Aldrich) for $15 \mathrm{~min}$. After permeabilization with 0.1 M Triton X-100 (Sigma Aldrich) for $5 \mathrm{~min}$, F-actin was stained with phalloidin (Life Technologies, Carlsbad, USA) at a concentration of $250 \mathrm{ng} / \mathrm{ml}$ for 30 minutes at RT. Next, cells were washed with PBS and imaged for phalloidin at (ex. 540/ em. 565) using an EVOS FI fluorescent microscope (Life Technologies).

\subsubsection{Immunocytochemistry}

Cells were prepare as described above for quantitative real-time PCR. Next, cells were fixed and immunostained for $\alpha$-SMA, and Collagen- 1 as described elsewhere ${ }^{25}$.

\subsubsection{Cell proliferation assay}

hPSCs were seeded at a density of 2.500 cells/well into a 96-well plate, starved for $24 \mathrm{~h}$ and activated with $5 \mathrm{ng} / \mu \mathrm{l}$ TGF- $\beta$ or Panc- 1 conditioned medium. Cell number was monitored every $24 \mathrm{~h}$ over a time period of $72 \mathrm{~h}$. To measure the cell number, 10 $\mu$ l of Alamar Blue dye (Invitrogen, Carlsbad, USA) in $100 \mu$ of hPSC growth media was added per well. The fluorescent signal was measured after incubation for $4 \mathrm{~h}$ at $37{ }^{\circ} \mathrm{C}$ using a VIKTOR ${ }^{\mathrm{TM}}$ plate reader (Perkin Elmer, Waltham, Massachusetts).

\subsubsection{D-Collgen I Gel Contraction Assay}

A collagen suspension consisting of $3.0 \mathrm{ml}$ Collagen $\mathrm{G} 1(5 \mathrm{mg} / \mathrm{ml}$, Matrix biosciences, Morlenbach, Germany), $0.5 \mathrm{ml}$ 10x M199 medium (Sigma Aldrich), $85 \mathrm{ul} 1 \mathrm{~N} \mathrm{NaOH}$ (Sigma Aldrich) and sterile water was mixed with $1.0 \mathrm{ml}$ of cell suspension containing 200.000 shCTR or shITGA11 hPSCs. Per well of a 24 well plate $600 \mu \mathrm{l}$ of collagen gel / cell was plated and allowed to form a gel for $1 \mathrm{~h}$ at $37^{\circ} \mathrm{C}$. Once the collagen gel was formed, $1 \mathrm{ml}$ of serum free medium was added with or without TGF $\beta(5 \mathrm{ng} / \mathrm{ml})$ or Panc-1 conditioned medium (CM). Next, the gels were detached from the walls of the well plate with a $10 \mu$ pipette tip. Representative images were made at $72 \mathrm{~h}$ using a digital camera. Measurement of collagen gel diameter were normalized with the diameter of the respective well and performed using Image $\mathrm{J}$ imaging software (NIH, Bethesda, MD). 


\subsubsection{Conditioned Media}

To collect hPSC conditioned medium, shCTR and shITGA11 hPSCs were seeded into a 12 well plate at a seeding density of 40.000 cells/ well. As previously described, cells were starved and activated with TGF- $\beta$ or Panc- 1 conditioned medium for $24 \mathrm{~h}$. Next, the cells were washed 3 times with serumfree medium. After $48 \mathrm{~h}$ of incubation with serum-free medium, the medium was collected for use in conditioned medium experiments.

\subsubsection{Wound Healing Assay}

Cells were seeded into a 24 -well plate at a seeding density of 50.000 cells/well. Cells were starved and TGF- $\beta$ activated as described previously. To study migration, a scratch was made on the culture plate using a $200 \mu$ l pipette tip fixed in a custom-made holder. The cells were washed to remove all detached cells and incubated with fresh serum-free media. Images were captured at $\mathrm{t}=0 \mathrm{~h}$ and $\mathrm{t}=12 \mathrm{~h}$, with an EVOS microscope. Images were analyzed by Image $\mathbf{J}$ software to calculate the area of the scratch and represented as the percentage of wound closure.

\subsubsection{Transwell Migration Assay}

Panc- 1 cells were seeded at a density of 50.000 cells/well into the upper chamber of a 24 well $8 \mu \mathrm{m}$ transwell insert (Sigma Aldrich). The lower compartment of the transwell insert was filled with conditioned medium from shITGA11 and shCTR hPSCs as chemoattractant. After $24 \mathrm{~h}$, the cells were fixed with ice cold methanol (Fisher Scientific) for 10 min and incubated with $0.1 \%$ Crystal Violet solution (Sigma Aldrich) in 25\% methanol (Fisher Scientific) for $10 \mathrm{~min}$. After washing the cells with MilliQ water, cells from the upper compartment were removed with a cotton swap. After drying the inserts the migrated cells were imaged with a Nikon microscope (Nikon Eclipse E400, Tokyo, Japan). The positively stained area per picture was analyzed by Image $\mathbf{J}$ software.

\subsubsection{Transwell Invasion Assay}

The upper and lower compartment of $8 \mu \mathrm{m}$ BD Matrigel ${ }^{\mathrm{TM}}$ Invasion Chambers (BD Biosciences, Bedford, USA) were filled with warm DMEM containing 0\% FBS and rehydrated for $2 \mathrm{~h}$ at $37^{\circ} \mathrm{C}$. Next the DMEM was removed from both compartments and Panc- 1 cells were seeded at a density of 50.000 cells/well into the upper compartment of the Invasion Chamber. The lower compartment of the transwell insert was filled with conditioned medium from shITGA11 and shCTR hPSCs as chemoattractant. After $48 \mathrm{~h}$, the gel from the upper compartment was removed with a cotton swap. Next, the cells were fixed with ice cold methanol 
(Fisher Scientific) for $10 \mathrm{~min}$ and incubated with $0.1 \%$ Crystal Violet solution (Sigma Aldrich) in 25\% methanol (Fisher Scientific) for $10 \mathrm{~min}$ and subsequently washed with MilliQ water. After drying the inserts the invaded cells were imaged with a Nikon microscope (Nikon Eclipse E400, Tokyo, Japan). The positively stained area per picture was analyzed by Image J software.

\subsubsection{Cytokine proteome profiler protein array}

The conditioned medium to be tested in the cytokine array was collected as described previously. The RayBioß C-Series Human Cytokine Array C5 was performed as described by the manufacturer. Cytokine array membranes were imaged using a FluorChem ${ }^{\mathrm{TM}}$ M System (ProteinSimple). The protein levels were quantified by Image J Software (NIH, MD).

\subsection{Results}

\subsubsection{ITGA11 is specifically expressed on CAFs in human PDAC} Immunohistochemical analyses for ITGA11 expression in human PDAC sections showed a clear ITGA11 expression in tumor stroma while no expression in the tumor epithelial cells. In addition, ITGA11 was completely absent in normal human pancreatic tissues (Figure $1 \mathrm{~A}$ ). Visual scoring ( $\mathrm{n}=6$ patients) showed that $66.7 \%$ of stained tumors showed a moderate expression of ITGA11 in the tumor stroma while $33.3 \%$ showed a high stromal expression. Additionally, we examined the expression of the well-known CAF marker $\alpha$ SMA in combination with ITGA11 in PDAC sections (Figure $1 \mathrm{~B}$ ). Scoring of the co-localization of these two markers showed that $80 \%$ of $\alpha$-SMA positive cells were also positive for ITGA11, $20 \%$ of $\alpha$-SMA positive cells were negative for ITGA11, while only $5 \%$ of ITGA11 positive cells were negative for $\alpha$-SMA (Figure $1 \mathrm{~B}$ ).

\subsubsection{TGF- $\beta$ induces ITGA11 expression in hPSCs}

To determine whether ITGA11 expression is specifically induced in primary human activated hPSCs, we compared the gene and protein expression of ITGA11 in TGF- $\beta$-activated and non-activated control hPSCs. We found that ITGA11, on both gene and protein level, expressed only in low levels in nonactivated hPSCs, while its expression levels were induced in TGF- $\beta$-activated cells (Figure 2 A, B). Also, the induction of ITGA11 was in line with hPSC activation and the CAF markers, collagen-I (Col1a1) and $\alpha$-SMA, (Figure $2 \mathrm{C}$, 
D). Additionally, no expression of ITGA11 was found in the human pancreatic cancer cell lines Panc-1, MiaPaCa-2 and AsPc-1 (Figure 2 B).

A
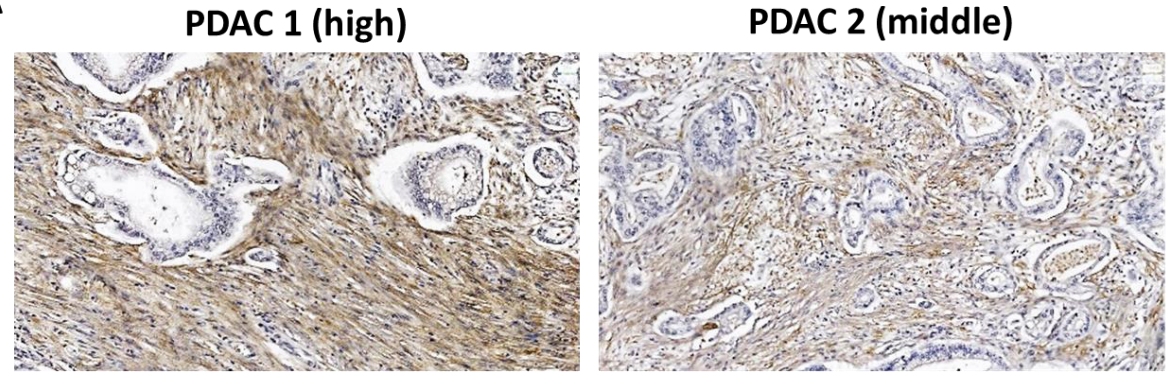

PDAC 3 (middle)

\section{Normal Pancreas}
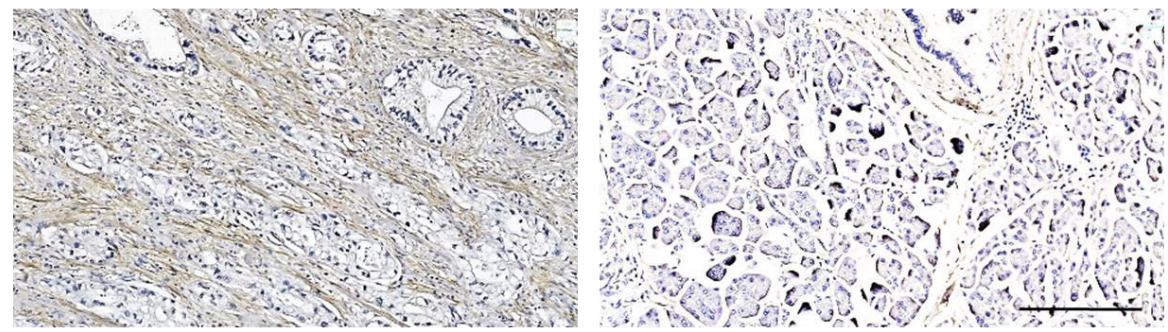

B

ITGA11

a-SMA
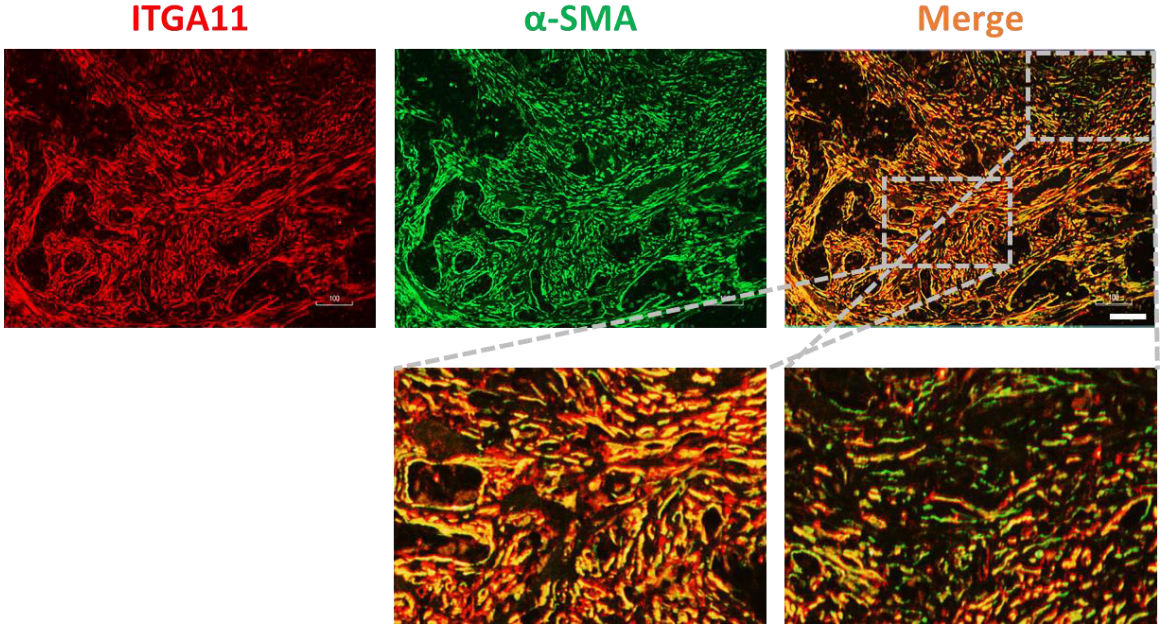

Figure 1. ITGA11 is specifically expressed on CAFs in human PDAC. A) Staining of human PDAC and normal pancreas for ITGA11. Scale bar $=250 \mu \mathrm{m}$. B) Co-localization of ITGA11 with $\alpha$-SMA. Scale bar $=100 \mu \mathrm{m}$. 
A

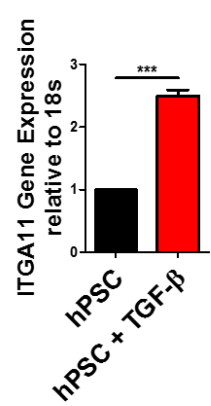

B

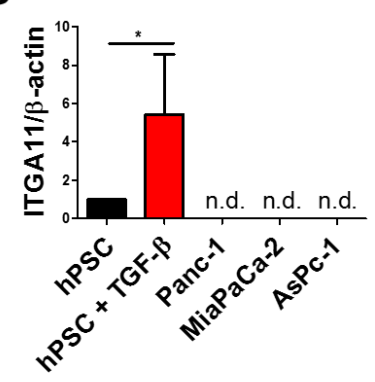

ITGA11

$\beta$-actin

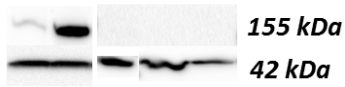

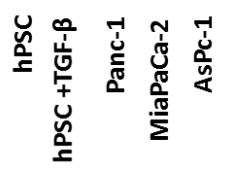

C

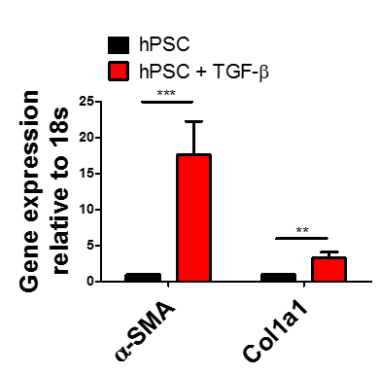

D

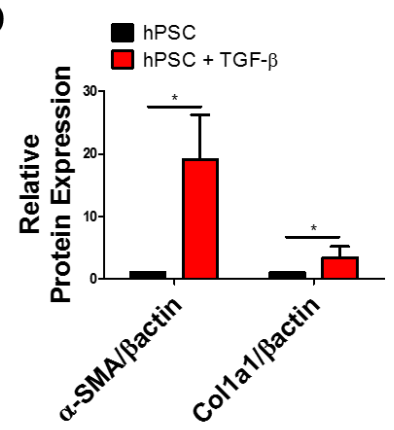

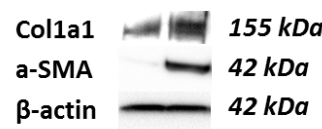

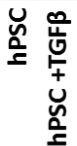

Figure 2. TGF- $\beta$ induces ITGA11 expression in hPSCs. A) Gene expression and B) protein expression of ITGA11 in TGF- $\beta$-activated and non-activated hPSCs. C) Gene expression and D) protein expression of $\alpha$-SMA and Collal in TGF- $\beta$-activated and nonactivated hPSCs Data represent mean + SEM for at least 3 independent experiments. Statistical analysis was performed by two-tailed unpaired student $t$-test. $*_{p}<0.05$, **p $<0.01, * * * p<0.001 . n . d$. stands for not detected.

\subsubsection{Knockdown of ITGA11 inhibits TGF- $\beta$ mediated differentiation of hPSCs}

To demonstrate the role for ITGA11 in hPSC activation, we stably knocked down ITGA11 in hPSCs using shRNA plasmid and evaluated the effect of the knockdown on the hPSC phenotype and TGF- $\beta$-mediated activation and differentiation. The knockdown of ITGA11 was confirmed using real time qPCR and western blot analysis, showing a reduction of about $50 \%$ at the mRNA level and about $85 \%$ at the protein level in ITGA11 knockdown (shITGA11) hPSC compared to the control (shCTR) hPSCs (Figure 3 A, B). ITGA11 knockdown resulted in a drastic phenotypic change leading to cellular hypertrophy compared to shCTR hPSCs (Figure $3 \mathrm{C}$ ). Upon activation with TGF- $\beta$, both shCTR and shITGA11 hPSCs showed only a slight induction in ITGA11 levels (Figure 3 D). Surprisingly, the induction of ITGA11 and $\alpha$-SMA protein levels with TGF- $\beta$ 
was less in shCTR hPSCs when compared with normal hPSCs (Figure 2B), which can be attributed to higher passage numbers which was unavoidable to achieve stable transfection. Strikingly, TGF- $\beta$-mediated induction of $\alpha$-SMA in shITGA11 hPSCs was significantly attenuated compared to shCTR hPSCs (Figure 3D). Immunostaining showed the same trend for the protein expression of Col1a1 and $\alpha$-SMA (Figure 3E).

Additionally, we examined the effect of ITGA11 knockdown on CAF marker and found that ITGA11 knockdown significantly downregulated the gene expression of CAF markers such as Platelet derived growth factor $\beta$ receptor (PDGF $\beta R$ ), Desmin, Collagen 3 (Col3a1), NAD(P) Dependent Steroid Dehydrogenase-Like (NSDHL), Insulin-induced gene 1 (INSIG1), Isopentenyldiphosphate Delta-isomerase 1 (IDI1) and C-X-C chemokine receptor type 4 (CXCR-4) (Figure 3F). Upon activation with TGF- $\beta$, shITGA11 hPSCs showed an attenuated expression in the CAF markers PDGF $\beta$ R, Desmin, NSDHL, INSIG1, IDI1, MMP-2 and CXCR-4 as compared to shCTR hPSCs (Figure 3E), indicating that these genes are regulated by ITGA11. We further analyzed shCTR and shITGA11 hPSCs with and without TGF- $\beta$-mediated activation for the mRNA gene expression profiling of $84 \mathrm{ECM}$ and adhesion proteins using a human profiler gene array. Genes downregulated after the knockdown of ITGA11 included various receptors responsible for the adhesion to immune cells (SELE, ITGB2, SELP, ITGAL, SELL, ITGAM), laminin receptors (ITGA7, ITGB4), collagen receptors (ITGA8), ECM molecules (COL11A1, ECM1, LAMA1, VTN), MMPs and TIMPs (MMP8, MMP9, MMP10, MMP11, MMP12, MMP13 and TIMP3), cell adhesion molecules (CNTN1, CDH1, CTNND2, THBS2, CLEC3B, NCAM1) and a protein involved in ECM production (HAS1) (Table 4). Furthermore, we identified TGF- $\beta$-induced genes which are regulated by ITGA11. We found several genes whose expression was not induced in shITGA11 hPSC but in shCTR hPSC after activation with TGF$\beta$ (Table 5). These included cell adhesion molecules (THBS1, SPP1), growth factors (CTGF), ECM molecules (TNC, FN1, COL8A1, TGFBI, COL5A1), MMPs and TIMPs (TIMP1, MMP3) and protein involved in ECM production (SPARC). 

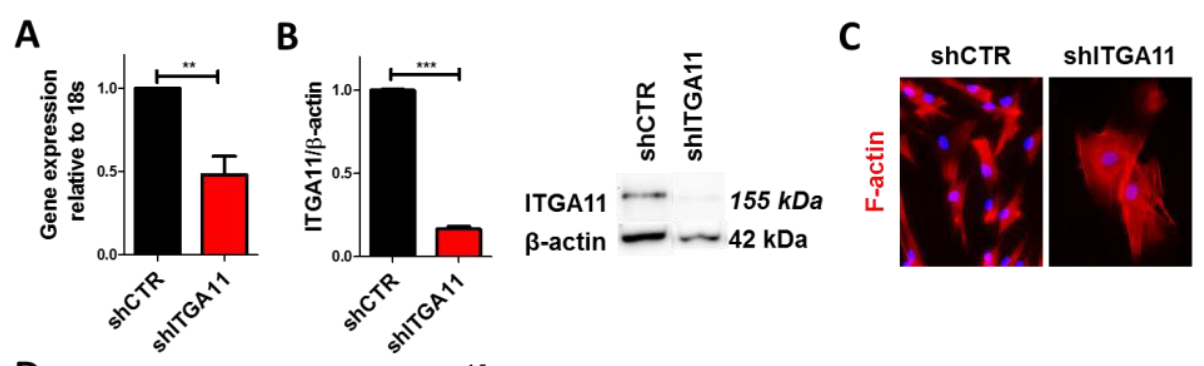

D
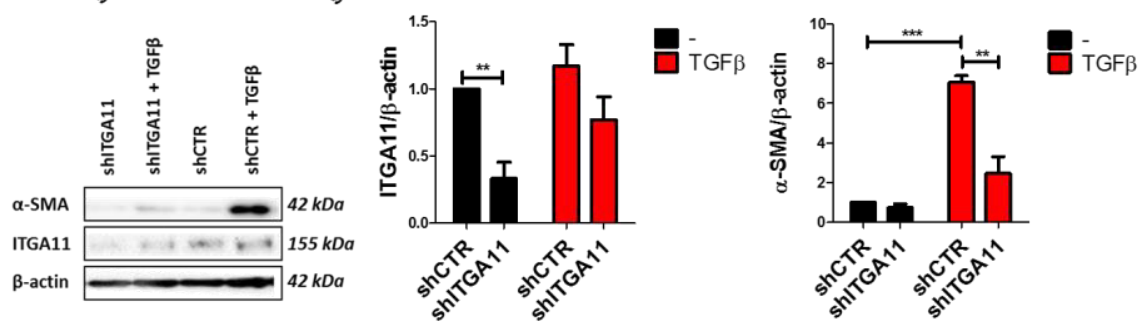

E

ShCTR

ShITGA11

ShCTR + TGF- $\beta$

shITGA11 + TGF- $\beta$

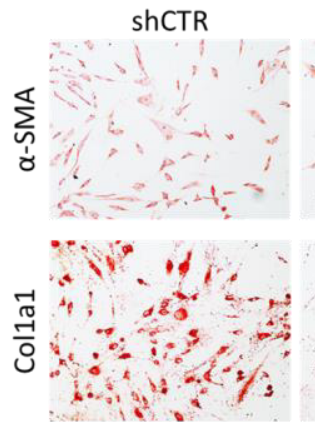

F
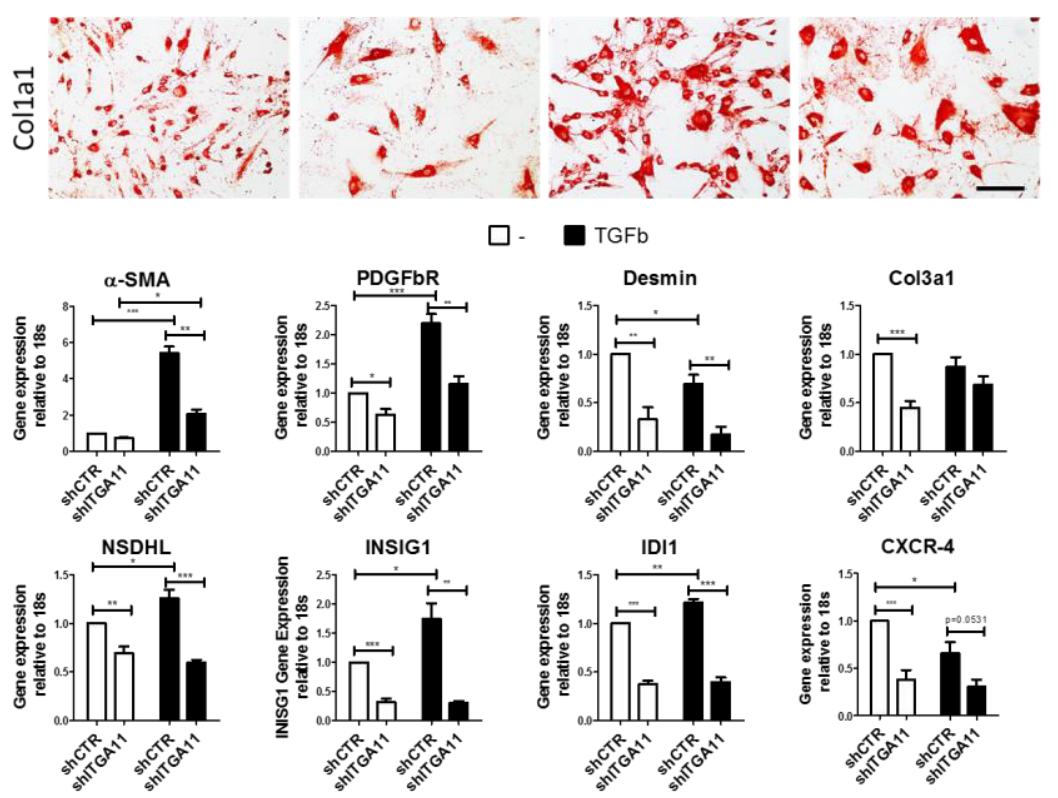

Figure 3. Knockdown of ITGA11 inhibits TGF-ß mediated differentiation of hPSCs. A) Real time qPCR and B) Western Blot the shRNA mediated knockdown of ITGA11. C) F-actin staining of shCTR compared to shITGA11 hPSCs. Scale bar = $100 \mu \mathrm{m} . \mathrm{D})$ Western blot for ITGA11 and $\alpha$-SMA of shCTR and shITGA11 hPSCs with and without 
TGF- $\beta$ activation. E) Immunostaining for $\alpha$-SMA and Collal of shCTR and shITGA11 hPSCs with and without TGF- $\beta$ activation. Scale bar $=200 \mu m . F)$ Real time qPCR for the CAF markers $\alpha$-SMA, PDGF $\beta R$, Desmin, Col3a1, NSDHL, INSIG1, IDI1 and CXCR4. Data represent mean + SEM for at least 3 independent experiments. Statistical analysis was performed by two-tailed unpaired student t-test. ${ }^{*} p<0.05$, **p $<0.01$, $* * * p<0.001$.

Table 4. Genes downregulated in shITGA11 hPSCs compared with shCTR hPSCs with a fold regulation $\leq-2$. The data was obtained with the $R T^{2}$ Profiler ${ }^{\mathrm{TM}}$ PCR Array Human Extracellular Matrix \& Adhesion Molecules (Qiagen) and analyzed using the data analysis center provided by Qiagen.

\begin{tabular}{|c|c|c|c|}
\hline Gene ID & Refseq & Description & Fold Regulation \\
\hline SELE & NM_000450 & Selectin E & $-11,26$ \\
\hline CNTN1 & NM_001843 & Contactin 1 & $-9,31$ \\
\hline TIMP3 & NM_000362 & TIMP metallopeptidase inhibitor 3 & $-7,25$ \\
\hline $\mathrm{CDH} 1$ & NM_004360 & Cadherin 1 & $-5,93$ \\
\hline ITGA7 & NM_001144996 & Integrin subunit alpha 7 & $-5,57$ \\
\hline MMP8 & NM_002424 & Matrix metallopeptidase 8 & $-4,89$ \\
\hline CTNND2 & NM_001332 & Catenin delta 2 & $-4,59$ \\
\hline ITGB4 & NM_000213 & Integrin subunit beta 4 & $-4,57$ \\
\hline HAS1 & NM_001523 & Hyaluronan synthase 1 & $-4,49$ \\
\hline ITGB2 & NM_000211 & Integrin subunit beta 2 & $-4,42$ \\
\hline THBS2 & NM_003247 & Thrombospondin 2 & $-3,68$ \\
\hline COL11A1 & NM_001854 & Collagen type XI alpha 1 chain & $-3,38$ \\
\hline SELP & NM_003005 & Selectin P & $-3,33$ \\
\hline ECM1 & NM_004425 & Extracellular matrix protein 1 & $-3,04$ \\
\hline ITGAL & NM_002209 & Integrin subunit alpha L & $-2,96$ \\
\hline MMP10 & NM_002425 & Matrix metallopeptidase 10 & $-2,67$ \\
\hline MMP11 & NM_005940 & Matrix metallopeptidase 11 & $-2,65$ \\
\hline SELL & NM_000655 & Selectin L & $-2,63$ \\
\hline MMP9 & NM_004994 & Matrix metallopeptidase 9 & $-2,57$ \\
\hline CLEC3B & NM_003278 & C-type lectin domain family 3 member B & $-2,52$ \\
\hline LAMA1 & NM_005559 & Laminin subunit alpha 1 & $-2,51$ \\
\hline ITGAM & NM_001145808 & Integrin subunit alpha $\mathrm{M}$ & $-2,45$ \\
\hline MMP12 & NM_002426 & Matrix metallopeptidase 12 & $-2,44$ \\
\hline ITGA8 & NM_003638 & Integrin subunit alpha 8 & $-2,41$ \\
\hline VTN & NM_000638 & Vitronectin & $-2,34$ \\
\hline MMP13 & NM_002427 & Matrix metallopeptidase 13 & $-2,21$ \\
\hline
\end{tabular}


Table 5. Genes upregulated in TGFß-activated shCTR and shITGA11 hPSCs compared with their respective control. The data was obtained with the $R T^{2}$ Profiler ${ }^{\mathrm{TM}}$ PCR Array Human Extracellular Matrix \& Adhesion Molecules (Qiagen) and analyzed using the data analysis center provided by Qiagen.

\begin{tabular}{|c|c|c|c|c|c|}
\hline \multirow{3}{*}{\begin{tabular}{|l} 
Gene ID \\
THBS1
\end{tabular}} & \multirow{3}{*}{$\begin{array}{l}\text { Refseq } \\
\text { NM_003246 }\end{array}$} & \multirow{3}{*}{$\begin{array}{l}\text { Description } \\
\text { Thrombospondin } 1\end{array}$} & \multirow{2}{*}{\multicolumn{2}{|c|}{ 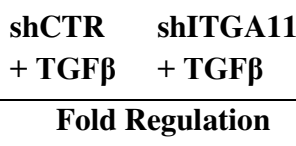 }} & \multirow{3}{*}{$\begin{array}{l}\Delta \text { Difference } \\
10,94\end{array}$} \\
\hline & & & & & \\
\hline & & & 14,26 & 3,32 & \\
\hline CTGF & NM_001901 & $\begin{array}{l}\text { Connective tissue } \\
\text { growth factor }\end{array}$ & 6,22 & 2,81 & 3,41 \\
\hline TNC & NM_002160 & Tenascin C & 7,65 & 5,92 & 1,73 \\
\hline TIMP1 & NM_003254 & $\begin{array}{l}\text { TIMP } \\
\text { metallopeptidase } \\
\text { inhibitor } 1\end{array}$ & 2,49 & 1,06 & 1,43 \\
\hline MMP3 & NM_002422 & $\begin{array}{l}\text { Matrix } \\
\text { metallopeptidase } 3\end{array}$ & 2,41 & 1,43 & 0,98 \\
\hline SPP1 & $\begin{array}{l}\text { NM_0012518 } \\
30\end{array}$ & $\begin{array}{l}\text { Secreted } \\
\text { phosphoprotein } 1\end{array}$ & 2,71 & 1,82 & 0,89 \\
\hline FN1 & NM_212474 & Fibronectin 1 & 2,28 & 1,73 & 0,55 \\
\hline $\begin{array}{l}\text { COL8A } \\
1\end{array}$ & NM_001850 & $\begin{array}{l}\text { Collagen type VIII } \\
\text { alpha } 1 \text { chain }\end{array}$ & 2,47 & 1,96 & 0,51 \\
\hline SPARC & NM_003118 & $\begin{array}{l}\text { Secreted protein } \\
\text { acidic and cysteine } \\
\text { rich }\end{array}$ & 2,16 & 1,86 & 0,3 \\
\hline TGFBI & NM_000358 & $\begin{array}{l}\text { Transforming } \\
\text { growth factor beta } \\
\text { induced }\end{array}$ & 2,25 & 1,96 & 0,29 \\
\hline
\end{tabular}

\subsubsection{Knockdown of ITGA11 inhibits Panc-1 conditioned medium mediated differentiation of hPSCs}

We investigated the influence of Panc-1 conditioned medium on ITGA11 expression and Panc-1 conditioned medium induced activation of hPSCs and shITGA11 hPSCs. Unlike TGF- $\beta$ treatment, treatment of hPSCs with Panc-1 CM did not induce ITGA11 levels in either shITGA11 or shCTR hPSCs (Figure 4 A). The protein expression of $\alpha$-SMA was induced in shCTR hPSCs activated with Panc-1 CM, while these inductions were attenuated in shITGA11 hPSCs (Figure $4 \mathrm{~A}$ ). Immunostaining showed the same trend for the protein expression of Colla1 and $\alpha$-SMA (Figure 4 B). 
A
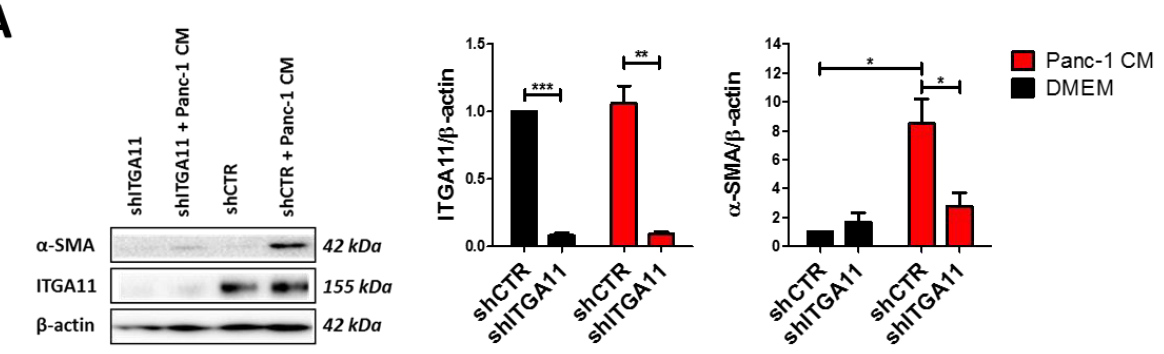

B

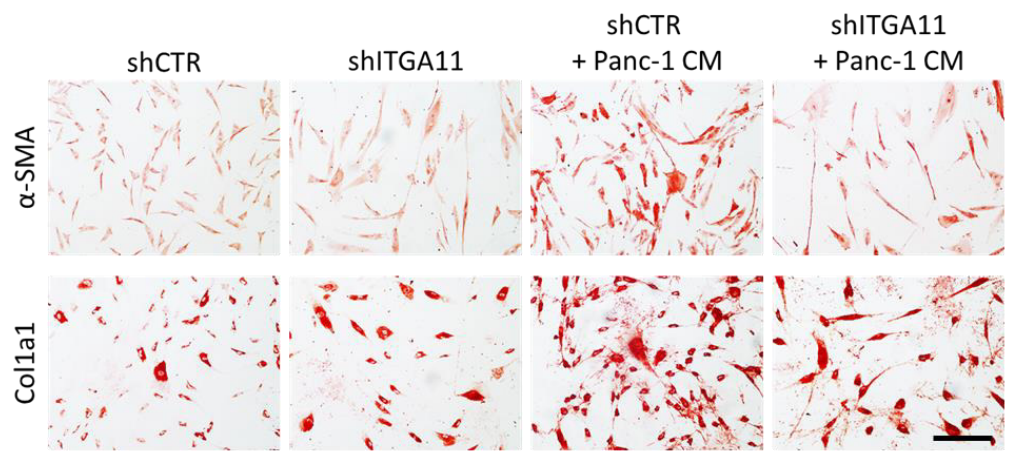

C

$\square$ Medium $\square$ Panc-1 CM

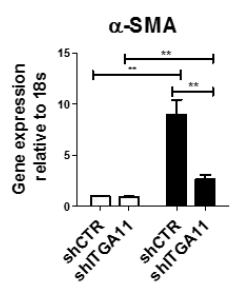

NSDHL

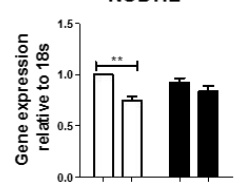

की

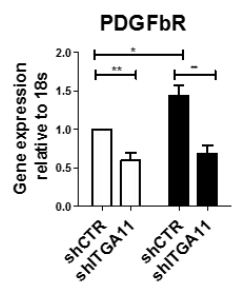

INSIG-1
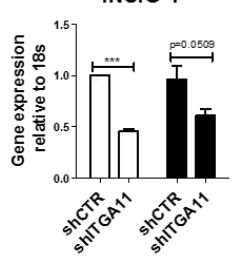

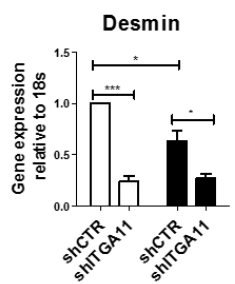

IDI1

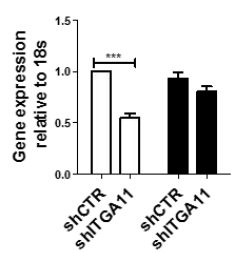

Col3a1

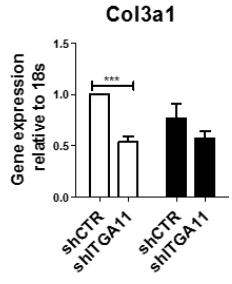

CXCR-4

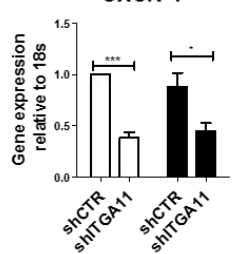

Figure 4. Knockdown of ITGA11 inhibits Panc-1 conditioned medium mediated differentiation of hPSCs. A) Western blot for ITGA11 and $\alpha$-SMA of shCTR and shITGA11 hPSCs with and without Panc-1 CM activation. B) Immunostaining for $\alpha$ SMA and Collal of shCTR and shITGAl1 hPSCs with and without Panc-1 CM activation. Scale bar $=200 \mu \mathrm{m} . C$ ) Real time $q P C R$ of the CAF markers $\alpha-S M A$, PDGF $\beta R$, Desmin, Col3a1, NSDHL, INSIG-1, IDII and CXCR-4. Data represent mean + SEM for at least 3 independent experiments. Statistical analysis was performed by two-tailed unpaired student t-test. $* p<0.05$, **p $<0.01$, ***p $<0.001$. 
Analysis of the gene expression of well-known CAF markers in shITGA11 revealed significant downregulation of PDGF $\beta$, Desmin, Col3a1, NSDHL, INSIG-1, IDI-1 and CXCR-4 as compared to shCTR hPSCs (Figure 4 C). When these cells were activated with Panc-1 CM, the CAF markers $\alpha$-SMA, PDGF $\beta$ R, Desmin, INSIG-1 and CXCR-4 showed an attenuated expression in shITGA11 as compared to shCTR hPSCs (Figure $4 \mathrm{C}$ ). It needs to be noted, that among these markers only PDGF $\beta R$ was significantly induced with Panc-1 CM (Figure $4 \mathrm{C})$.

\subsubsection{Knockdown of ITGA11 attenuates TGF- $\beta$ induced hPSC function}

We furthermore investigated whether ITGA11 regulates the functions of hPSCs including migration and contraction. Human PSCs are known for their ability to migrate to the tumor site and differentiate into CAFs with high contractile properties and form metastasis ${ }^{26}$.
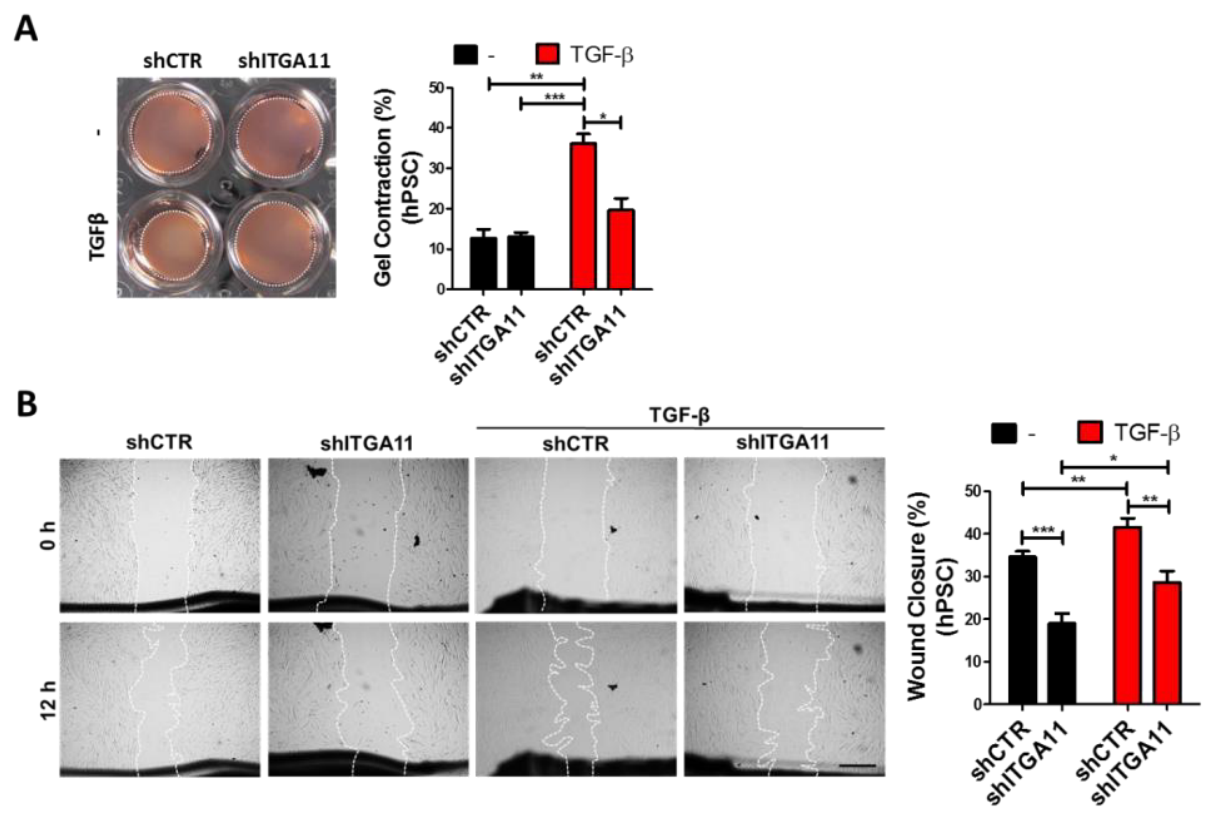

Figure 5. Knockdown of ITGA11 attenuates TGF- $\beta$ induced hPSC function. A) Representative images and quantification of collagen gel contraction of shCTR and shITGA11 hPSCs with and without TGF- $\beta$ activation. B) Representative microscopic images and quantification showing the migration of shCTR and shITGAll hPSCs with and without TGF- $\beta$ activation. Scale bar $=500 \mu \mathrm{m}$. Data represent mean + SEM for at least 3 independent experiments. Statistical analysis was performed by two-tailed unpaired student t-test. $* p<0.05, * * p<0.01$, ***p $<0.001$. 
While non-activated hPSCs do not have the ability to contract, hPSCs activated with TGF- $\beta$ acquire a contractile phenotype. In comparison to shCTR hPSCs, shITGA11 hPSCs showed significantly poor contractile ability (Figure 5 A). Furthermore, cell migration (with and without TGF $\beta$ induction) was attenuated in shITGA11 hPSCs as compared to shCTR hPSCs (Figure $5 \mathrm{~B}$ ).

\subsubsection{Knockdown of ITGA11 attenuates Panc-1 CM induced hPSC function}

Next to TGF- $\beta$, we also investigated the effect of ITGA11 knockdown in hPSCs on functional activation with Panc-1 CM. Similar to TGF- $\beta$ activation, the activation with Panc-1 CM led to the induction of the contractility of shCTR hPSCs and these inductions were diminished in shITGA11 hPSCs (Figure 6 A). In contrast to TGF $\beta$ activation, Panc- $1 \mathrm{CM}$ did not show induction of migration of shCTR hPSC but yet ITGA11 knockdown reduced the migration of hPSCs (Figure $6 \mathrm{~B}$ ). These data indicate that ITGA11 is able to regulate the activation of hPSCs by controlling more pathways than only TGF- $\beta$.
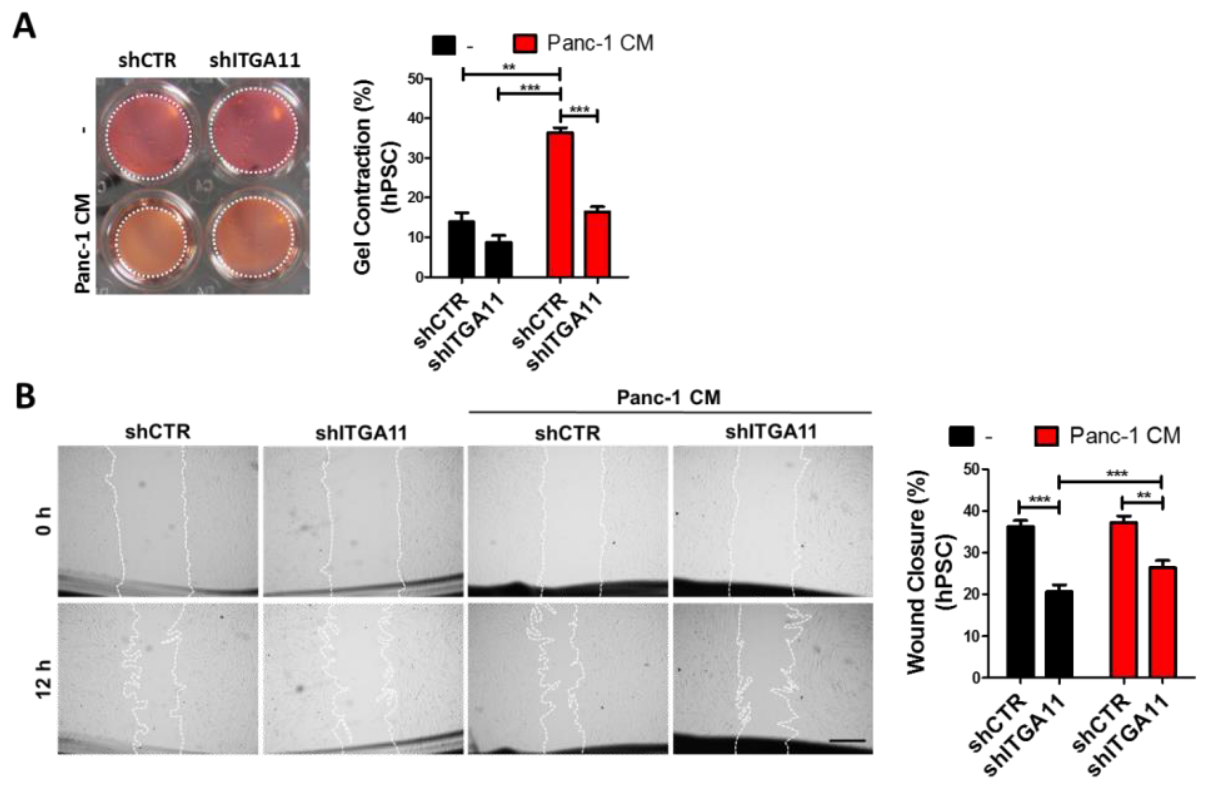

Figure 6. Knockdown of ITGA11 attenuates Panc-1 CM induced hPSC function. A) Representative images and quantification of collagen gel contraction by shCTR and shITGA11 hPSCs with and without Panc-1 CM activation. B) Representative microscopic images and quantification showing the migration of shCTR and shITGA11 hPSCs with and without Panc-1 CM activation. Scale bar $=500 \mu \mathrm{m}$. Data represent 
mean + SEM for at least 3 independent experiments. Statistical analysis was performed by two-tailed unpaired student $t$-test. $* * p<0.01$, ***p $<0.001$.

\subsubsection{Effect of ITGA11 on the secretome of hPSCs}

Furthermore, we analyzed the effect of ITGA11 knockdown on the secretome of hPSCs, which is crucial for the crosstalk with tumor cells and other cells in the tumor microenvironment. We found a decrease or increase in the secretion of several cytokines and growth factors by shITGA11 hPSCs compared to shCTR hPSCs (Supplementary Table 1, Figure 7).

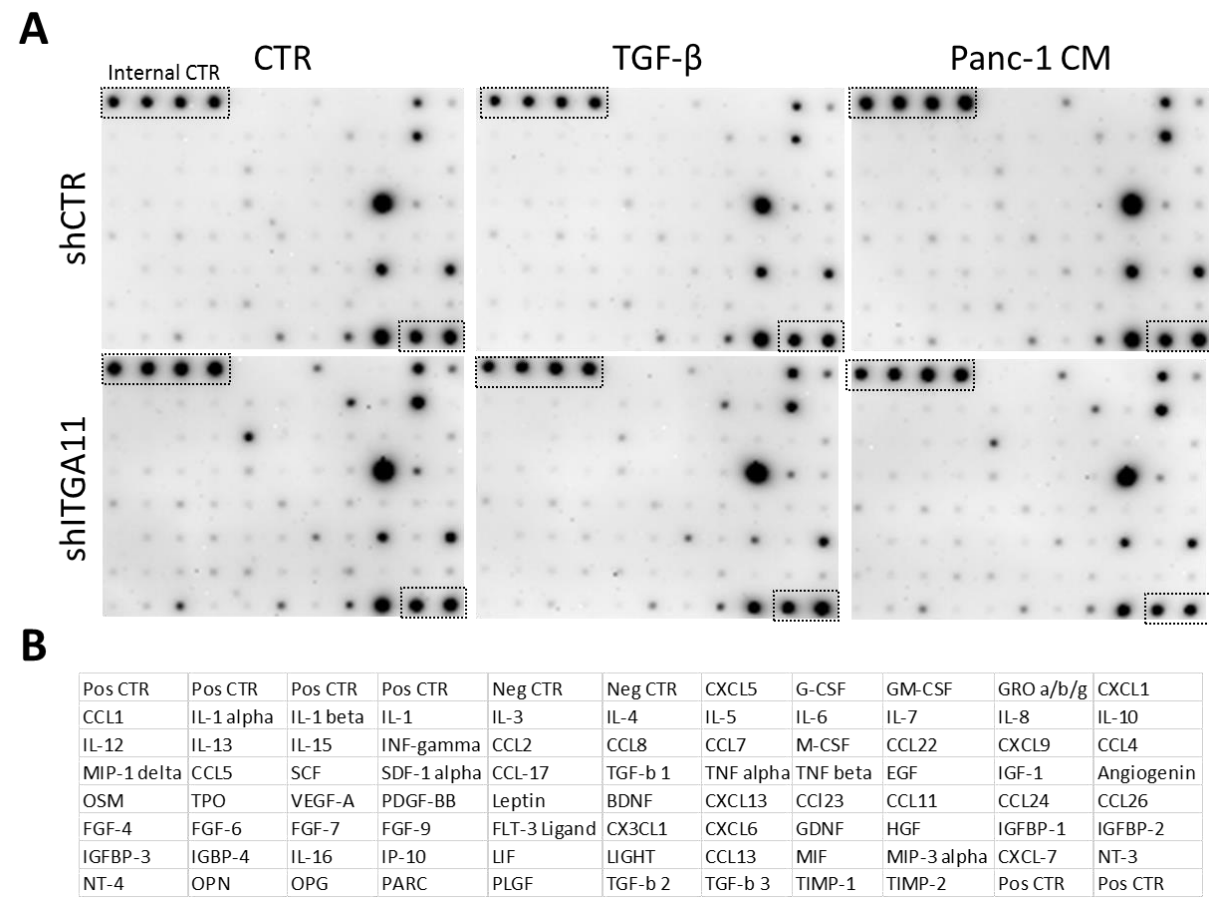

Figure 7. Effect of ITGA11 on the secretome of hPSCs. A) Results from the Human Cytokine Antibody Array C5 (RayBioß) for shCTR and shITGA11 hPSC, without and with activation with TGF- $\beta$ or Panc-1 CM. Boxes indicate internal positive controls. B) Map pf the Human Cytokine Antibody Array C5.

The top five downregulated and upregulated cytokines and growth factors are shown in Table 6. Additionally, we found that the growth factors MIF, TGF- $\beta 3$, IGFBP-1, IGFBP-3, CXCL1, PDGF-BB, FGF-6, FGF-7, CCL22 and CCL23 were induced by TGF- $\beta$ in shCTR hPSCs, while their expression was attenuated in shITGA11 hPSCs treated with TGF- $\beta$ (Table 7). On the contrary, we found 
LIF, CCL4 and M-CSF to be induced to a higher extent in shITGA11 compared to shCTR hPSCs upon treatment with TGF- $\beta$ (Table 7, Figure 7).

Table 6. Top 10 cytokines and growth factors downregulated or upregulated in shITGA11 hPSCs compared with shCTR. The data was obtained with the Human Cytokine Antibody Array C5 (RayBio $\left.{ }^{\circledR}\right)$ and quantified using ImageJ. Blot of the Human Cytokine Antibody Array C5 can be found in figure 7.

\begin{tabular}{lll}
\hline Protein ID & Description & Fold Regulation \\
\hline M-CSF & Macrophage colony-stimulating factor & $-2,87$ \\
Angiogenin & Angiogenin & $-2,09$ \\
CCL4 & C-C motif chemokine 4 & $-1,89$ \\
TGF-b 3 & Transforming growth factor beta 3 & $-1,83$ \\
CCL22 & C-C motif chemokine 22 & $-1,83$ \\
FGF-6 & Fibroblast growth factor 6 & 1,89 \\
CCL2 & C-C motif chemokine 2 & 2,06 \\
MIF & Macrophage migration inhibitory factor & 2,15 \\
OPG & Osteoprotegerin & 2,49 \\
IL-6 & Interleukin-6 & 3,06
\end{tabular}

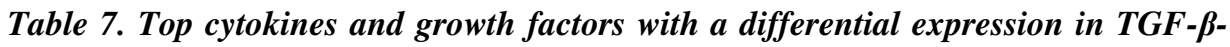
activated shCTR and shITGA11 hPSCs. Only cytokines and growth factors which showed $\geq 1.1$ fold-upregulation with Panc-1 CM in shCTR hPSCs and a $\triangle$ difference $\geq$ 0.1 are shown. The data was obtained with the Human Cytokine Antibody Array C5 (RayBio $\left.{ }^{\circledR}\right)$ and analyzed using ImageJ. Blot of the Human Cytokine Antibody Array C5 can be found in figure 7 .

\begin{tabular}{|c|c|c|c|c|}
\hline \multirow{3}{*}{$\begin{array}{l}\text { Protein ID } \\
\text { MIF }\end{array}$} & \multirow{3}{*}{$\begin{array}{l}\text { Description } \\
\text { Macrophage migration inhibitory } \\
\text { factor }\end{array}$} & $\begin{array}{l}\text { shCTR } \\
+ \text { TGF- } \beta\end{array}$ & \multicolumn{2}{|c|}{$\begin{array}{l}\text { shITGA11 } \\
+ \text { TGF- } \beta\end{array}$} \\
\hline & & \multicolumn{2}{|c|}{ Fold Regulation } & $\Delta$ Difference \\
\hline & & 1,24 & $-1,20$ & 2,44 \\
\hline TGF-b 3 & Transforming growth factor beta 3 & 1,19 & $-1,18$ & 2,37 \\
\hline IGFBP-1 & $\begin{array}{l}\text { Insulin-like growth factor-binding } \\
\text { protein } 1\end{array}$ & 1,32 & $-0,84$ & 2,16 \\
\hline CXCL1 & Growth-regulated alpha protein & 1,20 & $-0,88$ & 2,07 \\
\hline PDGF-BB & $\begin{array}{l}\text { Platelet-derived growth factor } \\
\text { subunit B }\end{array}$ & 1,69 & $-0,38$ & 2,07 \\
\hline IGFBP-3 & $\begin{array}{l}\text { Insulin-like growth factor-binding } \\
\text { protein } 3\end{array}$ & 1,71 & $-0,24$ & 1,95 \\
\hline FGF-6 & Fibroblast growth factor 6 & 1,58 & $-0,32$ & 1,90 \\
\hline
\end{tabular}




$\begin{array}{lllll}\text { CC123 } & \text { C-C motif chemokine 23 } & 1,23 & -0,60 & 1,83 \\ \text { FGF-7 } & \text { Fibroblast growth factor 7 } & 1,45 & 1,19 & 0,26 \\ \text { CCL22 } & \text { C-C motif chemokine 22 } & 4,33 & 4,13 & 0,20 \\ \text { LIF } & \text { Leukemia inhibitory factor } & 1,25 & 1,67 & -0,41 \\ \text { CCL4 } & \text { C-C motif chemokine 4 } & 1,83 & 2,61 & -0,79 \\ \text { M-CSF } & \text { Macrophage colony-stimulating } & 2,05 & 3,12 & -1,07 \\ & \text { factor } & & & \end{array}$

As shown previously by others, conditioned medium (CM) of Panc-1 tumor cells contains a mixture of different cytokines, like IL-8, IGFBP-1, OPG, VEGF, Angiogenin, IGFBP-2, PIGF and GM-CSF 27. We evaluated whether knockdown of ITGA1 1 in hPSCs not only attenuated TGF- $\beta$-induced secretions, but also Panc-1 condition medium (CM)-induced secretion from hPSCs. We found that the secreted factors CXCL1, IGFBP-1 and PDGF-BB were induced by Panc-1 CM in shCTR hPSCs, while their expression was attenuated in shITGA11 hPSCs treated with Panc-1 CM (Table 8, Figure 7). On the contrary, we found IGFBP-3 and CCL-2 to be more induced in shITGA11 compared to shCTR hPSCs upon treatment with Panc-1 CM (Table 8, Figure 7).

Table 8. Differential expression of Panc-1 CM-induced cytokines and growth factors in shCTR and shITGA11 hPSCs. Only cytokines and growth factors which showed $\geq$ 1.1 fold-upregulation with Panc-1 CM in shCTR hPSCs and a $\Delta$ difference $\geq 0.1$ are shown. The data was obtained with the Human Cytokine Antibody Array C5 (RayBioß) and analyzed using ImageJ. Blot of the Human Cytokine Antibody Array C5 can be found in figure 7.

\begin{tabular}{lllll} 
& \multicolumn{2}{c}{$\begin{array}{l}\text { shCTR } \\
+ \text { Panc-1 CM }\end{array}$} & $\begin{array}{l}\text { shITGA11 } \\
+ \text { Panc-1 CM }\end{array}$ & \\
\hline $\begin{array}{l}\text { Protein } \\
\text { ID }\end{array}$ & Description & Fold Regulation & $\Delta$ Difference \\
\hline CXCL1 & $\begin{array}{l}\text { Growth-regulated alpha } \\
\text { protein }\end{array}$ & 1,10 & $-1,07$ & 2,17 \\
IGFBP-1 & $\begin{array}{l}\text { Insulin-like growth factor- } \\
\text { binding protein 1 }\end{array}$ & 1,44 & $-0,62$ & 2,07 \\
PDGF- & $\begin{array}{l}\text { Platelet-derived growth } \\
\text { factor subunit B }\end{array}$ & 1,14 & $-0,36$ & 1,51 \\
BB & $\begin{array}{l}\text { Insulin-like growth factor- } \\
\text { IGFBP-3 }\end{array}$ & 1,18 & 1,61 & $-0,43$ \\
Cinding protein 3 & C-C motif chemokine 22 & 1,47 & 2,74 & $-1,27$
\end{tabular}




\subsubsection{Conditioned medium from hPSCs with knockdown of ITGA11 inhibits paracrine effects on Panc-1}

After confirming the direct effects of ITGA11 knockdown on hPSC activation, we investigated whether ITGA11 knockdown is able to inhibit hPSC-mediated paracrine effects on tumor cell migration and invasion.

A
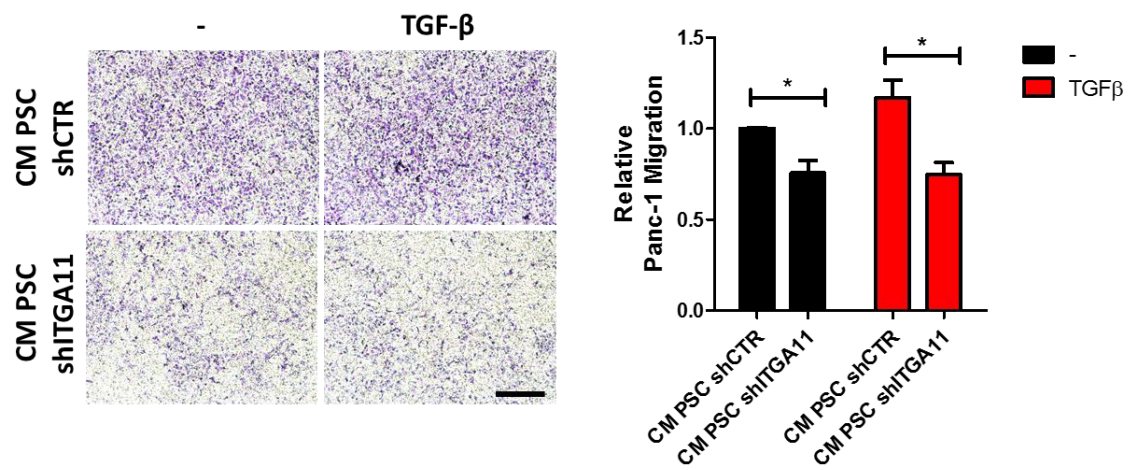

B

TGF- $\boldsymbol{\beta}$
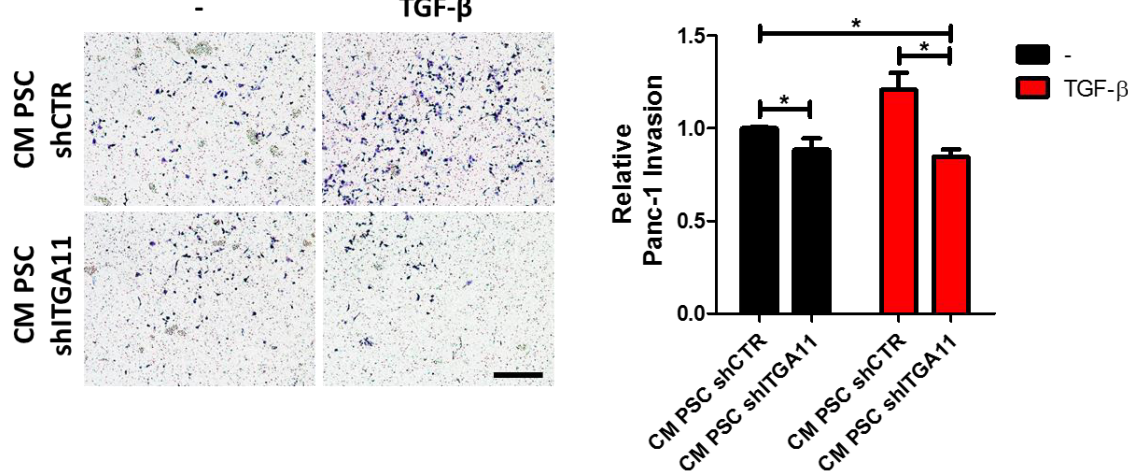

Figure 8. Conditioned medium from hPSCs with knockdown of ITGA11 inhibit paracrine effects on Panc-1. A) Representative images and quantification of Panc-1 tumor cell migration after 24 hours of incubation with conditioned medium obtained from shCTR and shITGA11 hPSCs with and without TGF- $\beta$ activation. Scale bar $=400$ $\mu m$. B) Representative images and quantification of Panc-1 tumor cell invasion after 24 hours of incubation with condition medium collected from shCTR and shITGA11 hPSCs with and without TGF- $\beta$ activation. Scale bar $=400 \mu \mathrm{m}$. Data represent mean + SEM for at least 3 independent experiments. Statistical analysis was performed by two-tailed unpaired student $t$-test. ${ }^{*} p<0.05$.

To evaluate this, we collected conditioned medium from non-activated and TGF$\beta$ activated shCTR and shITGA11 hPSCs. We found that Panc-1 cells incubated 
with CM from shITGA11 hPSCs had a reduced ability to migrate (Figure $8 \mathrm{~A}$ ) and invade (Figure $8 \mathrm{~B}$ ) in transwell inserts.

\subsection{Discussion}

The present study introduces the collagen 1 binding transmembrane receptor ITGA11 as a significant regulator of the CAF phenotypic differentiation and function. ITGA11 was shown to be substantially expressed in the stroma of human PDAC and co-localized with the major CAF marker $\alpha$-SMA in human patient samples. Strikingly, the knockdown of ITGA11 in hPSCs inhibited their differentiation, migration and contractility. A profiler array showed that many ECM and adhesion molecules were downregulated in hPSCs after ITGA11 knockdown. Moreover, our data revealed that the knockdown of ITGA11 attenuated hPSCs mediated paracrine effects on Panc-1 tumor cells.

The pancreatic tumor stroma is well known as one of the hallmarks of PDAC. CAFs, the most abundant stromal cells, promote PDAC progression by producing high amounts of ECM and secreting various cytokines and growth factors, which in turn stimulate tumor growth, angiogenesis, invasion, and metastases ${ }^{5-7,27}$. Human PSCs are regarded as the main precursors of CAFs in the pancreatic tumor stroma ${ }^{16}$ and in response to TGF- $\beta$ they acquire a CAFlike myofibroblast phenotype, as we and others have shown previously ${ }^{28-30}$. Therefore, modulating the tumor stroma through therapeutic strategies which aim to inhibit the activation of hPSCs into CAFs is a rational approach to gain therapeutic benefits for the treatment of PDAC. ECM transmembrane receptors of the integrin family, allow cells to attach, migrate and proliferate. Some integrin receptors are strongly upregulated in CAFs, which by themselves produce high amounts of ECM, including collagens ${ }^{18}$. Integrin targeting in myofibroblasts has been shown to inhibit fibrosis in multiple organs such as liver, lung and kidney ${ }^{18,24,31,32}$.

In the present study, we show that ITGA11 is highly upregulated in the tumor stroma of human PDAC. Co-immunostaining of ITGA11 with $\alpha$-SMA showed that ITGA11 was almost exclusively expressed on CAFs within these tissues, with $80 \%$ of $\alpha$-SMA positive cells being ITGA11 positive and only $20 \%$ of $\alpha$ SMA positive cells being ITGA11 negative. Interestingly, no expression of ITGA11 was found in healthy pancreatic tissue, suggesting that therapeutic targeting of this receptor is likely to have a minimal side effects on normal tissues. 
We found that quiescent hPSCs had an extremely low expression of ITGA11, while activation with TGF- $\beta$ resulted in a significant upregulation of ITGA11, which correlated with the significant upregulation of the main CAF markers $\alpha$ SMA and Colla1. The induction of ITGA11 can be attributed to the TGF- $\beta$ mediated regulation of ITGA11 in a Smad Sp-1 dependent manner which was previously demonstrated ${ }^{33}$. Stable knockdown of ITGA11 in hPSCs significantly attenuated the TGF- $\beta$ and Panc-1 CM mediated differentiation of hPSCs and ECM production as shown with the CAF related markers $\alpha$-SMA and Col1a1 (Figure 3, 4). In addition, ITGA11 knockdown significantly reduced the TGF- $\beta$ and Panc-1 CM-induced expression of PDGF $\beta$ R, Desmin, NSDHL, IDI1, INSIG-1, MMP-2 and CXCR-4. These markers were previously found to be induced in activated PSCs by transcriptome analysis ${ }^{8,34}$. Of high significance is the downregulation of ECM proteins e.g. collagens limiting chemotherapeutic activity by blocking drug delivery ${ }^{35,36}$. In addition, the gene profiler array for ECM and cell adhesion molecules revealed genes which were controlled by ITGA11, as shown by the knockdown of ITGA11. Among them, selectin, connectin, and TIMP3 were the top downregulated genes. Furthermore, we also revealed TGF- $\beta$-induced genes regulated by ITGA11 in hPSCs, and among them thrombospondin and CTGF, known to decrease the tumor response to gemcitabine ${ }^{37}$, were the most reduced ones (Table 5).

We further studied whether ITGA11 controls the function of hPSCs. Interestingly, ITGA11 knockdown resulted in a loss of cell-contractility or migration, with and without TGF- $\beta$ or Panc-1 CM mediated activation, of hPSCs. Overall, these data demonstrated that ITGA11 is crucial for the maintenance of the phenotypic features and activation of hPSCs.

Since hPSCs crosstalk with tumor cells and other stromal cells in the tumor microenvironment, we examined the effect of ITGA11 on the secretome of hPSCs. It has previously been demonstrated that hPSCs can induce tumorpromoting paracrine effects by secreting mutagenic factors such as growth factors and cytokines to induce tumor progression, invasion and metastasis 5,38 . In the present study, the knockdown of ITGA11 in quiescent hPSCs results in a phenotype with a reduced but also induced expression of tumor promoting factors. For example M-CSF, the most downregulated factor, is a cytokine which is known to be secreted by CAFs and promote the tumor-associated macrophage phenotype of monocytes in PDAC ${ }^{39}$. However, IL-6, the most upregulated factor, was shown to promote pancreatic cancer invasiveness (?) ${ }^{40}$. Upon activation of hPSCs with either TGF- $\beta$ or Panc-1 CM, various cytokines (CCL- 
22, PDGF-BB, MIF, IGFBP-1) were induced in shCTR hPSCs. Although not all the induced cytokines were controlled by ITGA11, there were a number of cytokines (MIF, IGFBP-1, CXCL1, TGF- $\beta 3$ ) whose secretion was reduced in shITGA11 hPSCs. Some of these factors are highly interesting in the context of pancreatic cancer progression. CXCL-1 has been identified to transform healthy fibroblasts into CAFs in an autocrine manner ${ }^{41}$, contributes to tumor progression by mediating interactions between cancer cells, tumor-associated macrophages and CAFs ${ }^{42}$ and contributes to radioresistance ${ }^{43}$. TGF- $\beta 3$ is known induce growth, migration and invasion on cancer cells ${ }^{44}$. PDGF-BB promoted stroma formation and tumor growth ${ }^{45}$. Interestingly, we also found that conditioned medium from shITGA11 hPSCs showed an attenuated migration and invasion of Panc-1 tumor cells compared to shCTR hPSCs. This might be due to TGF- $\beta 3$, known to induce the migration and invasion of cancer cells ${ }^{44}$, which is reduced in the conditioned medium of shITGA11 compared to shCTR hPSCs and is only induced with TGF- $\beta$ in shCTR but not in shITGA11 hPSCs.

Next to the reduced secretion of certain cytokines, there were also cytokines which were induced in shITGA11 hPSCs. However, with the exception of IGFBP-3, these cytokines were in the top six of cytokines found to be downregulated by ITGA11 knockdown, therefore their higher induction in TGF$\beta$ or Panc-1 CM activated shITGA11 compared to shCTR hPSCs might be due to a compensatory cellular mechanism, which re-induces the expression of these factors.

In conclusion, the present study for the first time demonstrates the expression and role of ITGA11 in pancreatic tumor stroma. ITGA11 was specifically and strongly expressed in the tumor stroma co-localized with CAFs. Furthermore, we show ITGA11 as a crucial target in maintenance of the phenotype and activation and differentiation of hPSCs. Nonetheless, our knockdown studies demonstrated a direct regulatory effect of ITGA11 on the transcriptome and secretome of activated hPSCs which controls their paracrine function. Altogether, ITGA11 is a promising therapeutic target for developing new therapeutics to inhibit the hPSC-mediated crosstalk in the tumor microenvironment.

\subsection{Financial supports}

The study was supported by the Swedish Research Council, Stockholm (project no. 2011-5389). 


\subsection{Conflicts of interest}

J Prakash is a founder and stakeholder of ScarTec Therapeutics BV. Enschede. The authors have no other relevant affiliations or financial involvement with any organization or entity with a financial interest in or financial conflict with the subject matter or materials discussed in the manuscript apart from those disclosed.

\subsection{Reverences}

1 Siegel, R. L., Miller, K. D. \& Jemal, A. Cancer Statistics, 2017. CA: a cancer journal for clinicians 67, 7-30, doi:10.3322/caac.21387 (2017).

2 Conroy, T. et al. FOLFIRINOX versus gemcitabine for metastatic pancreatic cancer. The New England journal of medicine 364, 18171825, doi:10.1056/NEJMoa1011923 (2011).

3 Von Hoff, D. D. et al. Increased survival in pancreatic cancer with nabpaclitaxel plus gemcitabine. The New England journal of medicine 369, 1691-1703, doi:10.1056/NEJMoa1304369 (2013).

4 Xie, D. \& Xie, K. Pancreatic cancer stromal biology and therapy. Genes Dis 2, 133-143, doi:10.1016/j.gendis.2015.01.002 (2015).

5 Hwang, R. F. et al. Cancer-associated stromal fibroblasts promote pancreatic tumor progression. Cancer research 68, 918-926, doi:10.1158/0008-5472.CAN-07-5714 (2008).

6 Apte, M. V. \& Wilson, J. S. Dangerous liaisons: pancreatic stellate cells and pancreatic cancer cells. Journal of gastroenterology and hepatology 27 Suppl 2, 69-74, doi:10.1111/j.1440-1746.2011.07000.x (2012).

7 Vonlaufen, A. et al. Pancreatic stellate cells: partners in crime with pancreatic cancer cells. Cancer research 68, 2085-2093, doi:10.1158/0008-5472.CAN-07-2477 (2008).

8 Sherman, M. H. et al. Vitamin D receptor-mediated stromal reprogramming suppresses pancreatitis and enhances pancreatic cancer therapy. Cell 159, 80-93, doi:10.1016/j.cell.2014.08.007 (2014).

9 Provenzano, P. P. et al. Enzymatic targeting of the stroma ablates physical barriers to treatment of pancreatic ductal adenocarcinoma. Cancer Cell 21, 418-429, doi:10.1016/j.ccr.2012.01.007 (2012).

10 Ozdemir, B. C. et al. Depletion of carcinoma-associated fibroblasts and fibrosis induces immunosuppression and accelerates pancreas cancer with reduced survival. Cancer cell 25, 719-734, doi:10.1016/j.ccr.2014.04.005 (2014).

11 Shields, M. A., Dangi-Garimella, S., Redig, A. J. \& Munshi, H. G. Biochemical role of the collagen-rich tumour microenvironment in pancreatic cancer progression. The Biochemical journal 441, 541-552, doi:10.1042/BJ20111240 (2012). 
12 Kuninty, P. R., Schnittert, J., Storm, G. \& Prakash, J. MicroRNA Targeting to Modulate Tumor Microenvironment. Front Oncol 6, 3, doi:10.3389/fonc.2016.00003 (2016).

13 Prakash, J. Cancer-Associated Fibroblasts: Perspectives in Cancer Therapy. Trends in cancer 2, 277-279, doi:10.1016/j.trecan.2016.04.005 (2016).

14 Bachem, M. G. et al. Identification, culture, and characterization of pancreatic stellate cells in rats and humans. Gastroenterology 115, 421432 (1998).

15 Habisch, H., Zhou, S., Siech, M. \& Bachem, M. G. Interaction of stellate cells with pancreatic carcinoma cells. Cancers (Basel) 2, 1661-1682, doi:10.3390/cancers2031661 (2010).

16 Apte, M. V., Wilson, J. S., Lugea, A. \& Pandol, S. J. A starring role for stellate cells in the pancreatic cancer microenvironment. Gastroenterology 144, 1210-1219, doi:10.1053/j.gastro.2012.11.037 (2013).

17 Bachem, M. G. et al. Pancreatic carcinoma cells induce fibrosis by stimulating proliferation and matrix synthesis of stellate cells. Gastroenterology 128, 907-921 (2005).

18 Schnittert, J., Bansal, R., Storm, G. \& Prakash, J. Integrins in wound healing, fibrosis and tumor stroma: High potential targets for therapeutics and drug delivery. Adv Drug Deliv Rev, doi:10.1016/j.addr.2018.01.020 (2018).

19 Seguin, L., Desgrosellier, J. S., Weis, S. M. \& Cheresh, D. A. Integrins and cancer: regulators of cancer stemness, metastasis, and drug resistance. Trends in cell biology 25, 234-240, doi:10.1016/j.tcb.2014.12.006 (2015).

20 Conroy, K. P., Kitto, L. J. \& Henderson, N. C. alphav integrins: key regulators of tissue fibrosis. Cell and tissue research 365, 511-519, doi:10.1007/s00441-016-2407-9 (2016).

21 Ivaska, J. \& Heino, J. Cooperation between integrins and growth factor receptors in signaling and endocytosis. Annu Rev Cell Dev Biol 27, 291320, doi:10.1146/annurev-cellbio-092910-154017 (2011).

22 Parajuli, H. et al. Integrin alpha11 is overexpressed by tumour stroma of head and neck squamous cell carcinoma and correlates positively with alpha smooth muscle actin expression. Journal of oral pathology \& medicine : official publication of the International Association of Oral Pathologists and the American Academy of Oral Pathology 46, 267-275, doi:10.1111/jop.12493 (2017).

23 Navab, R. et al. Integrin alpha11beta1 regulates cancer stromal stiffness and promotes tumorigenicity and metastasis in non-small cell lung cancer. Oncogene 35, 1899-1908, doi:10.1038/onc.2015.254 (2016). 
24 Bansal, R. et al. Integrin alpha 11 in the regulation of the myofibroblast phenotype: implications for fibrotic diseases. Experimental \& molecular medicine 49, e396, doi:10.1038/emm.2017.213 (2017).

25 Bansal, R. et al. PEGylation improves pharmacokinetic profile, liver uptake and efficacy of Interferon gamma in liver fibrosis. Journal of controlled release : official journal of the Controlled Release Society 154, 233-240, doi:10.1016/j.jconrel.2011.05.027 (2011).

26 Karagiannis, G. S. et al. Cancer-associated fibroblasts drive the progression of metastasis through both paracrine and mechanical pressure on cancer tissue. Molecular cancer research : MCR 10, 14031418, doi:10.1158/1541-7786.MCR-12-0307 (2012).

27 Yazdani, S., Bansal, R. \& Prakash, J. Drug targeting to myofibroblasts: Implications for fibrosis and cancer. Advanced drug delivery reviews 121, 101-116, doi:10.1016/j.addr.2017.07.010 (2017).

28 Kuninty, P. R. et al. MicroRNA-199a and -214 as potential therapeutic targets in pancreatic stellate cells in pancreatic tumor. Oncotarget 7, 16396-16408, doi:10.18632/oncotarget.7651 (2016).

29 Costa-Silva, B. et al. Pancreatic cancer exosomes initiate pre-metastatic niche formation in the liver. Nature cell biology 17, 816-826, doi:10.1038/ncb3169 (2015).

30 Sarper, M., Cortes, E., Lieberthal, T. J. \& Del Rio Hernandez, A. ATRA modulates mechanical activation of TGF-beta by pancreatic stellate cells. Sci Rep 6, 27639, doi:10.1038/srep27639 (2016).

31 Reed, N. I. et al. The alphavbeta1 integrin plays a critical in vivo role in tissue fibrosis. Sci Transl Med 7, 288ra279, doi:10.1126/scitranslmed.aaa5094 (2015).

32 Henderson, N. C. et al. Targeting of alphav integrin identifies a core molecular pathway that regulates fibrosis in several organs. Nature medicine 19, 1617-1624, doi:10.1038/nm.3282 (2013).

$33 \mathrm{Lu}, \mathrm{N}$. et al. The human alpha11 integrin promoter drives fibroblastrestricted expression in vivo and is regulated by TGF-beta1 in a Smadand Sp1-dependent manner. Matrix biology: journal of the International Society for Matrix Biology 29, 166-176, doi:10.1016/j.matbio.2009.11.003 (2010).

34 Kalluri, R. The biology and function of fibroblasts in cancer. Nature reviews. Cancer 16, 582-598, doi:10.1038/nrc.2016.73 (2016).

35 Minchinton, A. I. \& Tannock, I. F. Drug penetration in solid tumours. Nature reviews. Cancer 6, 583-592, doi:10.1038/nrc1893 (2006).

36 Netti, P. A., Berk, D. A., Swartz, M. A., Grodzinsky, A. J. \& Jain, R. K. Role of extracellular matrix assembly in interstitial transport in solid tumors. Cancer research 60, 2497-2503 (2000).

37 Neesse, A. et al. CTGF antagonism with mAb FG-3019 enhances chemotherapy response without increasing drug delivery in murine 
ductal pancreas cancer. Proc. Natl. Acad. Sci. U. S. A. 110, 1232512330, doi:10.1073/pnas.1300415110 (2013).

38 Apte, M. V., Wilson, J. S., Lugea, A. \& Pandol, S. J. A Starring Role for Stellate Cells in the Pancreatic Cancer Microenvironment. Gastroenterology 144, 1210-1219, doi:10.1053/j.gastro.2012.11.037 (2013).

39 Zhang, A. et al. Cancer-associated fibroblasts promote M2 polarization of macrophages in pancreatic ductal adenocarcinoma. Cancer medicine 6, 463-470, doi:10.1002/cam4.993 (2017).

40 Nagathihalli, N. S. et al. Pancreatic stellate cell secreted IL-6 stimulates STAT3 dependent invasiveness of pancreatic intraepithelial neoplasia and cancer cells. Oncotarget 7, 65982-65992, doi:10.18632/oncotarget.11786 (2016).

41 Kim, E. K., Moon, S., Kim, D. K., Zhang, X. \& Kim, J. CXCL1 induces senescence of cancer-associated fibroblasts via autocrine loops in oral squamous cell carcinoma. PLoS One 13, e0188847, doi:10.1371/journal.pone.0188847 (2018).

42 Miyake, M. et al. CXCL1-Mediated Interaction of Cancer Cells with Tumor-Associated Macrophages and Cancer-Associated Fibroblasts Promotes Tumor Progression in Human Bladder Cancer. Neoplasia 18, 636-646, doi:10.1016/j.neo.2016.08.002 (2016).

43 Zhang, H. et al. CAF-secreted CXCL1 conferred radioresistance by regulating DNA damage response in a ROS-dependent manner in esophageal squamous cell carcinoma. Cell death \& disease 8, e2790, doi:10.1038/cddis.2017.180 (2017).

44 Qin, X. et al. TGFbeta3-mediated induction of Periostin facilitates head and neck cancer growth and is associated with metastasis. Scientific reports 6, 20587, doi:10.1038/srep20587 (2016).

45 Heldin, C. H. Targeting the PDGF signaling pathway in tumor treatment. Cell communication and signaling : CCS 11, 97, doi:10.1186/1478811X-11-97 (2013).

\subsection{Supplementary}

Supplementary Table 1. Cytokines and growth factors downregulated or upregulated in shITGA11 hPSCs compared with shCTR. The data was obtained with the Human Cytokine Antibody Array C5 (RayBioß) and quantified using ImageJ.

\begin{tabular}{lll}
\hline Protein ID & Description & Fold Regulation \\
\hline M-CSF & Macrophage colony-stimulating factor & $-2,874093954$ \\
Angiogenin & Angiogenin & $-2,088758183$ \\
CCL4 & C-C motif chemokine 4 & $-1,890804333$ \\
TGF-b 3 & Transforming growth factor beta 3 & $-1,832072346$
\end{tabular}




\begin{tabular}{|c|c|c|}
\hline CCL22 & C-C motif chemokine 22 & $-1,825996084$ \\
\hline LIF & Leukemia inhibitory factor & $-1,539654607$ \\
\hline VEGF-A & Vascular endothelial growth factor A & $-1,455564159$ \\
\hline TNF beta & Tumor necrosis factor & $-1,360741111$ \\
\hline $\mathrm{GRO} \mathrm{a} / \mathrm{b} / \mathrm{g}$ & Oncogene GRO a/b/g & $-1,275876741$ \\
\hline BDNF & Brain-derived neurotrophic factor & $-1,183278706$ \\
\hline HGF & Hepatocyte growth factor & $-1,15222553$ \\
\hline TIMP-1 & Tissue inhibitor of metalloproteases & $-1,110709638$ \\
\hline CCL-17 & $\mathrm{C}-\mathrm{C}$ motif chemokine ligand 17 & $-1,080764946$ \\
\hline IP-10 & $\mathrm{C}-\mathrm{X}-\mathrm{C}$ motif chemokine 10 & $-1,061076338$ \\
\hline OSM & Oncostatin-M & $-1,043949116$ \\
\hline EGF & Epidermal growth factor & $-1,021746053$ \\
\hline TIMP-2 & Metalloproteinase inhibitor 2 & 1,019660243 \\
\hline IL-8 & Interleukin-8 & 1,030393099 \\
\hline PDGF-BB & Platelet-derived growth factor subunit B & 1,143836841 \\
\hline IGFBP-2 & Insulin-like growth factor-binding protein 2 & 1,204848312 \\
\hline CXCL5 & $\mathrm{C}-\mathrm{X}-\mathrm{C}$ motif chemokine 5 & 1,223342142 \\
\hline TGF-b 2 & Transforming growth factor beta 2 & 1,238059015 \\
\hline IGFBP-3 & Insulin-like growth factor-binding protein 3 & 1,24913089 \\
\hline $\mathrm{CCl} 23$ & $\mathrm{C}-\mathrm{C}$ motif chemokine 23 & 1,311194625 \\
\hline IL-7 & Interleukin-7 & 1,33322543 \\
\hline OPN & Osteopontin & 1,564699424 \\
\hline CXCL1 & $\mathrm{C}-\mathrm{X}-\mathrm{C}$ motif chemokine 1 & 1,669274325 \\
\hline FGF-9 & Fibroblast growth factor 9 & 1,706309402 \\
\hline IGF-1 & Insulin-like growth factor I & 1,730488364 \\
\hline FGF-7 & Fibroblast growth factor 7 & 1,731072065 \\
\hline IGFBP-1 & Insulin-like growth factor-binding protein 1 & 1,758058869 \\
\hline FGF-6 & Fibroblast growth factor 6 & 1,891364755 \\
\hline CCL2 & C-C motif chemokine 2 & 2,05651861 \\
\hline MIF & Macrophage migration inhibitory factor & 2,149189951 \\
\hline OPG & Osteoprotegerin & 2,486250312 \\
\hline IL-6 & Interleukin-6 & 3,061058772 \\
\hline
\end{tabular}


Chapter 7 (page 199-224) under embargo until 31-12-2023 


\section{Chapter 8 - Summary and Discussion}

Pancreatic ductal adenocarcinoma (PDAC) is the cancer type with the worst prognosis. The 1-year survival rate is $20 \%$, whereas the 5 -year survival rate is $8 \%{ }^{1}$. A surgical resection is only feasible in a small percentage of PDAC tumors, making radiotherapy or cytotoxic chemotherapy the golden standard treatment. A lot of research has been conducted in search of more effective therapies for PDAC. However, only marginal improvements have been made with regard to the survival rate ${ }^{2-4}$. The abundant desmoplasia in PDAC, also called the tumor stroma or tumor microenvironment, which supports cancer progression, invasion and metastasis, is one of the main causes for treatment failure ${ }^{5}$.

Abundant components of the tumor stroma include cancer-associated fibroblasts (CAFs), tumor-associated macrophages (TAMs), pericytes, endothelial cells, infiltrating immune cells (e.g. T-cells, natural killer (NK) cells and macrophages) and ECM proteins (e.g. collagen) ${ }^{6}$. CAFs are the key drivers of the pancreatic tumor stroma, with their main precursors, human pancreatic stellate cells (hPSCs) ${ }^{6-8}$. Quiescent hPSCs are known for their vitamin A storing ability, can be found within the connective tissue of the pancreas in low numbers and secrete only small amounts of extracellular matrix (ECM) proteins (e.g. collagen) 9 . During PDAC progression, quiescent hPSCs become activated into CAF-like myofibroblasts as response to tissue injury and growth factors such as TGF- $\beta 1$. As a result, they lose their cytoplasmic lipid storing capacity and start secreting high amounts of ECM components which leads to desmoplasia. Desmoplasia, fibrotic stromal tissue, acts as a physical barrier, preventing drug accumulation in the tumor tissue ${ }^{10,11}$. Additionally, activated hPSCs secrete various cytokines and growth factors, which stimulate tumor cells, thereby facilitating tumor growth, invasion and metastasis and ultimately tumor progression 12,13. Therefore, modulation of the tumor stroma by dampening the function of activated hPSCs presents an innovative approach to gain therapeutic benefits and improve the treatment of PDAC.

In this thesis, we focused on the identification of new therapeutic targets, including microRNA (miRNA), integrin receptors and bioactive lipids, to reprogram activated hPSCs into their quiescent phenotype and thereby modulate the tumor stroma in pancreatic cancer. Furthermore, we designed a novel system for miRNA delivery into hPSC and designed an integrin alpha 11 specific peptide for targeting of drug delivery systems to activated hPSCs in the stroma of PDAC. 
In chapter 2, we introduce miRNAs in the tumor stroma, which have emerged as key players involved in the development of cancer and its progression. MicroRNAs present a potential class of therapeutics, which regulate various signaling pathways within the tumor microenvironment ${ }^{14}$. In general, miRNAs are a class of small (17-25 nt), endogenous, single stranded non-coding RNAs, which control gene expression at the post-transcriptional level and thereby control various cellular processes ${ }^{15}$. A unique ability of miRNAs is their ability to regulate not only one but hundreds of target genes simultaneously, enabling them to control multiple signaling pathways ${ }^{16}$. This is possible, because miRNAs silence genes via imperfect and/or perfect complementary base pairing between a miRNA guide strand and the 3' UTR region of the target mRNA, which causes translational repression or mRNA degradation ${ }^{15,17}$. In a pathological state such as cancer, the expression levels of multiple miRNAs are aberrantly up- or downregulated, resulting in an imbalance of cellular pathways. In chapter 2, we describe dysregulated miRNAs in cells of the tumor stroma and their functions in the regulation of the tumor microenvironment. Additionally, we describe how dysregulated miRNA levels can be controlled by administering miRNA inhibitors (anti-miRNA oligonucleotides) or miRNA agonists (miRNA mimics). Finally, we summarize various miRNA delivery approaches that have been or can potentially be applied to deliver miRNA as therapeutics into stromal cells.

In chapter 3, we present a novel peptide based miRNA delivery system to deliver anti-miRNA oligonucleotides (AMOs) to hPSCs. A dimeric form of an arginine- rich cell penetrating peptide (CPP) was synthesized and used for preparing self-assembling nanocomplexes (NCs) with AMOs, via charge-charge interactions. Intriguingly, these NCs showed significantly higher uptake by hPSCs compared with the NCs prepared with the monomeric form. Although branching or multivariant approaches may enhance CPP-mediated uptake, our dimeric CPP-based NCs were preferentially internalized in hPSCs compared with normal fibroblasts or pancreatic tumor cells. This led us to examine different uptake mechanisms, by which hPSCs take up these NCs, showing their uptake via receptor-mediated endocytosis. In our lab, we have previously identified miRNA-199a as a novel therapeutic target in CAFs of PDAC patients and demonstrated that miRNA-199a was induced in patient-derived CAFs as well as activated hPSCs ${ }^{18}$. Furthermore miRNA-199a was shown to regulate the activation of hPSCs and hPSC-induced tumor promoting effects on tumor cells ${ }^{18}$. Therefore, we utilized our NCs for specific delivery of an AMO against miR199a to hPSCs in 2D cell cultures and 3D-heterospheroids. Interestingly, 
delivery of anti-miR-199a into hPSCs inhibited their TGF- $\beta$-mediated activation and hPSC-mediated paracrine effects on tumor cell growth.

In chapter 4, we looked into inflammation resolving bioactive lipids as potential therapeutics to dampen the function of CAFs and thereby reprogram the tumor stroma. We identified lipoxin A4 (LXA4), a highly potent endogenous lipid, known for its anti-inflammatory and anti-fibrotic function as a potential therapeutic to inhibit the tumor promoting function of activated hPSC ${ }^{19-21}$. We showed that The LXA4-specific surface receptor FPR2/ALX was upregulated in TGF $\beta$ activated hPSCs. Treatment of hPSCs with LXA4 inhibited TGF- $\beta$ mediated induction of hPSC activation markers (e.g. $\alpha$ SMA, collagen, PDGFR $\beta$ ), migration and morphological changes in hPSC and therefore the activation of hPSCs into CAF-like myofibroblasts in vitro. Additionally, LXA4 inhibited hPSC-induced tumor-promoting paracrine effects, including tumor cell proliferation and migration. In our novel 3D-heterospheroidal in vitro tumor model, consisting of hPSCs and Panc-1 tumor cells, LXA4 could inhibit the activation of hPSCs within the 3D microenvironment and reduced spheroid growth and ECM deposition within the spheroids. Finally, we showed the therapeutic efficacy of LXA4 in vivo in an hPSC/Panc-1 co-injection tumor model, in which LXA4 reduced tumor growth and ECM-deposition. This study highlights LXA4 as a potential biological to be developed for the treatment of pancreatic cancer.

Next to miRNA and bioactive lipids, we investigated integrins as therapeutic and drug delivery targets, since integrin signaling is critical for cell activation (differentiation), an integration of signaling networks with a role in tumor progression, invasion and metastasis ${ }^{22,23}$. In Chapter 5 we provide a general introduction of integrins and integrin-mediated signaling, an overview of integrin expression in fibrosis-related cell types, and the interaction between integrins and growth factor receptors. The next section describes the role of integrins in wound healing, fibrosis and tumor stroma. The final two sections are focused on the novel therapies based on the inhibition of integrin receptors, including clinical developments, and drug delivery strategies to target integrins. We find implications for various novel therapeutic and drug targeting targets in studies which revealed the biological function of various integrins within fibrosis related disease. However, we conclude that studies exploiting the expression of a specific integrin subunit and its cellular localization within pathological tissues and other healthy organs will be crucial to determine novel integrin-based therapeutic targets. Moreover, the design of highly integrin subunit specific 
drugs will decrease the risk of off-target effects due to an increased specificity of these molecules. A clear understanding of integrin subunit - growth factor receptor interactions is an additional major aspects to identify integrin subunits which can be utilized as therapeutic targets.

Chapter 6 introduces the collagen 1 binding transmembrane receptor ITGA11 as a significant regulator of the phenotype and function of CAFs. ITGA11 was shown to be highly induced in the stroma of human PDAC and co-localized with the major CAF marker, $\alpha$-SMA, in PDAC samples. Strikingly, the knockdown of ITGA11 in hPSCs inhibited their TGF- $\beta$-mediated activation, migration and contractility. A gene profiler array for ECM and adhesion molecules and a cytokine array, revealed that many of these factors were downregulated in hPSCs after ITGA11 knockdown or significantly less induced in activated hPSCs with shITGA11 knockdown. Moreover, we show that the knockdown of ITGA11 inhibits hPSCs mediated paracrine effects on Panc-1 tumor cell migration and invasion. In conclusion, ITGA11 was found to be highly and specifically expressed in the tumor stroma of PDAC and this study presents ITGA11 as a crucial target in maintaining the phenotype, activation ability and function of hPSCs. Therefore ITGA11 is expected to have a strong impact on the regulation of hPSC-induced desmoplasia in PDAC.

Activated hPSCs also known as CAFs, significantly contribute to the development of the dense tumor stroma, which prevents drug penetration and thereby causes drug resistance in PDAC. Therefore, drug delivery to pancreatic tumors by targeting CAFs, presents a promising approach to specifically delivery therapeutics to CAFs, thereby modulate the tumor stroma and enhance drug penetration into the tumor. However, no CAF-specific targets have been previously reported ${ }^{24}$. In chapter 7 we have identified the integrin subunit ITGA11, to be specifically expressed on CAFs in the tumor stroma of PDAC. Using phage display we have designed a novel peptide (AXI), specifically binding to ITGA11. We use AXI to deliver liposomes to CAFs in vitro and show that AXI binds to ITGA11 on hPSCs, thereby facilitating the uptake of AXIdecorated liposomes into these cells. In vivo, liposomes modified with AXI on their surface showed enhanced accumulation and penetration into the tissue of ITGA11 rich hPSC + Panc-1 tumor bearing mice and increased accumulation of liposomes in ITGA11 positive cells, when compared to liposomes modified with a scrambled version of AXI. In conclusion, this study presents AXI, a novel ITGA11 targeting peptide, which can be utilized for targeted drug delivery to reprogram CAFs and thereby modulate the tumor stroma in pancreatic cancer. 
In conclusion, there is an urgent need for novel therapeutic strategies to treat PDAC, since traditional chemotherapies aiming to kill cancer cells are failing to cure PDAC in the clinic. Therefore, this thesis explores strategies to modulate and actively target the tumor stroma in PDAC, which is a rapidly emerging area in oncology. In the context of the tumor stroma we focused on hPSCs, which significantly contribute to tumor progression in PDAC. We show the therapeutic potential of anti-miR-199a oligonucleotides (in vitro) and LXA4 (in vitro and in vivo) to reprogram activated hPSCs and thereby their tumor promoting effects. Additionally, we introduce a novel system to deliver anti-miRNA into hPSCs. Next, we identified ITGA11 as a novel therapeutic target specifically expressed on CAFs in PDAC, which controls the phenotype and function of these cells. We introduce ITGA11 as a high potential target for drug delivery, due to its specific expression in the tumor stroma, which minimizes the risk of off-target effects. Finally, we designed an ITGA11 targeting peptide which showed increased accumulation and penetration depth and the ability to localize in ITGA11expressing CAFs in vivo. For future studies it is highly recommended to combine the CAF-reprogramming therapeutics and drug targeting strategies discovered in this thesis and investigate their effect as combination treatment with chemotherapeutics.

\section{References}

1 Siegel, R. L., Miller, K. D. \& Jemal, A. Cancer statistics, 2018. CA: a cancer journal for clinicians 68, 7-30, doi:10.3322/caac.21442 (2018).

2 Hidalgo, M. Pancreatic cancer. The New England journal of medicine 362, 1605-1617, doi:10.1056/NEJMra0901557 (2010).

3 Maitra, A. \& Hruban, R. H. Pancreatic cancer. Annual review of pathology $\quad \mathbf{3}, \quad$ 157-188, doi:10.1146/annurev.pathmechdis.3.121806.154305 (2008).

4 Liu, Q., Liao, Q. \& Zhao, Y. Chemotherapy and tumor microenvironment of pancreatic cancer. Cancer cell international 17, 68, doi:10.1186/s12935-017-0437-3 (2017).

5 Xie, D. \& Xie, K. Pancreatic cancer stromal biology and therapy. Genes \& diseases 2, 133-143, doi:10.1016/j.gendis.2015.01.002 (2015).

6 Kuninty, P. R., Schnittert, J., Storm, G. \& Prakash, J. MicroRNA Targeting to Modulate Tumor Microenvironment. Front Oncol 6, 3, doi:10.3389/fonc.2016.00003 (2016).

7 Shields, M. A., Dangi-Garimella, S., Redig, A. J. \& Munshi, H. G. Biochemical role of the collagen-rich tumour microenvironment in pancreatic cancer progression. The Biochemical journal 441, 541-552, doi:10.1042/BJ20111240 (2012). 
8 Sherman, M. H. et al. Vitamin D receptor-mediated stromal reprogramming suppresses pancreatitis and enhances pancreatic cancer therapy. Cell 159, 80-93, doi:10.1016/j.cell.2014.08.007 (2014).

9 Habisch, H., Zhou, S., Siech, M. \& Bachem, M. G. Interaction of stellate cells with pancreatic carcinoma cells. Cancers (Basel) 2, 1661-1682, doi:10.3390/cancers2031661 (2010).

10 Jacobetz, M. A. et al. Hyaluronan impairs vascular function and drug delivery in a mouse model of pancreatic cancer. Gut 62, 112-120, doi:10.1136/gutjnl-2012-302529 (2013).

11 Provenzano, P. P. et al. Enzymatic targeting of the stroma ablates physical barriers to treatment of pancreatic ductal adenocarcinoma. Cancer cell 21, 418-429, doi:10.1016/j.ccr.2012.01.007 (2012).

12 Apte, M. V., Wilson, J. S., Lugea, A. \& Pandol, S. J. A starring role for stellate cells in the pancreatic cancer microenvironment. Gastroenterology 144, 1210-1219, doi:10.1053/j.gastro.2012.11.037 (2013).

13 Feig, C. et al. The pancreas cancer microenvironment. Clinical cancer research : an official journal of the American Association for Cancer Research 18, 4266-4276, doi:10.1158/1078-0432.CCR-11-3114 (2012).

14 Suzuki, H. I., Katsura, A., Matsuyama, H. \& Miyazono, K. MicroRNA regulons in tumor microenvironment. Oncogene 34, 3085-3094, doi:10.1038/onc.2014.254 (2015).

15 Melo, S. A. \& Kalluri, R. Molecular pathways: microRNAs as cancer therapeutics. Clinical cancer research : an official journal of the American Association for Cancer Research 18, 4234-4239, doi:10.1158/1078-0432.CCR-11-2010 (2012).

16 Esquela-Kerscher, A. \& Slack, F. J. Oncomirs - microRNAs with a role in cancer. Nature reviews. Cancer 6, 259-269, doi:10.1038/nrc1840 (2006).

17 Bader, A. G., Brown, D., Stoudemire, J. \& Lammers, P. Developing therapeutic microRNAs for cancer. Gene therapy 18, 1121-1126, doi:10.1038/gt.2011.79 (2011).

18 Kuninty, P. R. et al. MicroRNA-199a and -214 as potential therapeutic targets in pancreatic stellate cells in pancreatic tumor. Oncotarget 7, 16396-16408, doi:10.18632/oncotarget.7651 (2016).

19 Mitchell, D. et al. Lipoxins inhibit Akt/PKB activation and cell cycle progression in human mesangial cells. The American journal of pathology 164, 937-946, doi:10.1016/S0002-9440(10)63181-1 (2004).

20 Borgeson, E. et al. Lipoxin A(4) and benzo-lipoxin A(4) attenuate experimental renal fibrosis. FASEB journal : official publication of the Federation of American Societies for Experimental Biology 25, 29672979, doi:10.1096/fj.11-185017 (2011).

21 Roach, K. M., Feghali-Bostwick, C. A., Amrani, Y. \& Bradding, P. Lipoxin A4 Attenuates Constitutive and TGF-beta1-Dependent 
Profibrotic Activity in Human Lung Myofibroblasts. J Immunol 195, 2852-2860, doi:10.4049/jimmunol.1500936 (2015).

22 Guo, W. \& Giancotti, F. G. Integrin signalling during tumour progression. Nature reviews. Molecular cell biology 5, 816-826, doi:10.1038/nrm1490 (2004).

23 Hood, J. D. \& Cheresh, D. A. Role of integrins in cell invasion and migration. Nature reviews. Cancer 2, 91-100, doi:10.1038/nrc727 (2002).

24 Schnittert, J., Bansal, R., Storm, G. \& Prakash, J. Integrins in wound healing, fibrosis and tumor stroma: High potential targets for therapeutics and drug delivery. Advanced drug delivery reviews, doi:10.1016/j.addr.2018.01.020 (2018). 


\section{Appendix A - Nederlandse Samenvatting}

Pancreatic ductal adenocarcinoma (PDAC) is het type kanker met de slechtste prognose met een 1-jarigoverlevingspercentage van $20 \%$ en een5-jarige overlevingspercentage van slechts $8 \%$. Chirurgische resectie is alleen bij een klein percentage van patiënten mogelijk en daardoor maakt dit radio- of chemotherapie de stadaard behandelingsmethode. $\mathrm{Er}$ is veel onderzoek uitgevoerd naar het vinden van effectieve behandelmethodes voor PDAC. Tot nu toe zijn er echter alleen marginale verbeteringen in het overlevingspercentage bereikt. Eén van de hoofdoorzaken van het falen van de behandeling van PDAC is de overvloedige desmoplasie in PDAC, ook wel tumor stroma of tumor micro-omgeving genoemd, die de PDAC progressie, invasie en de vorming van metastasenondersteunt

Veel voorkomende componenten van het tumor stroma zijn kankergeassocieerde fibroblasten (CAFs), tumor-geassocieerde macrofagen (TAMs), pericyten, endotheelcellen, infiltrerende immuuncellen en eiwitten van de extracellulaire matrix (ECM) (b.v. collageen). Eén van de meest voorkomende celtypen uit de pancreas tumor stroma zijn CAFs. De voorlopers van CAFs zijn human pancreatic stellate cells (hPSCs). 'Quiescent' hPSCs staan bekend om hun vermogen om vitamine A op te slaan, zijn in kleine hoeveelheden in het bindweefsel van de pancreas te vinden en produceren alleen kleine hoeveelheden van ECM eiwitten. Tijdens PDAC progressie, worden 'quiescent' hPSCs, door weefsel schade en groeifactoren zoals TGF- $\beta 1$, in CAF-achtige myofibroblasten geactiveerd. Als gevolg hiervan verliezen zij de capaciteit om lipiden op te slaan en starten met het produceren vangrote hoeveelheden ECM componenten, wat tot desmoplasie leidt. Desmoplasie, een fibrotisch stromaal weefsel, werkt als een fysieke barrière en voorkomt de accumulatie van medicijnen in de tumor. Daarnaast produceren geactiveerde hPSCs verschillende cytokines en groeifactoren, die tumorcellen stimuleren en hierdoor tumorgroei, invasie, metastase en uiteindelijk tumorprogressie faciliteren. Daarom is modulatie van het tumor stroma door het remmen van de functie van geactiveerde hPSCs een innovatieve strategie om de therapie van PDAC te verbeteren.

In dit proefschrift ligt de focus op de identificatie van nieuwe therapeutische 'targets', zoals microRNA (miRNA), integrine receptoren en bioactieve lipiden om geactiveerde hPSCs in hun 'quiescent' fenotype te reprogrammeren en hierdoor de tumor stroma van PDAC te moduleren. Bovendien hebben wij een 
nieuw systeem voor de afgifte van miRNA aan hPSCs en een integrine alpha 11 specifieke peptide voor de doelgerichte afgifte van medicijnen aan geactiveerde hPSCs in het stroma van PDAC ontwikkeld.

In hoofdstuk 2 introduceren wij het concept van miRNA ontregeling in de tumor stroma, welke een hoofdrol in de ontwikkeling en de progressie van kanker speelt en verschillende signaleringsroutes in het tumor stroma reguleert. Hierdoor zijn anti-miRNAs een potentiële klasse van medicijnen. In dit hoofdstuk beschrijven we ontregelde miRNAs in cellen van het tumor stroma en discussiëren we strategieën voor de doelgerichte afgifte van anti-miRNAs aan deze celtypes.

MicroRNAs zijn op zichzelf niet in staat om met de celmembraan te associëren en worden daarom ook niet door een cel opgenomen. In hoofdstuk 3 stellen wij een nieuw peptide gebaseerd miRNA afgifte systeem voor om anti-miRNA oligonucleotiden (AMOs) aan hPSCs af te geven. Hiervoor hebben wij nanocomplexen (NCs) met AMOs en een door ons ontworpen cel-penetrerend peptide (CPP) gevormd. Deze NCs vertonen significant meer opname in hPSCs indien vergeleken met alvleesklier tumor cellen en normale fibroblasten. Door de afgifte van anti-miR-199a met dit system werd de differentiatie van hPSCs in CAFs, hPSC-induceerde tumorcel groei, de groei van tumor sferoïden bestaande uit hPSCs en tumor cellen en hPSC-gebaseerde tumorcel groei geremd.

In hoofdstuk 4 hebben wij de ontstekingsremmende bioactieve lipide lipoxin A4 (LXA4) als potentieel potentiëe medicijn voor het remmen van de functie van CAFs getest. Behandeling van hPSCs met LXA4 remt hun activatie richting CAFs, gezien aan de verminderde expressie van activatiemarkers, de migratie potentiaal en morfologische veranderingen van hPSCs. Bovendien remt LXA4 tumor-bevorderende effecten van hPSCs, zoals tumorcel groei en migratie. In tumor sferoïden bestaande uit hPSCs en tumorcellen was LXA4 in staat on de activatie van hPSCs in de sferoïden evenals de groei van en ECM depositie in de sferoïden te remmen. Uiteindelijk laten wij de therapeutische werking van LXA4 in een hPSC/tumor cel co-injectie model in vivo zien, wat tot verminderde tumorgroei en ECM-depositie in de tumoren leidt. Deze studie accentueert LXA4 als een potentiële 'biological' voor de behandeling van PDAC.

Naast miRNA en bioactieve lipides hebben wij integrine als therapeutische en 'drug delivery targets' onderzocht, omdat integrine signalering kritisch is voor 
celactivatie, differentiatie en de integratie van cel signaleringsroutes met een rol in tumor progressie, invasie en metastase. In hoofdstuk 5 geven wij een algemene introductie over integrineexpressie in fibrose-gerelateerde celtypes en de interactie tussen integrines en groeifactor receptoren. De volgende sectie beschrijft de rol van integrines in wond genezing, fibrose en de tumor stroma. De laatste twee secties zijn gericht op nieuwe integrine-remmende therapieën, inclusief klinische ontwikkelingen en systemen voor de doelgerichte afgifte van medicijnen aan integrines. Wij concluderen dat de ontwikkeling van integrine-subunit specifieke medicijnen het risico van off-target effecten zou minimaliseren. Een duidelijk begrip van integrine subunit - groeifactor receptor interacties is een ander belangrijk aspect om integrine-subunits te identificeren, welke als therapeutische targets benut kunnen worden.

In hoofdstuk 6 stellen wij de transmembraanreceptor integrin alpha 11 (ITGA11) als regulator van de fenotype van CAFs voor. ITGA11 heeft een hoge expressie in het stroma van PDAC en is gelokaliseerd op CAFs. Het uitschakelen van ITGA11 in hPSCs remt hun activatie, migratie en contractie vermogen. Veel ECM- en celadhesiemoleculen komen in hPSCs met uitgeschakeld ITGA11 minder tot expressie of zijn minder geïnduceerd in geactiveerde hPSCs met uitgeschakeld ITGA11. Bovendien laten wij zien dat het uitschakelen van ITGA11 hPSC-geïnduceerd effecten op tumorcellen, zoals migratie en invasieve remt. Concluderend hebben wij gevonden dat ITGA11 verhoogd en specifiek in de stroma van PDAC tot expressie komt en een belangrijke target is in het behoud van het fenotype, activatiepotentiaal en functie van hPSCs. Hierdoor wordt verwacht dat ITGA11 een sterke rol in de regulatie van hPSC-geïnduceerde desmoplasie in PDAC speelt.

Geactiveerde hPSCs, ook bekend als CAFs, dragen significant aan de ontwikkeling van de tumor stroma in PDAC bij, wat de penetratie van medicijnen in het stroma vermindert. Daarom is de doelgericht medicijnafgifte aan CAFs in PDAC tumoren een veelbelovende strategie om de tumor stroma te moduleren en de penetratie van medicijnen in de stroma te verhogen. Er zijn echter nog geen CAF-specifieke targets bekend.

In hoofdstuk 7 hebben wij ITGA11 als specifieke CAF-marker in de tumor stroma van PDAC geïdentificeerd. Met behulp van de 'phage display' techniek hebben wij een nieuw peptide (AXI), met specifieke bindingsaffiniteit voor ITGA11 ontwikkeld. Wij hebben AXI gebruikt om liposomen via ITGA11 aan CAFs af te geven. Hierbij wordt de opname van AXI-gemodificeerde 
liposomen door ITGA11 faciliteert. In vivo laten AXI-gemodificeerde liposomen, vergeleken met liposomen gemodificeerd met een 'scrambled' versie van AXI (sAXI), een verhoogde accumulatie en penetratie in tumoren in muizen en ITGA11-positieve cellen zien. Concluderend hebben wij in deze studie een nieuwe ITGA11 'targeting' peptide ontwikkeld, welke voor de doelgerichte afgifte van medicijnen voor het reprogrammeren van CAFs en het moduleren van de tumor stroma in PDAC gebruikt kan worden.

Samenvattend is er een dringende behoefte voor nieuwe therapeutische strategieën voor de behandeling van PDAC, omdat traditioneel gebruikte chemotherapeutische therapieën falen om patiënten met PDAC te genezen. Daarom worden in dit proefschrift strategieën voor de modulatie van en doelgerichte medicijnafgifte aan de tumor stroma in PDAC onderzocht. In toekomstige studies wordt aanbevolen de CAF-reprogrammerende medicijnen en strategieën voor doelgerichte medicijnafgifte, ontdekt in dit proefschrift, te combineren en hun effect als combinatie behandeling met chemotherapie te evalueren. 


\section{Appendix B}

\section{List of Publications}

\section{Manuscript in preparation:}

"A novel peptide against integrin alpha11 to target nanoparticles to cancer-associated fibroblasts."

Schnittert J et al.

"Integrin $\alpha 11$ regulates differentiation of pancreatic stellate cells in pancreatic cancer."

Schnittert J et al.

"Pancreatic stellate cells: Are they the stars of pancreatic cancer?" Schnittert J et al.

\section{Submitted}

"Targeting integrin alpha5 receptor in pancreatic stellate cells to diminish tumor-promoting effects in pancreatic cancer"

Kuninty PR, Bansal R, De Geus SWL, Schnittert J, van Baarlen J, Storm G, Bijlsma MF, van Laarhoven HW, Kuppen PJK, Vahrmeijer AL, Ostman A, Sier CFM, Prakash J

Submitted to Nature Communications

\section{Published:}

"Integrins in wound healing, fibrosis and tumor stroma: High potential targets for therapeutics and drug delivery."

Schnittert J, Bansal R, Storm G, Prakash J.

Adv Drug Deliv Rev. 2018 Feb 4. pii: S0169-409X(18)30028-0. doi:

10.1016/j.addr.2018.01.020.

PMID: 29414674

"Reprogramming tumor stroma using an endogenous lipid lipoxin A4 to treat pancreatic cancer."

Schnittert J, Heinrich MA, Kuninty PR, Storm G, Prakash J.

Cancer Lett. 2018 Feb 2. pii: S0304-3835(18)30105-8. doi:

10.1016/j.canlet.2018.01.072.

PMID: 29408203 
"Anti-microRNA targeting using peptide-based nanocomplexes to inhibit differentiation of human pancreatic stellate cells."

Schnittert J, Kuninty PR, Bystry TF, Brock R, Storm G, Prakash J.

Nanomedicine (Lond). 2017 May 19. doi: 10.2217/nnm-2017-0054.

PMID: 28524768

"Stroma-regulated HMGA2 is an independent prognostic marker in PDAC and AAC."

Strell C, Norberg KJ, Mezheyeuski A, Schnittert J, Kuninty PR, Moro CF, Paulsson J, Schultz NA, Calatayud D, Löhr JM, Frings O, Verbeke CS, Heuchel RL, Prakash J, Johansen JS, Östman A.

Br J Cancer. 2017 Jun 27;117(1):65-77. doi: 10.1038/bjc.2017.140. Epub 2017 May 18.

PMID: 28524160

"MicroRNA Targeting to Modulate Tumor Microenvironment." Kuninty PR, Schnittert J, Storm G, Prakash J.

Front Oncol. 2016 Jan 19;6:3. doi: 10.3389/fonc.2016.00003. eCollection 2016. Review.

PMID: 26835418

\section{Scientific Awards}

Oral Free Paper Price

United European Gastroentereology (UEG) Week, October 2017

Best Poster / Small Speech Award

Annual meeting of CLINAM (European Foundation for Clinical

Nanomedicine), June 2016 


\section{Appendix C - Acknowledgements}

After almost 3.5 years I am now spending one of the last evening in my office $\mathrm{ZH} 253$ and have a very hard time to believe that the time has come to write this important acknowledgment section of my thesis. All this time went unbelievably quick. My plan was to keep it short. That did not work.

Foremost, I want to express my gratitude to Dr. Jai Prakash for enabling me to do this amazing project! You are the busiest person I know but always took time to give advice no matter the issues. You also gave me a lot of freedom and allowed me to develop. I have learned a lot from your scientific mentorship and will never forget the good times, scientific and private talks we had at work and during conferences. It was a real pleasure to work with you! If anyone I know deserves a 48 hour day it is you and Ruchi.

Dr. Ruchi Bansal, whether it was intellectual or practical input thank you for always being supportive and the extremely nice person that you are. To me you are such an important part of our group!

Prof. Dr. Gert Strom, first of all I want to thank you for proposing Jai that I could be a suitable candidate for his project while we had coffee together almost 4 years ago. I enjoyed the motivating and fun conversations which had over the last 3.5 years and really admire your entertaining but at the same time serious and critical personality.

My gratitude also goes to the members of my evaluation committee Prof. Dr. P. Jonkheijm, Prof. Dr. M. van de Laar, Prof. Dr. J. Klaase, Prof. Dr. R. Brock and Prof. Dr. Y. van Kooyk for all the time and effort you put in evaluating my thesis and taking part in the defense ceremony.

Praneeth, I got to know you as my supervisor during my master thesis and you have become one of my best buddies at Targeted Therapeutics. We have fought through good and bad times together over the last 4 years and although our start was not the easiest we have become a very good team. I really enjoyed working with you and I am happy that you were there. Thanks for being the funny person you are, always taking care of the social gathering of Targeted Therapeutics and supporting me today. The two of us should definitely still go Scuba Diving ;-). Yes!? 
Marcel, besides having my old master thesis supervisor by my side today it is a great pleasure to have you, my old master student as a paranymph. You were the best student I could think of and I am happy that you became my colleague and most importantly friend! No matter the issue, I could discuss everything with you and I will not forget the fun times we had in ZH253. I am certain that you will become a brilliant and successful scientist and now that I cannot distract you anymore your productivity will likely go through the roof ;-).

A big thanks to Dhadhang, my other long-time office mate in $\mathrm{ZH} 253$, for spreading your good mood every day and reminding me about the important things in life.

Karin Postma, I want to thank you for always being very helpful, especially when I was new in the lab, being such a nice colleague and correcting the Dutch Summary of my thesis.

I also want to acknowledge my master students, Tomasz and Marcel, for all the hard work, ideas and the great job you both did.

Thank you to all my colleagues at Targeted Therapeutics, Dwi, Deby, Iris, Acarillia, Tushar and Saleh for the good times we had together in and out of the lab and for keeping it fun. I really enjoyed the time we spend together.

Another big part of my $\mathrm{PhD}$-life were my colleagues from the other sections of the Biomaterials Science and Technology department. Chao, Jia, Ilaria, Kasia, Iris, Dooli, Denys, Natalia, Duco, Tony, Pia, Rachel, Gijs, Thijs, Odyl, Aysun, Mike, Bas. Thanks for the coffee breaks, cakes, BBQs, Borrels, Beachvolleyball matches, and Partys we had together. It was always great fun and I will miss it.

Thank you to Karin Roelofs-Haarhuis and Dennis from 20MedTherapeutics for your help with the Flow Cytometer and the NanoAssemblr, the nice chats we had and your collegiality.

The technical support and advice of Hetty, Betty, Lydia, Zlata, Kirsten, Yvonne and Marc also played an important role in the process of my PhD. I want to thank you for being so helpful and saving our labs from chaos. Karin Hendriks, you were always available for administrative questions and such a great help in finalizing the paperwork for my thesis. Thank you all! 
Bei meinen Freunden, Familie Peters und meiner eigenen Familie möchte ich mich für die Unterstützung und all die guten Zeiten bedanken. Ihr seid mir alle sehr wichtig!

Oma Ursel und Christel, Opa Arthur und Hans, Ihr wart schon ganz früh immer für mich da und ich habe für mein Leben viel von euch mitgenommen. Danke! Amelie und David, Ihr seid die besten und ich bin so froh euch zu haben! Einen riesigen Dank an meine Eltern Klaus und Bettina, für die lebenslange Unterstützung bei der Ihr mir so oft in den Hintern treten musstet! Ich bin froh mit euch über Alles reden zu können, immer guten Rat zu bekommen und so viele unterschiedliche Dinge von euch gelernt zu haben. Meine Besuche bei euch allen in Hilden füllen meinen Tank immer wieder auf!

Den größten Dank an Clara, du hast mich in den letzten Jahren am meisten unterstützt, hast mir zugehört, mich aufgebaut, mir ab und zu einen Schubser in die richtige Richtung gegeben und wir haben so viele schöne und spaßige Dinge miteinander erlebt. Ich freue mich auf mehr! Danke, danke, danke... 
